\title{
Floods on Small Streams in Texas
}

By Frederick H. Ruggles, Jr.

GEOLOGICAL SURVEY - WATER RESOURCES DIVISION Texas District

Trigg Twichell, District Chief
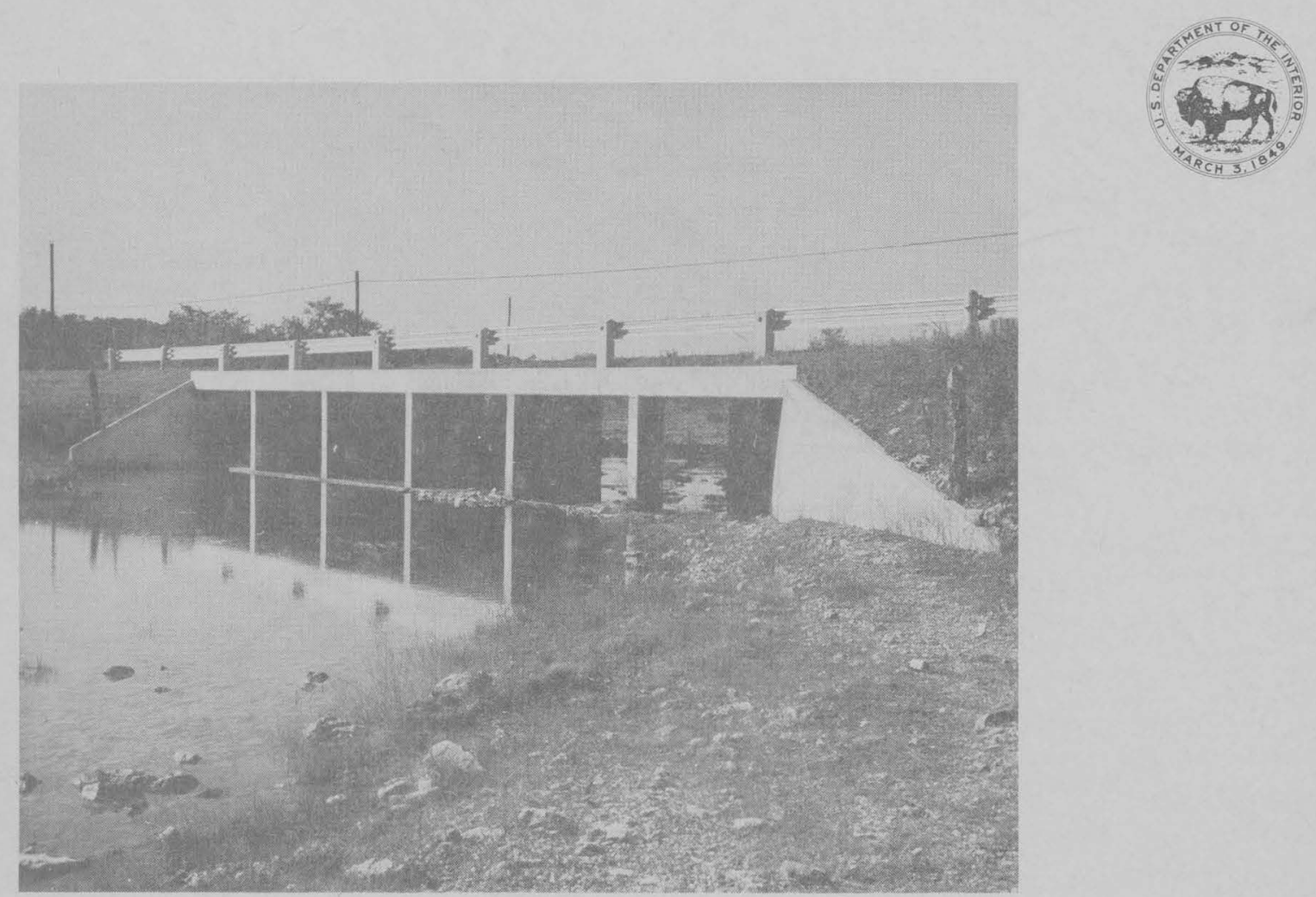

Prepared in cooperation with the Texas Highway Department and U. S. Department of Commerce, Bureau of Public Roads

Open File No. 89-January 1966 
FLOODS ON SMALL STREAMS IN TEXAS.

\begin{tabular}{c} 
RECEIVED \\
USGS WRD \\
MAY 311966 \\
ALBUQ., N. MEX. \\
\hline
\end{tabular}

By Frederick H. Ruggles, 'Jr. Hydraulic Engineer

\section{A PROGRESS REPORT}

Prepared in cooperation with the Texas Highway Department and

U. S. Department of Commerce, Bureau of Public Roads

By U. S. Geological Survey, Water Resources Division

Trigg Twichell, District Chief Austin, Texas

$$
\text { Open File } 89 \text { - January } 1966
$$


Introduction-1.-10

Purpose and scope-1..

Cooperation and acknowledgments-

Description of runoff data-..-

Definition of terms and abbreviations-

Downstream order and station numbers

Explanation of data-

Rainfall da.ta-1..

Ra,infall frequency-1.

Flood frequency-1-0.-

Selected references-.........

Station data (stations are listed after table)

Pa.ge

1

\section{ILLUSTRATIONS}

[All plates are in back of report]

Plate 1. Map showing gaging stations on small streams in Texas, 1963.

2. Map of Texas showing location of sites on small streams (other than gaging stations) where peak discharge was determined.

Figure 1. Maximum 30-minute rainfall, 10-year frequency-......2. Maximum 30-minute rainfa.11, 50-year frequency-......-

3. Maximum 1-hour rainfall, 10-year frequency-........

4. Maximum 1-hour rainfa.1l, 50-yea.r frequency-........

5. Maximum 2-hour rainfall, 10-year frequency-.......

6. Maximum 2-hour rainfall, 50-year frequency-.........

7. Maximum 3-hour rainfa.1, 10-year frequency-.........

8. Maximum 3-hour rainfall, 50-year frequency-.........

9. Maximum 6-hour rainfa.11, 10-year frequency-........-

10. Maximum 6-hour rainfall, 50-year frequency-.........-

11. Maximum 12-hour rainfall, 10-year frequency-.......-

12. Maximum 12-hour rainfa.11, 50-year frequency-.......-

13. Probable maximum 6-hour precipitation (inches) for

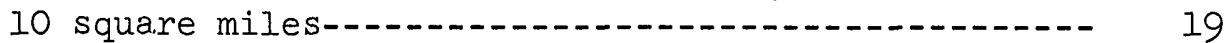

14. Area-depth rainfall curves-....... 20

11

12

13

14.

15

16

17

18

Cover Photograph

Culvert on U. S. Highway 290 west of Austin. Openings designed from flood data on small streams. (Photograph furnished by Texa.s Highway Department.) 
CONTENTS

TABLES

LIST OF GAGING STATIONS

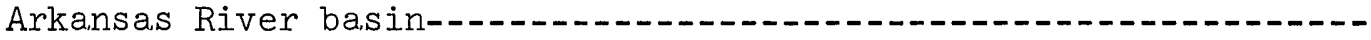
Unnamed tributary (watershed $\mathrm{W}-1$ ) of Middle Alamosa Creek near Vega, Tex. (4) 27

Unnamed tributary (watershed $\mathrm{W}-2$ ) of Middle Alamosa Creek near Vega, Tex. (4) 27

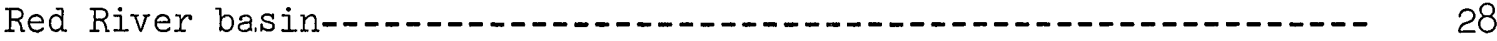

North Tule Draw at reservoir, near Tulia, Tex. (7) 28

Quitaque Creek near Quitaque, Tex. (25) 28

Bois d'Arc Creek near Randolph, Tex. (1)

Pecan Bayou near Clarksville, Tex. (1) 29

Boggy Creek near Daingerfield, Tex. (19) 30

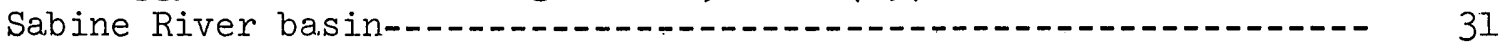

Cowleech Fork Sabine River at Greenville, Tex. (1)-....- 31

South Fork Sabine River near Quinlan, Tex. (1)

Soca.gee Creek near Carthage, Tex. (19) 32

Tenaha. Creek near Shelbyville, Tex. (Il) 33

Cypress Creek near Buna, Tex. (20) 34

Cow Bayou near Mauriceville, Tex. (20) 34

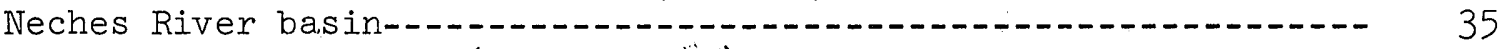

Unnamed tributary (watershed;3) of Prairie Creek near

Tyler, Tex. (10) 35

Unnamed tributary (watershed 4) of Prairie Creek near

Tyler, Tex. (10) 35

Unnamed tributary (watershed 5) of Prairie Creek near

Tyler, Tex. (10) 35

Piney Creek near Groveton, Tex. (II) 36

Arenoso Creek near San Augustine, Tex. (II)

Ayish Bayou near San Augustine, Tex. (II)

Trinity River basin-1...- 37

North Creek near Jacksboro, Tex. (2) 37

Marine Creek at Fort. Worth, Tex. (2) 37

Big Fossil Creek at Haltom City, Tex. (2) 38

Walnut Creek near Mansfield, Tex. (2) 38

Elm Fork Trinity River subwatershed 6-0 near Muenster, Tex.

(3) 


\section{CONTENTS}

Trinity River basin--Continued

Elm Fork Trinity River near Muenster, Tex. (3) Little Elm Creek near Aubrey, Tex. (18) Turtle Creek at Da.llas, Tex. (18) White Rock. Creek a.t Keller Springs Rd., Dallas, Tex. (18)---Spanky Branch at McCallum Lane, Dallas, Tex. (18)-..Cottonwood Creek at Forest Lane, Dallas, Tex. (18) Floyd Branch at Forest Lane, Dallas, Tex. (18) White Rock Creek a.t Greenville Ave., Dallas, Tex. (18)-..--Ash Creek at Highland Road, Da.lla.s, Tex. (18) Forney Creek at Lawnview Ave., Dallas, Tex. (18) Honey Creek subwatershed No. Il near McKinney, Tex. (18)-.-.Honey Creek subwatershed No. 12 near McKinney, Tex. (18)-.-.Honey Creek near McKinney, Tex. (18) Unnamed tributary (watershed WI) of Duck Creek near Garland,

Tex. (18)- 46

Unnamed tributary (watershed WIII) of Duck Creek near

Garland, Tex. (18) 46

Unnamed tributary (watershed WIV) of Duck Creek near

Garland, Tex. (18)

Duck Creek near Garland, Tex. (18)

Pin Oak Creek near Hubbard, Tex. (9)

San Jacinto River basin-.......

Whiteoak Bayou a.t Houston, Tex. (12) Brays Bayou at Houston, Tex. (12) Sims Bayou at Houston, Tex. (12) Greens Bayou near Houston, Tex. (12) Ha.lIs Bayou at Houston, Tex. (12)

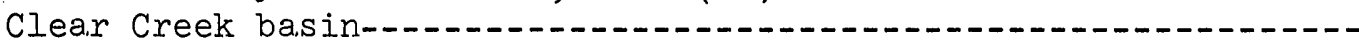

Clear Creek near Pearland, Tex. (12)

Chocolate Bayou basin-....... 53

Chocolate Bayou near Alvin, Tex. (12) 53

Brazos River basin-..... 54

Unnamed tributary (watershed 1) of Duck Creek near Spur, Tex.

(25)

Unnamed tributary (watershed 2) of Duck Creek near Spur, Tex. (25) -

Unnamed tributary (watershed 3) of Duck Creek near Spur, Tex.

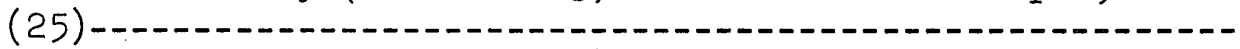

Unnamed tributary (watershed 5) of Duck Creek near Spur, Tex.

(25) -

Unnamed tributary (watershed 6) of Duck Creek near Spur, Tex.

(25) -

Unnamed tributary (watershed 1l) of Duck Creek near Spur,

Tex. (25)

Unnamed tributary (wa.tershed 12) of Duck Creek nea.r Spur,

Tex. (25) 
Brazos River basin--Continued

Unnamed tributary (watershed 14) of Duck Creek near Spur,

Tex. (25) 56

Unnamed tributary (watershed 15) of Duck Creek near Spur,

Tex. (25)- 56

Salt Croton Creek near Aspermont, Tex. (8) 57

North Fork Hubbard Creek near Albany, Tex. (8)

Salt Creek at Olney, Tex. (3) 58

Salt Creek near Newcastle, Tex. (3) 58

Oak Creek near Graham, Tex. (3)

North Bosque River at. Stephenville, Tex. (2) 59

Green Creek subwatershed No. I near Dublin, Tex. (2) -...... 60

Green Creek near Alexander, Tex. (2)

Hog Creek near Crawford, Tex. (9) 61

Sandy Creek watershed SW-16 near Riesel, Tex. (9)-..... 61

Cow Bayou subwatershed No. 4. near Bruceville, Tex. (9)-...-. 62

Cow Bayou at Mooreville, Tex. (9) 62

Deer Creek at Chilton, Tex. (9)

Brushy Creek watershed A near Riesel, Tex. (9)-......-

Brushy Creek watershed C near Riesel, Tex. (9)........

Brushy Creek watershed D near Riesel, Tex. (9)

Brushy Creek watershed SW-14 near Riesel, Tex. (9)-.....-

Brushy Creek. watershed SW-12 near Riesel, Tex. (9)

Brushy Creek watershed Y-10 near.Riesel, Tex. (9)-.....-

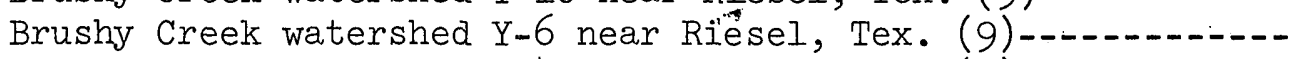

Brushy Creek watershed Y-4 near Riesel, Tex. (9)

Brushy Creek watershed Y-8 hear Riesel, Tex. (9)-.....

Brushy Creek watershed Y-2 near Riesel, Tex. (9)

Brushy Creek watershed Y-7 near Riesel, Tex. (9)-.....-

Brushy Creek watershed SW-7 near Riesel, Tex. (9)-.......-

Brushy Creek watershed SW-13 near Riesel, Tex. (9)

Brushy Creek watershed Y near Riesel, Tex. (9)

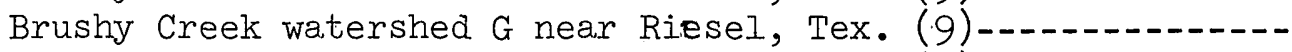

Brushy Creek watershed $\mathrm{Z}$ near Riesel, Tex. (9)

Brushy Creek"watershed V near Riesel, Tex. (9)

Brushy Creek watershed SW-18 near Riesel, Tex. (9)-.....-

Brushy Creek watershed SW-1l near Riesel, Tex. (9).......-

Brushy Creek watershed SW-17 near Riesel, Tex. (9)........

Brushy Creek watershed. SW-5 near Riesel, Tex. (9)

Brushy Creek watershed. W-I near Riesel, Tex. (9)-....-

Brushy Creek watershed.SW-3 near Riesel, Tex. (9).........

Brushy Creek watershed W-6 near Riesel, Tex. (9)-.......

Brushy Creek watershed: SW-2 near Riesel, Tex. (9).........

Brushy Creek watershed W-10 near Riesel, Tex. (9)

Brushy Creek watershed W-2 near Riesel, Tex. (9)

Brushy Creek watershed SW-6 near Riesel, Tex. (9)

Big Elm Creek near Temple, Tex. (9)

63

63

64.

64

65

65

66

66

67

67

68

68

69

69

69

70

70

71

71

71

72

72

73

73

74.

74

75

75

76

76 
Brazos River basin--Continued

North Elm Creek near Ben Arnold, Tex. (17)

Big Creek near Needville, Tex. (12)

Fairchild Creek near Needville, Tex. (12)

Dry Creek near Rosenberg, Tex. (12)

Dry Creek near Richmond, Tex. (12)

Colorado River basin-_._. 79

Bluff Creek near Ira, Tex.(8)

Graze Creek near Westbrook, Tex. (8) 80

Pecan Creek near San Angelo, Tex. (7) 81

Mukewater Creek subwatershed No. 9 near Trickham, Tex. (23)-- 81

Mukewater Creek a.t Trickham, Tex. (23) 82

Deep Creek near Mercury, Tex. (23) 82

Deep Creek subwatershed No. 8 (Dry Prong Deep Creek) near

Mercury, Tex. (23)

Dry Prong Deep Creek near Mercury, Tex. (23)

Waller Creek at 38th Street, Austin, Tex. (14) Waller Creek at 23d Street, Austin, Tex. (14) Dry Creek a.t Buescher Lake, near Smithville, Tex. (14)-..-.-

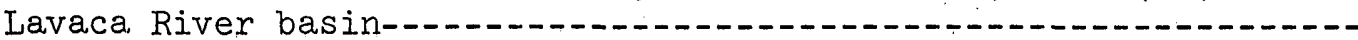

Lavaca River at Hallettsville, Tex. (13)

Guadalupe River basin-........

Johnson Creek near Ingram, Tex. (15)

Rebecca Creek near Spring Branch, Tex. (15)

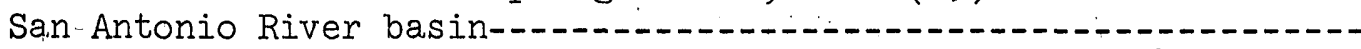

San Antonio River at San Antonio, Tex. (15)

San Pedro Creek a.t. San Antonio, Tex. (15)

Red Bluff Creek near Pipe Creek, Tex. (15)

Calaveras Creek subwatershed No. 6 near Elmendorf, Tex. (15)-

Callaveras Creek near Elmendorf, Tex. (15)

Escondido Creek subwatershed No. I near Kenedy, Tex. (16)-..-

Escondido Creek a.t Kenedy, Tex. (16)

Dry Escondido Creek near Kenedy, Tex. (16)

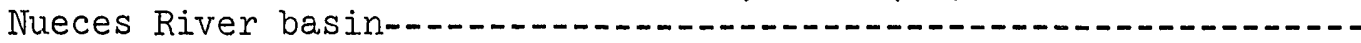

Dry Frio River near Reagan Wells, Tex. (22)

Hondo Creek near Tarpley, Tex. (15)

Seco Creek at Miller Ranch, near Utopia, Tex. (22)

Seco Creek near Utopia, Tex. (22)

Seco Creek near D'Hanis, Tex. (15)

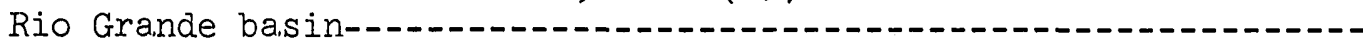

McKelligon Canyon at El Paso, Tex. (24)

Government ditch at El Paso, Tex. (24)

Madera Canyon near Toyahvale, Tex. (24)

Cienegas Creek near Del Rio, Tex. (22)

San Felipe Creek near Del Rio, Tex. 
FLOODS ON SMALL. STREAMS IN TEXAS

By Frederick H. Ruggles, Jr.

\section{INTRODUCTION}

The first streamflow station in Texas was established on the Rio Grande at El Paso on May 10, 1889. Since that time the systematic collection of streamflow data has expanded. In 1915 the Texas Board of Water Engineers (now the Texas Water Development Board) entered into a coopera.tive agreement with the U. S. Geological Survey for the purpose of expanding the network of stream-gaging stations in Texas. Sites were selected for stream-gaging stations to obtain hydrologic data for water supply and flood control. Therefore, the stream-gaging stations were located principally on major streams. Today, after three-quarters of a century of hydrologic data collection, peak discharge data on small streams are still deficient in Texas. The Geological Survey and the Texas Highway Department, therefore, have entered into a cooperative program to collect peak discharge data on small streams for the purpose of deriving flood-frequency data needed for the economical design of culverts and small bridges.

\section{Purpose and Scope}

This report has been prepared to combine into one volume, the available runoff and rainfall information that might be used in the design of drainage structures spanning small streams in Texas. The report and information presented are considered interim to the later presentation of more useable flood data being obtained from a recently initiated comprehensive data-collection program on streams with drainage areas ranging from 1 to 20 square miles. A summary of flood data for stream-gaging stations and partial-record stations, a list of peak discharges for floods at miscellaneous sites on small streams, a summary of outstanding point rainfall amounts, a brief rainfall-frequency presentation, and a. flood-frequency discussion are given in this report.

Data presented herein supplement that given in a report by Patterson (1963). Patterson's report presented flood-peak data and methods for predicting magnitude and frequency of floods on most streams in Texas. The available data necessarily limited Patterson's analyses to those involving streams with drainage areas in excess of 100 square miles. 


\section{Cooperation and Acknowledgments}

The report was prepared under provisions of a cooperative agreement between the Texa.s Highway Department and the Geölogical Survey. The assistance of the Bureau of Public Roads, U.S. Department of Commerce, is gratefully acknowledged.

Trigg Twichell, district chief, Geological Survey, Austin, Texas, directed the preparation of this report.

\section{DESCRIPTION OF RUNOFF DATA}

\section{Definition of Terms and Abbreviations}

The terms of streamflow and other hydrologic data, a.s used in this report, are defined as follows:

Stream-gaging station is a particular site on a stream or a canal where systematic observations of gage height or discharge are obtained on a continuous basis.

Partial-record station is a particular site where limited streamflow data (usually either low flow or high flow) are collected systematically over a period of years for use in hydrologic analyses.

Cubic foot per second (cfs) is the rate of discharge of a stream whose channel is I square foot in cross-sectional area and whose average velocity is 1 foot per second.

Cubic feet per second per square mile (cfsm) is the average number of cubic feet of water flowing per second from each square mile of area. drained, assuming that the runoff is distributed uniformly in time and area.

Stage-discharge relation is the relation between water surface elevation (gage height) and the amount of water flowing in a channel, expressed as volume per unit of time.

The dra,inage area of a stream at a specified location is that area, measured in a horizontal plane, which is so enclosed by a topographic divide that direct surface runoff from precipitation normally would drain by gravity into the river above the specified point. Figures of drainage a.rea given herein include all closed basins, or noncontributing areas, within the area unless otherwise noted.

Partial-duration flood series. A list of all flood peaks that exceed a chosen base stage or discharge, regardless of the number of peaks occurring in a year. (Also called basic stage flood series, or floods above a ba.se.) 
In this report, in a downstream direction along the main stem, all stations on a tributary entering above a main-stem station are listed before that station. If a tributary enters between two main-stem stations, it is listed between them.

As a means of identification, each gaging station has been assigned a station number. The numbers have been assigned in the downstream order used in this report. In assigning station numbers, no distinction is made between partial-record stations and stream-gaging stations, so that the station number for a partial-record station indicates downstream order position in a list made up of both types of stations. Gaps are left in the numbers to allow for new stations that may be esta.blished; hence, the numbers are not consecutive.

The complete number for each station, such as 08-0100.00, includes the part number " 8 " and a six-digit station number and is located to the left of the station name in "Station da.ta." In this report the part number and only the essential digits of the complete number are shown. For example, for a station with the complete number 08-0100.00, the station number shown is 8-100. The part number is a means of identifying the major drainage system in which a station is located. Part 7 is drainage into the Lower Mississippi River basin and Part 8 is arainage into the Western Gulf of Mexico. In the "Station data" section of this report a number appears to the right of all gaging station names; this number corresponds to the State Highway District in which the. station is located.

\section{Explanation of Data}

A summary of pertinent flood data through September 30, 1963, for all ga.ging stations with drainage areas of about 100 square miles or less is given in the section, "Section data." The locations of these stations are shown on plate 1. Numbers shown on the map are station identification numbers. Data summary for each station consists of a description of the station and a list of peaks for the period of record. When 25 percent or more of the total runoff at a gaging station is artifically controlled, the record for the site has not been included.

The station description gives the location of the gaging station, drainage area, type and datum of:gages, a brief statement concerning the stage-discharge relation, bankfull stage (where defined), historical flood data, and some general remarks concerning the station. Records furnished by another agency are so identified. The location and the drainage area for the gaging station are obtained from the best available maps. The datum of the gage is the elevation of the zero of the gage above mean sea level. Historical data were obtained through 
interviews with local residents and from local newspapers. These historical data are shown for information, and generally the authority for the data is given. Under "Remarks," information is given on factors that affect the peak flow, such as storage or regulation, and other pertinent factors, such as rainfall stations operated by the Geological Survey in the basin and the base for the partial-duration series.

Peak flows above a base are tabulated for most stations; where peaks above a base are not available, only annual peaks are shown. The data have been tabulated on a water-year basis unless otherwise noted. The water year begins October 1 and ends September 30 and is identified by the year in which it ends; thus, a peak that occurred in october, November, or December 1950 would be listed in the 1951 water year.

Table 1 (after References) is a list of peak discharges for floods at miscellaneous sites. These data have been gathered over the years because of the outstanding nature of the event, or for a specific design problem. Headings and data are self-explanatory. These sites are shown on plate 2. May numbers refer to identification numbers shown in column 1 of table 1 .

\section{RAINFALI DATA}

Climatic conditions in Texas range from humid along the lower reaches of the Sabine River to arid in the extreme western part of the State. Mean annual precipitation is 55 inches on the southeast boundary of Texas and decreases fairly uniformly westward to less than 10 inches near El Paso. Rainfall is erratic with long periods of little or no precipitation, followed at times by high intensity rainfalls of unusual quantities. Some of the highest rates of rainfall recorded in the United States have occurred in Texas. At D'Hanis in Medina County, 21.5 inches of rain fell: in 3 hours on May 31, 1935, and at Thrall in Williamson County, 38.2 inches fell in 24 hours on September 9 and 10, 1921. A 20-inch rainfall in 24 hours has been recorded in most parts of the state.

Floods in Texas are caused by several different types of storms and may occur during any month of the year. Great floods have occurred most frequently during the months from April to October. Most of the major floods are caused by tropical or semi-tropical storms from the Gulf of Mexico. Some floods, such as those of April through June 1957, are caused by cooler air from the northwest colliding with warm, moisture-laden air from the Gulf. On rare occasions, large floods have been caused by storms crossing Mexico and the United States from the Pacific Ocean. Floods in the western part of the state are frequently caused by thunderstorms of high intensity which usually cover relatively small areas and occur during the summer or early fall. Following is a list of some outstginding point rainfalls in Texas that have been documented through the years. 


\begin{tabular}{|c|c|c|c|c|c|}
\hline Site & & Date & $\begin{array}{r}\text { Duration } \\
\text { (time) }\end{array}$ & $\begin{array}{c}\text { Amount } \\
\text { (inches) }\end{array}$ & Authority \\
\hline $\begin{array}{l}\text { Thrall } \\
\text { Hearne } \\
\text { Taylor } \\
\text { San Antonio } \\
\text { Austin } \\
\text { Carlsbad } \\
\text { Moulton } \\
\text { San Antonio (near) } \\
\text { Westfield }\end{array}$ & $\begin{array}{l}\text { Sept. } \\
\text { June } \\
\text { Sept. } \\
\text { Sept. } \\
\text { Sept. } \\
\text { Sept. } \\
\text { June } \\
\text { Sept. } \\
\text { Dec. }\end{array}$ & $\begin{array}{r}9-10,1921 \\
28,1899 \\
9,1921 \\
9-10,1946 \\
9,1921 \\
16,1936 \\
30,1940 \\
9,1921 \\
7,1935\end{array}$ & $\begin{array}{l}24 \mathrm{~h} \\
24 \mathrm{~h} \\
24 \mathrm{~h} \\
24 \mathrm{~h} \\
24 \mathrm{~h} \\
24 \mathrm{~h} \\
24 \mathrm{~h} \\
24 \mathrm{~h} \\
24 \mathrm{~h}\end{array}$ & $\begin{array}{l}38.2 \\
24.0 \\
23.11 \\
21.0 \\
19.03 \\
18.0 \\
17.98 \\
17.0 \\
15.48\end{array}$ & $\begin{array}{l}\text { U. S. Weather Bureau } \\
\text { U. S. Weather Bureau } \\
\text { U. S. Weather Bureau } \\
\text { U. S. Geological Survey } \\
\text { U. S. Weather Bureau } \\
\text { U. S. Geological Survey } \\
\text { U. S. Weather Bureau } \\
\text { U. S. Geological Survey } \\
\text { U. S. Geological Survey }\end{array}$ \\
\hline Smithville & June & 30,1940 & $15 \mathrm{~h}$ & 16.0 & U. S. Weather Bureau \\
\hline $\begin{array}{l}\text { Thrall } \\
\text { Taylor } \\
\text { Engle }\end{array}$ & $\begin{array}{l}\text { Sept. } \\
\text { Sept. } \\
\text { June }\end{array}$ & $\begin{array}{rr}9-10, & 1921 \\
9, & 1921 \\
30, & 1940\end{array}$ & $\begin{array}{ll}12 & h \\
12 & h \\
12 & h\end{array}$ & $\begin{array}{l}29.8 \\
18.96 \\
17.5\end{array}$ & $\begin{array}{l}\text { U. S. Weather Bureau } \\
\text { U. S. Weather Bureau } \\
\text { U. S. Weather Bureau }\end{array}$ \\
\hline San Antonio ( $11 \mathrm{mi} \mathrm{SE}$ ) & Sept. & 27,1946 & $11 \mathrm{~h}$ & 16.67 & U. S. Geological Survey \\
\hline $\begin{array}{l}\text { De Leon }(5.5 \mathrm{mi} \mathrm{E}) \\
\text { Fort Worth }\end{array}$ & $\begin{array}{l}\text { May } \\
\text { May }\end{array}$ & $\begin{array}{rr}23, & 1952 \\
16-17, & 1949\end{array}$ & $\begin{array}{l}9 \mathrm{~h} \\
9 \mathrm{~h}\end{array}$ & $\begin{array}{l}20.0 \\
11.0\end{array}$ & $\begin{array}{l}\text { U. S. Geological Survey } \\
\text { U. S. Geological Survey }\end{array}$ \\
\hline Frisco & Sept. & 21,1964 & $8 \mathrm{~h}$ & 14.0 & U. S. Geological Survey \\
\hline $\begin{array}{l}\text { Thrall } \\
\text { Adell } \\
\text { Taylor } \\
\text { Mineral Wells }\end{array}$ & $\begin{array}{l}\text { Sept. } \\
\text { July } \\
\text { Sept. } \\
\text { July }\end{array}$ & $\begin{array}{r}9-10,1921 \\
27,1962 \\
9,1921 \\
27,1962\end{array}$ & $\begin{array}{l}6 \mathrm{~h} \\
6 \mathrm{~h} \\
6 \mathrm{~h} \\
6 \mathrm{~h}\end{array}$ & $\begin{array}{l}19.6 \\
17 \\
14.16 \\
14.0\end{array}$ & $\begin{array}{l}\text { U. S. Weather Bureau } \\
\text { Texas Water Commission } \\
\text { U. S. Weather Bureau } \\
\text { Texas Water Commission }\end{array}$ \\
\hline Fort Worth & Sept. & 7,1962 & $5 \mathrm{~h}$ & 11.5 & U. S. Geological Survey \\
\hline $\begin{array}{l}\text { D'Hanis } \\
\text { Taylor } \\
\text { Dallas }\end{array}$ & $\begin{array}{l}\text { May } \\
\text { Sept. } \\
\text { Oct. }\end{array}$ & $\begin{array}{r}31,1935 \\
9,1921 \\
8,1962\end{array}$ & $\begin{array}{ll}3 h \\
3 h \\
3 h\end{array}$ & $\begin{array}{l}21.5 \\
10.72 \\
7.46\end{array}$ & $\begin{array}{l}\text { U. S. Weather Bureau } \\
\text { U. S. Weather Bureau } \\
\text { U. S. Geological Survey }\end{array}$ \\
\hline Lelia. Lake & June & 15,1938 & $2 \frac{1}{2} h$ & 14 & U. S. Geological Survey \\
\hline $\begin{array}{l}\text { Galveston } \\
\text { Taylor }\end{array}$ & $\begin{array}{l}\text { Apr. } \\
\text { Sept. }\end{array}$ & $\begin{aligned} 22, & 1904 \\
9, & 1921\end{aligned}$ & $\begin{array}{ll}2 & h \\
2 & h\end{array}$ & $\begin{array}{l}7 \cdot 58 \\
7 \cdot 51\end{array}$ & $\begin{array}{l}\text { U. S. Weather Bureau } \\
\text { U. S. Weather Bureau }\end{array}$ \\
\hline $\begin{array}{l}\text { Galveston } \\
\text { Del Rio } \\
\text { El Paso } \\
\text { Taylor } \\
\text { Dallas } \\
\text { Abilene } \\
\text { Amarillo }\end{array}$ & $\begin{array}{l}\text { Oct. } \\
\text { Feb. } \\
\text { July } \\
\text { Sept. } \\
\text { Oct. } \\
\text { July } \\
\text { June }\end{array}$ & $\begin{array}{r}22,1913 \\
27,1921 \\
9,1881 \\
9,1921 \\
8,1962 \\
31,1911 \\
24,1948\end{array}$ & $\begin{array}{ll}l & h \\
l & h \\
l & h \\
l & h \\
l & h \\
l & h \\
l & h\end{array}$ & $\begin{array}{l}5.31 \\
4.82 \\
4.80 \\
4.25 \\
3.95 \\
3.47 \\
3.36\end{array}$ & $\begin{array}{l}\text { U. S. Weather Bureau } \\
\text { U. S. Weather Bureau } \\
\text { U. S. Weather Bureau } \\
\text { U. S. Weather Bureau } \\
\text { U. S. Geological Survey } \\
\text { U. S. Weather Bureau } \\
\text { U. S. Weather Bureau }\end{array}$ \\
\hline $\begin{array}{l}\text { El Paso } \\
\text { Taylor }\end{array}$ & $\begin{array}{l}\text { July } \\
\text { Sept. }\end{array}$ & $\begin{array}{l}9,1881 \\
9,1921\end{array}$ & $\begin{array}{l}30 \mathrm{~m} \\
30 \mathrm{~m}\end{array}$ & $\begin{array}{l}3.09 \\
2.89\end{array}$ & $\begin{array}{l}\text { U. S. Weather Bureau } \\
\text { U. S. Weather Bureau }\end{array}$ \\
\hline Taylor & Sept. & 9,1921 & $15 \mathrm{~m}$ & 2.53 & U. S. Weather Bureau \\
\hline Galveston & June & 4,1871 & $14 \mathrm{~m}$ & 3.95 & U. S. Weather Bureau \\
\hline Taylor & Sept. & 9,1921 & $10 \mathrm{~m}$ & 2.00 & U. S. Weather Bureau \\
\hline Taylor & Sept. & 9,1921 & $5 \mathrm{~m}$ & 1.30 & U. S. Weather Bureau \\
\hline
\end{tabular}

a) h - hour

m - minute 


\section{RAINFALL FREQUENCY}

For the conterminous United States the U. S. Weather Bureau has published a report (Hershfield, 1961) that is a convenient summary of empirical relationships, working guides, and maps useful in practical problems requiring rainfall frequency data. The paper is divided into two parts. The first part presents the rainfall analyses. The second part presents rainfall frequency maps based on a comprehensive collection of up-to-date statistics, several related maps, and seasonal variation diagrams. The rainfall frequency maps are for selected durations from 30 minutes to 24 hours and return periods from 1 to 100 years. Figures 1-12 are maps of Texas, showing the maximum 30-minute, 1-, 2-, 3-, 6-, and 12-hour rainfall for 10- and 50-year frequencies. Figure 13 is a map showing the probable maximum 6-hour precipitation for a 10-square mile area.

For larger drainage areas, consideration must be given not only to point rainfall, but to average depth over the entire basin. The areadepth curves shown in figure 14 can be used with data obtainable from figures 1-12 to estimate the probable areal extent of rainfalls of varying magnitude, duration, and frequency. The method is to choose a point rainfall value from the appropriate map of figures $1-12$ and multiply it by the appropriate value selected from figure 14. The resulting product is the average depth of rainfall over that area for the chosen duration and frequency.

In the absence of factual runoff-frequency data, the preceding rainfallfrequency data can be used to estimate runoff of specific frequency for the design of storm drainage structures. However, the possible inaccuracies in the runoff values estimated in this manner should be recognized. Obviously, a storm rainfall of 10-year frequency will not always result in a peak discharge of 10-year frequency; therefore, hydrologists always prefer to use basic runoff da.ta when available. Da.ta on rainfall magnitude and frequency are presented here solely to provide the reader with all the available data, both runoff and rainfall, usable in the design of drainage structures on small streams in Texas.

\section{FLOOD FREQUENCY}

In recent years the Geological Survey has prepared two publications (Patterson, 1963; Benson, 1964) dealing with flood frequency in Texas. Patterson outlines methods by which the magnitude and frequency of expected floods for most large streams in Texas can be predicted. Methods outlined by Patterson will be the basis for flood design used in the state until more data make possible additional flood-frequency analyses. 


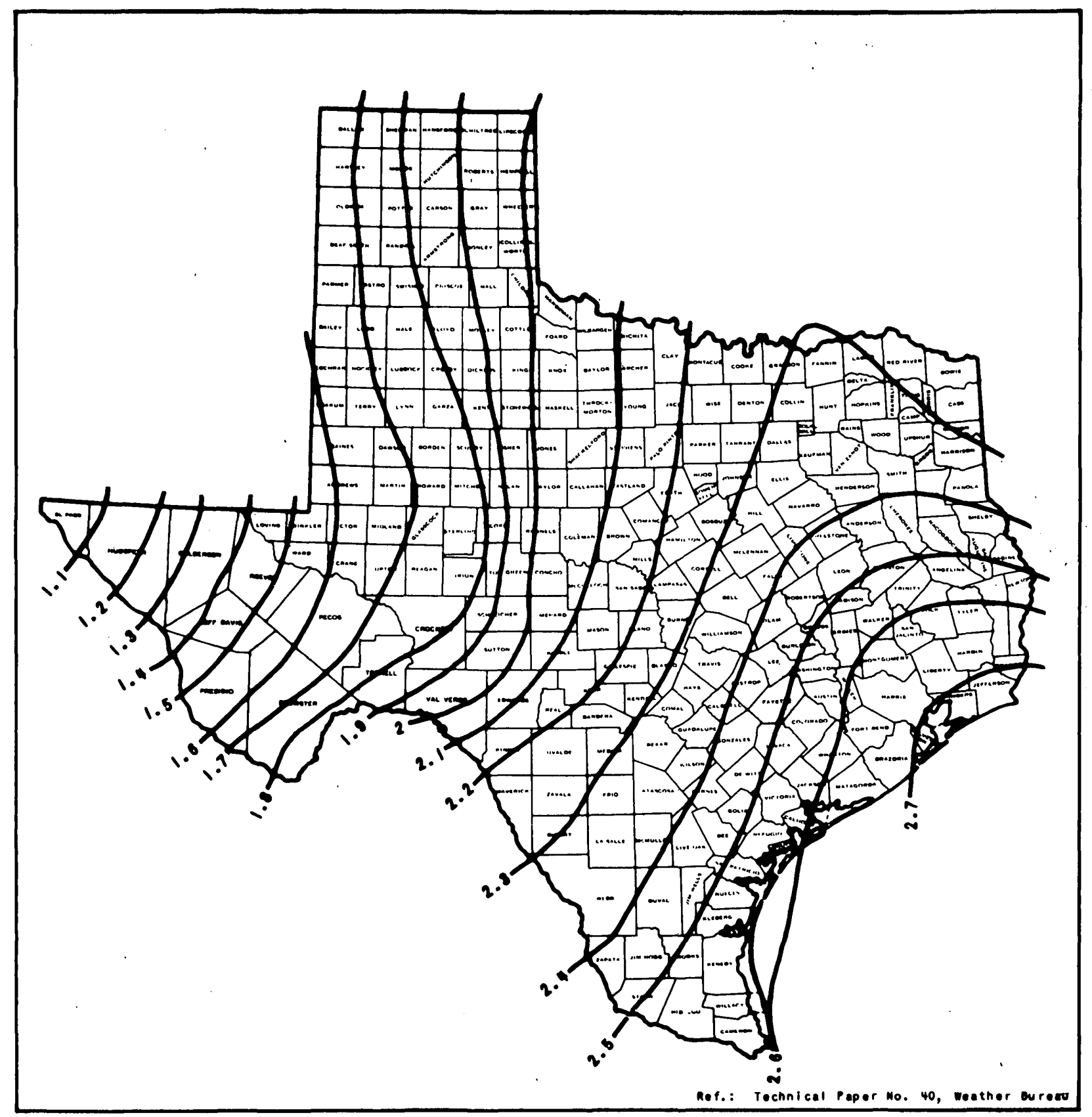

Flgure 1. - Meximum 30-minute rainfoll, ten-year frequency in Texas 


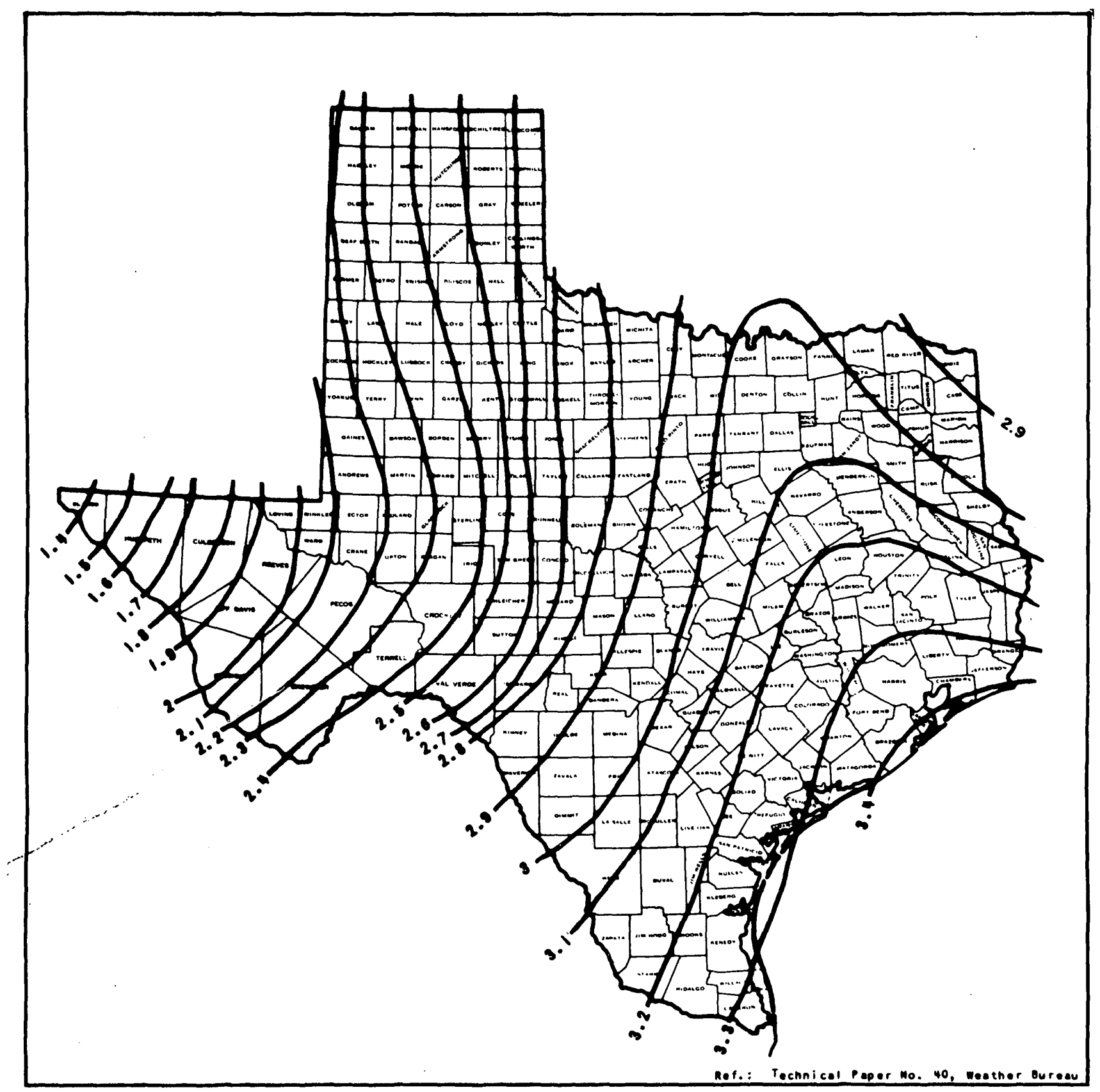

Flgure 2. Maximum 30-minute rainfall, 50-year frequency in Texos 


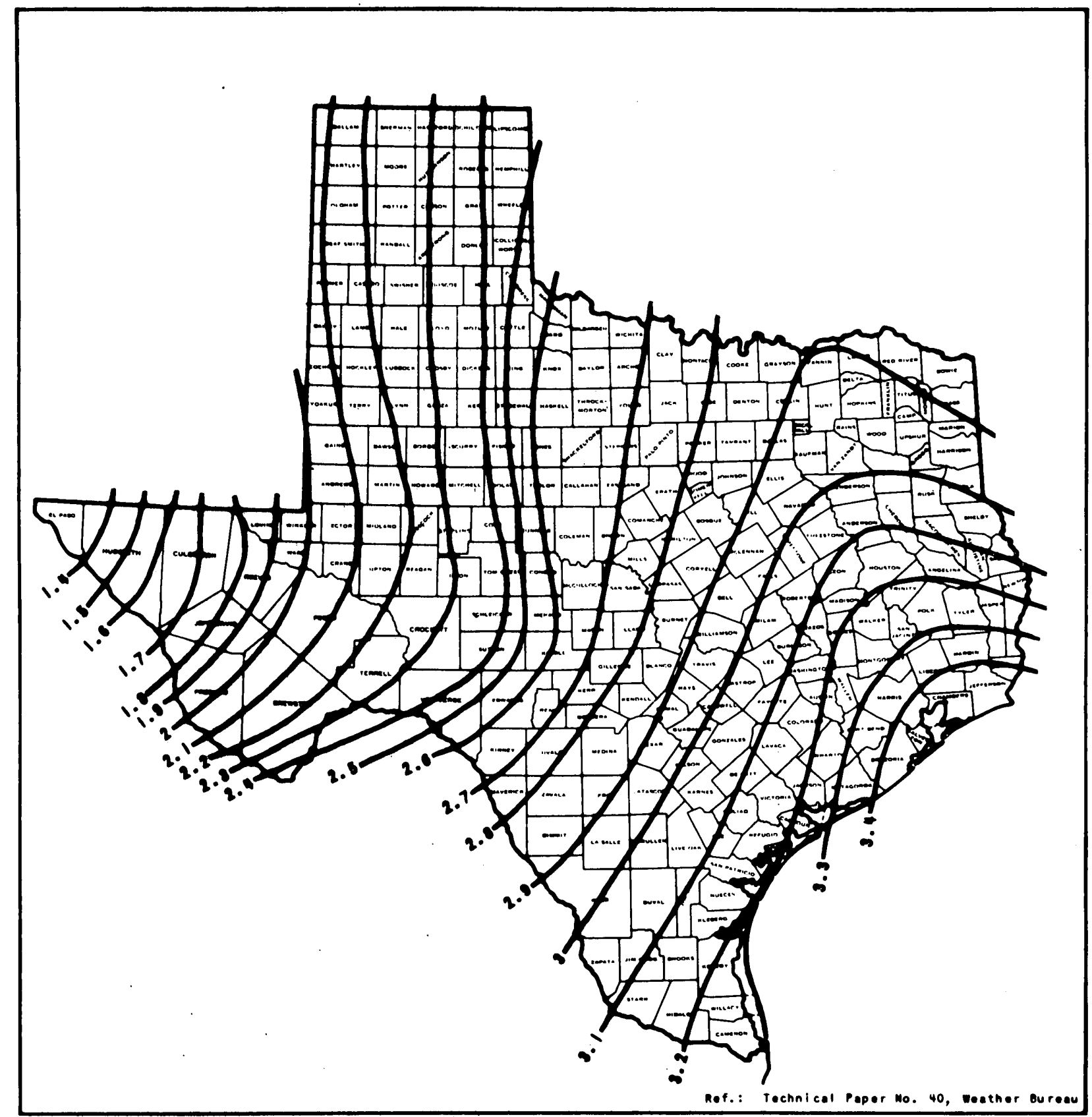

Figure 3.-Maximum one-hour rainfali, 10-year frequency. in Texas 


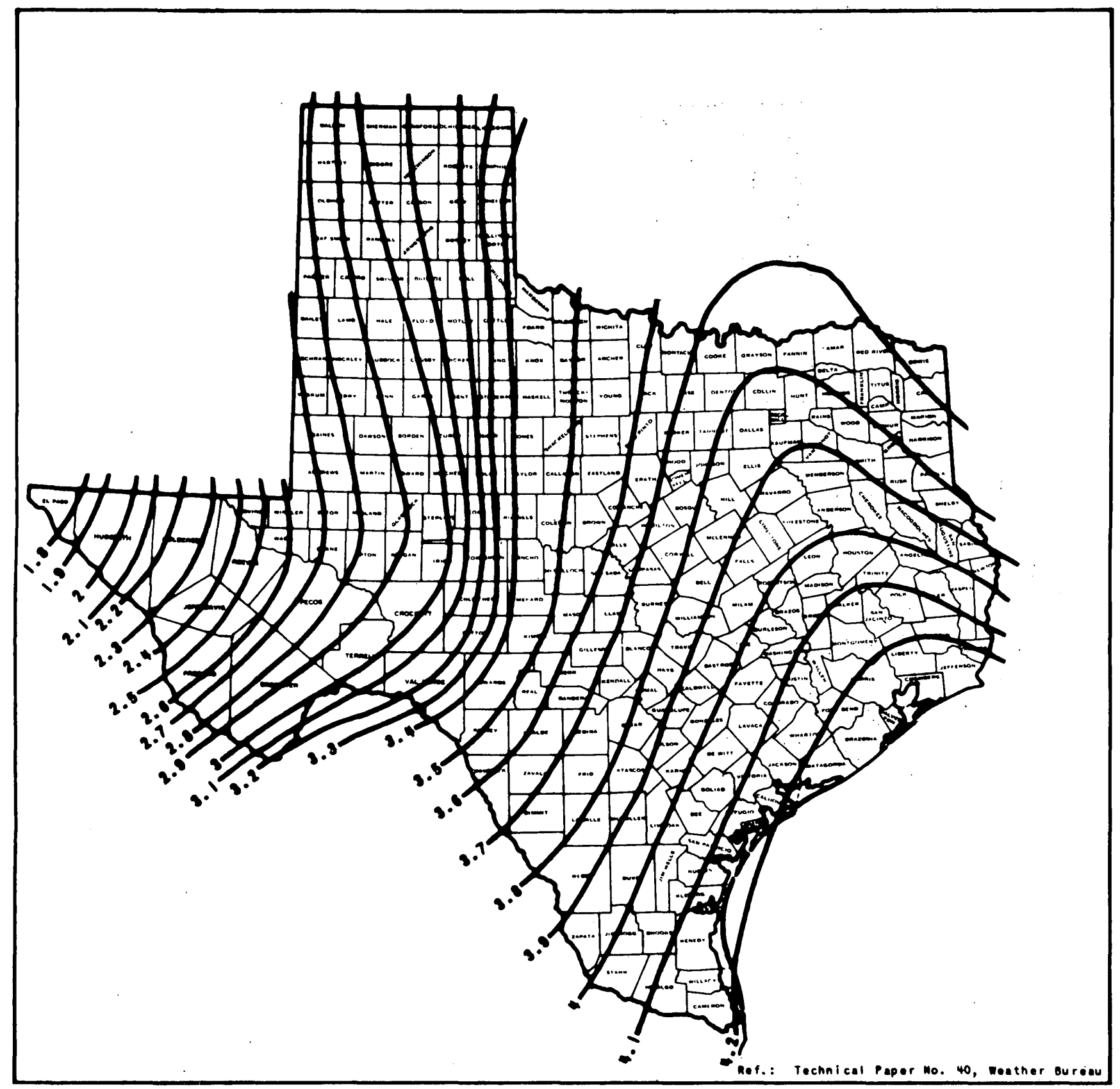

Figure 4.-Moximum one-hour roinfoll, 50-yeor frequency in Texas 


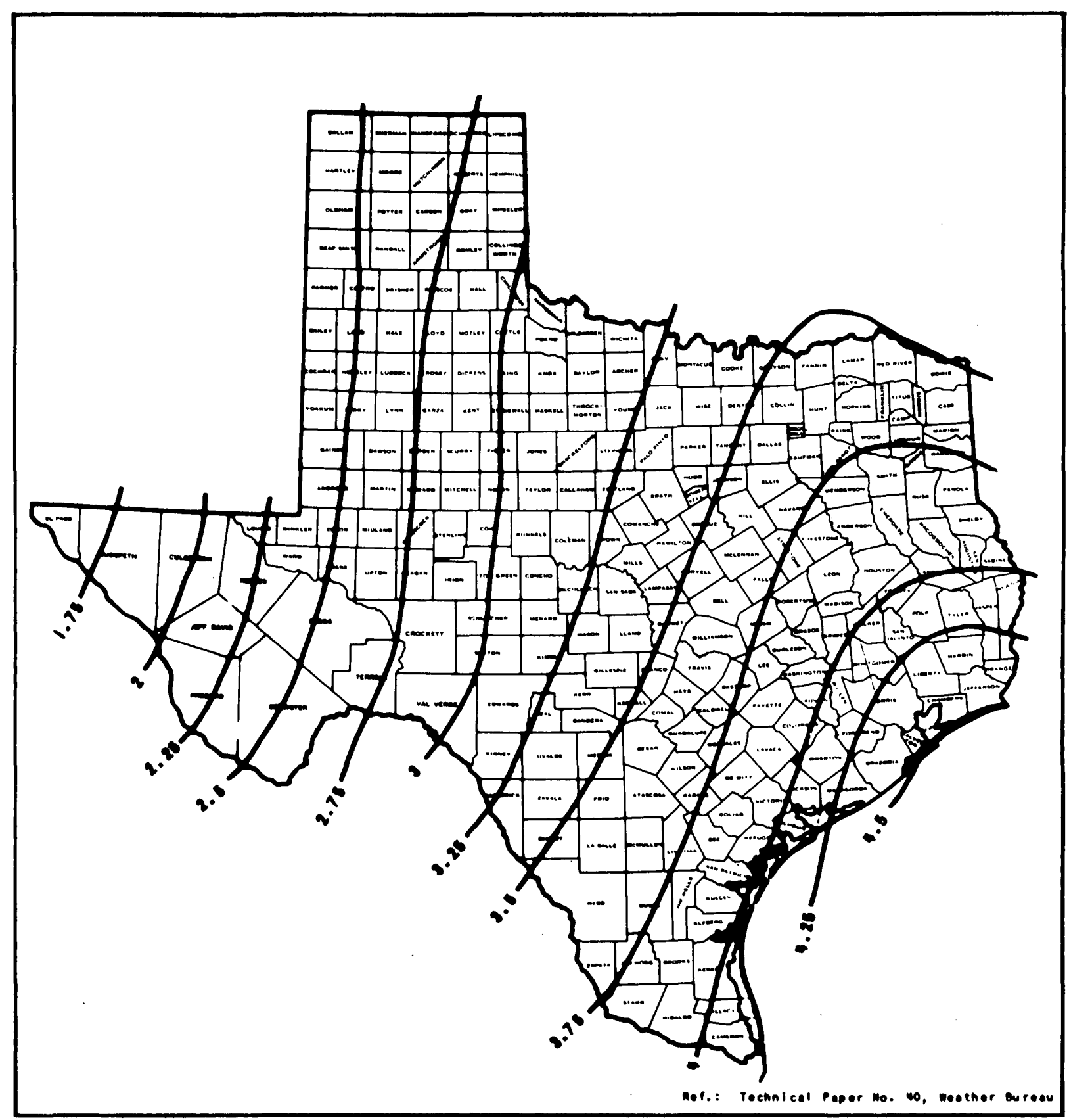

Flgure 5.-Moximum two-hour roinfall, 10-yeor frequency in Texas 


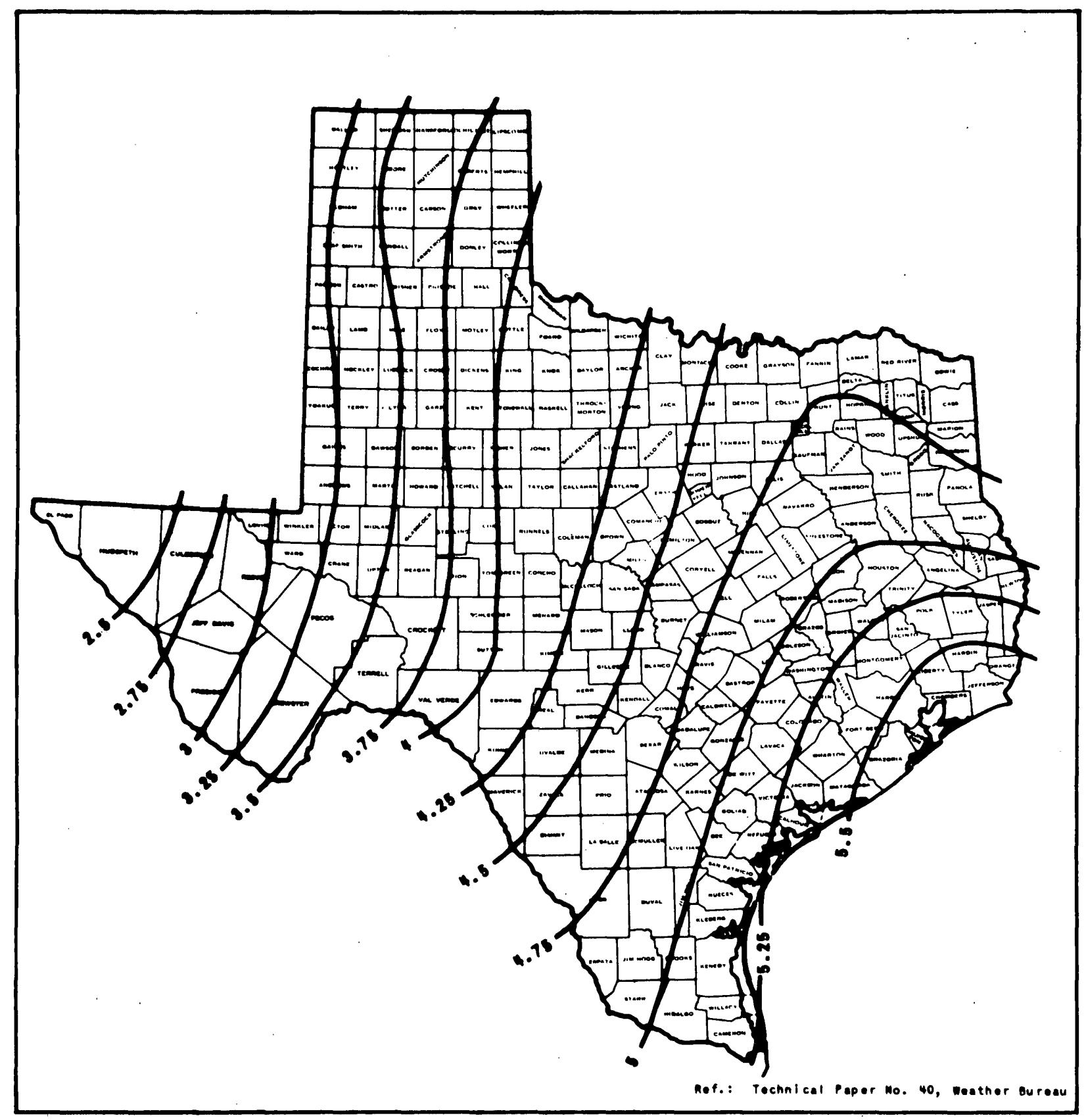

Flgure 6. -Maximum two-hour rainfoll, 50-yeor frequency in Texos 


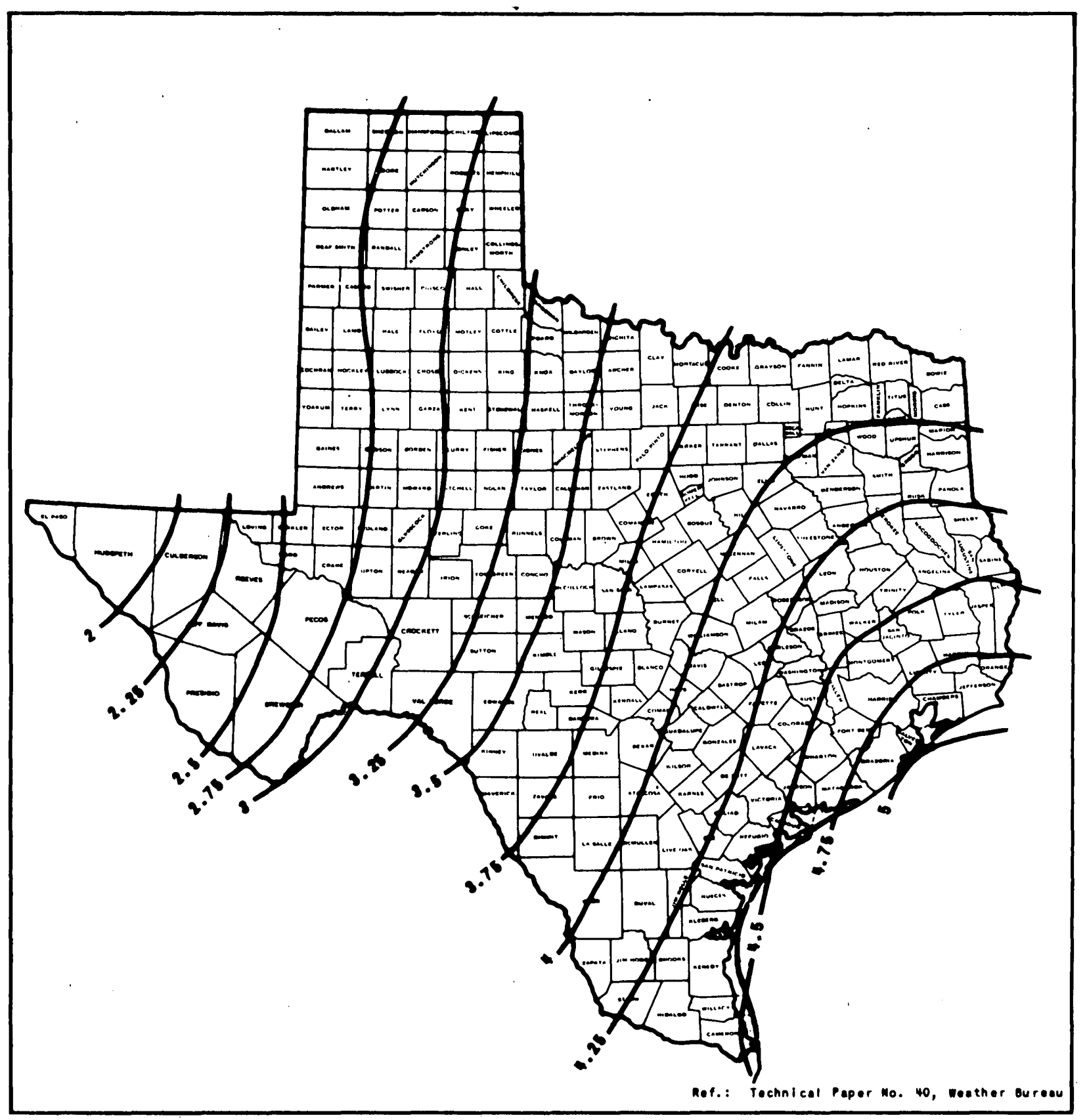

Figure 7.-Meximum three-hour ralnfall, 10-year frequency in Texas 


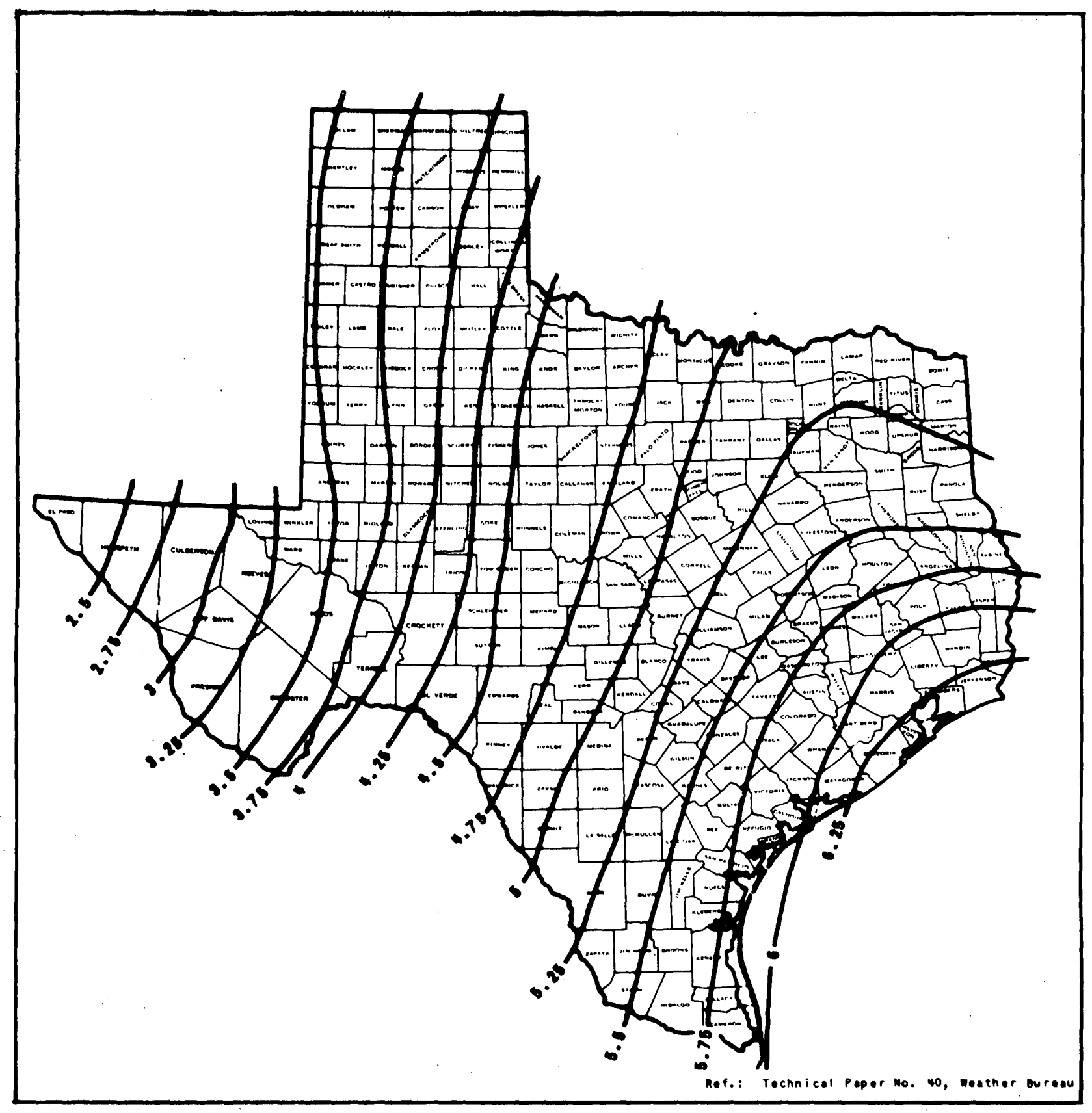

Figure 0.-Maximum three-hour rainfall, 50-year frequency in Texas 


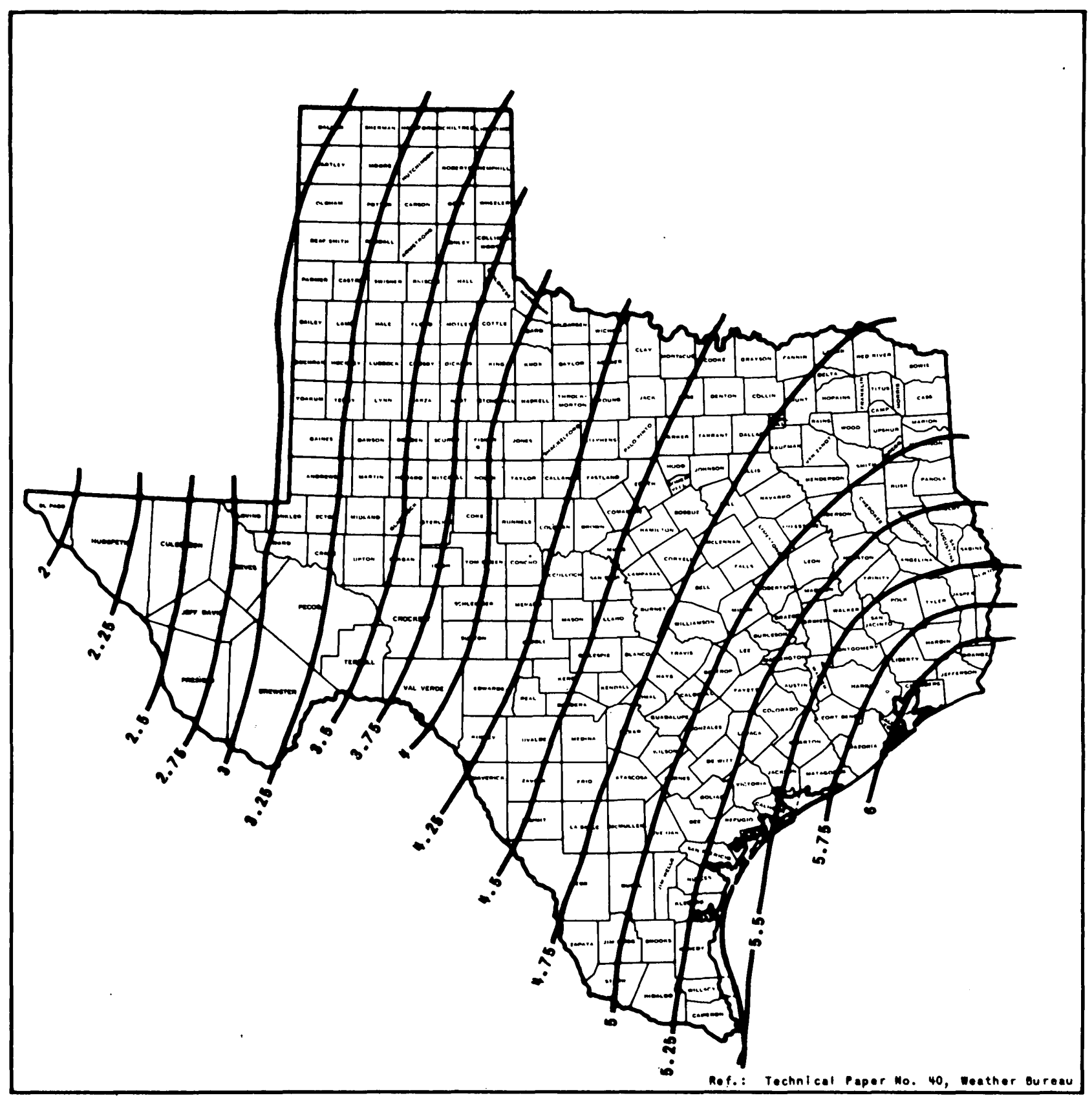

Figure 9.- Maximum six-hour rainfall, 10-year frequency in Texas 


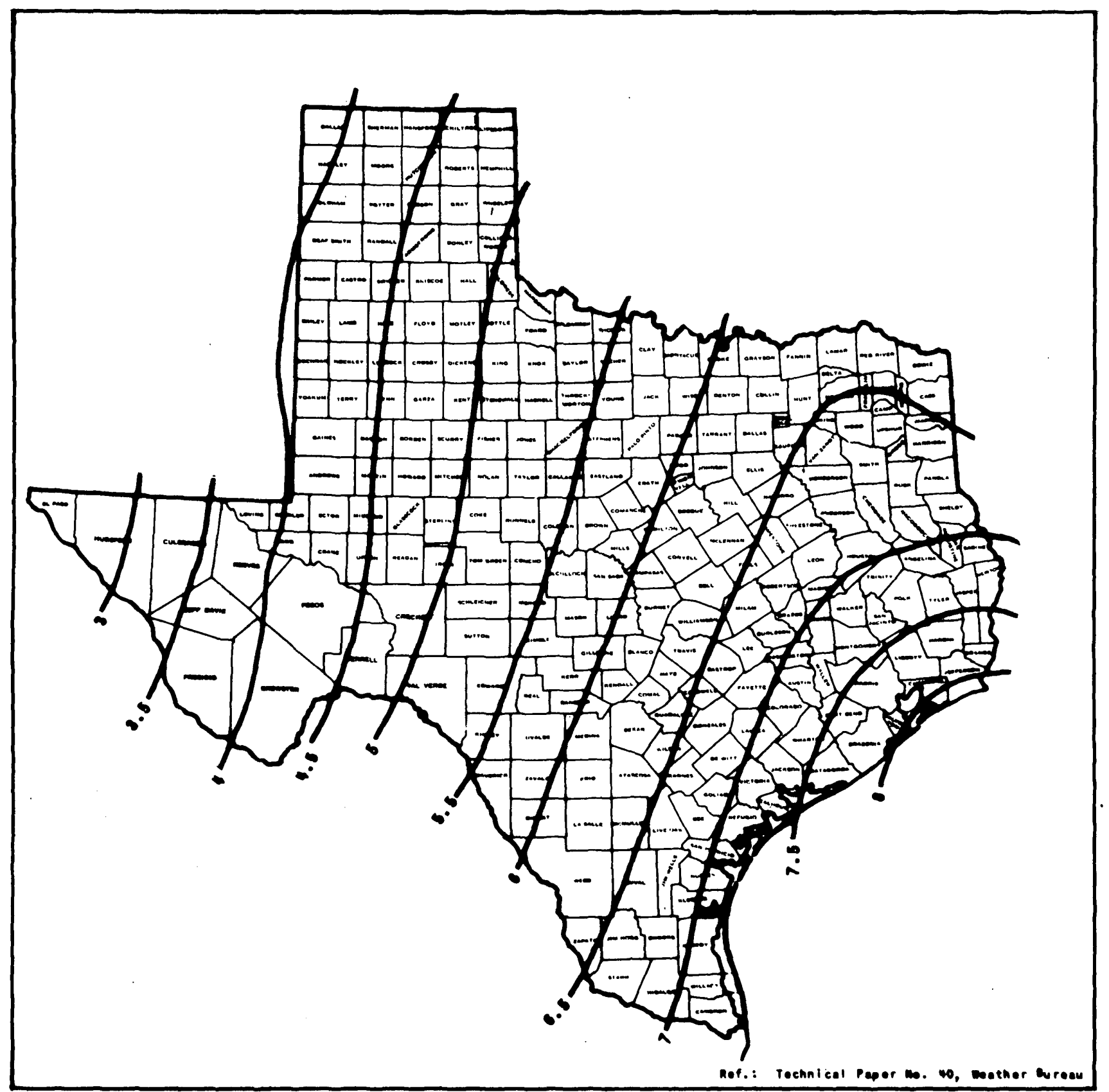

Flgure 10.-Maximum six-houp roinfall, 50-yeer frequency in Texes 


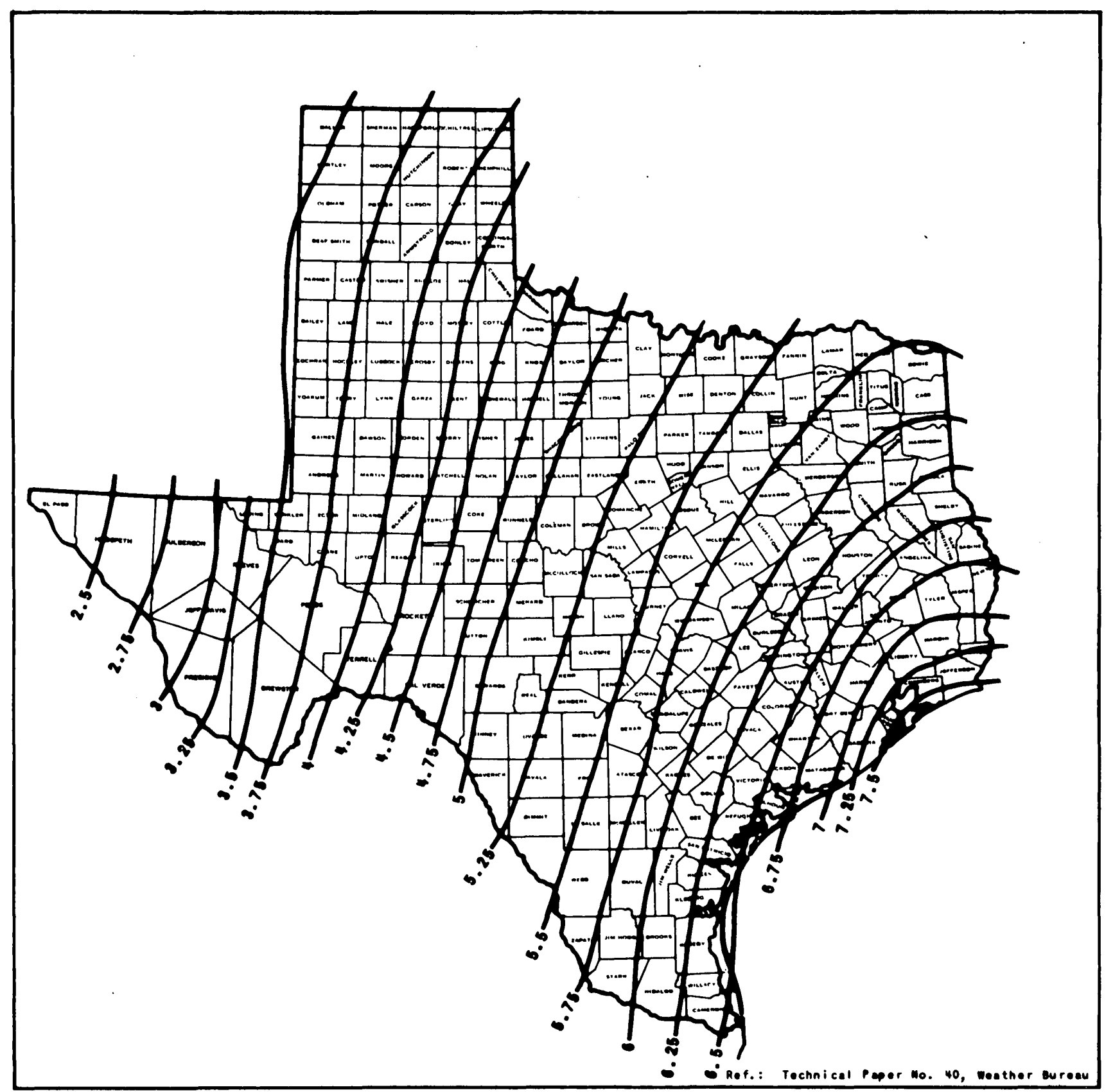

Flgure 11.-Moximum 12-hour rainfoll, 10-year frequency in Texos 


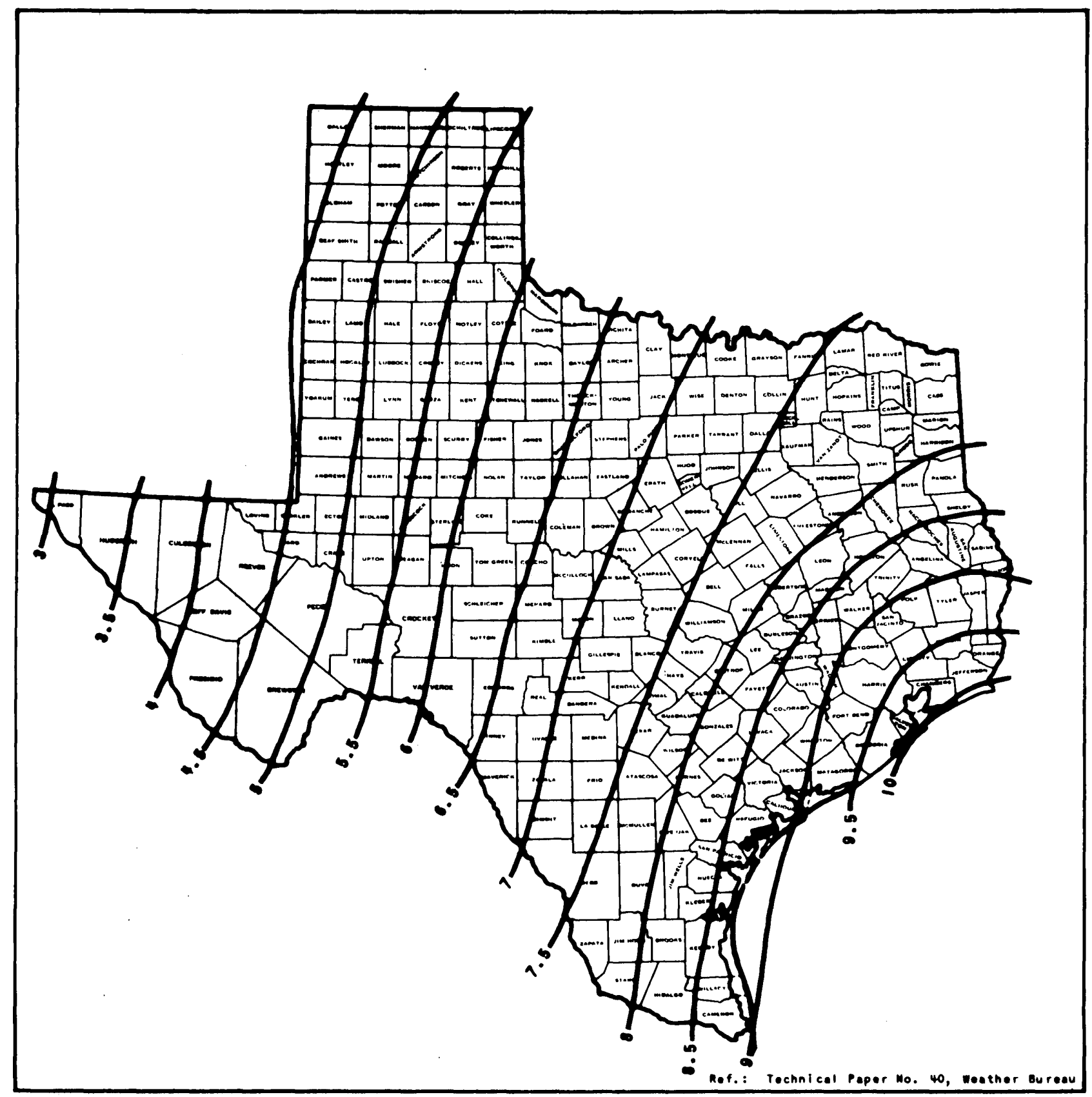

Flgure 12.-Moximum 12-hour rainfall, 50-yeor frequency in Texes 


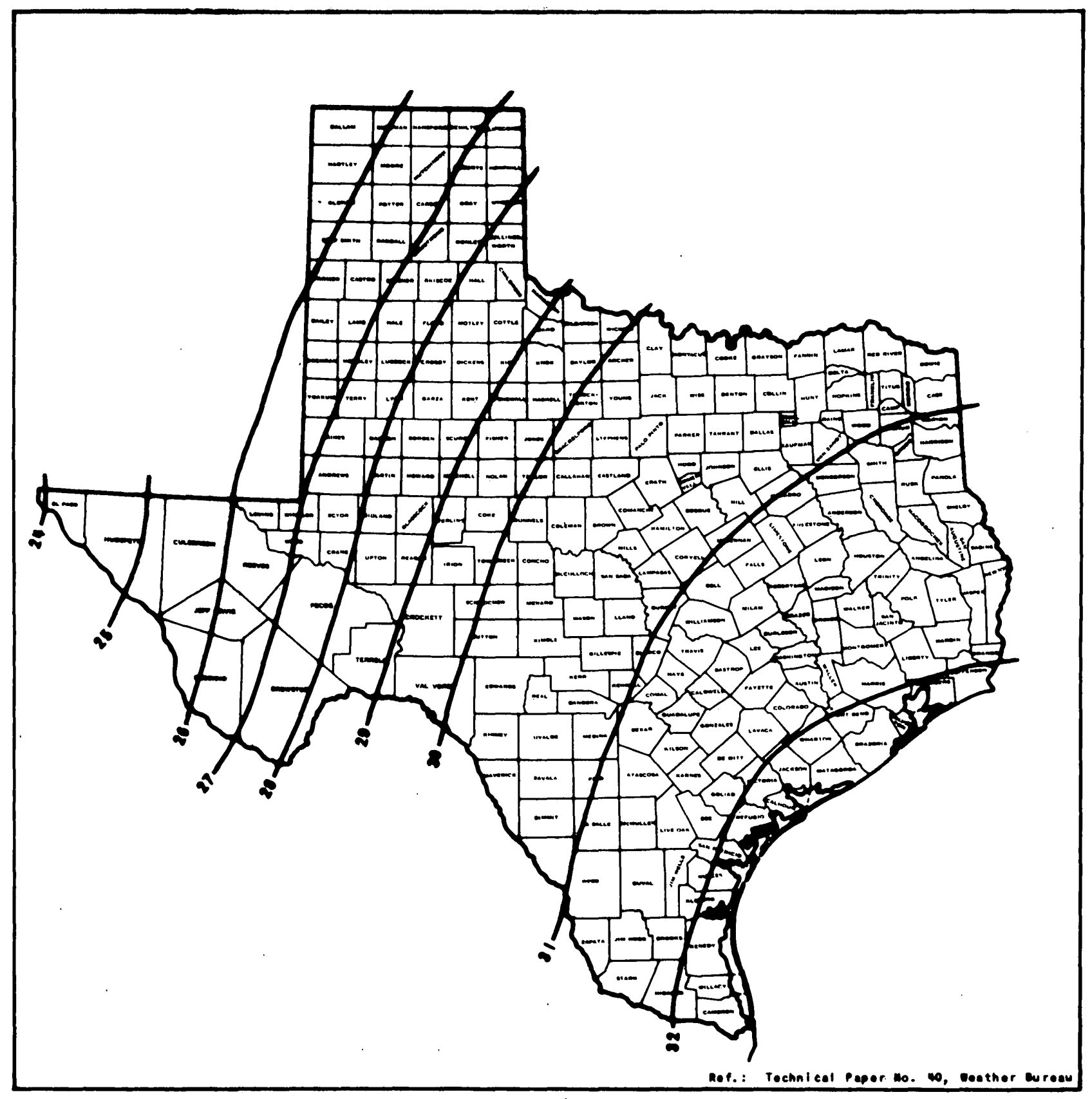

Flgure 13.-Proboble maximum 8-hour preciplifotion (inchẹ) for 10 squore miles in Texas 


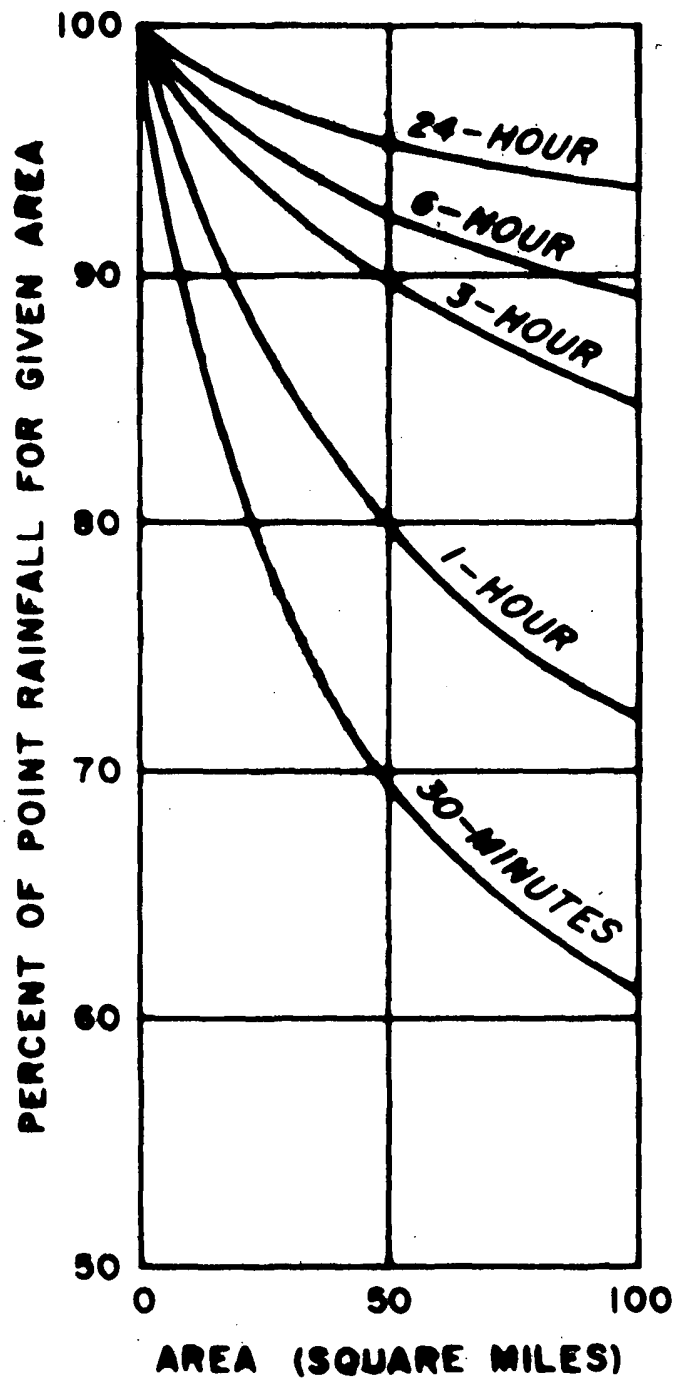

FIGUARE 14.-Aree-depin rainfall curmes 
Benson offers the most promising approach to evaluating magnitude and frequency of floods on small streams in Texas. He has shown that peak discharge for a selected frequency in Texas (excluding Red River drainage) can be described by certain meteorologic and basin characteristics. In general, these characteristics can be called hydrologic factors. Analyses of the data led to the conclusion that the following were the most important factors to be considered:

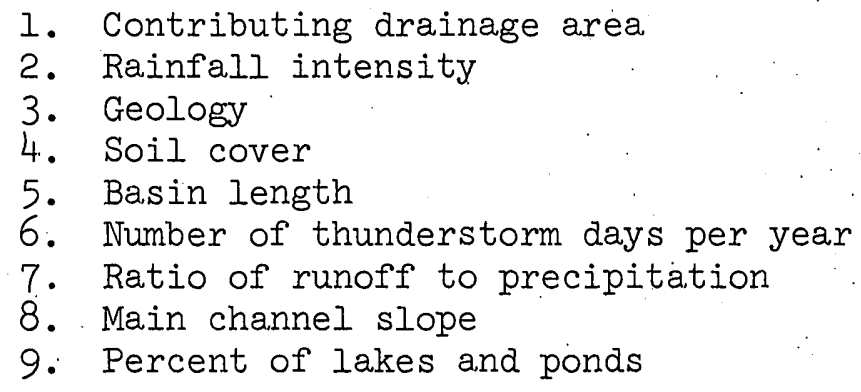

Chow (1962); Potter (1961), and others have endeavored to utilize hydrologic factors as a means of predicting peak rates of runoff on small streams. However, the lack of data necessary to verify the methods requires that caution be used when applying these methods to small streams in Texas. The cooperative program between the Geological Survey and the Texas Highway Department is designed to overcome this lack of data.

\section{SELECTED REFERENCES}

Benson, M. A., 1960, Evolution of methods for evaluating the occurrences of floods: U. S. Geol. Survey Water Supply Paper 1580-A, $30 \mathrm{p}$. , 1964, Factors affecting the occurrence of floods in the Southwest: U. S. Geol. Survey Water Supply Paper 1580-D, 72 p., I pl.

Breeding, S. D., 1948, Flood of September 1946 at San Antonio, Tex.: U. S. Geol. Survey Circular 32, 19 p.

Chow, V. T., 1962, Hydrologic determination of waterway areas for the design of drainage structures in small drainage basins: Univ. of Ill. Engr. Exp. Sta. Bull. 462, 104 p.

Dalrymple, Tate, 1960, Flood-frequency analyses: U. S. Geol. Survey Water Supply Paper 1543-A, 80 p.

Gilbert, C. R., and others, 1964, Hydrologic studies of small water- . sheds, Honey Creek basin, Collin and Grayson Counties, Texas, 1953-59: U. S. Geol. Survey Water Supply Paper 1779-F, 97 p., $3 \mathrm{pl}$.

Hershfield, D. M., 196l, Rainfall frequency atlas of the United States: U. S. Dept. of Commerce, Weather Bureau Tech. Paper 40, $115 \mathrm{p}$. Hudson, H. E., Stout, G. E., and Huff, F. A., 1954, Rainfall studies using rain gage networks and radar: Am. Soc. Civil Engineers Trans., v. 119, p. 248-273. 
Jennings, A. H., 1963; Maximum recorded United States point rainfall, for 5 minutes to 24 hours at 296 first-order stations: U. S. Dept. of Commerce, Weather Bureau Tech. Paper 2, 56 p.

Linsley, R. K., and Kohler, M. A., 1951, Variations in storm rainfall over small areas: Am. Geophys. Union Trans., v. 32, p. 245-270.

Patterson, J. L., 1963, Floods in Texas, magnitude and frequency of peak flows: Tex. Water Comm. Bull. 6311, $173 \mathrm{p}$. , 1964, Ma.gnitude and frequency of floods, U. S. Part 7: U.S. Geol. Survey Water Supply Paper 1681. , 1965, Magnitude and frequency of floods, U.S. Part 8: U.S. Geol. Survey Water Supply Paper 1682.

Potter, W. D., 1961, Peak rates of runoff from small watersheds:

U. S. Dept. of Commerce, Bureau of Pub. Roads Hydraulics Design Series No. 2, $35 \mathrm{p}$.

U. S. Geological Survey, 1960, Compilation of records of surface waters of the United States through September 1950, Part 8, Western Gulf of Mexico basins: U. S. Geol. Survey Water Supply Paper 1312, $633 \mathrm{p}$.

, 1964, Compilation of records of surface waters of the United States, October 1950 to September 1960, Part 8, Western Gulf of Mexico basins: U. S. Geol. Survey Water Supply Paper 1732, 574 p. , 1955, Compilation of records of surface waters of the United States through September 1950, Part 7, Lower Mississippi River basin: U. S. Geol. Survey Water Supply Paper 1311, 606.p. , 1964, Compilation of records of surface waters of the United States, October 1950 to September 1960, Part 7, Lower Mississippi River basin: U. S. Geol. Survey Water Supply Paper 1731, 552 p. , Destructive floods in the United States in 1904: U. S. Geol. Survey Water Supply Paper 147. , Destructive floods in the United States in 1905: U. S. Geol. Survey Water Supply Paper 162. , The flood in central Texas in September 1921: U. S. Geol. Survey Water Supply Paper 488. , Floods in the United States, frequency and magnitude: U. S. Geol. Survey Water Supply Paper 771. , Major Texa.s floods of 1935: U. S. Geol. Survey Wa.ter Supply Paper 796-G. , Major Texas floods of 1936: U. S. Geol. Survey Water Supply Paper 816. , Floods in Canadian and Pecos River basins of New Mexico, May and June 1937: U. S. Geol. Survey Wa.ter Supply Paper 842. , Maximum discharges at stream-measurement stations through September 1938: U. S. Geol. Survey Water Supply Paper 847. , Texas floods of 1938 and 1939: U. S. Geol. Survey Water Supply Paper 914. , Texas floods of 1940: U. S. Geol. Survey Water Supply Paper 1046.

, Summary of floods in the United States during 1950: U. S. Geol. Survey Water Supply Paper 1.137-I. 
U. S. Geological Survey, Floods of May 1951 in Western Oklahoma and northwestern Texas: U. S. Geol. Survey Water Supply Paper 1227-B. , Summary of floods in the United States during 1951: U. S. Geol. Survey Water Supply Paper 1227-D。 , Floods of September 1952 in Colorado and Guadalupe River basins, central Texas: U. S. Geol. Survey Water Supply Paper 1260-A. , Summary of floods in the United States during 1952: U. S. Geol. Survey Water Supply Paper 1260-F. , Floods of April-June 1953 in Louisiana and adjacent States:

U. S. Geol. Survey Water Supply Paper 1320-C. , Summary of floods in the United States during 1953: U. S. Geol. Survey Water Supply Paper 1320-E. , Summary of floods in the United States during 19.54: U. S. Geol. Survey Water Supply Paper 1370-C.

, 1949, Flood of May 17, 1949 at Fort Worth, Tex. Spec. Report 40. 
Table 1.--Peak discharge at miscellaneous sites

\begin{tabular}{|c|c|c|c|c|c|c|}
\hline \multirow[b]{2}{*}{$\begin{array}{l}\text { Map } \\
\text { No. }\end{array}$} & \multirow[b]{2}{*}{$\begin{array}{c}\text { Texas } \\
\text { Highway } \\
\text { District }\end{array}$} & \multirow[b]{2}{*}{ Stream and place of determination } & \multirow[b]{2}{*}{$\begin{array}{l}\text { Drainage } \\
\text { area } \\
(\mathrm{sq} \mathrm{mi})\end{array}$} & \multicolumn{3}{|c|}{ Peak discharge } \\
\hline & & & & Date & Cfs & $\begin{array}{l}\text { Cfs } \\
\text { per } \\
\text { sq mi }\end{array}$ \\
\hline \multicolumn{7}{|c|}{ Arkansas River basin } \\
\hline $\begin{array}{l}1 \\
2 \\
3\end{array}$ & $\begin{array}{l}4 \\
4 \\
4\end{array}$ & 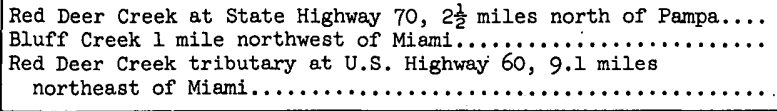 & $\begin{array}{r}3.4 \\
24.7 \\
1.0\end{array}$ & 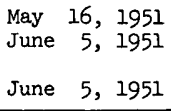 & $\begin{array}{r}3,430 \\
10,900 \\
1,610\end{array}$ & $\begin{array}{r}1,010 \\
441 \\
1,610\end{array}$ \\
\hline \multicolumn{7}{|c|}{ Red River basin } \\
\hline $\begin{array}{l}4 \\
5 \\
6\end{array}$ & $\begin{array}{r}25 \\
25 \\
4\end{array}$ & 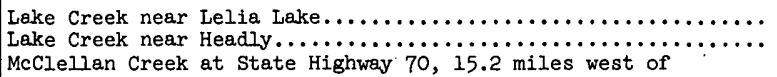 & $\begin{array}{l}48.6 \\
68.5\end{array}$ & $\begin{array}{l}\text { June } 15,1938 \\
\text { June } 15,1938\end{array}$ & $\begin{array}{r}40,800 \\
64,700\end{array}$ & $\begin{array}{l}840 \\
945\end{array}$ \\
\hline & & 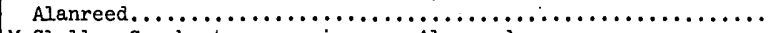 & 62 & 16,1951 & 8,720 & 140 \\
\hline 7 & 4 & McClellan Creek at reservoir near Alanreed............ & 86 & 16,1951 & 10,100 & 118 \\
\hline 8 & 4 & 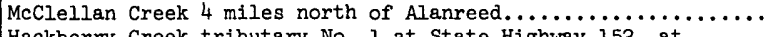 & 90 & June 8,1937 & 11,900 & 132 \\
\hline 9 & 25 & 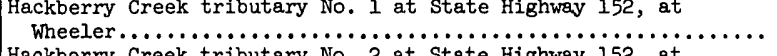 & 2.0 & June 5,1951 & 1,460 & 730 \\
\hline 10 & 25 & $\begin{array}{l}\text { Hackberry Creek tributary No. } 2 \text { at State Highway 152, at } \\
\text { Wheeler } . \ldots \ldots \ldots \ldots \ldots \ldots \ldots \ldots \ldots \ldots \ldots \ldots \ldots \ldots \ldots \ldots \ldots \ldots \ldots \ldots \ldots \ldots \ldots \ldots \ldots \ldots \ldots \ldots \ldots \ldots \ldots \ldots\end{array}$ & 1.2 & June 5,1951 & 2,340 & 1,950 \\
\hline 11 & 25 & Hackberry Creek tributary No. 3 half a mile upstream from. State & & & ? & \\
\hline 12 & 25 & 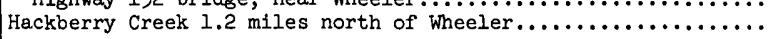 & 12.1 & June 5,1951 & 5,560 & 460 \\
\hline
\end{tabular}

\begin{tabular}{|c|c|c|c|c|c|c|}
\hline 13 & 11 & 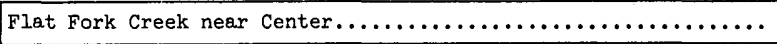 & 58 & July 24, 1933 & 42,200 & 728 \\
\hline \multicolumn{7}{|c|}{ Trinity River basin } \\
\hline $\begin{array}{l}14 \\
15 \\
16 \\
17 \\
18 \\
19\end{array}$ & $\begin{array}{r}2 \\
2 \\
18 \\
18 \\
18 \\
18\end{array}$ & 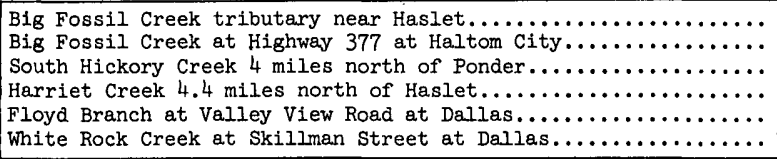 & $\begin{array}{l}0.92 \\
42.8 \\
23.0 \\
14.3 \\
3.18 \\
73.1\end{array}$ & $\begin{array}{l}\text { Sept. 7, } 1962 \\
\text { Sept. 7, } 1962 \\
\text { Sept. 7, } 1962 \\
\text { Sept. 7, } 1962 \\
\text { Oct. 8, } 1962 \\
\text { Sept.21, } 1964\end{array}$ & $\begin{array}{r}878 \\
31,800 \\
18,400 \\
14,100 \\
4,030 \\
42,300\end{array}$ & $\begin{array}{r}955 \\
742 \\
800 \\
987 \\
1,270 \\
578\end{array}$ \\
\hline \multicolumn{7}{|c|}{ Brazos River basin } \\
\hline \begin{tabular}{l|}
20 \\
21 \\
22 \\
23 \\
24 \\
25 \\
26 \\
27 \\
28 \\
29 \\
30 \\
31 \\
32
\end{tabular} & $\begin{array}{r}20 \\
8 \\
2 \\
2 \\
2 \\
9 \\
2 \\
2 \\
9 \\
9 \\
9 \\
23 \\
23\end{array}$ & 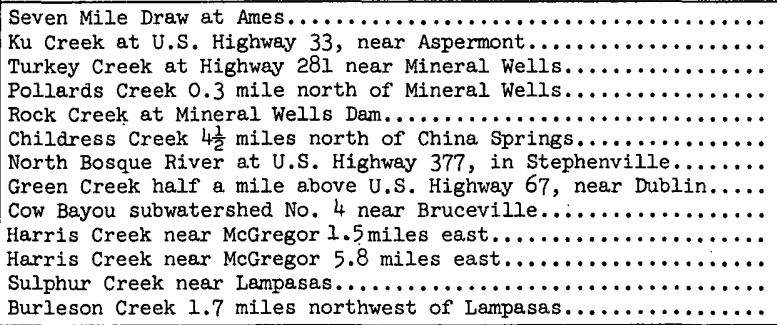 & $\begin{array}{c}2.4 \\
3.2 \\
9.66 \\
3.84 \\
74.4 \\
79 \\
93.3 \\
11.6 \\
5.25 \\
8.85 \\
20.9 \\
78.0 \\
7.4\end{array}$ & \begin{tabular}{|l} 
Sept.26, 1936 \\
Sept.25, 1955 \\
July 27, 1962 \\
July 27, 1962 \\
July 27, 1962 \\
Sept.26, 1936 \\
May 23, 1952 \\
May 23, 1952 \\
May 11, 1957 \\
June 16, 1964 \\
June 16, 1964 \\
May 12, 1957 \\
May 12, 1957
\end{tabular} & $\begin{array}{r}5,140 \\
3,000 \\
4,300 \\
12,100 \\
15,810 \\
47,000 \\
40,000 \\
18,900 \\
6,900 \\
10,800 \\
22,100 \\
65,300 \\
14,300\end{array}$ & $\begin{array}{r}2,140 \\
938 \\
445 \\
316 \\
213 \\
595 \\
429 \\
1,630 \\
1,310 \\
1,220 \\
1,060 \\
837 \\
1,930\end{array}$ \\
\hline
\end{tabular}

Colorado River basin

\begin{tabular}{|c|c|c|c|c|c|c|}
\hline & & & & & & \\
\hline $\begin{array}{l}33 \\
34 \\
35\end{array}$ & $\begin{array}{l}7 \\
7 \\
7\end{array}$ & $\begin{array}{l}\text { Mountain Creek at Mountain Creek Reservoir at Robert Lee....... } \\
\text { Cow Creek at briage on State Highway } 158 \text {, near Bronte } \ldots \ldots \cdots \cdots \\
\text { Pecan Creek } 2.3 \text { miles above mouth, } 10 \text { miles south of } \operatorname{San}\end{array}$ & $\begin{array}{r}25.5 \\
6.3\end{array}$ & $\begin{array}{l}\text { Aug. 19, } 1953 \\
\text { Aug. } 19,1953\end{array}$ & $\begin{array}{r}16,700 \\
5,200\end{array}$ & 825 \\
\hline 36 & 7 & 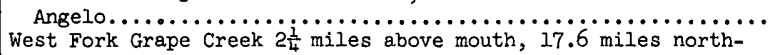 & 81 & Sept.15, 1936 & 30,500 & 377 \\
\hline 37 & 7 & 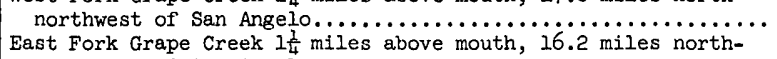 & 17 & Sept.17, 1936 & 14,200 & 835 \\
\hline 38 & 7 & $\begin{array}{l}\text { northwest of San Angelo } \ldots \ldots \ldots \ldots \ldots \ldots \ldots \ldots \ldots \ldots \ldots \ldots \ldots \ldots \\
\text { Grape Creek } I \text { mile below confluence of East and West Forks, }\end{array}$ & 32 & Sept.17, 1936 & 23,500 & 34 \\
\hline 39 & 7 & 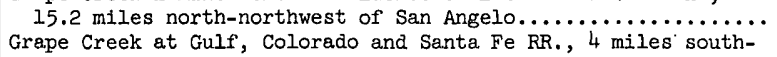 & 53 & Sept.17, 1936 & 31,800 & 0 \\
\hline 40 & 7 & 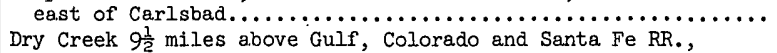 & 79 & Sept.17, 1936 & 45,600 & 577 \\
\hline 41 & 7 & 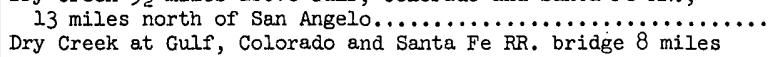 & 14 & Sept.17, 1936 & 24,600 & 1,760 \\
\hline & & northwest of San Angelo.......... & 48 & Sept.17, 1936 & 19,200 & 400 \\
\hline $\begin{array}{l}42 \\
43\end{array}$ & $\begin{array}{l}8 \\
8\end{array}$ & 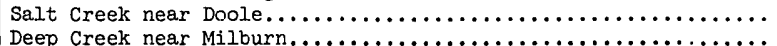 & 88.2 & July 23,1938 & 20,400 & 231 \\
\hline 正 & 7 & 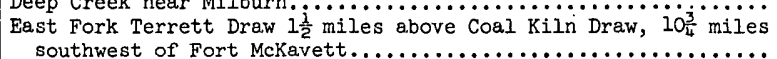 & 39.2 & JuLy 23,1938 & 0 & 8 \\
\hline & 7 & East Fork Terrett Draw a quarter of a mile below Coal Kiln & & (2) & 0 & \\
\hline 46 & 7 & $\begin{array}{l}\text { Draw, } 8 \frac{1}{4} \text { miles southwest of Fort McKavett } \ldots \ldots \ldots \ldots \ldots \ldots \ldots \\
\text { West Fork Terrett Draw } 1 \text { mile above mouth, } 6 \frac{1}{4} \text { miles southwest }\end{array}$ & 33 & Sept.16, 1936 & 18,700 & 567 \\
\hline 47 & 7 & 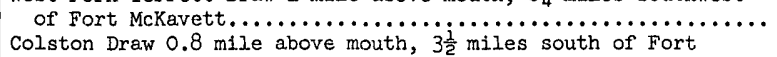 & 21 & Sept.16, 1936 & 5,880 & 280 \\
\hline & & 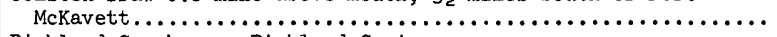 & 24 & Sept.16, 1936 & & 417 \\
\hline $\begin{array}{l}48 \\
49\end{array}$ & $\begin{array}{l}23 \\
23\end{array}$ & 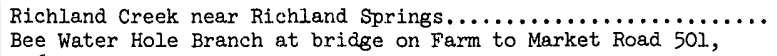 & 72. & July 23, 1938 & 61,000 & 843 \\
\hline 50 & 7 & 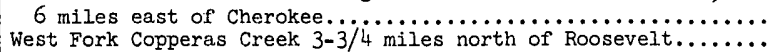 & $\begin{array}{r}4 . \\
81 .\end{array}$ & $\begin{array}{l}\text { Sept.10, } 1952 \\
\text { Sept.16, } 1936\end{array}$ & $\begin{array}{r}2,850 \\
50,400\end{array}$ & $\begin{array}{l}606 \\
622\end{array}$ \\
\hline 5. & 7 & 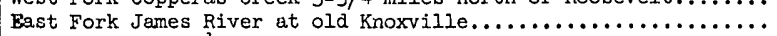 & 60.8 & July 1, 1932 & 105,000 & 1,730 \\
\hline 5 & 14 & Six Mile Creek $5 \frac{1}{2}$ miles west of Llano. & 24. & Sept.10, 1952 & 500 & 429 \\
\hline 53 & 14 & Johnson Creek at bridge on State Highway 29, near Llano. & 48. & Sept.11, 1952 & 200 & 252 \\
\hline 54 & 14 & Pecan Creek at Smathers Ranch, 6 miles northwest of Llano.. & 47.7 & Sept.11, 1952 & 11,900 & 249 \\
\hline & 14 & 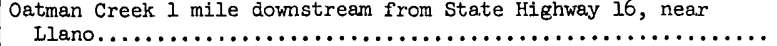 & 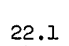 & 952 & 60 & 451 \\
\hline 56 & 14 & Wrights (or Mitcheil) Creek 3 & 14. & Sept.11, 1952 & & 460 \\
\hline 57 & 14 & 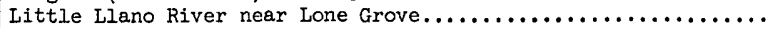 & 52.0 & Sept.10, 1952 & 21,800 & 419 \\
\hline
\end{tabular}


Table 1.--Peak discharge at miscellaneous sites--Cont1nued

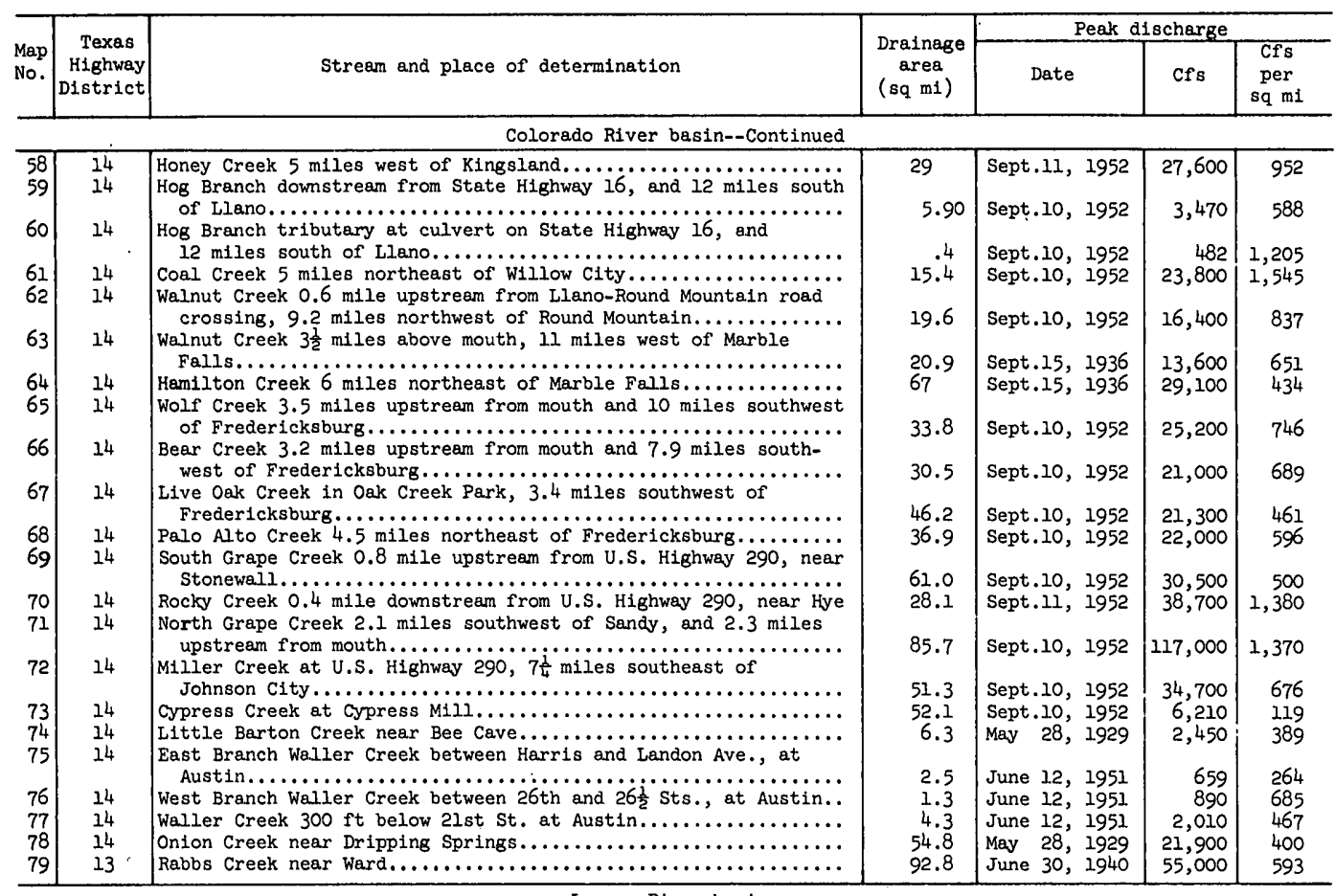

\begin{tabular}{|c|c|c|c|c|c|c|}
\hline 80 & 13 & Youngs Branch 2 miles east of Moulton... & 6.8 & June 30,1940 & 8,900 & 1,310 \\
\hline $\begin{array}{l}81 \\
82 \\
83 \\
84 \\
85\end{array}$ & $\begin{array}{l}15 \\
15 \\
15 \\
15 \\
15\end{array}$ & 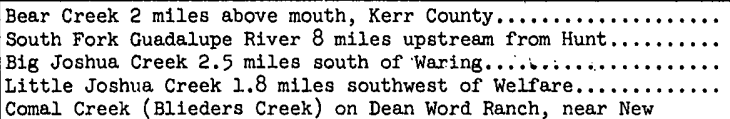 & $\begin{array}{r}29.1 \\
60.3 \\
17.8 \\
8.94\end{array}$ & $\begin{array}{l}\text { July } 1,1932 \\
\text { July } 1,1932 \\
\text { Sept.10, } 1952 \\
\text { Sept.10, } 1952\end{array}$ & $\begin{array}{l}17,200 \\
84,300 \\
30,900 \\
12,800\end{array}$ & $\begin{array}{l}590 \\
1,400 \\
1,740 \\
1,430\end{array}$ \\
\hline $\begin{array}{l}86 \\
87 \\
88\end{array}$ & $\begin{array}{l}15 \\
14 \\
14\end{array}$ & 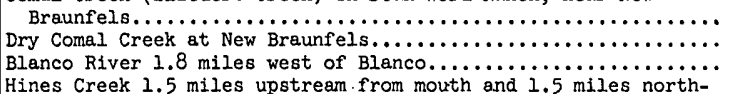 & $\begin{array}{l}17.9 \\
94 \\
93.5\end{array}$ & $\begin{array}{l}\text { Sept.11, } 1952 \\
\text { Sept.11, } 1952 \\
\text { Sept.11, } 1952\end{array}$ & $\begin{array}{r}8,480 \\
35,000 \\
61,900\end{array}$ & $\begin{array}{l}473 \\
373 \\
663\end{array}$ \\
\hline 89 & 14 & 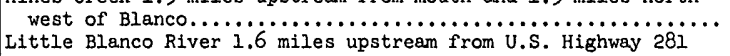 & 2.92 & Sept.10, 1952 & 5,430 & 1,860 \\
\hline 90 & 14 & 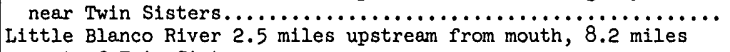 & 21.9 & Sept.10, 1952 & 19,900 & 910 \\
\hline $\begin{array}{l}91 \\
92 \\
93\end{array}$ & $\begin{array}{l}14 \\
13 \\
13\end{array}$ & 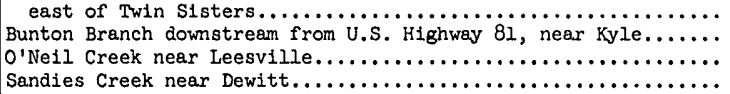 & $\begin{array}{l}60.3 \\
4.12 \\
30 \\
95\end{array}$ & $\begin{array}{l}\text { Sept.10, } 1952 \\
\text { June } 30,1936 \\
\text { July } 1,1936 \\
\text { July } 1,1936\end{array}$ & $\begin{array}{l}41,000 \\
1.3,800 \\
30,000 \\
54,300\end{array}$ & $\begin{array}{r}680 \\
3,350 \\
1,000 \\
572\end{array}$ \\
\hline
\end{tabular}

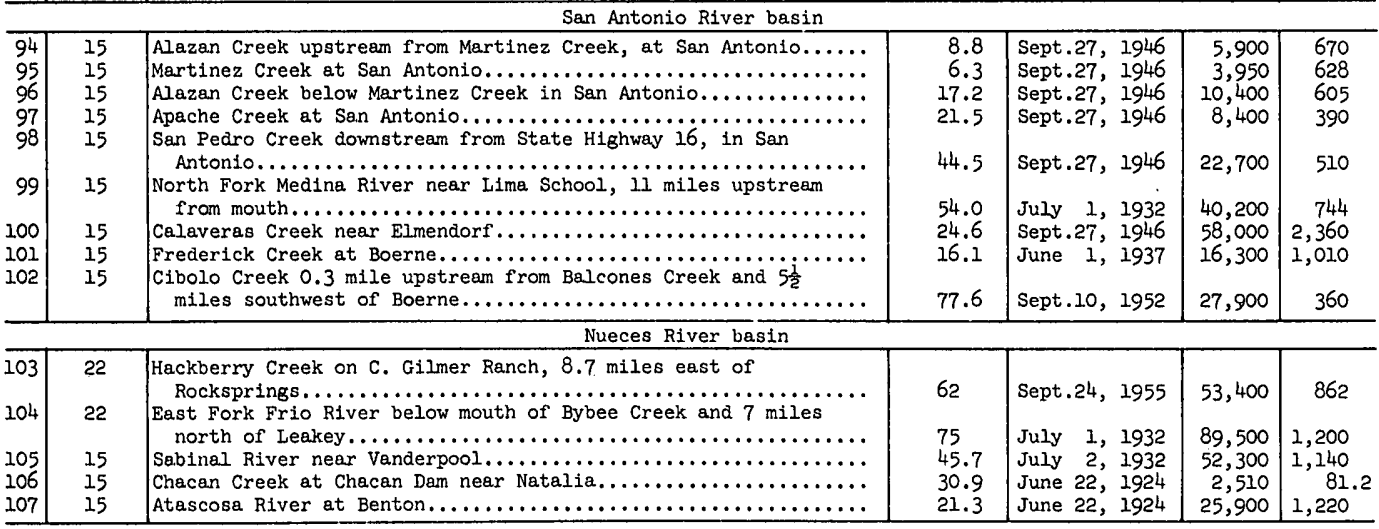

Minor Coastal basins

\begin{tabular}{|c|c|c|c|c|c|c|}
\hline $\begin{array}{l}108 \\
109\end{array}$ & $\begin{array}{l}16 \\
21\end{array}$ & 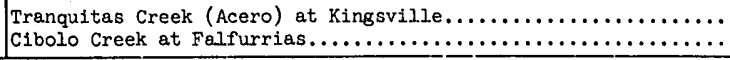 & $\begin{array}{l}54.3 \\
95\end{array}$ & $\begin{array}{l}\text { Sept.15, } 1951 \\
\text { Sept.15, } 1951\end{array}$ & $\begin{array}{l}4,790 \\
3,460 \\
\end{array}$ & $\begin{array}{l}88.2 \\
36.4\end{array}$ \\
\hline \multicolumn{7}{|c|}{ Rio Grande basin } \\
\hline $\begin{array}{l}110 \\
111 \\
112\end{array}$ & $\begin{array}{r}6 \\
22 \\
22\end{array}$ & 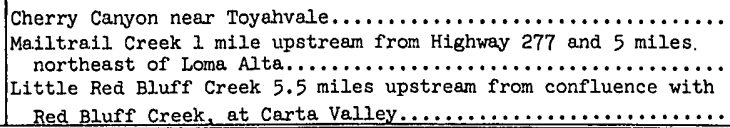 & $\begin{array}{l}70.9 \\
75.3 \\
10.3\end{array}$ & $\begin{array}{l}\text { Sept.29, } 1932 \\
\text { June 24, } 1948 \\
\text { June 24, } 1948\end{array}$ & $\begin{array}{r}5,320 \\
170,000 \\
30,000\end{array}$ & $\begin{array}{r}75.0 \\
2,260 \\
2,910\end{array}$ \\
\hline
\end{tabular}


STATION DATA

PEAK DISCHARGES AT GAGING STATIONS AND PARTIAL-RECORD STATIONS LISTED BY BASIN AND IN DOWNSTREAM ORDER 


\section{STATION DATA}

ARKANSAS RIVER BASIN

7-2274.50. Unnamed tributary (watershed W-1) of Middle Alamosa Creek near Vega, Tex. (4)

Location.--Lat $35^{\circ} 18^{\prime}$, long $102^{\circ} 25^{\prime}, 5$ miles north of Vega, Oldham County .

Drainage area.--0.202 sq mi.

Gage.--Recording.

Remarks.--Records furnished by U. S. Department of Agriculture, Agricultural Research Service. Only annual (calendar year) peaks are shown.

\begin{tabular}{l|c|c|c||c|c|c|c}
\hline $\begin{array}{l}\text { Calen- } \\
\text { dar } \\
\text { year }\end{array}$ & Date & $\begin{array}{c}\text { Gage } \\
\text { height } \\
\text { (feet) }\end{array}$ & $\begin{array}{c}\text { Discharge } \\
\text { (cfs) }\end{array}$ & $\begin{array}{c}\text { Calen- } \\
\text { dar } \\
\text { year }\end{array}$ & Date & $\begin{array}{c}\text { Gage } \\
\text { height } \\
\text { (feet) }\end{array}$ & $\begin{array}{c}\text { Discharge } \\
\text { (cfs) }\end{array}$ \\
\hline 1938 & May 30, 1938 & - & 107 & 1941 & May 20, 1941 & - & 29 \\
1939 & Apr. 5, 1939 & - & 258 & 1942 & July 8, 1943 & - & $($ a) \\
1940 & May 27, 1940 & - & 1.3 & 1943 & - & 139 \\
\hline
\end{tabular}

a Less than $0.1 \mathrm{cfs}$.

$$
\begin{gathered}
\text { 7-2274.55. Unnamed tributary (watershed W-2) of Middle Alamosa Creek } \\
\text { near Vega, Tex. (4) }
\end{gathered}
$$

Location.--Lat $35^{\circ} 20^{\prime}$, long $102^{\circ} 25^{\prime}$, 6 miles north of Vega, Oldham County.

Drainage area.--0.150 sq mi.

Gage.--Recording.

Remarks.--Records furnished by U. S. Department of Agriculture, Agricultural Research Service. Only annual (calendar year) peaks are shown.

\begin{tabular}{l|c|c|c||c|c|c|c}
\hline $\begin{array}{c}\text { Calen- } \\
\text { dar } \\
\text { year }\end{array}$ & Date & $\begin{array}{c}\text { Gage } \\
\text { height } \\
\text { (feet) }\end{array}$ & $\begin{array}{c}\text { Discharge } \\
\text { (cfs) }\end{array}$ & $\begin{array}{c}\text { Calen- } \\
\text { dar } \\
\text { year }\end{array}$ & Date & $\begin{array}{c}\text { Gage } \\
\text { height } \\
\text { (feet) }\end{array}$ & $\begin{array}{c}\text { Discharge } \\
\text { (cfs) }\end{array}$ \\
\hline 1938 & May 30, 1938 & - & 141 & 1941 & Aug. 23, 1941 & - & \\
1939 & July 26, 1939 & - & 64 & 1942 & Apr. 19, 1942 & - & 6 \\
1940 & May 27, 1940 & - & 15 & 1943 & May 26, 1943 & - & 6.8 \\
\hline
\end{tabular}


7-2980. North Tule Draw at reservolr, near Tul1a, Tex. (7)

Location.--Lat $34^{\circ} 33^{\prime}$, long $101^{\circ} 42^{\prime}$, at walkway to condu1t Intake valve, $250 \mathrm{ft}$ to left of concrete spillway, I mile upstream from mouth, and 3.2 miles northeast of Tulia, Swisher County. $\frac{\text { Drainage area }}{\text { to surface }}$--About $189 \mathrm{sq} \mathrm{ml}$, of which about $65 \mathrm{sq} \mathrm{ml}$ contributes directly

Gage.--Nonrecording prior to Nov, 26, 1940; recording thereafter. Prior to St (by barometer).
$\mathrm{ft}$, 1939 at $70.5 \mathrm{ft}$ higher. Altitude of present gage is 3,310

Stage-discharge relation.--Peak inflow is based on change in reservolr contents, How over splilway (computed from splilway rating curve), and computed flow through conduit.

Remarks. --Dam completed Jan. 15, 1939. Reservolr capac1ty, 654 acre-ft. No regulation upstream from reservolr. Only annual peaks are shown.

Peak stages and discharges

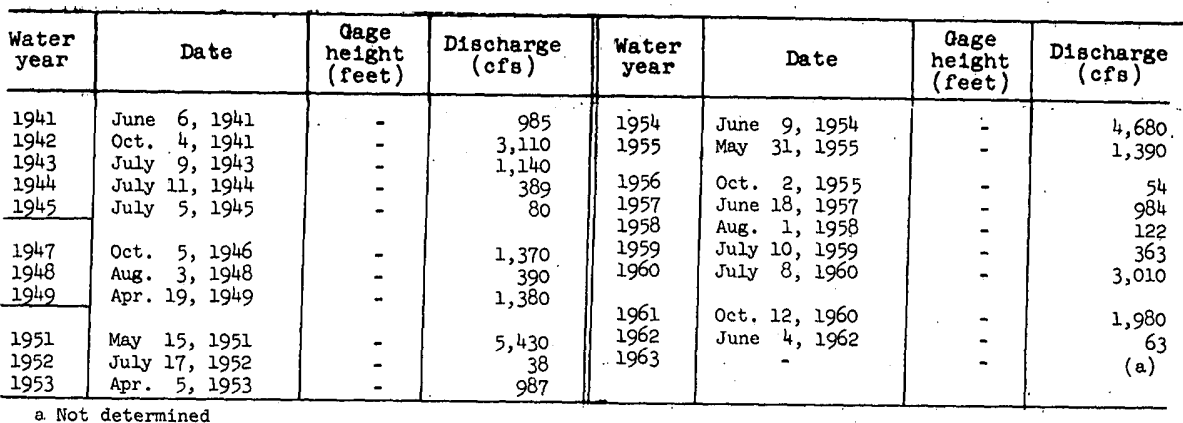

a. Not determined

7-3075. Quitaque Creek near Quitaque, Tex.(25)

Location.--Lat $34^{\circ} 14^{\prime}$, long $101^{\circ} 07^{\prime}$, on right bank about three-quarters of a mIle upstream from W. F. Saul's ranchhouse, I mile downstream from Wilson Creek, I $1 \frac{1}{2}$ miles upstream from Turkey Creek, 10 miles southwest of Quitaque, Briscoe county, and at mile 22.3 .

Dralnage area. --293 $\mathrm{sq} \mathrm{ml}$, of which about $35 \mathrm{sq} \mathrm{ml}$ contributes directly to surface runoff

Gage.--Recording gage and concrete control. Datum of gage 1s $2,633.91 \mathrm{ft}$ above mean sea level, datum of 1929.

Stage-discharge relation.--Defined by current-meter measurements below $70 \mathrm{cfs}$ and extended on basis of slope-area measurements at gage helghts $2.70,3.00$, 5.59 , and $8.62 \mathrm{ft}$.

Bankfull stage. $--9 \mathrm{ft}$.

Remarks.--Base for partial-duration series, $500 \mathrm{cfs}$.

\begin{tabular}{|c|c|c|c|c|c|c|c|}
\hline $\begin{array}{l}\text { Water } \\
\text { year }\end{array}$ & Date & $\begin{array}{c}\text { Gage } \\
\text { he1ght } \\
\text { (feet) }\end{array}$ & $\begin{array}{l}\text { D1scharge } \\
\text { (cfs) }\end{array}$ & $\begin{array}{l}\text { Water } \\
\text { year }\end{array}$ & Date & $\begin{array}{c}\text { Gage } \\
\text { he1ght } \\
\text { (feet) }\end{array}$ & $\begin{array}{c}\text { D1gcharge } \\
(\text { cfs })\end{array}$ \\
\hline 1946 & Sept.19, 1946 & 2.77 & 423 & 1954 & May 10,1954 & 4.11 & 970 \\
\hline 2947 & $\begin{array}{lr}\text { May } & 8,1947 \\
\text { May } & 10,1947\end{array}$ & $\begin{array}{l}3.57 \\
5.59\end{array}$ & $\begin{array}{r}720 \\
1,720\end{array}$ & & AUB. 23,1954 & 3.37 & 680 \\
\hline & May 16,1947 & 3.40 & 660 & 1955 & 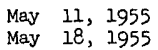 & $\begin{array}{l}3.75 \\
6.15\end{array}$ & $\begin{array}{l}1,040 \\
2,000\end{array}$ \\
\hline 1948 & Sept. 8, 1948 & 3.00 & 520 & & June 1,1955 & 6.47 & 2,290 \\
\hline 1949 & $\begin{array}{l}\text { May } 28,1949 \\
\text { June } 7,1949\end{array}$ & $\begin{array}{l}3.66 \\
3.35\end{array}$ & $\begin{array}{l}785 \\
640\end{array}$ & & June 28, 1955 & 8.62 & 4,470 \\
\hline 1950 & $\begin{array}{l}\text { June } 11,1950 \\
\text { July } 23,1950\end{array}$ & $\begin{array}{l}3.03 \\
3.20\end{array}$ & $\begin{array}{l}536 \\
600\end{array}$ & 1956 & $\begin{array}{l}\text { May } 27,1956 \\
\text { June } 17,1956\end{array}$ & $\begin{array}{l}3.28 \\
3.01\end{array}$ & $\begin{array}{l}700 \\
536\end{array}$ \\
\hline & Sept. 4, 1950 & 5.57 & 1,700 & 1957 & $\begin{array}{ll}\text { May } 11, & 1957 \\
\text { May } & 31,1957\end{array}$ & $\begin{array}{l}3.70 \\
7.50\end{array}$ & $\begin{array}{r}900 \\
2,900\end{array}$ \\
\hline 1951 & $\begin{array}{l}\text { May } 17,1951 \\
\text { Sept. 9, } 1951\end{array}$ & $\begin{array}{l}3.08 \\
3.69\end{array}$ & $\begin{array}{l}556 \\
780\end{array}$ & & Aug. 4, 1957 & 6.33 & 6,060 \\
\hline 1952 & July 14, 1952 & 2.07 & 152 & 1930 & June 23, 1950 & 2.32 & 812 \\
\hline 1953 & Aug. 15, 1953 & 4.67 & 1,240 & 1959 & 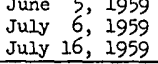 & $\begin{array}{l}2.09 \\
2.82 \\
3.21 \\
\end{array}$ & $\begin{array}{r}613 \\
1,220 \\
1,120 \\
\end{array}$ \\
\hline
\end{tabular}


RED RIVER BASIN

7-3326. Bois d'Arc Creek near Randolph, Tex. (1)

Location.--Lat $33^{\circ} 28^{\prime} 30^{\prime \prime}$, long $96^{\circ} 12^{\prime} 55^{\prime \prime}$, on right bank at downstream side of bridge on Farm Road 1281, 2.3 miles upstream from Henson Creek, and 2.4 miles east of Randolph, Fannin County.

Drainage area. --72 sq $\mathrm{mi}$.

Gage.--Recording. Datum of gage is $564.38 \mathrm{ft}$ above mean sea level, datum of 1929.

Stage-discharge relation.--Defined by current-meter measurements below 1,100 cfs and above by slopearea measurement of $7,700 \mathrm{cfs}$.

Bankfull stage.--2l ft.

Historical data.--Maximum stage about $24.6 \mathrm{ft}$ occurred about 1935, from information by State Highway Department.

Remarks.--Base for partial-duration series, 1,500 cfs. Rain gage at site.

Peak stages and discharges

\begin{tabular}{c|c|c|c||c|c|c|c}
\hline $\begin{array}{c}\text { Water } \\
\text { year }\end{array}$ & Date & $\begin{array}{c}\text { Gage } \\
\text { height } \\
\text { (feet) }\end{array}$ & $\begin{array}{c}\text { Discharge } \\
\text { (cfs) }\end{array}$ & $\begin{array}{c}\text { Water } \\
\text { vear }\end{array}$ & Date & $\begin{array}{c}\text { Gage } \\
\text { height } \\
\text { (feet) }\end{array}$ & $\begin{array}{c}\text { Discharge } \\
\text { (cfs) }\end{array}$ \\
\hline 1963 & Apr. 28, 1963 & 9.85 & 3,600 & & & & \\
\hline
\end{tabular}

\section{7-3368. Pecan Bayou near Clarksville, Tex. (1)}

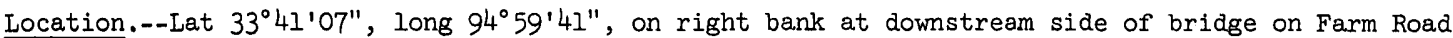
II59, 0.2 mile downstream from Tanyard Bayou, 4.3 miles upstream from Little White Oak Creek, and 6.0 miles northeast of Clarksville, Red River County.

Drainage area.--100 sq mi.

Gage.--Recording. Datum of gage is $365.00 \mathrm{ft}$ above mean sea level, datum of 1929.

Stage-discharge relation.--Defined by current-meter measurements.

Bankfull stage.--10 ft.

Historical data.--Maximum stage since at least 1910, about $12 \mathrm{ft}$ in 1957, from information by local residents.

Remarks.--Base for partial-duration series, $800 \mathrm{cfs}$.

Peak stages and discharges

\begin{tabular}{|c|c|c|c|c|c|c|c|}
\hline $\begin{array}{r}\text { Water } \\
\text { year }\end{array}$ & Date & $\begin{array}{c}\text { Gage } \\
\text { height } \\
\text { (feet) }\end{array}$ & $\begin{array}{c}\text { Discharge } \\
\text { (cfs) }\end{array}$ & $\begin{array}{r}\text { Water } \\
\text { year }\end{array}$ & Date & $\begin{array}{c}\text { Gage } \\
\text { height } \\
\text { (feet) }\end{array}$ & $\begin{array}{l}\text { Discharge } \\
\text { (cfs) }\end{array}$ \\
\hline 1962 & $\begin{array}{ll}\text { Jan. } 27, & 1962 \\
\text { Apr. 1, } 1962 \\
\text { Apr. 29, } 1962\end{array}$ & $\begin{array}{l}7.00 \\
6.03 \\
6.20\end{array}$ & $\begin{array}{r}1,980 \\
851 \\
980\end{array}$ & $\begin{array}{l}1962 \\
1963\end{array}$ & $\begin{array}{l}\text { May 1, } 1962 \\
\text { Nov. 27, } 1962\end{array}$ & $\begin{array}{l}6.15 \\
5.81\end{array}$ & $\begin{array}{l}940 \\
706\end{array}$ \\
\hline
\end{tabular}


7-3450. Boggy Creek near Dalngerfield, Tex.(19)

Location.--Lat 33.02105", long 94.47110", on right bank at downstream side of bridge on State Highway 11, a quarter of a mile upstream from Louisiana \& Arkansas Rallway Co. bridge, 3.8 miles west of Dalngerfield, Morris County, 9 miles upstream from mouth, and at mile 11.5.

Drainage area. $--72 \mathrm{sq} \mathrm{ml}$.

Gage.--Recording. Prior to 0ct. 1, 1954, at site 1,700 ft downstream at present datum. Datum of gage is $258.41 \mathrm{ft}$ above mean sea level, datum of 1929 .

Stage-discharge relation. --Defined by current-meter measurements.

Bankfull stage. $--10 \mathrm{ft}$.

H1storical data. - The flood in January 1938 is the second highest since at least 1900 , from Information by local residents.

Remarks. --Base for partial-duration series, 1,000 cfs.

Peak stages and discharges

\begin{tabular}{|c|c|c|c|c|c|c|c|}
\hline $\begin{array}{l}\text { Water } \\
\text { year }\end{array}$ & Date & $\begin{array}{c}\text { Gage } \\
\text { he1ght } \\
\text { (feet) }\end{array}$ & $\begin{array}{c}\text { Discharge } \\
(\mathrm{cfs})\end{array}$ & $\begin{array}{l}\text { Water } \\
\text { year }\end{array}$ & Date & $\begin{array}{c}\text { Gage } \\
\text { helght } \\
\text { (feet) }\end{array}$ & $\begin{array}{c}\text { D1scharge } \\
\text { (cfs) }\end{array}$ \\
\hline 1938 & January 1938 & a.16 & - & 1950 & Mar. 13, 1950 & 9.36 & 1,260 \\
\hline 1944 & $\begin{array}{l}\text { Feb. } 29,1944 \\
\text { Mar. 19, } 1944 \\
\text { Apr. } 9,1944 \\
\text { May } 2,1944 \\
\text { May } 27,1944\end{array}$ & $\begin{array}{r}9.33 \\
9.82 \\
9.66 \\
12.40 \\
9.93\end{array}$ & $\begin{array}{l}1,240 \\
1,880 \\
1,620 \\
9,650 \\
2,080\end{array}$ & & $\begin{array}{lll}\text { May } 7, & 1940 \\
\text { May } 14, & 1950 \\
\text { May } 31,1950 \\
\text { Sept.17, } 1950\end{array}$ & $\begin{array}{r}11.83 \\
11.23 \\
9.57 \\
12.97\end{array}$ & $\begin{array}{r}0,240 \\
7,250 \\
1,040 \\
1,510 \\
10,100\end{array}$ \\
\hline \multirow[t]{4}{*}{1945} & $\begin{array}{l}\text { Dec. 29, } 1944 \\
\text { Feb: 22, } 1945\end{array}$ & $\begin{array}{r}9.91 \\
10.68\end{array}$ & $\begin{array}{l}2,040 \\
4,080\end{array}$ & 1951 & $\begin{array}{l}\text { Feb. } 16,1951 \\
\text { Feb. 19, } 1951\end{array}$ & $\begin{array}{l}9.15 \\
9.20\end{array}$ & $\begin{array}{l}1,160 \\
1,210\end{array}$ \\
\hline & $\begin{array}{l}\text { Feb. 28, } 1945 \\
\text { Mar. } 4,1945 \\
\text { Mar. 20, } 1945 \\
\text { Mar. 30, } 1945\end{array}$ & $\begin{array}{r}10.25 \\
9.85 \\
9.60 \\
14.10\end{array}$ & $\begin{array}{r}1,820 \\
1,930 \\
1,540 \\
15,900\end{array}$ & $\begin{array}{l}1952 \\
\therefore\end{array}$ & $\begin{array}{l}\text { Apr. } 13,1952 \\
\text { Apr. 23, } 1952 \\
\text { May } 30,1952\end{array}$ & $\begin{array}{r}10.93 \\
10.68 \\
9.00\end{array}$ & $\begin{array}{l}4,610 \\
3,700 \\
1,070\end{array}$ \\
\hline & $\begin{array}{l}\text { Apr. } 2,1945 \\
\text { May 16, } 1945 \\
\text { June } 12,1945\end{array}$ & $\begin{array}{r}11.10 \\
9.18 \\
10.82\end{array}$ & $\begin{array}{l}5,070 \\
1,240 \\
5,010\end{array}$ & 1953 & $\begin{array}{l}\text { Apr. } 30,1953 \\
\text { May } 16,1953\end{array}$ & $\begin{array}{r}9.49 \\
11.05\end{array}$ & $\begin{array}{l}1,290 \\
4,290\end{array}$ \\
\hline & June 23,1945 & 10.15 & 2,960 & 1954 & May 30,1954 & 10.50 & 3,100 \\
\hline \multirow[t]{3}{*}{1946} & $\begin{array}{l}\text { Jan. } 10,1946 \\
\text { May } 1,1946\end{array}$ & $\begin{array}{l}9.28 \\
9.55\end{array}$ & $\begin{array}{l}1,000 \\
1,250\end{array}$ & 1955 & Mar. 22, 1955 & 10.80 & 2,540 \\
\hline & $\begin{array}{l}\text { May } 14,1946 \\
\text { May } 19,1946\end{array}$ & $\begin{array}{l}10.50 \\
10.20\end{array}$ & $\begin{array}{l}1,860 \\
2,120\end{array}$ & 1956 & Feb. 17, 1956 & 9.13 & 350 \\
\hline & June 1,1946 & 10.22 & 2,160 & 1957 & $\begin{array}{ll}\text { Apr. 24, } 1957 \\
\text { Apr. 27, } 1957\end{array}$ & $\begin{array}{l}11.34 \\
11.59\end{array}$ & $\begin{array}{l}2,160 \\
2,600\end{array}$ \\
\hline \multirow[t]{2}{*}{1947} & $\begin{array}{l}\text { Nov. } 7,1946 \\
\text { Nov. } 11,1946\end{array}$ & $\begin{array}{l}9.80 \\
9.65\end{array}$ & $\begin{array}{l}1,540 \\
1,360\end{array}$ & & June 23, 1957 & 10.53 & 1,260 \\
\hline & Nov. 27,1946 & 9.70 & 1,420 & 1958 & $\begin{array}{l}\text { Nov. } 6,1957 \\
\text { Nov. } 13,1957\end{array}$ & $\begin{array}{l}12.09 \\
11.88\end{array}$ & $\begin{array}{l}3,600 \\
3,200\end{array}$ \\
\hline \multirow{3}{*}{1948} & $\begin{array}{l}\text { Nov. 23, } 1947 \\
\text { Dec. } 8,1947 \\
\text { Dec. 16, } 1947 \\
\text { Jan. 2, } 1948 \\
\text { Mar. 2, } 1948\end{array}$ & $\begin{array}{r}9.45 \\
9.95 \\
10.29 \\
9.50 \\
10.67\end{array}$ & $\begin{array}{l}1,160 \\
1,750 \\
2,300 \\
1,200 \\
3,250\end{array}$ & & $\begin{array}{l}\text { Jan. } 21,1958 \\
\text { Apr. } 27,1958 \\
\text { May } 1,1958 \\
\text { May } 4,1958\end{array}$ & $\begin{array}{l}11.13 \\
17.80 \\
13.02 \\
10.48\end{array}$ & $\begin{array}{r}1,900 \\
28,900 \\
5,750 \\
1,220\end{array}$ \\
\hline & $\begin{array}{l}\text { Mar. } 23,1948 \\
\text { Apr. 14, } 1948\end{array}$ & $\begin{array}{l}9.30 \\
8.88\end{array}$ & $\begin{array}{l}1,160 \\
1,070\end{array}$ & 1959 & Mar. 6,1959 & 10.26 & 1,030 \\
\hline & May 12,1948 & 10.87 & 3,990 & 1960 & Jan. 14, 1960 & 11.42 & 2,160 \\
\hline 1949 & Jan. 27, 1949 & 10.48 & 3,100 & 1961 & $\begin{array}{l}\text { Dec. 8, } 1960 \\
\text { Dec. 11, } 1960\end{array}$ & $\begin{array}{l}11.35 \\
11.48\end{array}$ & $\begin{array}{l}2,160 \\
2,400\end{array}$ \\
\hline \multirow[t]{5}{*}{1950} & $\begin{array}{l}\text { Oct. } 1949 \\
\text { Oct. 22, } 1949\end{array}$ & $\begin{array}{l}10.02 \\
11.59\end{array}$ & $\begin{array}{l}2,180 \\
6,410\end{array}$ & & Jan. 8, 1961 & 10.35 & 1,050 \\
\hline & Oct. 25,1949 & 11.62 & 6,510 & 1962 & Dec. 17, 1961 & 10.84 & 1,550 \\
\hline & $\begin{array}{l}\text { Jan. } 13,1950 \\
\text { Feb. } 2,1950\end{array}$ & $\begin{array}{l}11.20 \\
11.10\end{array}$ & $\begin{array}{l}5,090 \\
4,770\end{array}$ & & $\begin{array}{l}\text { Jan. } 27,1962 \\
\text { Feb. } 27,1962\end{array}$ & $\begin{array}{l}10.33 \\
11.33\end{array}$ & $\begin{array}{l}1,030 \\
2,160\end{array}$ \\
\hline & Feb. 12,1950 & 12.59 & 10,100 & & Mar. 11, 1962 & 10.53 & 1,230 \\
\hline & & & & 1963 & Apr. 30,1963 & 9.86 & 696 \\
\hline
\end{tabular}

a Annual peak only. 
Location.--Lat $33^{\circ} 08^{\prime} 00^{\prime \prime}$, long $96^{\circ} 04^{\prime} 35^{\prime \prime}$, on right bank at downstream side of bridge on U. S. Highway 67, 0.3 mile downstream from Horse Creek, 0.9 mile downstream from Loulsiana and Arkansas Railway Co. bridge, and 1.8 miles south of Greenville, Hunt County.

Drainage area. $--77.7 \mathrm{sq} \mathrm{mi}$.

Gage.--Recording. Datum of gage is $485.07 \mathrm{ft}$ above mean sea level, datum of 1929.

Stage-discharge relation.--Defined by current-meter measurements below 3,800 cfs and extended above.

Bankfull stage.--12 ft.

Historical data.--Maximum stage since 1895, 22 ft in May 1935, from information by City Engineer of Greenville.

Remarks.--Base for partial-duration series, 1,000 cfs. Rain gage at site.

Peak stages and discharges

\begin{tabular}{|c|c|c|c|c|c|c|c|}
\hline $\begin{array}{l}\text { Water } \\
\text { year }\end{array}$ & Date & $\begin{array}{c}\text { Gage } \\
\text { height } \\
\text { (feet) }\end{array}$ & $\begin{array}{c}\text { Discharge } \\
\text { (cfs) }\end{array}$ & $\begin{array}{r}\text { Water } \\
\text { year }\end{array}$ & Date & $\begin{array}{c}\text { Gage } \\
\text { height } \\
\text { (feet) }\end{array}$ & $\begin{array}{c}\text { Discharge } \\
\text { (cfs) }\end{array}$ \\
\hline 1959 & Oct 4.1059 & 15.67 & - & 1961 & $\begin{array}{l}\text { Mar. 27, } 1961 \\
\text { Mar. 29, } 1961\end{array}$ & $\begin{array}{l}15.42 \\
16.00\end{array}$ & $\begin{array}{l}1,960 \\
3,770\end{array}$ \\
\hline 1960 & $\begin{array}{l}\text { Oct. } 4,1959 \\
\text { Nov. } 4,1959 \\
\text { Dec. } 16,1959 \\
\text { Feb. } 3,1960 \\
\text { Apr. } 30,1960 \\
\text { May } 6,1960 \\
\text { May 26, } 1960 \\
\text { July } 15,1960\end{array}$ & $\begin{array}{l}15.67 \\
15.49 \\
16.68 \\
15.41 \\
15.72 \\
14.49 \\
14.43 \\
15.05\end{array}$ & $\begin{array}{l}2,480 \\
2,000 \\
8,820 \\
1,840 \\
2,640 \\
1,050 \\
1,020 \\
1,400\end{array}$ & 1962 & $\begin{array}{l}\text { Nov. 22, } 1961 \\
\text { Dec. 9, } 1961 \\
\text { Dec. 16, } 1961 \\
\text { Mar. 31, } 1962 \\
\text { Apr. 27, } 1962 \\
\text { May 1, } 1962 \\
\text { Sept. 1, } 1962 \\
\text { Sept. 7, } 1962\end{array}$ & $\begin{array}{l}15.43 \\
15.40 \\
14.99 \\
15.11 \\
14.90 \\
15.35 \\
15.10 \\
15.95\end{array}$ & $\begin{array}{l}1,880 \\
1,820 \\
1,310 \\
1,400 \\
1,240 \\
1,740 \\
1,400 \\
3,540\end{array}$ \\
\hline 1961 & $\begin{array}{l}\text { Oct. } 28,1960 \\
\text { Dec. } 8,1960 \\
\text { Dec. 10, } 1960 \\
\text { Dec. } 31,1960 \\
\text { Jan. } 7,1961 \\
\text { Feb. } 7,1961\end{array}$ & $\begin{array}{l}15.63 \\
15.64 \\
15.68 \\
15.43 \\
15.62 \\
14.66\end{array}$ & $\begin{array}{l}2,360 \\
2,390 \\
2,510 \\
1,980 \\
2,330 \\
1,280\end{array}$ & 1963 & $\begin{array}{l}\text { Nov. } 27,1952 \\
\text { Apr. } 29,1963 \\
\text { May 7, } 1963 \\
\text { May 28, } 1963 \\
\text { July 15, } 1963\end{array}$ & $\begin{array}{l}15.78 \\
15.06 \\
14.53 \\
16.45 \\
15.98\end{array}$ & $\begin{array}{l}2,850 \\
1,360 \\
1,020 \\
6,730 \\
3,680\end{array}$ \\
\hline
\end{tabular}

8-173. South Fork Sabine River near Quinlan, Tex. (1)

Location.--Lat $32^{\circ} 53^{\prime} 52^{\prime \prime}$, long $96^{\circ} 15^{\prime} 11^{\prime \prime}$, on right bank at downstream side of bridge on Farm Road 1565, 2.4 miles upstream from Dry Creek, 6.2 miles upstream from Bearpen Creek, 7 miles southwest of Quinlan, Hunt County, and 25 miles upstream from mouth.

Drainage area. $--78.7 \mathrm{sq} \mathrm{mi}$.

Gage.--Recording. Datum of gage is $461.40 \mathrm{ft}$ above mean sea level, datum of 1929.

Stage-discharge relation.--Defined by current-meter measurements below 5,700 cf's and extended above by contracted-opening measurement of $11,500 \mathrm{cfs}$.

Bankfull stage.--13 ft.

Historical data.--Maximum stage since at least 1890, $21 \mathrm{ft} \mathrm{July} \mathrm{29,} \mathrm{1902,} \mathrm{from} \mathrm{information} \mathrm{by}$ local resident.

Remarks.--Base for partial-duration series, 1,000 cfs. Rain gage at site.

Peak stages and discharges

\begin{tabular}{|c|c|c|c|c|c|c|c|}
\hline $\begin{array}{r}\text { Water } \\
\text { year }\end{array}$ & Date & $\begin{array}{c}\text { Gage } \\
\text { height } \\
\text { (feet) }\end{array}$ & $\begin{array}{c}\text { Discharge } \\
\text { (cfs) }\end{array}$ & $\begin{array}{r}\text { Water } \\
\text { year }\end{array}$ & Date & $\begin{array}{c}\text { Gage } \\
\text { height } \\
\text { (feet) }\end{array}$ & $\begin{array}{c}\text { Discharge } \\
\text { (cfs) }\end{array}$ \\
\hline 1961 & $\begin{array}{l}\text { Oct. } 1,1959 \\
\text { Oct. } 4,1959 \\
\text { Nov. } 4,1959 \\
\text { Dec. } 15,1959 \\
\text { Dec. } 31,1959 \\
\text { Jan. } 5,1960 \\
\text { Feb. } 3,1960 \\
\text { May } \quad 6,1960 \\
\text { Dec. } 8,1960\end{array}$ & $\begin{array}{l}16.48 \\
15.53 \\
14.36 \\
15.77 \\
14.30 \\
15.03 \\
14.79 \\
13.85 \\
14.90\end{array}$ & $\begin{array}{l}4,800 \\
2,820 \\
1,480 \\
3,200 \\
1,440 \\
2,150 \\
1,900 \\
1,080 \\
2,000\end{array}$ & 1962 & $\begin{array}{l}\text { Dec. 10, } 1960 \\
\text { Dec. 31, } 1960 \\
\text { Jan. } 8,1961 \\
\text { Feb. } 7,1961 \\
\text { Mar. } 27,1961 \\
\text { Nov. 21, } 1961 \\
\text { Dec. 9, } 1961 \\
\text { Dec. 16, } 1961 \\
\text { Feb. 26, } 1962\end{array}$ & $\begin{array}{l}15.05 \\
13.84 \\
15.21 \\
14.52 \\
15.65 \\
15.14 \\
15.22 \\
14.62 \\
14.71\end{array}$ & $\begin{array}{l}2,150 \\
1,080 \\
2,320 \\
1,600 \\
3,000 \\
1,600 \\
1,650 \\
1,100 \\
1,170\end{array}$ \\
\hline
\end{tabular}


Peak stages and discharges of South Fork Sabine River near quinlan, Tex.--Continued

\begin{tabular}{|c|c|c|c|c|c|c|c|}
\hline $\begin{array}{r}\text { Water } \\
\text { year }\end{array}$ & Date & $\begin{array}{c}\text { Gage } \\
\text { height } \\
\text { (feet) }\end{array}$ & $\begin{array}{c}\text { Discharge } \\
\text { (cfs) }\end{array}$ & $\begin{array}{l}\text { Water } \\
\text { year }\end{array}$ & Date & $\begin{array}{c}\text { Gage } \\
\text { height } \\
\text { (feet) }\end{array}$ & $\begin{array}{l}\text { Discharge } \\
\text { (cfs) }\end{array}$ \\
\hline 1962 & $\begin{array}{l}\text { Mar. 31, } 1962 \\
\text { Apr. 11, } 1962 \\
\text { Apr. 24, } 1962 \\
\text { Apr. 28, } 1962 \\
\text { May 1, } 1962 \\
\text { June 27, } 1962\end{array}$ & $\begin{array}{l}14.69 \\
14.99 \\
14.92 \\
15.64 \\
14.66 \\
15.27\end{array}$ & $\begin{array}{l}1,170 \\
1,450 \\
1,350 \\
2,280 \\
1,140 \\
1,700\end{array}$ & $\begin{array}{c}1962 \\
1963\end{array}$ & $\begin{array}{l}\text { June 29, } 1962 \\
\text { July 27, } 1962 \\
\text { Sept. 8, } 1962 \\
\text { Oct. 28, } 1962 \\
\text { Nov. 27, } 1962 \\
\text { Apr. 28, } 1963\end{array}$ & $\begin{array}{l}14.70 \\
14.95 \\
15.29 \\
15.21 \\
15.77 \\
16.70\end{array}$ & $\begin{array}{r}1,170 \\
1,400 \\
1,750 \\
2,120 \\
4,200 \\
11,500\end{array}$ \\
\hline
\end{tabular}

8-224. Socagee Creek near. Carthage, Tex. (19)

Location.--Lat $32^{\circ} 13^{\prime} 54^{\prime \prime}$, long $94^{\circ} 05^{\prime} 31^{\prime \prime}$, on right bank at downstream side of bridge on Farm Road 123, 1.4 miles upstream from Salt Creek, 15 miles east of Carthage, Panola County, and 16 miles upstream from mouth.

Drainage area. $--82.6 \mathrm{sq} \mathrm{mi}$.

Gage.--Recording. Datum of gage is $228.3 \mathrm{ft}$ above mean sea level (from Texas Highway Department bridge plans).

Stage-discharge relation.---Defined by current-meter measurements below 800 cfs and extended above by logarithmic plotting.

Bankfull stage. ---10 ft.

Remarks.--Only annual peaks are shown.

Peak stages and discharges

\begin{tabular}{c|c|c|c||c|c|c|c}
\hline $\begin{array}{c}\text { Water } \\
\text { year }\end{array}$ & Date & $\begin{array}{c}\text { Gage } \\
\text { height } \\
\text { (feet) }\end{array}$ & $\begin{array}{c}\text { Discharge } \\
\text { (cfs) }\end{array}$ & $\begin{array}{c}\text { Water } \\
\text { year }\end{array}$ & $\begin{array}{c}\text { Gage } \\
\text { height } \\
\text { (feet) }\end{array}$ & $\begin{array}{c}\text { Discharge } \\
\text { (cfs) }\end{array}$ \\
\hline 1962 & May 2, 1962 & 10.28 & 1,820 & 1963 & Apr. 8, 1963 & 7.07 & 119 \\
\hline
\end{tabular}


8- 232. Tenaha Creek near Shelbyville, Tex.(11)

Location.--Lat 31 $45^{\prime} 56^{\prime \prime}$, long $94^{\circ} 05^{\prime} 02^{\prime \prime}$, near center of span at downstream side of bridge on State Highway 87, 1 mile northwest of Shelbyville, Shelby County, 4.2 miles downstream from Gulf, Colorado and Santa Fe Railway Co. bridge, and 5.0 miles upstream from Beauchamp Creek.

Dralnage area. $--97.8 \mathrm{sq} \mathrm{ml}$.

Gage.--Nonrecording.

Stage-discharge relation.--Defined by current-meter measurements.

Bankful.1 stage. $--9 \mathrm{ft}$.

Historical data.--Flood of Nov. 23, 1940, was highest since 1884, from information by local residents.

Remarks.--Base for partial-duration serles, $800 \mathrm{cfs}$.

\begin{tabular}{|c|c|c|c|c|c|c|c|}
\hline $\begin{array}{l}\text { Water } \\
\text { year }\end{array}$ & Date & $\begin{array}{c}\text { Gage } \\
\text { helght } \\
\text { (feet) }\end{array}$ & $\begin{array}{c}\text { D1scharge } \\
\text { (cfs) }\end{array}$ & $\begin{array}{l}\text { Water } \\
\text { year }\end{array}$ & Date & $\begin{array}{c}\text { Gage } \\
\text { helght } \\
\text { (feet) }\end{array}$ & $\begin{array}{c}\text { Discharge } \\
(\text { cfs })\end{array}$ \\
\hline 2941 & Nov. 23,1940 & 15 & - & 1958 & Nov. 23,1957 & 9.85 & 1,440 \\
\hline 1952 & $\begin{array}{l}\text { Mar. 11, } 1952 \\
\text { Apr. 13, } 1952 \\
\text { Apr. 24, } 1952\end{array}$ & $\begin{array}{r}9.70 \\
9.79 \\
10.10\end{array}$ & $\begin{array}{r}1,090 \\
1,700 \\
22,180\end{array}$ & & $\begin{array}{ll}\text { Jan. } 21, & 1958 \\
\text { May 4, } 1958 \\
\text { June } 17,1958\end{array}$ & $\begin{array}{r}7.91 \\
10.05 \\
10.80 \\
9.40\end{array}$ & $\begin{array}{r}1,700 \\
1,800 \\
3,360 \\
880\end{array}$ \\
\hline 1953 & $\begin{array}{l}\text { Mar.11, } 1953 \\
\text { Mar. 15, } 1953\end{array}$ & $\begin{array}{r}13.85 \\
9.80\end{array}$ & $\begin{array}{r}15,200 \\
1,700\end{array}$ & 1959 & Apr. 19, 1959 & 10.44 & 2,600 \\
\hline & $\begin{array}{l}\text { Apr. } 29,1.953 \\
\text { May } 4,1953\end{array}$ & $\begin{array}{l}13.63 \\
12.42\end{array}$ & $\begin{array}{r}13,900 \\
8,110\end{array}$ & 1960 & $\begin{array}{l}\text { Nov. } 6,1959 \\
\text { Dec. 17, } 1959\end{array}$ & $\begin{array}{l}10.25 \\
10.90\end{array}$ & $\begin{array}{l}1,140 \\
2,700\end{array}$ \\
\hline & $\begin{array}{lll}\text { May } & 12,1953 \\
\text { May } 17, & 1953\end{array}$ & $\begin{array}{l}13.00 \\
12.00\end{array}$ & $\begin{array}{r}10,500 \\
6,600\end{array}$ & & Feb. 25,1960 & 10.30 & 1,200 \\
\hline 1954 & May 12, 1954 & 10.00 & 2,020 & 1961 & $\begin{array}{l}\text { Nov. } 23,1960 \\
\text { Dec. 9, } 1960 \\
\text { Jan. } 8,1961\end{array}$ & $\begin{array}{l}11.30 \\
11.57 \\
12.20\end{array}$ & $\begin{array}{l}2,970 \\
3,560 \\
5,550\end{array}$ \\
\hline 1955 & $\begin{array}{l}\text { Mar. 23, } 1955 \\
\text { Apr. 10, } 1955 \\
\text { Apr. 13, } 1955\end{array}$ & $\begin{array}{r}9.03 \\
9.40 \\
10.20\end{array}$ & $\begin{array}{r}889 \\
1,090 \\
2,340\end{array}$ & & $\begin{array}{l}\text { Jan. } 13,1961 \\
\text { Jan. } 25,1961 \\
\text { Mar. 17, } 1961 \\
\text { Mar. 31, } 1961\end{array}$ & $\begin{array}{l}10.17 \\
10.25 \\
13.33 \\
10.00\end{array}$ & $\begin{array}{r}1,200 \\
1,300 \\
10,900 \\
1,050\end{array}$ \\
\hline 1956 & Apr. 6, 1956 & 9.39 & 1,080 & & Sept.14, 1961 & 10.54 & 1,660 \\
\hline 1957 & $\begin{array}{l}\text { Apr. 4, } 1957 \\
\text { Apr. 25, } 1957 \\
\text { May 1, } 1957 \\
\text { June 3, } 1957 \\
\text { June 23, } 1957\end{array}$ & $\begin{array}{r}9.40 \\
10.20 \\
10.52 \\
9.50 \\
9.80\end{array}$ & $\begin{array}{r}880 \\
2,500 \\
3,040 \\
1,030 \\
1,700\end{array}$ & 1962 & $\begin{array}{l}\text { Dec. } 10,1961 \\
\text { Dec. 12, } 1961 \\
\text { Dec. 15, } 1961 \\
\text { Dec. 18, } 1961 \\
\text { Apr. 12, } 1962 \\
\text { Apr. 28, } 1962\end{array}$ & $\begin{array}{r}12.30 \\
10.88 \\
9.80 \\
10.41 \\
9.75 \\
10.38\end{array}$ & $\begin{array}{r}5,900 \\
2,320 \\
960 \\
1,600 \\
930 \\
1,600\end{array}$ \\
\hline 1.958 & $\begin{array}{l}\text { Oct. } 23,1957 \\
\text { Nov. } 8,1957 \\
\text { Nov. } 18,1957\end{array}$ & $\begin{array}{r}9.63 \\
11.20 \\
9.40\end{array}$ & $\begin{array}{r}1,110 \\
4,240 \\
880\end{array}$ & 1963 & $\begin{array}{l}\text { May } 1,1962 \\
\text { Apr. 6, } 1963\end{array}$ & $\begin{array}{r}10.46 \\
9.50\end{array}$ & $\begin{array}{r}1,660 \\
760\end{array}$ \\
\hline
\end{tabular}

a Maximum Mar. 6 to Sept. 30; probably maximum for year. 
8-300. Cypress Creek near Buna, Tex. (20)

Location.--Lat $30^{\circ} 25^{\prime} 45^{\prime \prime}$, long $93^{\circ} 54^{\prime} 20^{\prime \prime}$, near center of span at downstream side of bridge on State Farm Road $253,1.0$ mile downstream from unnamed tributary, 3.2 miles east of Buna, Jasper County, and 10 miles upstream from Little Cypress Creek.

Dra1nage area. $--69.2 \mathrm{sq} \mathrm{ml}$.

Gage.--Nonrecording prior to oct. 23, 1957; recording thereafter. Datum of gage is $46 \mathrm{ft}$ above mean sea level (State Highway Department bridge plans).

Stage-discharge relation.--Defined by current-meter measurements.

Bankfull stage. $-9 \mathrm{ft}$.

Remarks.--Base for partial-duration series, 1,000 cfs.

\begin{tabular}{|c|c|c|c|c|c|c|c|}
\hline $\begin{array}{l}\text { Water } \\
\text { year }\end{array}$ & Date & $\begin{array}{c}\text { aage } \\
\text { he1ght } \\
\text { (feet) }\end{array}$ & $\begin{array}{c}\text { Discharge } \\
\text { (cfs) }\end{array}$ & $\begin{array}{l}\text { Water } \\
\text { year }\end{array}$ & Date & $\begin{array}{l}\text { Gage } \\
\text { he1ght } \\
\text { (feet) }\end{array}$ & $\begin{array}{c}\text { D1scharge } \\
\text { (cfs) }\end{array}$ \\
\hline 1952 & 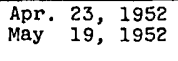 & $\begin{array}{l}11.93 \\
11.12\end{array}$ & $\begin{array}{r}a 3,800 \\
2,500\end{array}$ & 1957 & $\begin{array}{l}\text { June } 28,1957 \\
\text { Sept.26, } 1957\end{array}$ & $\begin{array}{l}10.40 \\
11.00\end{array}$ & $\begin{array}{l}1,620 \\
2,350\end{array}$ \\
\hline 1953 & $\begin{array}{l}\text { Feb. } 24,1953 \\
\text { Apr. } 30,1953 \\
\text { May } 19,1953 \\
\text { Apr. } 15,1954\end{array}$ & $\begin{array}{l}10.00 \\
11.65 \\
11.14 \\
11.02\end{array}$ & $\begin{array}{l}1,220 \\
3,320 \\
2,560 \\
2,350\end{array}$ & 1958 & $\begin{array}{l}\text { Nov. } 14,1957 \\
\text { Nov. } 22,1957 \\
\text { Feb. 23, } 1958 \\
\text { Sept.22, } 1958\end{array}$ & $\begin{array}{r}10.48 \\
10.40 \\
9.95 \\
10.95\end{array}$ & $\begin{array}{l}1,730 \\
1,620 \\
1,180 \\
2,420\end{array}$ \\
\hline dout & 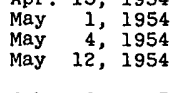 & $\begin{array}{l}10.30 \\
10.35 \\
10.81\end{array}$ & $\begin{array}{l}1,520 \\
1,570 \\
2,080\end{array}$ & 1959 & $\begin{array}{l}\text { Jan. } 30,1959 \\
\text { Feb. } 2,1959 \\
\text { Feb. } 25,1959 \\
\text { Apr. } 12,1959\end{array}$ & $\begin{array}{r}10.82 \\
10.50 \\
9.81 \\
9.78\end{array}$ & $\begin{array}{l}2,080 \\
1,730 \\
1,100 \\
1,100\end{array}$ \\
\hline 2955 & $\begin{array}{l}\text { Feb. } 6,1955 \\
\text { Apr. } 11,1955 \\
\text { Apr. } 13,1955\end{array}$ & $\begin{array}{r}11.13 \\
9.90 \\
11.95\end{array}$ & $\begin{array}{l}2,580 \\
1,130 \\
3,800\end{array}$ & 1960 & $\begin{array}{l}\text { July 26, } 1959 \\
\text { Dec. } 17,1959\end{array}$ & $\begin{array}{l}10.68 \\
10.84\end{array}$ & 2,140 \\
\hline 1956 & Feb. 4; 1956 & 9.73 & 1,020 & 1961 & $\begin{array}{l}\text { Dec. } 31,1960 \\
\text { Jan. } 8,1961\end{array}$ & $\begin{array}{l}10.77 \\
11.36\end{array}$ & $\begin{array}{l}2,020 \\
2,880\end{array}$ \\
\hline 1957 & $\begin{array}{l}\text { Dec. } 22,1956 \\
\text { Mar. } 18,1957 \\
\text { May } 2,1957\end{array}$ & $\begin{array}{l}10.30 \\
10.55 \\
10.15\end{array}$ & $\begin{array}{l}1,520 \\
1,780 \\
1,370\end{array}$ & $\begin{array}{l}1962 \\
1963\end{array}$ & $\begin{array}{l}\text { Feb. 18, } 1961 \\
\text { Sept.14, } 1961 \\
\text { Dec. } 18,1961 \\
\text { Sept.18, } 1963\end{array}$ & $\begin{array}{l}11.01 \\
10.06 \\
10.14 \\
13.28\end{array}$ & $\begin{array}{l}2,000 \\
2,350 \\
1,210 \\
1,290 \\
7,100 \\
\end{array}$ \\
\hline
\end{tabular}

8-310. Cow Bayou near Maurtceville, Tex.(20)

Location.--Lat $30^{\circ} 11^{\prime} 05^{\prime \prime}$, long $93^{\circ} 54^{\prime} 40^{\prime \prime}$, near center of span at downstream slde of bridge on State H1ghway 12, half a mile upstream from Kansas C1ty Southern Raliway Co. bridge, and 3 miles southwest of Mauriceville, Orange County.

Drainage area. $--83.3 \mathrm{sq} \mathrm{mi}$.

Gage.--Nonrecording prior to Oct. 23, 1957; recording thereafter. Datum of gage Is $4.7 \mathrm{ft}$ above mean sea levei (State Highway Department bridge plans).

Stage-discharge relation.--Defined by current-meter measurements; subject to changes owing to channel shifting and backwater from railroad bridge downstream and from local runoff.

Bankfull stage. $--12 \mathrm{ft}$.

Historlcal data.--Floods of Feb. 2, 1952, and Sept. 23, 1958, were highest since at least 1940, from information by State Highway Department.

Remarks.--Base for partial-duration series, $900 \mathrm{cfs}$.

\begin{tabular}{|c|c|c|c|c|c|c|c|}
\hline $\begin{array}{l}\text { Water } \\
\text { year }\end{array}$ & Date & $\begin{array}{c}\text { Gage } \\
\text { helght } \\
\text { (feet) }\end{array}$ & $\begin{array}{c}\text { Discharge } \\
\text { (cfs) }\end{array}$ & $\begin{array}{l}\text { Water } \\
\text { year }\end{array}$ & Date & $\begin{array}{c}\text { Gage } \\
\text { he1ght } \\
\text { (feet) }\end{array}$ & $\begin{array}{c}\text { Discharge } \\
\text { (cfs) }\end{array}$ \\
\hline 1952 & $\begin{array}{l}\text { Feb. 2, } 1952 \\
\text { Apr. } 24,1952\end{array}$ & $\begin{array}{l}16.5 \\
15.16\end{array}$ & a3, 380 & 1958 & $\begin{array}{l}\text { Feb. } 27,1958 \\
\text { Sept. } 23,1958\end{array}$ & $\begin{array}{l}10.78 \\
16.71\end{array}$ & $\begin{array}{r}925 \\
4,300\end{array}$ \\
\hline 1953 & May 19,1953 & 14.15 & 1,950 & 1959 & Feb. 4, 1959 & $\begin{array}{l}14.68 \\
11.97\end{array}$ & 2,440 \\
\hline 1954 & Apr. 19, 1954 & 8.70 & 659 & & $\begin{array}{l}\text { Feb. } 25,1959 \\
\text { Apr. } 12,1959\end{array}$ & $\begin{array}{l}12.16 \\
12.97\end{array}$ & $\begin{array}{l}1,180 \\
1,390\end{array}$ \\
\hline 1955 & $\begin{array}{l}\text { Feb. 9, } 1955 \\
\text { Apr. 15, } 1955\end{array}$ & $\begin{array}{l}10.72 \\
10.70\end{array}$ & $\begin{array}{l}962 \\
928\end{array}$ & 1960 & $\begin{array}{l}\text { July } 27,1959 \\
\text { Feb, } 21,1960\end{array}$ & $\begin{array}{r}14.17 \\
8.47\end{array}$ & $\begin{array}{r}1,970 \\
617\end{array}$ \\
\hline 1956 & Feb. 9,1956 & 11.20 & 1,050 & 1961 & Tan 21961 & 1301 & 1,370 \\
\hline 1957 & $\begin{array}{l}\text { Dec. } 24,1956 \\
\text { Mar. } 21,1957 \\
\text { May } 2,1957 \\
\text { June } 30,1957\end{array}$ & $\begin{array}{r}\text { b13.80 } \\
12.30 \\
\text { b12.00 } \\
12.21\end{array}$ & $\begin{array}{l}1,700 \\
1,290 \\
1,160 \\
1,260\end{array}$ & & $\begin{array}{ll}\text { Jan. } 9, & 1961 \\
\text { Feb. } 19, & 1961 \\
\text { June } 19, & 1961 \\
\text { July } 12, & 1961\end{array}$ & $\begin{array}{l}15.01 \\
14.56 \\
11.48 \\
11.96\end{array}$ & $\begin{array}{l}1,900 \\
1,980 \\
1,040 \\
1,140\end{array}$ \\
\hline 1958 & Nov. $26,1957$. & 11.84 & 1,100 & $\begin{array}{l}1962 . \\
1963\end{array}$ & $\begin{array}{l}\text { Dec. 17-20, } \\
\quad 1961 \\
\text { Sept.19, } 1963\end{array}$ & $\begin{array}{l}10.64 \\
18.15\end{array}$ & $\begin{array}{r}895 \\
4,600\end{array}$ \\
\hline
\end{tabular}

a Maximum for Mar. 10 to Sept 


\section{8-311.45. Unnamed tributary (watershed 3) of Prairie Creek near Tyler, Tex.(10)}

Location.--Lat $32^{\circ} 28^{\prime}$, long $95^{\circ} 25^{\prime}, 10$ miles northwest of Tyler, Smith County.

Drainage area. $--0.0124 \mathrm{sq} \mathrm{ml}$.

Gage.--Recording.

Remarks.--Records furnished by U.S. Department of Agriculture, Agricultural Research Service. Only: annual (calendar year) peaks are shown.

Peak stages and discharges

\begin{tabular}{|c|c|c|c|c|c|c|c|}
\hline $\begin{array}{l}\text { Calen- } \\
\text { dar } \\
\text { year }\end{array}$ & Date & $\begin{array}{c}\text { Gage } \\
\text { helght } \\
\text { (reet) }\end{array}$ & $\begin{array}{c}\text { Discharge } \\
(\mathrm{cfs})\end{array}$ & $\begin{array}{l}\text { Calen- } \\
\text { dar } \\
\text { year }\end{array}$ & Date & $\begin{array}{c}\text { Gage } \\
\text { helght } \\
\text { (feet) }\end{array}$ & $\begin{array}{c}\text { Discharge } \\
(\mathrm{cfs})\end{array}$ \\
\hline $\begin{array}{l}1933 \\
1934 \\
1935\end{array}$ & $\begin{array}{lr}\text { July } & 19,1933 \\
\text { Apr. } & 5,1934 \\
\text { May } & 5,1935\end{array}$ & $\overline{-}$ & $\begin{array}{l}1.3 \\
1.1 \\
2.0\end{array}$ & $\begin{array}{l}1938 \\
1939 \\
1940\end{array}$ & $\begin{array}{l}\text { Jan. } 23,1938 \\
\text { Feb. } 25,1939 \\
\text { Nov. } 23,1940\end{array}$ & $\begin{array}{l}- \\
-\end{array}$ & $\begin{array}{r}3.2 \\
.1 \\
.5\end{array}$ \\
\hline $\begin{array}{l}1936 \\
1937 \\
\end{array}$ & $\begin{array}{l}\text { May } 9,1936 \\
\text { Nov. } 15,1937\end{array}$ & - & $\begin{array}{l}8.1 \\
1.1\end{array}$ & 1941 & June 7,1941 & - & .6 \\
\hline
\end{tabular}

\section{8-311.50. Unnamed tributary (watershed 4) of Prairie Creek near Tyler, Tex. (10)}

Location.--Lat $32^{\circ} 28^{\prime}$, long $95^{\circ} 25^{\prime}, 10$ miles northwest of Tyler, Smith County. Drainage area $--0.0095 \mathrm{sq} \mathrm{m} 1 ; 0.0100 \mathrm{sq} \mathrm{ml} 1931$ to Dec. 31, $1932 ; 0.0087 \mathrm{sq} \mathrm{m} 1$ Jan. I, 1933, to March 1939.

Gage.--Recording.

Remarks.--Records furnished by U.S. Department of Agriculture, Agricultural Research Service. Only annual (calendar year) peaks are shown.

Peak stages and discharges

\begin{tabular}{|c|c|c|c|c|c|c|c|}
\hline $\begin{array}{l}\text { Calen- } \\
\text { dar } \\
\text { year }\end{array}$ & Date & $\begin{array}{c}\text { Cage } \\
\text { height } \\
\text { (feet) }\end{array}$ & $\begin{array}{c}\text { Discharge } \\
(\text { cfs })\end{array}$ & $\begin{array}{l}\text { Calen- } \\
\text { dar } \\
\text { year }\end{array}$ & Date & $\begin{array}{l}\text { Gage } \\
\text { helght } \\
\text { (feet) }\end{array}$ & $\begin{array}{c}\text { Discharge } \\
\text { (cfs) }\end{array}$ \\
\hline $\begin{array}{l}1931 \\
1932 \\
1933 \\
1934 \\
1935 \\
1936 \\
\end{array}$ & $\begin{array}{l}\text { June } 16,1931 \\
\text { Dec. } 23,1932 \\
\text { Mar. } 30,1933 \\
\text { Mar. } 25,1934 \\
\text { July } 3,1935 \\
\quad \text { May } 1936\end{array}$ & $\begin{array}{l}- \\
- \\
- \\
- \\
-\end{array}$ & $\begin{array}{l}19 \\
17 \\
25 \\
14 \\
22 \\
\text { (a) }\end{array}$ & $\begin{array}{l}1937 \\
1938 \\
1939 \\
1940 \\
1941\end{array}$ & $\begin{array}{lr}\text { Nov. 15, } & 1937 \\
\text { Mar. 28, } & 1938 \\
\text { July } 9, & 1939 \\
\text { June } 28, & 1940 \\
\text { July 11, } & 1941\end{array}$ & $\begin{array}{l}- \\
- \\
- \\
-\end{array}$ & $\begin{array}{l}26 \\
20 \\
28 \\
31 \\
32\end{array}$ \\
\hline
\end{tabular}

a No record for peak-producing storm of May 1936.

8-311.55. Unnamed tributary (watershed 5) of Prairie Creek near Tyler, Tex.(10)

Location.--Lat $32^{\circ} 28^{\prime}$, long $95^{\circ} 25^{\prime}, 10$ miles northwest of Tyler, Smith County. Drainage area. $--0.0025 \mathrm{sq} \mathrm{ml}$.

Gage.--Recording.

Remarks.--Records furnished by U.S. Department of Agriculture, Agricultural Research Service. Only annual (calendar year) peaks are shown.

\begin{tabular}{|c|c|c|c|c|c|c|c|}
\hline $\begin{array}{l}\text { Calen- } \\
\text { dar } \\
\text { year }\end{array}$ & Date & $\begin{array}{c}\text { Gage } \\
\text { helght } \\
\text { (feet) }\end{array}$ & $\begin{array}{c}\text { Discharge } \\
\text { (cfs) }\end{array}$ & $\begin{array}{l}\text { Calen- } \\
\text { dar } \\
\text { year }\end{array}$ & Date & $\begin{array}{c}\text { Gage } \\
\text { nelght } \\
\text { (feet) }\end{array}$ & $\begin{array}{c}\text { D1scharge } \\
\text { (cfs) }\end{array}$ \\
\hline $\begin{array}{l}1933 \\
1934 \\
1935\end{array}$ & $\begin{array}{lrr}\text { Mar. } & 30,1933 \\
\text { Mar. } & 25, & 1934 \\
\text { May } & 3, & 1935\end{array}$ & $\bar{z}$ & $\begin{array}{l}3.9 \\
1.4 \\
3.5\end{array}$ & $\begin{array}{l}1938 \\
1939 \\
1940\end{array}$ & $\begin{array}{ll}\text { Jan. } 23,1938 \\
\text { July } 9,1939 \\
\text { June 28, } 1940\end{array}$ & $\overline{-}$ & $\begin{array}{l}3.3 \\
1.8 \\
3.4\end{array}$ \\
\hline $\begin{array}{l}1936 \\
1937\end{array}$ & $\begin{array}{l}\text { May } \\
\text { Apr. }\end{array}$ & - & $\frac{11}{2.9}$ & 1941 & June 7,1941 & - & 3.5 \\
\hline
\end{tabular}


Location.--Lat $31^{\circ} 08^{\prime} 30^{\prime \prime}$, Long $95^{\circ} 05^{\prime} 10^{\prime \prime}$, on left bank at downstream side of bridge on State Highway 94, 4 miles upstream from Caney Creek, and $6 \frac{1}{2}$ miles northeast of Groveton, Trinity County.

Drainage area. $--79.0 \mathrm{sq} \mathrm{mi}$.

Gage.--Recording. Datum of gage is $251.40 \mathrm{ft}$ above mean sea level, datum of 1929.

Stage-discharge relation.---Defined by current-meter measurements.

Bankfull stage. $--10 \mathrm{ft}$.

Historical da.ta.--Maximum stage since at least 1921, $17 \mathrm{ft}$ in May 1942, from information by local resident.

Remarks.--Base for partial-duration series, $400 \mathrm{cfs}$.

\begin{tabular}{|c|c|c|c|c|c|c|c|}
\hline $\begin{array}{r}\text { Water } \\
\text { year }\end{array}$ & Date & $\begin{array}{l}\text { Gage } \\
\text { height } \\
\text { (feet) }\end{array}$ & $\begin{array}{c}\text { Discharge } \\
\text { (cfs) }\end{array}$ & $\begin{array}{r}\text { Water } \\
\text { year }\end{array}$ & Date & $\begin{array}{c}\text { Gage } \\
\text { height } \\
\text { (feet) }\end{array}$ & $\begin{array}{c}\text { Discharge } \\
\text { (cfs) }\end{array}$ \\
\hline 1962 & $\begin{array}{l}\text { Dec. 10, } 1961 \\
\text { Dec. 18, } 1961 \\
\text { Jan. 27, } 1962 \\
\text { May 1, } 1962\end{array}$ & $\begin{array}{l}11.07 \\
10.74 \\
12.05 \\
13.20\end{array}$ & $\begin{array}{r}466 \\
416 \\
655 \\
1,540\end{array}$ & 1963 & $\begin{array}{l}\text { Dec. 29, } 1962 \\
\text { Feb. 19, } 1963 \\
\text { Apr. 7, } 1963\end{array}$ & $\begin{array}{l}10.57 \\
10.94 \\
11.42\end{array}$ & $\begin{array}{l}440 \\
480 \\
570\end{array}$ \\
\hline
\end{tabular}

\section{8-375. Arenoso Creek near San Augustine, Tex. (11)}

Location.--Lat $31^{\circ} 36^{\prime}$, long $94^{\circ} 17^{\prime}$, at Camp Worth, $\frac{1}{2}$ mile downstream from Nacogdoches and Southeastern Railroad bridge, $4 \frac{1}{2}$ miles upstream from Attoyac Bayou, and 11 miles northwest of San Augustine, San Augustine County.

Drainage area. -- $75.3 \mathrm{sq} \mathrm{mi}$.

Gage.--Nonrecording.

Remarks.--Only annual peaks are shown.

\begin{tabular}{c|c|c|c||c|c|c|c}
\hline $\begin{array}{c}\text { Water } \\
\text { year }\end{array}$ & Date & $\begin{array}{c}\text { Gage } \\
\text { height } \\
\text { (feet) }\end{array}$ & $\begin{array}{c}\text { Discharge } \\
\text { (cfs) }\end{array}$ & $\begin{array}{c}\text { Water } \\
\text { year }\end{array}$ & Date & $\begin{array}{c}\text { Gage } \\
\text { neight } \\
\text { (feet) }\end{array}$ & $\begin{array}{c}\text { Discharge } \\
\text { (cfs) }\end{array}$ \\
\hline 1939 & Feb. 2, 1939 & 9.94 & 834 & 1940 & Dec. 23, 1939 & 12.12 & (a) \\
\hline
\end{tabular}

a Not determined.

8-391. Ayish Bayou near San Augustine, Tex. (11)

Location.--Lat $31^{\circ} 23^{\prime} 46^{\prime \prime}$, long $94^{\circ} 09^{\prime} 03^{\prime \prime}$, near center of span at downstream side of pier of bridge on State Highway 103, 3.0 miles upstream from Turkey Creek, and $9 \frac{1}{2}$ miles south of San Augustine, San Augustine County.

Drainage area. $--89.0 \mathrm{sq} \mathrm{mi}$.

Gage.-Recording. Datum of gage is $190.22 \mathrm{ft}$ above mean sea level, datum of 1929. Prior to June 2, 1959, wire-weight gage at same site and datum.

Stage-discharge relation.--Defined by current-meter measurements below 3,500 cfs and extended above by logarithmic plotting.

Bankfull stage.--ll $\mathrm{ft}$.

Remarks.--Base for partial-duration series, $900 \mathrm{cfs}$. Rain gage at site.

\begin{tabular}{|c|c|c|c|c|c|c|c|}
\hline $\begin{array}{r}\text { Water } \\
\text { year }\end{array}$ & Date & $\begin{array}{c}\text { Gage } \\
\text { height } \\
\text { (feet) }\end{array}$ & $\begin{array}{l}\text { Discharge } \\
(\mathrm{cfs})\end{array}$ & $\begin{array}{l}\text { Water } \\
\text { year }\end{array}$ & Date & $\begin{array}{c}\text { Gage } \\
\text { height } \\
\text { (feet) }\end{array}$ & $\begin{array}{c}\text { Discharge } \\
\text { (cfs) }\end{array}$ \\
\hline $\begin{array}{r}1959 \\
1960\end{array}$ & $\begin{array}{l}\text { Apr. 18, } 1959 \\
\text { July } 26,1959 \\
\text { Dec. } 17 \text { or } 18, \\
1959 \\
\text { Feb. } 24,1960\end{array}$ & $\begin{array}{l}14.50 \\
13.07 \\
12.34 \\
12.77\end{array}$ & $\begin{array}{l}7,750 \\
3,200 \\
2,020 \\
2,620\end{array}$ & 1961 & $\begin{array}{l}\text { Feb. } 17,1961 \\
\text { Mar. } 17,1961 \\
\text { Mar. } 28,1961 \\
\text { Mar. 31, } 1961 \\
\text { Sept.13, } 1961\end{array}$ & $\begin{array}{l}11.93 \\
13.95 \\
11.18 \\
12.77 \\
12.51\end{array}$ & $\begin{array}{r}1,520 \\
5,620 \\
970 \\
2,620 \\
2,250\end{array}$ \\
\hline 2961 & $\begin{array}{l}\text { Nov. } 21,1960 \\
\text { Dec. } 9,1960 \\
\text { Jan. } 8,1961 \\
\text { Jan. } 13,1961\end{array}$ & $\begin{array}{l}11.64 \\
12.46 \\
13.21 \\
12.27\end{array}$ & $\begin{array}{l}1,260 \\
2,180 \\
3,500 \\
1,890\end{array}$ & 1963 & $\begin{array}{l}\text { Dec. 10, } 1961 \\
\text { Dec. 18, } 1961 \\
\text { May 1, } 1962 \\
\begin{array}{l}\text { Dec. 29, } 1962 \\
\text { Apr. 6, } 1963\end{array}\end{array}$ & $\begin{array}{l}13.20 \\
12.30 \\
12.89 \\
11.58 \\
11.54 \\
\end{array}$ & $\begin{array}{l}3,200 \\
1,700 \\
2,900 \\
1,220 \\
1,190\end{array}$ \\
\hline
\end{tabular}


Location.--Lat $33^{\circ} 17^{\prime}$, long $98^{\circ} 18^{\prime}$, on left bank at downstream side of bridge on U.S. H1ghway $281,1.5$ miles upstream from Henderson Creek, 9.3 miles northwest of Jacksboro, Jack County, and 14 miles upstream from mouth.

Drainage area. $--21.6 \mathrm{sq} \mathrm{ml}$.

Gage:--Recording. Datum of gage is $1,016.33 \mathrm{ft}$ above mean sea level (State Highway Department bench mark):

Stage-discharge relation.--Defined by current-meter measurements.

Bankfull stage. $--15 \mathrm{ft}$.

H1storlcal data.--Flood of Apr. 28, 1957, was the highest since at least 1915 from information by local resident.

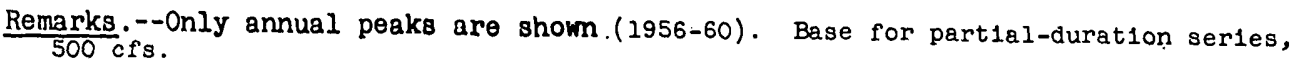

Peak stages and discharges

\begin{tabular}{|c|c|c|c|c|c|c|c|}
\hline $\begin{array}{l}\text { Water } \\
\text { year }\end{array}$ & Date & $\begin{array}{c}\text { agge } \\
\text { helght } \\
\text { (feet) }\end{array}$ & $\begin{array}{c}\text { D1scharge } \\
\text { (cfs) }\end{array}$ & $\begin{array}{l}\text { Water } \\
\text { year }\end{array}$ & Date & $\begin{array}{c}\text { Gage } \\
\text { helght } \\
\text { (feet) }\end{array}$ & $\begin{array}{c}\text { D1gcharge } \\
(\mathrm{cfs})\end{array}$ \\
\hline $\begin{array}{l}1956 \\
1957 \\
1958 \\
1959 \\
1960 \\
1961\end{array}$ & 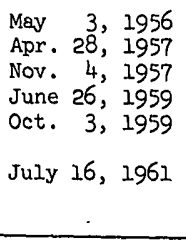 & $\begin{array}{l}21.58 \\
24.45 \\
12.56 \\
14.45 \\
19.65 \\
15.23\end{array}$ & $\begin{array}{l}5,700 \\
6,990 \\
1,760 \\
2,500 \\
4,830 \\
2,840\end{array}$ & 1962 & $\begin{array}{l}\text { Oct. } 2,1961 \\
\text { June 1, } 1962 \\
\text { June 10, } 1962 \\
\text { July 16, } 1962 \\
\text { July 27, } 1962 \\
\text { Sept. 7, } 1962 \\
\begin{array}{l}\text { Nov. 26, } 1962 \\
\text { Apr. 28, } 1963\end{array}\end{array}$ & $\begin{array}{r}9.57 \\
10.27 \\
18.10 \\
8.92 \\
11.13 \\
10.67 \\
\\
9.11 \\
11.55 \\
\end{array}$ & $\begin{array}{r}731 \\
941 \\
4,130 \\
536 \\
1,230 \\
1,060 \\
\\
593 \\
1,370 \\
\end{array}$ \\
\hline
\end{tabular}

8-485. Marine Creek at Fort Worth, Tex.(2)

Location.--Lat $32^{\circ} 48^{\prime} 16^{\prime \prime}$, long $97^{\circ} 21^{\prime} 48^{\prime \prime}$, on left bank at downstream side of bridge on Northwest 33d Street in Fort Worth, Tarrant County, 1.5 miles upstream from North Main street, 2.2 miles upstream from St. Louls Southwestern Rallway bridge, and 2.4 miles upstream from mouth.

Drainage area. $--16.8 \mathrm{sq} \mathrm{mi}$.

Gage.--Recording. Datum of gage is $562.60 \mathrm{ft}$ above mean sea level, datum of I929.

Stage-discharge relation.--Defined by current-meter measurements below 2,700 $\mathrm{cf}$ 's and by slope-area measurement at $24,400 \mathrm{cfs}$.

H1storical data.--Fiood of Apr. 20, 1942, 1s the highest since at least 1907. large floods also occurred in 1908 and 1922 (stages not known), from information by local resident.

Remarks.--Flow from $3.7 \mathrm{sq} \mathrm{ml} \mathrm{partly} \mathrm{regulated} \mathrm{after} \mathrm{Feb.} \mathrm{7,} \mathrm{1957,} \mathrm{by} \mathrm{Cement}$ Creek Reservolr (total capacity, 3,950 acre-ft). Flow from 9.8 sq m1 regulated by flxed outlet after Apr. 17, 1958, at Marine Creek Reservoir (total capacity, 15,370 acre-ft). Base for partial-duration series, $230 \mathrm{cfs}$.

Peak stages and discharges

\begin{tabular}{|c|c|c|c|c|c|c|c|}
\hline $\begin{array}{l}\text { Water } \\
\text { year }\end{array}$ & Date & $\begin{array}{c}\text { Gage } \\
\text { helght } \\
\text { (feet) }\end{array}$ & $\begin{array}{c}\text { D1scharge } \\
\text { (cfs) }\end{array}$ & $\begin{array}{l}\text { Water } \\
\text { year }\end{array}$ & Date & $\begin{array}{c}\text { Gage } \\
\text { helght } \\
\text { (feet) }\end{array}$ & $\begin{array}{c}\text { D1scharge } \\
\text { (cfs) }\end{array}$ \\
\hline 1942 & Apr. 20,1942 & 16.1 & 24,400 & 1956 & Apr. 14,1956 & 2.63 & 438 \\
\hline 1950 & Sept.19, 1950 & 1.48 & a51 & & 1500 &. \pm 0 & 000 \\
\hline 1951 & June 16, 1951 & 2.29 & 266 & 1957 & $\begin{array}{l}\text { Apr. } 19,1951 \\
\text { Apr. } 20,1957 \\
\text { Apr. } 23,1957\end{array}$ & $\begin{array}{l}2.65 \\
4.30 \\
3.50\end{array}$ & $\begin{array}{r}242 \\
1,610 \\
756\end{array}$ \\
\hline 1952 & Apr. 22, 1952 & 1.38 & 37 & & $\begin{array}{l}\mathrm{Apr} .26,1957 \\
\mathrm{May}\end{array}$ & $\begin{array}{l}4.81 \\
3.38\end{array}$ & $\begin{array}{r}2,420 \\
660\end{array}$ \\
\hline 1953 & $\begin{array}{ll}\text { Apr. } 23, & 1953 \\
\text { Apr. } 28,1953\end{array}$ & $\begin{array}{l}2.37 \\
2.31\end{array}$ & $\begin{array}{l}305 \\
275\end{array}$ & & $\begin{array}{l}\text { May } \\
\text { May } \\
\text { May } \\
\text { May, } 1957 \\
30,1957\end{array}$ & $\begin{array}{l}5.47 \\
5.55 \\
3.37\end{array}$ & $\begin{array}{l}4,300 \\
4,600 \\
1,620\end{array}$ \\
\hline 1954 & $\begin{array}{ll}\text { Oct. } 23,1953 \\
\text { Oct. } 25,1953\end{array}$ & $\begin{array}{l}2.84 \\
2.34\end{array}$ & $\begin{array}{l}572 \\
290\end{array}$ & & $\begin{array}{l}\text { June } 2,1957 \\
\text { June } 5,1957\end{array}$ & $\begin{array}{l}4.24 \\
3.58\end{array}$ & $\begin{array}{l}2,610 \\
1,880\end{array}$ \\
\hline 1955 & $\begin{array}{l}\text { June } 16,1955 \\
\text { June } 18, \quad 1955 \\
\end{array}$ & $\begin{array}{l}2.48 \\
2.25 \\
\end{array}$ & $\begin{array}{r}350 \\
242 \\
\end{array}$ & 1958 & $\begin{array}{l}\text { Apr. } 26,1958 \\
\text { May } \quad 3,1958 \\
\end{array}$ & $\begin{array}{l}1.65 \\
1.90 \\
\end{array}$ & $\begin{array}{l}310 \\
460 \\
\end{array}$ \\
\hline
\end{tabular}

a Maximum during period July 5 to Sept. 30, 1950; may have been exceeded during period of no record. 
Location.--Lat $32^{\circ} 48^{\prime} 26^{\prime \prime}$, long $97^{\circ} 14^{\prime} 54^{\prime \prime}$, on right bank at downstream side of bridge on State Highways 121 and $183,1.5$ miles upstream from Chicago, Rock Island and Pacific Railroad Co. bridge, 2.0 miles upstream from Little Fossil Creek, 3.5 miles upstream from mouth, and near east boundary of Ha.ltom City; Tarrant County.

Drainage area. $--52.8 \mathrm{sq} \mathrm{mi}$.

Gage.--Recording. Datum of gage $491.48 \mathrm{ft}$ above mean sea level, datum of 1929.

Stage-discharge relation.---Defined by current-meter measurements below 16,500 cfs and above by contracted-opening measurement of $27,000 \mathrm{cfs}$.

Bankfull stage. - - $13 \mathrm{ft}$.

Historical data.--Flood of Sept. 7, 1962, reached the highest stage since at least 1900.

Remarks.--Base for partial-duration series, $700 \mathrm{cfs}$. Rain gage at site.

Peak stages and discharges

\begin{tabular}{c|c|c|c||c|c|r|r}
\hline $\begin{array}{c}\text { Water } \\
\text { year }\end{array}$ & Date & $\begin{array}{c}\text { Gage } \\
\text { height } \\
\text { (feet) }\end{array}$ & $\begin{array}{c}\text { Discharge } \\
\text { (cfs) }\end{array}$ & $\begin{array}{c}\text { Water } \\
\text { year }\end{array}$ & Date & $\begin{array}{r}\text { Gage } \\
\text { height } \\
\text { (feet) }\end{array}$ & $\begin{array}{r}\text { Discharge } \\
\text { (cfs) }\end{array}$ \\
\hline 1960 & Oct. 1, 1959 & 20.71 & 12,600 & 1962 & July 27, 1962 & 18.68 & 5,410 \\
& Oct. 4, 1959 & 14.44 & 2,160 & & Aug. 2, 1962 & 19.13 & 6,180 \\
& Apr. 24, 1960 & 17.75 & 5,170 & & Sept. 5, 1962 & 9.38 & 750 \\
& May 6, 1960 & 12.94 & 1,560 & & Sept.7, 1962 & 24.90 & 27,000 \\
1961 & June 25, 1961 & 23.06 & 18,300 & 1963 & Nov. 27, 1962 & 11.53 & 1,220 \\
\hline
\end{tabular}

8-497. Walnut Creek near Mansfield, Tex.

(2)

Location.--Lat $32^{\circ} 34^{\prime} 50^{\prime \prime}$, long $97^{\circ} 06^{\prime} 05^{\prime \prime}$, on right bank at downistream side of bridge on county road, $2.6 \mathrm{miles}$ northeast of Mansfield; Tarrant County, 3 miles downstream from Texas and New Orleans Railroad Co. bridge, and 9 miles upstream from mouth.

Drainage area. $--62.8 \mathrm{sq} \mathrm{ml}$

Gage.--Recording. Datum of gage is $531.08 \mathrm{ft}$ above mean sea level, datum of 1929.

Stage-discharge relation.--Defined by current-meter measurements.

Bankfull stage. $--24 \mathrm{ft}$.

Historical data.--Maximum stage since at least 1900 occurred May 25, 1922, stage unknown.

Remarks.--Base for partial-duration series, $700 \mathrm{cfs}$. Rain gage at site.

\begin{tabular}{c|c|c|c||c|c|c|c}
\hline $\begin{array}{l}\text { Water } \\
\text { year }\end{array}$ & Date & $\begin{array}{c}\text { Gage } \\
\text { height } \\
\text { (feet) }\end{array}$ & $\begin{array}{c}\text { Discharge } \\
\text { (cfs) }\end{array}$ & $\begin{array}{c}\text { Water } \\
\text { year }\end{array}$ & Date & $\begin{array}{c}\text { Gage } \\
\text { height } \\
\text { (feet) }\end{array}$ & $\begin{array}{r}\text { Discharge } \\
\text { (cfs) }\end{array}$ \\
\hline 1961 & Jan. 8, 1961 & 17.20 & 1,350 & 1962 & Sept. 8, 1962 & 18.77 & 1,790 \\
& June 25, 1961 & 26.32 & 5,550 & 1963 & $\begin{array}{c}\text { Apr. 28, 1963 } \\
\text { Aug. 13, 1963 }\end{array}$ & $\begin{array}{r}19.69 \\
15.32\end{array}$ & 2,060 \\
& & & & 1960 \\
\hline
\end{tabular}

8-502. Elm Fork Trinity River subwatershed 6-0 near Muenster, Tex. (3)

Location.--Lat $33^{\circ} 37^{\prime} 13^{\prime \prime}$, long $97^{\circ} 24^{\prime} 15^{\prime \prime}$, near, center of earth-fill dam on unnamed tributary of EIm Fork Trinity River, 1.0 mile west of Farm Road 373 and 2.6 miles southwest of Muenster, Cooke County.

Drainage area.--0.77 sq $\mathrm{mi}$.

Gage.--Recording. Datum of gage is $941.75 \mathrm{ft}$ above mean sea level, datum of 1929 (U. S. Soil Conservation Service bench mark).

Remarks.--Peak discharge based on maximum inflow (average for 5 to 15-minute intervals), computed from outflow and change in reservoir contents, adjusted for rainfall on the reservoir surface during time of peak inflow. Only annual peaks are shown.

Peak stages and discharges

\begin{tabular}{c|c|c|c||c|c|c|c}
\hline $\begin{array}{c}\text { Water } \\
\text { year }\end{array}$ & Date & $\begin{array}{c}\text { Gage } \\
\text { height } \\
\text { (feet) }\end{array}$ & $\begin{array}{c}\text { Discharge } \\
\text { (cfs) }\end{array}$ & $\begin{array}{c}\text { Water } \\
\text { year }\end{array}$ & Date & $\begin{array}{c}\text { Gage } \\
\text { height } \\
\text { (feet) }\end{array}$ & $\begin{array}{c}\text { Discharge } \\
\text { (cfs) }\end{array}$ \\
\hline 1957 & June 1, 1957 & - & 449 & 1961 & Mar. 25, 1961 & - & \\
1958 & May 1, 1958 & - & 688 & 1962 & June 18, 1962 & - & 51 \\
1959 & Nov. 16, 1958 & - & 34 & 1963 & Nov. 26, 1962 & - & \\
1960 & Oct. 3, 1959 & - & 842 & & 221 \\
\hline
\end{tabular}


Location.--Lat $33^{\circ} 36^{\prime} 37^{\prime \prime}$, long $97^{\circ} 22^{\prime} 58^{\prime \prime}$, on left bank $40 \mathrm{ft}$ upstream from bridge on Farm Road 373 , $2.5 \mathrm{miles}$ south of Muenster, Cooke County, 2.5 miles downstream from Long Branch, and 6.5 miles upstream from Brushy Elm Creek.

Dra.inage area. $--46.0 \mathrm{sq} \mathrm{mi}$.

Gage.--Recording. Da.tum of gage is $889.33 \mathrm{ft}$ above mean sea. level, datum of 1929 .

Stage-discharge relation.--Defined by current-meter measurements below 1,300 cfs and above by slope-area measurement a.t $16.65 \mathrm{ft}(3,440 \mathrm{cfs})$.

Bankfull stage. $--16 \mathrm{ft}$.

Historical da.ta.--Maximum stage since at least 1900, about $23 \mathrm{ft}$, from information by loca.l resident.

Remarks.--Flow from $31.0 \mathrm{sq} \mathrm{mi}$ above this station partly controlled by $1 \mathrm{l}$ floodwater-retarding structures. Maximum outflow during period about $250 \mathrm{cfs}$. Only annual peaks are shown. Ruin gage at site.

\begin{tabular}{|c|c|c|c|c|c|c|c|}
\hline $\begin{array}{r}\text { Water } \\
\text { year }\end{array}$ & Date & $\begin{array}{c}\text { Gage } \\
\text { height } \\
\text { (feet) }\end{array}$ & $\begin{array}{c}\text { Discharge } \\
(\mathrm{cfs})\end{array}$ & $\begin{array}{r}\text { Water } \\
\text { year }\end{array}$ & Date & $\begin{array}{c}\text { Gage } \\
\text { height } \\
\text { (feet) }\end{array}$ & $\begin{array}{c}\text { Discharge } \\
\text { (cfs) }\end{array}$ \\
\hline $\begin{array}{l}1957 \\
1958 \\
1959 \\
1960\end{array}$ & $\begin{array}{lr}\text { Apr. } 26, & 1957 \\
\text { May 1, } 1958 \\
\text { June 23, } 1959 \\
\text { Oct. 3, } 1959\end{array}$ & $\begin{array}{r}15.20 \\
20.20 \\
3.95 \\
16.70\end{array}$ & $\begin{array}{r}3,480 \\
5,900 \\
33 \\
4,160\end{array}$ & $\begin{array}{l}1961 \\
1962 \\
1963\end{array}$ & $\begin{array}{l}\text { Mar. 25, } 1961 \\
\text { Sept. 7, } 1962 \\
\text { Nov. 26, } 1962\end{array}$ & $\begin{array}{r}7.40 \\
10.45 \\
10.26\end{array}$ & $\begin{array}{r}710 \\
1,310 \\
1,260\end{array}$ \\
\hline
\end{tabular}

\author{
8-527. Little Elm Creek near Aubrey, Tex. (18)
}

Location.--La.t $33^{\circ} 17^{\prime} 00^{\prime \prime}$, long $96^{\circ} 53^{\prime} 33^{\prime \prime}$, on left bank at downstream side of bridge on Farm Road 1385, 1 mile upstream from Mustang Creek, and 5.5 miles east of Aubrey, Denton County.

Drainage area. $--75.5 \mathrm{sq} \mathrm{mi}$.

Gage.--Recording. Da.tum of gage is $534.76 \mathrm{ft}$ above mean sea level (Sta.te Highway Department bench mark).

Stage-discharge relation.--Defined by current-meter measurements.

Bankfull stage. $--13 \mathrm{ft}$.

Historical da.ta.--Maximum stage known since about 1900, $18.2 \mathrm{ft}$ in May 1941, from information by local residents.

Remarks.--Base for partial-duration series, 1,000 cfs. Nine rain gages are in the basin above this station.

\begin{tabular}{|c|c|c|c|c|c|c|c|}
\hline $\begin{array}{r}\text { Wa.ter } \\
\text { year }\end{array}$ & - Date & $\begin{array}{c}\text { Gage } \\
\text { height } \\
\text { (feet) }\end{array}$ & $\begin{array}{c}\text { Discharge } \\
\text { (cfs) }\end{array}$ & $\begin{array}{r}\text { Water } \\
\text { yearr }\end{array}$ & Date & $\begin{array}{c}\text { Gage } \\
\text { height } \\
\text { (feet) }\end{array}$ & $\begin{array}{c}\text { Discharge } \\
\text { (cfs) }\end{array}$ \\
\hline 1957 & $\begin{array}{l}\text { Apr. 1, } 1957 \\
\text { Apr. 20, } 1957 \\
\text { Apr. 21, } 1957 \\
\text { Apr. 23, } 1957 \\
\text { Apr. 26, } 1957 \\
\text { May. 4, } 1957 \\
\text { May 13, } 1957 \\
\text { May 18, } 1957 \\
\text { May 22, } 1957 \\
\text { May 23, } 1957 \\
\text { May 25, } 1957\end{array}$ & $\begin{array}{l}14.70 \\
15.77 \\
14.28 \\
15.83 \\
17.34 \\
15.24 \\
16.87 \\
13.73 \\
14.22 \\
16.40 \\
16.57\end{array}$ & $\begin{array}{l}1,680 \\
3,300 \\
1,480 \\
3,520 \\
7,830 \\
2,420 \\
6,080 \\
1,200 \\
1,420 \\
4,830 \\
5,220\end{array}$ & $\begin{array}{l}1961 \\
1962\end{array}$ & $\begin{array}{l}\text { Oct. 4, } 1959 \\
\text { Nov. 4, } 1959 \\
\text { Dec. 16, } 1959 \\
\text { Jan. 8, } 1961 \\
\text { Apr. 24, } 1962 \\
\text { Apr. 28, } 1962 \\
\text { July 1, } 1962 \\
\text { Sept. 6, } 1962 \\
\text { Sept. 8, } 1962\end{array}$ & $\begin{array}{l}14.84 \\
15.10 \\
14.43 \\
14.60 \\
14.87 \\
14.31 \\
14.57 \\
16.48 \\
15.23\end{array}$ & $\begin{array}{l}1,650 \\
1,930 \\
1,310 \\
1,440 \\
1,680 \\
1,240 \\
1,420 \\
5,030 \\
2,120\end{array}$ \\
\hline 1958 & 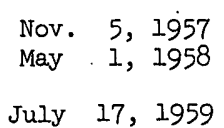 & $\begin{array}{l}15.55 \\
16.26 \\
11.29\end{array}$ & $\begin{array}{r}2,900 \\
4,460 \\
4.51\end{array}$ & 1963 & $\begin{array}{l}\text { Nov. } 27,1962 \\
\text { Apr. 29, } 1963 \\
\text { May } 31,1963\end{array}$ & $\begin{array}{l}14.45 \\
15.01 \\
15.17\end{array}$ & $\begin{array}{l}1,440 \\
1,820 \\
2,030\end{array}$ \\
\hline
\end{tabular}




\section{8-565. Turtle Creek at Dallas, Tex.(18)}

Location.--Lat $32^{\circ} 48^{\prime} 26^{\prime \prime}$, long $96^{\circ} 48^{\prime} 08^{\prime \prime}$, on left bank $68 \mathrm{ft}$ upstream from Hall Street Dam, $210 \mathrm{ft}$ upstream from Hall'street at Dallas, Dallas County, and 2.0 miles north of Dallas County courthouse.

Drainage area. $--7.98 \mathrm{sq} \mathrm{ml}$.

Gage.--Recording. Datum of gage is $428.13 \mathrm{ft}$ above mean sea level, unadjusted.

Stage-discharge relation.--Defined by current-meter measurements below 1,200 cf's and extended above on basis of weir formula, $Q=3.3 \mathrm{~L}(\mathrm{H})^{3 / 2}$.

Bankfull stage. $--4.5 \mathrm{ft}$.

Historical data.--Flood of oct. 1, 1959, reached the highest stage since at least 1903 .

Remarks.--Base for partial-duration series, $880 \mathrm{cfs}$. The creek basin is in a highly-developed urban area.

Peak stages and discharges

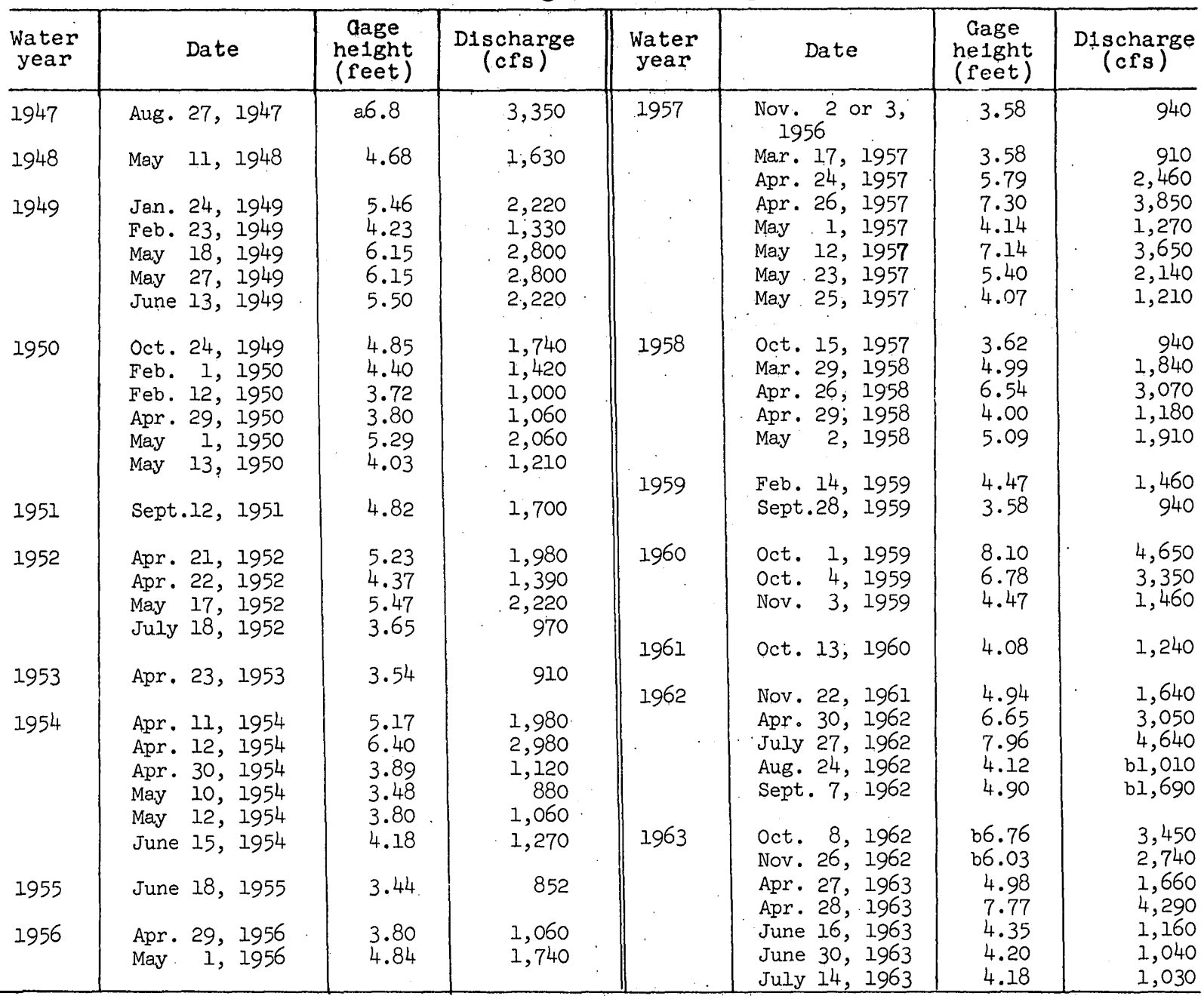

a Annual peak only.

b Stage-discharge relation indefinite. 
Location.--Lat $32^{\circ} 58^{\prime} 13^{\prime \prime}$, long $96^{\circ} 48^{\prime} 19^{\prime \prime}, 20 \mathrm{ft}$ left of left abutment of bridge on Keller Springs Rd., 0.5 mile upstream from St. Louis and Southwestern Railroad Co. bridge, 0.9 mile upstream from Spanky Branch, and 13.0 miles north of City Hall of Dallas, Dallas County.

Drainage area.--29.4 sq mi.

Gage---Recording. Datum of gage is mean sea level, datum of 1929, supplementary adjustment of 1953.

Stage-discharge relation. --Defined by current-meter measurements below 5,400 cfs and above by contracted-opening measurement of $38,000 \mathrm{cfs}$.

Bankfull stage. --562 ft.

Historical data.--Historical flood data begins in 1886 . The highest stage of $569.6 \mathrm{ft}$, occurred Apr. 19, 1942, from information by local resident (flood Sept. 21, 1964 reached a stage of $574.51 \mathrm{ft})$.

Remarks.--Base for partial-duration series, $1,500 \mathrm{cfs}$. Three recording rain gages are in the basin upstream from this station.

\begin{tabular}{c|c|c|c||c|c|c|c}
\hline $\begin{array}{c}\text { Water } \\
\text { year }\end{array}$ & Date & $\begin{array}{c}\text { Gage } \\
\text { height } \\
\text { (feet) }\end{array}$ & $\begin{array}{c}\text { Discharge } \\
\text { (cfs) }\end{array}$ & $\begin{array}{c}\text { Water } \\
\text { year }\end{array}$ & Date & $\begin{array}{c}\text { Gage } \\
\text { height } \\
\text { (feet) }\end{array}$ & $\begin{array}{c}\text { Discharge } \\
\text { (cfs) }\end{array}$ \\
\hline 1962 & June 27, 1962 & 558.43 & 3,550 & 1963 & 0ct. 8, 1962 & 555.46 & 2,360 \\
& June 29, 1962 & 560.73 & 4,560 & & Oct. 28, 1962 & 556.11. & 2,620 \\
\hline
\end{tabular}

8-571.2 Spanky Branch at McCallum Lane, Dallas, Tex. (18)

Location.--Lat $32^{\circ} 57^{\prime} 58^{\prime \prime}$, long $96^{\circ} 48^{\prime} 11^{\prime \prime}$, at downstream side of bridge on McCallum Lane, 0.2 mile upstream from State Highway 289, 0.5 mile upstream from mouth, and 12.7 miles north of City Hall of Dallas, Dallas County.

Drainage area.--6.77 sq mi.

Gage.--Crest-stage gage. Datum of gage is mean sea level, datum of 1929, supplementary adjustment of 1953.

Stage-discharge relation.--Defined by current-meter measurements below $365 \mathrm{cfs}$ and above by indirect measurements.

Bankfull stage. $--565 \mathrm{ft}$.

Historical data.--Maximum stage since at least 1917 occurred Apr. 19, 1942 (flood of Sept. 21, 1964 exceeded Apr. 19, 1942. Stage 572.0, discharge 7,820 cfs).

Remarks.--Base for partial-duration series, $600 \mathrm{cfs}$. Two recording rain gages are in the basin upstream from the station.

\begin{tabular}{c|c|c|c||c|c|c|c}
\hline $\begin{array}{c}\text { Water } \\
\text { year }\end{array}$ & Date & $\begin{array}{c}\text { Gage } \\
\text { height } \\
\text { (feet) }\end{array}$ & $\begin{array}{c}\text { Discharge } \\
\text { (cfs) }\end{array}$ & $\begin{array}{c}\text { Water } \\
\text { year }\end{array}$ & Date & $\begin{array}{c}\text { Gage } \\
\text { height } \\
\text { (feet) }\end{array}$ & $\begin{array}{c}\text { Discharge } \\
\text { (cfs) }\end{array}$ \\
\hline 1962 & July 27, 1962 & 567.03 & 4,020 & 1963 & Oct. 8, 1962 & 564.61 & 3,000 \\
& Sept.7, 1962 & 559.23 & 1,400 & & Oct. 28, 1962 & 558.98 & 1,320 \\
\hline
\end{tabular}

8-571.4 Cottonwood Creek at Forest Lane, Dallas; Tex. (18)

Location.--Lat $32^{\circ} 54^{\prime} 33^{\prime \prime}$, long $96^{\circ} 45^{\prime} 54^{\prime \prime}$, at downstream side of bridge on Forest Lane, 0.2 mile east of U. S. Highway 75 (Central Expressway), 0.2 mile upstream from Floyd Branch, 0.7 mile upstream from mouth, and 8.9 miles northeast of City Hall of Dalla.s, Dallas County.

Drainage area.---8.50 sq mi.

Gage.--Crest-stage gage. Flood hydrograph recorder installed in 1963. Datum of gage is mean sea level, datum of 1929, supplementary adjustment of 1953.

Stage-discharge relation.--Defined by current-meter measurements below 328 cfs and above by indirect measurements.

Bankfull stage. --507 ft.

Historical data.--Maximum stage since at least 1892, $512.5 \mathrm{ft}$ on June 13, 1949, at upstream side of bridge. Information from local residents and records at the county engineer's office.

Remarks.--Base for partial-record series, $370 \mathrm{cfs}$. There are 2 recording-rain gages in the basin upstream from the station. 
TRINITY RIVER BASIN

Peak stages and discharges of Cottonwood Creek at Forest Lane, Dallas, Tex.

\begin{tabular}{c|c|c|r||c|c|c|c}
\hline $\begin{array}{c}\text { Water } \\
\text { year }\end{array}$ & Date & $\begin{array}{c}\text { Gage } \\
\text { height } \\
\text { (feet) }\end{array}$ & $\begin{array}{c}\text { Discharge } \\
\text { (cfs) }\end{array}$ & $\begin{array}{c}\text { Water } \\
\text { year }\end{array}$ & Da.te & $\begin{array}{c}\text { Gage } \\
\text { height } \\
\text { (feet) }\end{array}$ & $\begin{array}{c}\text { Discharge } \\
\text { (cfs) }\end{array}$ \\
\hline 1962 & Nov. 22, 1961 & 503.55 & 870 & 1963 & Oct. 8, 1962 & 511.74 & 17,400 \\
& June 29, 1962 & 502.96 & 780 & & Oct. 28, 1962 & 501.90 & \\
& July 27, 1962 & 509.90 & 5,090 & & Apr. 28, 1963 & 500.56 & \\
& Sept.7, 1962 & 502.37 & 585 & & 485 \\
\hline
\end{tabular}

8-571.6 Floyd Branch at. Forest Lane, Dallas, Tex. (18)

Location.--Lat $32^{\circ} 54^{\prime} 33^{\prime \prime}$, long 96 $45^{\prime} 34^{\prime \prime}$, at downstream side of bridge on Forest Lane, $0.3 \mathrm{mile}$ upstream from mouth, 0.5 mile east of U.. S. Highway 75 (Central Expressway), and 9.0 miles northeast of City Hall of Dallas, Dallas County.

Drainage area.-- $-4.17 \mathrm{sq} \mathrm{mi}$.

Gage.--Crest-stage gage. Datum of gage is mean sea level, datum of 1929, supplementary adjustment of 1953.

Stage-discharge relation.--Defined by current-meter measurements below 135 cfs and above by indirect measurements.

Bankfull stage. --508 ft.

Historical data.--Maximum stage since at least 1909, $513.7 \mathrm{ft}$ on June 13, 1949, from information by local residents.

Remarks.--Base for partial-record series, 1,300 cfs.

\begin{tabular}{c|c|c|c|c|c|c|c}
\hline $\begin{array}{c}\text { Water } \\
\text { year }\end{array}$ & Date & $\begin{array}{c}\text { Gage } \\
\text { height } \\
\text { (feet) }\end{array}$ & $\begin{array}{c}\text { Discharge } \\
\text { (cfs) }\end{array}$ & $\begin{array}{c}\text { Water } \\
\text { year }\end{array}$ & Date & $\begin{array}{c}\text { Gage } \\
\text { height } \\
\text { (feet) }\end{array}$ & $\begin{array}{c}\text { Discharge } \\
\text { (cfs) }\end{array}$ \\
\hline 1962 & $\begin{array}{c}\text { June 29, 1962 } \\
\text { July 27, 1962 }\end{array}$ & $\begin{array}{c}505.66 \\
509.62\end{array}$ & $\begin{array}{c}2,000 \\
3,200\end{array}$ & 1963 & Oct. 8, 1962 & 512.63 & 4,850 \\
\hline
\end{tabular}

8-572. White Rock Creek at Greenville Ave., Dallas, Tex. (18)

Location.--Lat $32^{\circ} 53^{\prime} 2 l^{\prime \prime}$, long $96^{\circ} 45^{\prime} 23^{\prime \prime}$, on left bank, $20 \mathrm{ft}$ downstream from bridge on Greenville Ave., 1.1 miles downstream from Texas and New Orleans Railroad, 1.2 miles downstream from Cottonwood Creek, 2.9 miles upstream from White Rock Lake, and 7.7 miles northeast of City Hall of Dallas, Dallas County.

Drainage area.--66.4 sq mi.

Gage---Recording. Datum of gage is mean sea level, datum of 1929, supplementary adjustment of 1953.

Stage-discharge relation.--Defined by current-meter measurements.

Bankfull stage. $-483 \mathrm{ft}$.

Historical data.--Historical flood data begins in 1886. The highest stage of 490.1 ft occurred Apr. 19, 1942 (flood of Sept. 21, 1964 reached a stage of $490.4 \mathrm{ft}$ ).

Remarks.--Base for partial-duration series, 2,900 cfs. Twelve recording-rain gages are in the basin upstream from the station.

\begin{tabular}{c|c|c|c||c|c|c|c}
\hline $\begin{array}{c}\text { Water } \\
\text { year }\end{array}$ & Da.te & $\begin{array}{c}\text { Gage } \\
\text { height } \\
\text { (feet) }\end{array}$ & $\begin{array}{c}\text { Discharge } \\
\text { (cfs) }\end{array}$ & $\begin{array}{c}\text { Water } \\
\text { year }\end{array}$ & Date & $\begin{array}{c}\text { Gage } \\
\text { height } \\
\text { (feet) }\end{array}$ & $\begin{array}{c}\text { Discharge } \\
\text { (cfs) }\end{array}$ \\
\hline 1962 & Nov. 22, 1961 & 483.42 & 2,960 & 1963 & Oct. 8, 1962 & 489.23 & 24,500 \\
& June 29, 1962 & 487.43 & 7,980 & & Oct. 28, 1962 & 487.40 & 6,900 \\
& July 27, 1962 & 488.80 & 2,000 & & Apr. 28, 1963 & 485.64 & 3,980 \\
& Sept.8, 1962 & 486.49 & 5,000 & & & & \\
\hline
\end{tabular}


Location.--Lat $32^{\circ} 48^{\prime} 18^{\prime \prime}$, long $96^{\circ} 43^{\prime} 04^{\prime \prime}$, on downstream side of bridge on Highland Road, $0.4 \mathrm{mile}$ upstream from White Rock Creek, in Dallas, Dallas County.

Drainage area. $--6.92 \mathrm{sq} \mathrm{mi}$.

Gage.--Crest-stage gage. Flood hydrograph recorder installed in 1963. Da.tum of gage is mean sea level, datum of 1929, supplementary adjustment of 1961.

Stage-discharge relation.--Not defined.

Bankfull stage.--429 ft.

Historical data.--Historical data are limited. Channel rectification has altered flow conditions. Maximum stage since channel work, $433.4 \mathrm{ft}$, Apr. 26, 1957, from Dallas Public Works Department.

Remarks.--Base for partial-duration series, $427.31 \mathrm{ft}$.

Peak stages and discharges

\begin{tabular}{c|c|c|c||c|c|c|c}
\hline $\begin{array}{r}\text { Water } \\
\text { year }\end{array}$ & Date & $\begin{array}{c}\text { Gage } \\
\text { height } \\
\text { (feet) }\end{array}$ & $\begin{array}{c}\text { Discharge } \\
(\mathrm{cfs})\end{array}$ & $\begin{array}{c}\text { Water } \\
\text { year }\end{array}$ & $\begin{array}{c}\text { Gage } \\
\text { height } \\
\text { (feet) }\end{array}$ & $\begin{array}{c}\text { Discharge } \\
\text { (cfs) }\end{array}$ \\
\hline 1963 & Apr. 28, 1963 & 430.99 & - & & & & \\
\hline
\end{tabular}

8-573.4 Forney Creek at Lawnview Ave., Dallas, Tex. (18).

Location.--Lat $32^{\circ} 46^{\prime} 45^{\prime \prime}$, long $96^{\circ} 40^{\prime} 45^{\prime \prime}$, at downstream side of culvert on Lawnview Ave., 0.8 mile upstream from White Rock Creek, in Dallas, Dallas County.

Drainage area.--1.84 sq $\mathrm{mi}$.

Gage.--Crest-stage gage. Datum of gage is mean sea level, datum of 1929, supplementary adjustment of 1953.

Stage-discharge relation.--Not defined.

Bankfull stage.--432 ft.

Historical da.ta.--Flood history is scarce. From data furnished by employees of Dallas Department of Public Works, it is indicated that stages in excess of $435 \mathrm{ft}$ occurred Apr. 19, 1942 and June 13, 1949.

Remarks.--Base for partial-duration series, $429.01 \mathrm{ft}$.

Peak stages and discharges

\begin{tabular}{c|c|c|c||c|c|c|c}
\hline $\begin{array}{c}\text { Water } \\
\text { year }\end{array}$ & Date & $\begin{array}{c}\text { Gage } \\
\text { height } \\
\text { (feet) }\end{array}$ & $\begin{array}{c}\text { Discharge } \\
\text { (cfs) }\end{array}$ & $\begin{array}{c}\text { Water } \\
\text { year }\end{array}$ & $\begin{array}{c}\text { Gage } \\
\text { height } \\
\text { (feet) }\end{array}$ & $\begin{array}{c}\text { Discharge } \\
\text { (cfs) }\end{array}$ \\
\hline 1963 & Apr. 28,1963 & 431.36 & - & & & \\
\hline
\end{tabular}


Location.--Lat $33^{\circ} 18^{\prime} 10^{\prime \prime}$, long $96^{\circ} 41^{\prime} 30^{\prime \prime}$, near center of dam on unnamed tributary of Honey Creek, 1.5 miles west of Farm Road 543 and 8.4 miles northwest of McKinney, Collin County.

Dra1nage area. $--2.14 \mathrm{sq} \mathrm{ml}$.

Gage.--Recording. Datum of gage is 629.0.0 $\mathrm{ft}$ above.mean sea level, datum of 1929.

Remarks.--Peak discharge based on maximum inflow (average for 15-minute Interval), computed from outflow and change in reservolr contents, adjusted for rainfall on the reservolr surface during time of peak inflow. Base for partial-duration series, $200 \mathrm{cfs}$. Only annual peaks shown beginning in 1960.

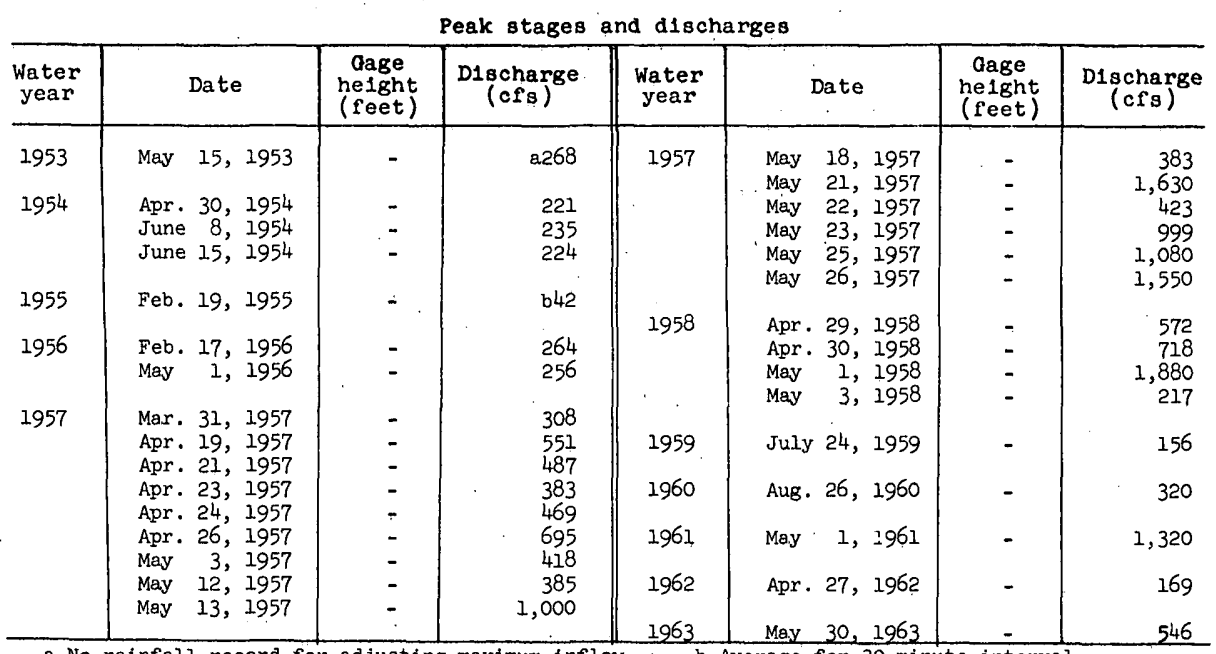

a No rainfall record for adjusting maximum inflow. b Average for 30-minute interval.

8-580. Honey Creek subwatershed No. 12 near McKinney, Tex.(18)

Location.--Lat $33^{\circ} 18^{\prime} 20^{\prime \prime}$, long $96^{\circ} 40^{\prime} 15^{\prime \prime}$, near center of dam on unnamed tributary of Honey Creek, 0.5 mile west of Farm Road 543 and 7.8 miles northwest of McKinney, Collin County.

Drainage area. $--1.26 \mathrm{sq} \mathrm{ml}$.

Gage.--Recording. Datum of gage is $623.00 \mathrm{ft}$ above mean sea level, datum of T929.

Remarks.--Peak discharge based on maximum inflow (average for 15-minute

interval), computed from outflow and change in reservoir contents, adjusted for rainfall on the reservoir surface during time of peak inflow. Base for

partial-duration series, $150 \mathrm{cfs}$. Only annual peaks shown beginning in 1960.

\begin{tabular}{|c|c|c|c|c|c|c|c|}
\hline $\begin{array}{l}\text { Water } \\
\text { year }\end{array}$ & Date & $\begin{array}{c}\text { Gage } \\
\text { nelght } \\
\text { (feet) }\end{array}$ & $\begin{array}{c}\text { Discharge } \\
(\text { cfs })\end{array}$ & $\begin{array}{l}\text { Water } \\
\text { year }\end{array}$ & Date & $\begin{array}{c}\text { Gage } \\
\text { he1ght } \\
(\text { feet }) \\
\end{array}$ & $\begin{array}{c}\text { D1scharge } \\
\text { (cfs) }\end{array}$ \\
\hline 1953 & $\begin{array}{l}\text { Apr. } 28,1953 \\
\text { May } 15,1953 \\
\\
\text { June } 8,1954 \\
\text { June } 15,1954\end{array}$ & $\begin{array}{l}- \\
-\end{array}$ & $\begin{array}{r}0423 \\
0.194 \\
196 \\
212\end{array}$ & 1957 & $\begin{array}{lll}\text { May } & 21,1957 \\
\text { May } & 22,1957 \\
\text { May } & 23,1957 \\
\text { May } & 25,1957 \\
\text { May } & 26,1957\end{array}$ & $\begin{array}{l}- \\
- \\
-\end{array}$ & $\begin{array}{r}1,490 \\
340 \\
891 \\
1,110 \\
1,420\end{array}$ \\
\hline $\begin{array}{l}1955 \\
1956 \\
1957\end{array}$ & $\begin{array}{l}\text { Oct. 23, } 1954 \\
\text { Feb. 17, } 1956\end{array}$ & - & $\begin{array}{l}\text { b123 } \\
\text { b295 } \\
824\end{array}$ & 1958 & $\begin{array}{l}\text { Apr. } 29,1958 \\
\text { Apr. } 30,1958 \\
\text { May } 1,1958 \\
\text { May } \\
\text { 3, } 1958\end{array}$ & $\begin{array}{l}- \\
-\end{array}$ & $\begin{array}{r}1,170 \\
744 \\
1,410 \\
190\end{array}$ \\
\hline 1957 & $\begin{array}{l}\text { Apr. 19, } 1957 \\
\text { Apr. } 21,1957 \\
\text { Apr. } 23,1957 \\
\text { Apr. } 24,1957 \\
\text { Apr. } 25,1957 \\
\text { Apr. } 26,1957 \\
\text { May } 3,1957 \\
\text { May } 13,1957 \\
\text { May 16, } 1957 \\
\text { May 18,1957 }\end{array}$ & $\begin{array}{l}- \\
- \\
- \\
- \\
- \\
-\end{array}$ & $\begin{array}{l}824 \\
247 \\
544 \\
581 \\
296 \\
674 \\
406 \\
523 \\
176 \\
422 \\
\end{array}$ & $\begin{array}{l}1959 \\
1960 \\
1961 \\
1962 \\
1963 \\
\end{array}$ & $\begin{array}{l}\text { July } 24,1959 \\
\text { June } 8,1960 \\
\text { May } 1,1961 \\
\text { Apr. 24, } 1962 \\
\text { May } 30,1963\end{array}$ & $\begin{array}{l}- \\
- \\
- \\
-\end{array}$ & $\begin{array}{l}(c) \\
286 \\
589 \\
158 \\
663 \\
\end{array}$ \\
\hline
\end{tabular}




\section{TRINITY RIVER BASIN}

\section{8-585. Honey. Creek near McKInney, Tex.(18)}

Location.--Lat $33^{\circ} 171$, long $96^{\circ} 39^{\prime}$, on right bank at downstream side of bridge, 4.5 mlles downstream from Haw Branch, 5.6 miles upstream from mouth, and 6.0 miles northwest of McKinney, Coliin County.

Dralnage area. $--39.0 \mathrm{sq} \mathrm{m} 1$.

Gage.--Recording. Datum of gage is $563.68 \mathrm{ft}$ above mean sea level, datum of I.929.

Stage-discharge relation.--Defined by current-meter measurements below $4,620 \mathrm{cfs}$.

Bankfull stage. $--14 \mathrm{ft}$.

H1storical data.--Flood in 1950 (probably. June) reached highest stage since at least 1930, from information by local resident.

Remarks.--Between 1951 and July 1957, 12 floodwater-retarding structures were built. These structures have a total floodwater-detention capacity of 8,320 acre-ft below the flood splliway crests and partly control the flow from $20.9 \mathrm{sq} \mathrm{mi}$ above the station. Only annual peaks are shown.

Peak stages and discharges

\begin{tabular}{|c|c|c|c|c|c|c|c|}
\hline $\begin{array}{l}\text { Water } \\
\text { year }\end{array}$ & Date & $\begin{array}{c}\text { Qage } \\
\text { nelght } \\
\text { (feet) }\end{array}$ & $\begin{array}{c}\text { Discharge } \\
(\mathrm{cfs})\end{array}$ & $\begin{array}{l}\text { Water } \\
\text { year }\end{array}$ & Date & $\begin{array}{c}\text { Gage } \\
\text { helght } \\
\text { (feet) }\end{array}$ & $\begin{array}{c}\text { D1scharge } \\
(\mathrm{cfs})\end{array}$ \\
\hline $\begin{array}{l}1950 \\
1951 \\
1952 \\
1953 \\
1954 \\
1955\end{array}$ & $\begin{array}{l}\text { June } 1950 \\
\text { Aug. } 23,1951 \\
\text { Apr. 22, } 1952 \\
\text { May 15, } 1953 \\
\text { May 12, } 1954 \\
\text { Feb. 19, } 1955\end{array}$ & $\begin{array}{l}23.0 \\
a 1.55 \\
16.65 \\
16.65 \\
16.66 \\
15.74\end{array}$ & $\begin{array}{c}- \\
2,500 \\
2,8 \\
2,540 \\
2,510 \\
2,050\end{array}$ & $\begin{array}{l}1956 \\
1957 \\
1958 \\
1959 \\
1960 \\
1961 \\
1962 \\
1963 \\
\end{array}$ & $\begin{array}{lrl}\text { May } & 1, & 1956 \\
\text { May } & 26, & 1957 \\
\text { May } & 2, & 1958 \\
\text { June } & 23, & 1959 \\
\text { Nov. } & 4, & 1959 \\
& \\
\text { May } & 1, & 1961 \\
\text { Sept. } 6, & 1962 \\
\text { May } 30, & 1963 \\
\end{array}$ & $\begin{array}{r}14.27 \\
20.29 \\
18.70 \\
7.76 \\
11.81 \\
13.10 \\
13.52 \\
17.08 \\
\end{array}$ & $\begin{array}{r}1,410 \\
7,920 \\
4,580 \\
384 \\
982 \\
1,280 \\
1,390 \\
2,920 \\
\end{array}$ \\
\hline
\end{tabular}

a Maximum for period July to September 1951; probably exceeded during period of no record. Note.--Peak discharge for May 26, 1957 includes undetermined amount of flow over emergency spiliways of floodwater-retarding structures. Other peak discharges given since 1957 include up to about $150 \mathrm{cfs}$ of combined service spillway discharge. 
8-616.45. Unnamed tributary (watershed WI) of Duck Creek near Garland, Tex. (18)

Location.--Lat $32^{\circ} 50^{\prime}$, long $96^{\circ} 37^{\prime}, 5.6$ miles south of Garland, Dallas County. Drainage area. $--0.0391 \mathrm{sq} \mathrm{m}$.

Gage.--Recording.

Remarks.--Records furnished by U.S. Department of Agriculture, Agricultural Research Service. Only annual (calendar year) peaks are shown.

\begin{tabular}{|c|c|c|c|c|c|c|c|}
\hline $\begin{array}{l}\text { Calen- } \\
\text { dar } \\
\text { year }\end{array}$ & Date & $\begin{array}{c}\text { Gage } \\
\text { nelght } \\
\text { (feet) }\end{array}$ & $\begin{array}{c}\text { Discharge } \\
\text { (cfs) }\end{array}$ & $\begin{array}{l}\text { Calen- } \\
\text { dar } \\
\text { year }\end{array}$ & Date & $\begin{array}{c}\text { Gage } \\
\text { helght } \\
\text { (feet) }\end{array}$ & $\begin{array}{c}\text { Discharge } \\
\text { (cfs) }\end{array}$ \\
\hline $\begin{array}{l}1939 \\
1940\end{array}$ & $\begin{array}{l}\text { June } 19,1939 \\
\text { Apr. }\end{array}$ & $\overline{-}$ & $\begin{array}{l}1.0 \\
9.6\end{array}$ & $\begin{array}{l}1943 \\
1944 \\
1945\end{array}$ & $\begin{array}{lr}\text { June } & 6,1943 \\
\text { May } 1, & 1944 \\
\text { Mar. } 30, & 1945\end{array}$ & $\overline{-}$ & $\begin{array}{l}42 \\
5.0 \\
32\end{array}$ \\
\hline $\begin{array}{l}1941 \\
1942 \\
\end{array}$ & $\begin{array}{l}\text { June } 13,1941 \\
\text { Apr. } 20,1942 .\end{array}$ & - & $\begin{array}{l}6.6 \\
29 \\
\end{array}$ & 1946 & May 29,1946 & - & \\
\hline
\end{tabular}

8-616.5. Unnamed tributary (watershed WIII) of Duck Creek near Garland, Tex. (18)

Location.--Lat $32^{\circ} 50^{\prime}$, long $96^{\circ} 37^{\prime}, 5.1$ miles south of Garland, Dallas County. Drainage area. $--0.0162 \mathrm{sq} \mathrm{ml}$.

Gage.--Recording.

Remarks.--Records furnished by U.S. Department of Agriculture, Agricultural Research Service. Only annual (calendar year) peaks are shown.

Peak stages and discharges

\begin{tabular}{l|c|c|c||c|c|c|c}
\hline $\begin{array}{c}\text { Calen- } \\
\text { dar } \\
\text { year }\end{array}$ & Date & $\begin{array}{c}\text { Gage } \\
\text { helght } \\
\text { (feet) }\end{array}$ & $\begin{array}{c}\text { D1scharge } \\
\text { (cfs) }\end{array}$ & $\begin{array}{c}\text { Calen- } \\
\text { dar } \\
\text { year }\end{array}$ & Date & $\begin{array}{c}\text { Gage } \\
\text { he1ght } \\
\text { (feet) }\end{array}$ & $\begin{array}{c}\text { D1scharge } \\
\text { (cfs) }\end{array}$ \\
\hline 1939 & Mar. 29, 1939 & - & 0.3 & 1943 & June 6, 1943 & - & 36 \\
1940 & Dec. 31, 1940 & - & 20 & 1944 & May 1, 1944 & - & 7.2 \\
1941 & Apr. 22, 1941 & - & 15 & 1945 & Mar. 29, 1945 & - & 25 \\
1942 & Apr. 20, 1942 & - & 41 & 1946 & May 29, 1946 & - & 45 \\
\hline
\end{tabular}

8-616.55. Unnamed tributary (watershed WIV) of Duck Creek near Garland, Tex. (18)

Location.--Lat $32^{\circ} 50^{\prime}$, long $96^{\circ} 37^{\prime}, 5.8$ miles south of Garland, Dallas County. Drainage area. $-0.0253 \mathrm{sq} \mathrm{ml}$.

Gage.--Recording.

Remarks.--Records furnished by U.S. Department of Agriculture, Agricultural

Research Service. Only annual (calendar year) peaks are shown.

\begin{tabular}{|c|c|c|c|c|c|c|c|c|}
\hline $\begin{array}{l}\text { Calen- } \\
\text { dar } \\
\text { year } \\
\end{array}$ & Date & $\begin{array}{c}\text { Gage } \\
\text { ne1ght } \\
\text { (feet) }\end{array}$ & $\begin{array}{c}\text { Discharge } \\
\text { (cfs) }\end{array}$ & $\begin{array}{l}\text { Calen- } \\
\text { dar } \\
\text { year }\end{array}$ & & Date & $\begin{array}{c}\text { Gage } \\
\text { nelght } \\
\text { (feet) }\end{array}$ & $\begin{array}{c}\text { Discharge } \\
\text { (cfs) }\end{array}$ \\
\hline 1939 & Apr. 16, 1939 & - & 19 & $\begin{array}{l}1944 \\
1945\end{array}$ & $\begin{array}{l}\text { May } \\
\text { June }\end{array}$ & $\begin{array}{ll}30, & 1944 \\
12, & 1945\end{array}$ & - & $\begin{array}{l}37 \\
49\end{array}$ \\
\hline $\begin{array}{l}1940 \\
1941 \\
1942\end{array}$ & $\begin{array}{l}\text { May } 17,1940 \\
\text { Oct. } 1,1941 \\
\text { May } 19,1942\end{array}$ & $\overline{-}$ & $\begin{array}{l}44 \\
21 \\
57\end{array}$ & 1946 & May & 29,1946 & - & 121 \\
\hline
\end{tabular}


Location.--Lat $32^{\circ} 50^{\prime} 00^{\prime \prime}$, long $96^{\circ} 35^{\prime} 45^{\prime \prime}$, on right bank at downstream side of bridge on Belt Line Road, 6.0 miles southeast of Garland, Dallas County.

Drainage area. $--31.6 \mathrm{sq} \mathrm{ml}$.

Gage.--Recording. Datum of gage is $430.02 \mathrm{ft}$ above mean sea level, datum of 1929, adjustment of 1954. Prior to Oct. 1, 1962, at datum $4.00 \mathrm{ft}$ higher.

Stage-discharge relation.---Defined by current-meter measurements.

Bankfull stage. --12 ft.

Historical data.--Maximum stage since about 1895, $21.5 \mathrm{ft}$, present datum, June 13, 1949, from information by local residents.

Remarks.--Base for partial-duration series, 1,000 cfs. Rain gage a.t site.

\begin{tabular}{|c|c|c|c|c|c|c|c|}
\hline $\begin{array}{r}\text { Water } \\
\text { year }\end{array}$ & Date & $\begin{array}{c}\text { Gage } \\
\text { height } \\
\text { (feet) }\end{array}$ & $\begin{array}{l}\text { Discharge } \\
\text { (cfs) }\end{array}$ & $\begin{array}{l}\text { Water } \\
\text { year }\end{array}$ & Date & $\begin{array}{l}\text { Gage } \\
\text { height } \\
\text { (feet) }\end{array}$ & $\begin{array}{l}\text { Discharge } \\
\text { (cfs) }\end{array}$ \\
\hline 1958 & $\begin{array}{l}\text { Mar. 29, } 1958 \\
\text { Apr. 26, } 1958 \\
\text { Apr. 29, } 1958 \\
\text { Apr. } 30,1958\end{array}$ & $\begin{array}{l}12.95 \\
14.12 \\
11.77 \\
11.63\end{array}$ & $\begin{array}{l}4,060 \\
7,400 \\
2,140 \\
2,030\end{array}$ & 1961 & $\begin{array}{l}\text { Jan. } 8,1961 \\
\text { Mar. 17, } 1961 \\
\text { June } 25,1961\end{array}$ & $\begin{array}{l}11.08 \\
10.38 \\
11.79\end{array}$ & $\begin{array}{l}1,580 \\
1,160 \\
2,080\end{array}$ \\
\hline & May 2, 1958 & 12.40 & 3,040 & 1962 & $\begin{array}{l}\text { Nov. } 22,1961 \\
\text { Dec. } 9,1961\end{array}$ & $\begin{array}{l}11.52 \\
10.64\end{array}$ & $\begin{array}{l}1,760 \\
1,090\end{array}$ \\
\hline 1959 & Feb. 14, 1959 & 11.93 & 2,380 & & $\begin{array}{l}\text { Apr. 23, } 1962 \\
\text { Apr. 27, } 1962\end{array}$ & $\begin{array}{l}10.54 \\
11.44\end{array}$ & $\begin{array}{l}1,030 \\
1,670\end{array}$ \\
\hline 1960 & $\begin{array}{l}\text { Oct. } 1,1959 \\
\text { Oct. } 4,1959 \\
\text { Nov. } 4,1959 \\
\text { Dec. } 15,1959 \\
\text { Jan. } 5,1960\end{array}$ & $\begin{array}{l}13.00 \\
13.30 \\
11.62 \\
10.84 \\
10.76\end{array}$ & $\begin{array}{l}4,160 \\
4,820 \\
1,980 \\
1,420 \\
1,360\end{array}$ & & $\begin{array}{l}\text { Apr. 30, } 1962 \\
\text { June 29, } 1962 \\
\text { July 27, } 1962 \\
\text { Sept. 8, } 1962\end{array}$ & $\begin{array}{l}11.97 \\
13.56 \\
16.80 \\
12.87\end{array}$ & $\begin{array}{r}2,300 \\
5,620 \\
16,000 \\
3,730\end{array}$ \\
\hline 1961 & $\begin{array}{l}\text { Aug. } 21,1960 \\
\text { Dec. } 10,1960\end{array}$ & $\begin{array}{l}10.70 \\
10.24\end{array}$ & $\begin{array}{l}1,330 \\
1,070\end{array}$ & 1963 & $\begin{array}{l}\text { Oct. } 8,1962 \\
\text { Oct. } 28,1962 \\
\text { Nov. 26, } 1962 \\
\text { Apr. 28, } 1963\end{array}$ & $\begin{array}{l}18.65 \\
15.03 \\
17.18 \\
18.20\end{array}$ & $\begin{array}{l}8,600 \\
1,350 \\
4,480 \\
7,400\end{array}$ \\
\hline
\end{tabular}

8-632. Pin Oak Creek near Hubbard, Tex. (9)

Location.--Lat $31^{\circ} 48^{\prime} 05^{\prime \prime}$, long $96^{\circ} 43^{\prime} 10^{\prime \prime}$, on right bank $85^{\mathrm{ft}}$ downstream from bridge on State Highway $171,5.8$ miles southeast of Hubbard, Hill County.

Drainage area. --17.6 sq mi.

Gage.--Recording. Datum of gage is $463.08 \mathrm{ft}$ above mean sea level, datum of 1929, supplementary adjustment of 1942 .

Stage-discharge relation.--Defined by current-meter measurements below 2,900 cfs.

Bankfull stage. - - $15 \mathrm{ft}$.

Historical data.--Maximum stage since at least 1900, about $17 \mathrm{ft}$ in August 1919, from information by local resident.

Remarks.--Base for partial-duration series, $800 \mathrm{cfs}$. Flood-retarding structures partially controlling $7.29 \mathrm{sq} \mathrm{mi}$ above this station were built during 1963. Six rain gages are operated in the basin above this station.

Peak stages and discharges

\begin{tabular}{|c|c|c|c|c|c|c|c|}
\hline $\begin{array}{r}\text { Water } \\
\text { year }\end{array}$ & Date & $\begin{array}{c}\text { Gage } \\
\text { height } \\
\text { (feet) }\end{array}$ & $\begin{array}{l}\text { Discharge } \\
\text { (cfs) }\end{array}$ & $\begin{array}{l}\text { Water } \\
\text { year }\end{array}$ & Date & $\begin{array}{l}\text { Gage } \\
\text { height } \\
\text { (feet) }\end{array}$ & $\begin{array}{c}\text { Discharge } \\
\text { (cfs) }\end{array}$ \\
\hline 1958 & $\begin{array}{l}\text { May } 3,1958 \\
\text { Aug. } 24,1958\end{array}$ & $\begin{array}{l}10.35 \\
13.86\end{array}$ & $\begin{array}{l}1,140 \\
4,340\end{array}$ & 1961 & $\begin{array}{l}\text { Dec. } 8,1960 \\
\text { Jan. 8, } 1961 \\
\text { Jan. 12, } 1961\end{array}$ & $\begin{array}{l}11.19 \\
10.75 \\
10.40\end{array}$ & $\begin{array}{l}1,590 \\
1,340 \\
1,160\end{array}$ \\
\hline 1959 & $\begin{array}{ll}\text { May } 11, & 1959 \\
\text { June } 24,1959\end{array}$ & $\begin{array}{l}11.38 \\
13.73\end{array}$ & $\begin{array}{l}1,720 \\
4,100\end{array}$ & & $\begin{array}{l}\text { Feb. } 5,1961 \\
\text { Feb. } 16,1961 \\
\text { June } 17,1961\end{array}$ & $\begin{array}{r}10.30 \\
10.48 \\
9.93\end{array}$ & $\begin{array}{r}1,120 \\
1,200 \\
959\end{array}$ \\
\hline 1960 & $\begin{array}{l}\text { Oct. 4, } 1959 \\
\text { Dec. 15, } 1959\end{array}$ & $\begin{array}{l}11.52 \\
10.29\end{array}$ & $\begin{array}{l}1,810 \\
1,120\end{array}$ & \multirow[b]{2}{*}{1962} & June 18,1961 & 11.60 & 1,870 \\
\hline \multirow[t]{2}{*}{1961} & $\begin{array}{l}\text { Oct. } 18,1960 \\
\text { Dec. } 7,1960\end{array}$ & $\begin{array}{l}10.37 \\
10.10\end{array}$ & $\begin{array}{l}1,150 \\
1,030\end{array}$ & & $\begin{array}{l}\text { Nov. } 22,1961 \\
\text { Apr. } 27,1962\end{array}$ & $\begin{array}{l}10.80 \\
12.42\end{array}$ & $\begin{array}{l}1,360 \\
2,580\end{array}$ \\
\hline & & & & $1963^{\circ}$ & Apr. 28,1963 & 4.52 & 89 \\
\hline
\end{tabular}


Location.--Lat $29^{\circ} 46^{\prime} 31^{\prime \prime}$, long $95^{\circ} 23^{\prime} 54^{\prime \prime}$, near right bank at downstream side of pler of Yale Street Bridge, in Houston, Harris County, $80 \mathrm{ft}$ downstream from Texas and New Orleans Railroad Co. bridge, 2.5 miles upstream from Little Wh1teoak Bayou, and 4.1 miles upstream from mouth.

Drainage area. $--84.7 \mathrm{sq} \mathrm{ml}$. During extreme floods when capacity of drainage ditches is exceeded, the drainage area is defined by natural ridge lines and is $92.0 \mathrm{sq} \mathrm{ml}$.

Gage.--Recording. Datum of gage is $4.08 \mathrm{ft}$ below mean sea level, datum of 1929 , unadjusted for ground surface subsidence resulting from heavy ground-water withdrawals.

Stage-discharge relation.--Defined by current-meter measurements .

Historical data.--Flood of DEc. 9, 1935, was highest since at least 1919, information from local resident. Second highest flood occurred May 31, 1929, from information furnished by engineer for Harris County. The drainage area of Whiteoak Bayou is roughly parallel to that of Buffalo Bayou and records indicate that when there is a major flood on Buffalo Bayou there is a major. flood on whiteoak Bayou (see station 8-740).

Remarks.--Base for partial-duration serles, 1,000 cfs. Peak stages and discharges

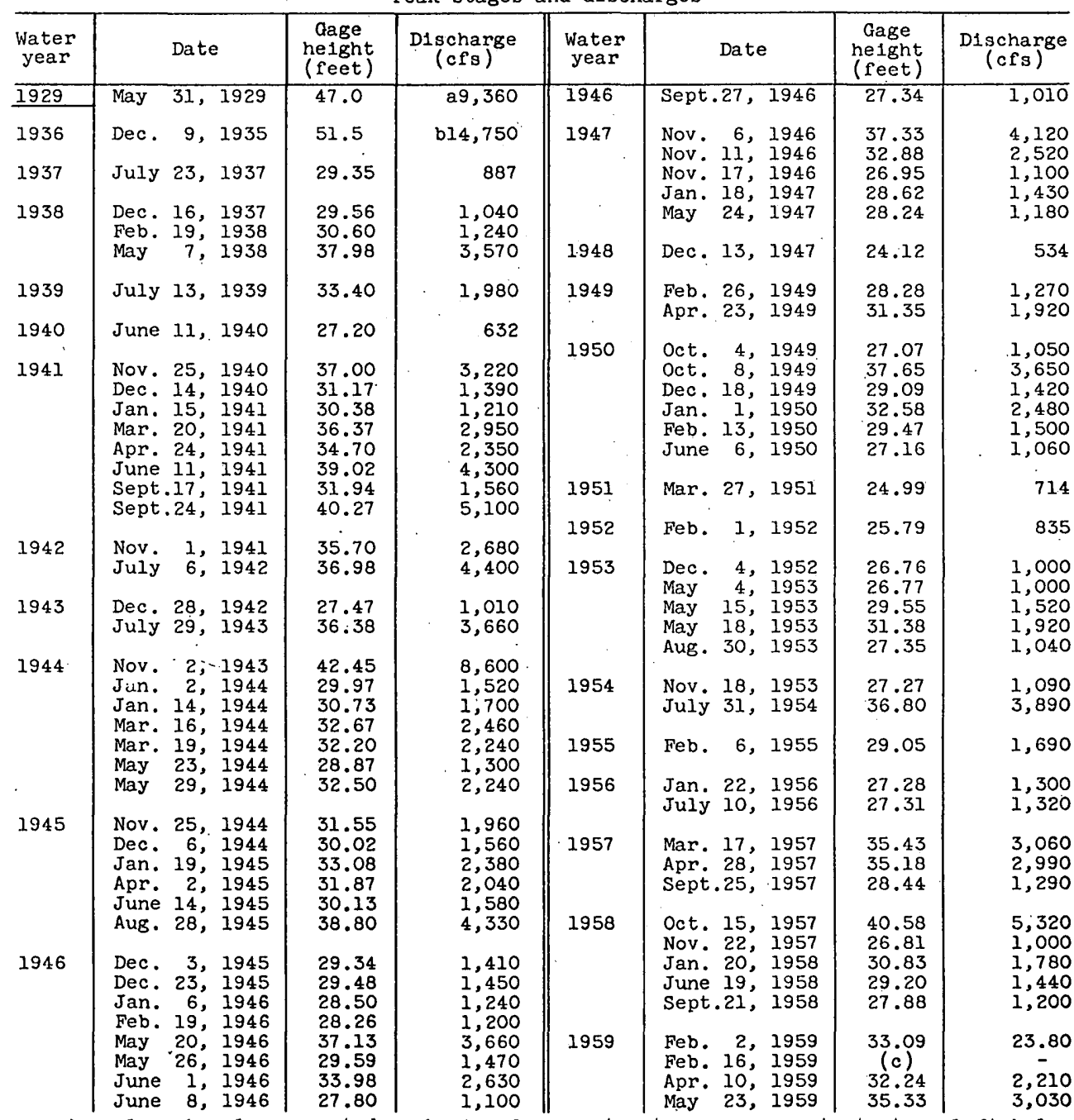
a Annual peak only; computed on bas1s of current-meter measurement at stage 1 ft below crest, furnished by c1ty of Houston.

b Annual peak only, furnished by englneer for llarris County.

c Peak above the base, stage and discharge unknown. 
SAN JACINTO RIVER BASIN

\begin{tabular}{|c|c|c|c|c|c|c|c|}
\hline $\begin{array}{l}\text { Water } \\
\text { year }\end{array}$ & Date & $\begin{array}{c}\text { Qage } \\
\text { nelght } \\
\text { (feet) }\end{array}$ & $\begin{array}{c}\text { D1 gcharge } \\
(\text { cfs })\end{array}$ & $\begin{array}{l}\text { Water } \\
\text { year }\end{array}$ & Date & $\begin{array}{c}\text { Gage } \\
\text { nelght } \\
\text { (feet) }\end{array}$ & $\begin{array}{c}\text { D1scharge } \\
\text { (cfs) }\end{array}$ \\
\hline 1959 & $\begin{array}{l}\text { July 25, } 1959 \\
\text { Aug. 26, } 1959 \\
\text { Sept.22, } 1959 \\
\text { Oct. } 13,1959 \\
\text { Oct. 31, } 1959 \\
\text { Nov. 1, } 1959 \\
\text { Dec. 15, } 1959 \\
\text { Dec. 31, } 1959\end{array}$ & $\begin{array}{l}30.05 \\
39.18 \\
33.72 \\
\\
27.35 \\
28.27 \\
32.92 \\
31.82 \\
28.22\end{array}$ & $\begin{array}{l}1,600 \\
4,510 \\
2,550 \\
1,040 \\
1,290 \\
2,460 \\
2,320 \\
1,260\end{array}$ & 1961 & $\begin{array}{l}\text { Jan. } 7,1961 \\
\text { Feb. 5, } 1961 \\
\text { Feb. 17, } 1961 \\
\text { Feb. 21, } 1961 \\
\text { June 19, } 1961 \\
\text { June } 25,1961 \\
\text { July } 12,1961 \\
\text { Sept.12, } 1961\end{array}$ & $\begin{array}{l}27.27 \\
29.44 \\
39.45 \\
31.80 \\
36.33 \\
29.20 \\
35.49 \\
40.37\end{array}$ & $\begin{array}{l}1,060 \\
1,530 \\
7,380 \\
2,240 \\
4,180 \\
1,480 \\
3,700 \\
5,700\end{array}$ \\
\hline 1961 & $\begin{array}{l}\text { Feb. } 3,1960 \\
\text { Feb. } 21,1960 \\
\text { June 26, } 1960 \\
\text { July } 20,1960 \\
\text { Oct. } 5,1960 \\
\text { Oct. } 19,1960\end{array}$ & $\begin{array}{l}27.45 \\
27.22 \\
40.58 \\
30.08 \\
29.80 \\
32.92\end{array}$ & $\begin{array}{l}1,060 \\
1,020 \\
4,380 \\
1,800 \\
1,640 \\
2,570\end{array}$ & 1962 & $\begin{array}{l}\text { Nov. } 13,1961 \\
\text { Dec. 11, } 1961 \\
\text { Apr. 27, } 1962 \\
\text { May 1, } 1962 \\
\text { June 4, } 1962 \\
\text { June 30, } 1962\end{array}$ & $\begin{array}{l}43.60 \\
28.72 \\
28.22 \\
31.90 \\
38.28 \\
27.58\end{array}$ & $\begin{array}{l}9,000 \\
1,360 \\
1,240 \\
2,270 \\
5,000 \\
1,120\end{array}$ \\
\hline & $\begin{array}{l}\text { Oct. } 29,1960 \\
\text { Nov. } 18,1960 \\
\text { Dec. } 9,1960 \\
\text { Dec. } 31,1960\end{array}$ & $\begin{array}{l}31.50 \\
33.62 \\
30.60 \\
29.62\end{array}$ & $\begin{array}{l}2,150 \\
2,840 \\
1,880 \\
1,590\end{array}$ & 1.963 & $\begin{array}{l}\text { Nov. } 27,1962 \\
\text { Dec. } 24,1962 \\
\text { Jan. 18, } 1963 \\
\text { Feb. 18, } 1963 \\
\text { June } 25,1963 \\
\end{array}$ & $\begin{array}{l}38.48 \\
27.60 \\
30.38 \\
30.40 \\
29.55 \\
\end{array}$ & $\begin{array}{l}5,020 \\
1,120 \\
1,820 \\
1,820 \\
1,590 \\
\end{array}$ \\
\hline
\end{tabular}

8-750. Brays Bayou at Houston, Tex. (12)

Location.--Lat $29^{\circ} 41^{\prime} 49^{\prime \prime}$, long $95^{\circ} 23^{\prime} 43^{\prime \prime}$, at bridge on Main Street, in Houston, Harris County, 1.6 miles upstream from Harris Gully, and 11.6 miles upstream from Buffalo Bayou.

Drainage area. $--89.1 \mathrm{sq} \mathrm{ml}$ prior to Nov. 26,$1959 ; 88.4 \mathrm{sq} \mathrm{mi}$ thereafter. Durlng extreme floods when capacity of drainage ditches is exceeded, the drainage area is defined by natural ridge lines and is $100 \mathrm{sq} \mathrm{ml}$.

Gage.--Recording. At site 0.8 mile upstream at same datum after Nov. 26, 1959. Datum of gage is $3.90 \mathrm{ft}$ below mean sea level, datum of 1929, unadjusted for ground surface subsidence resulting from heavy ground-water withdrawals.

Stage-discharge relation.--Derined by current-meter measurements. Relation affected by channel improvements of July 1923 to. January 1926, flood control work on bayou at various times between 1936 and 1956, and channel lining project that began in 1956 and was st11l incomplete at the end of the 1960 water year.

H1storical data.--Flood in June 1919 was maximum since at least 1911, from information by engineer for city of Houston.

Remarks.--Partial urbanization of the drainage basin as well as channel 1mprovements have changed flood characteristics in recent years. Only annual peaks are shown.

Peak stages and discharges

\begin{tabular}{|c|c|c|c|c|c|c|c|}
\hline $\begin{array}{l}\text { Water } \\
\text { year }\end{array}$ & Date & $\begin{array}{c}\text { Cage } \\
\text { he1ght } \\
\text { (reet) }\end{array}$ & $\begin{array}{c}\text { Discharge } \\
\text { (cfs) }\end{array}$ & $\begin{array}{l}\text { Water } \\
\text { year }\end{array}$ & Date & $\begin{array}{c}\text { Gage } \\
\text { nelght } \\
\text { (feet) }\end{array}$ & $\begin{array}{c}\text { Discharge } \\
\text { (cfs) }\end{array}$ \\
\hline 1919 & June 1919 & 56.0 & - & 1951 & Mar. 28, 1951 & 34.58 & 786 \\
\hline 1922 & March 1922 & 55.3 & - & 1953 & $\begin{array}{l}\text { Feb. } 1,1952 \\
\text { May } 18,1953\end{array}$ & $\begin{array}{l}38.79 \\
42.72\end{array}$ & $\begin{array}{l}1,850 \\
3,580\end{array}$ \\
\hline 1924 & Dec. 23, 1923 & 53.0 & - & $\begin{array}{l}1954 \\
1955\end{array}$ & $\begin{array}{l}\text { Nov. } 18,1953 \\
\text { Feb. } 4,1955\end{array}$ & $\begin{array}{l}43.20 \\
42.38\end{array}$ & $\begin{array}{l}3,680 \\
3,300\end{array}$ \\
\hline 1929 & May 31,1929 & 50.4 & a.11,100 & 1956 & May 2, 1956 & 36.38 & 1,180 \\
\hline $\begin{array}{l}1936 \\
1937 \\
1938\end{array}$ & $\begin{array}{lll}\text { May } 25, & 1936 \\
\text { Dec. } 10,1936 \\
\text { May } 17,1938\end{array}$ & $\begin{array}{l}47.0 \\
34.88 \\
43.27\end{array}$ & $\begin{array}{r}66,600 \\
1,270 \\
4,530\end{array}$ & $\begin{array}{l}1957 \\
1958 \\
1959 \\
1960\end{array}$ & $\begin{array}{l}\text { Mar. 17, } 1957 \\
\text { Oct. 15, } 1957 \\
\text { Apr. 9, } 1959 \\
\text { June 26, } 1960\end{array}$ & $\begin{array}{l}43.65 \\
47.48 \\
43.80 \\
49.72\end{array}$ & $\begin{array}{r}4,660 \\
5,100 \\
7,760 \\
12,600\end{array}$ \\
\hline $\begin{array}{l}1.939 \\
1940\end{array}$ & $\begin{array}{l}\text { July 12, } 1939 \\
\text { Feb. } 17,1940\end{array}$ & $\begin{array}{l}48.02 \\
36.73\end{array}$ & $\begin{array}{l}6,800 \\
1,340\end{array}$ & 1961 & Sept.11, 1961 & 41.62 & 6,320 \\
\hline $\begin{array}{l}1941 \\
1942 \\
1943 \\
1944\end{array}$ & $\begin{array}{l}\text { Sept.24, } 1941 \\
\text { Oct. 31, } 1941 \\
\text { July 29, } 1943 \\
\text { Nov. 2, } 1943\end{array}$ & $\begin{array}{l}47.62 \\
44.58 \\
48.22 \\
50.60\end{array}$ & $\begin{array}{l}6,460 \\
4,590 \\
6,280 \\
8,120\end{array}$ & 1962 & $\begin{array}{l}\text { Nov. } 13,1961 \\
\text { May } 1,1962 \\
\text { June } 4,1962\end{array}$ & $\begin{array}{l}43.00 \\
36.73 \\
42.28\end{array}$ & $\begin{array}{l}7,720 \\
3,700 \\
7,230\end{array}$ \\
\hline 1945 & Aug. 28,1945 & $c 51.70$ & 5,590 & 1963 & Nov. 27,1962 & 36.92 & 4,180 \\
\hline $\begin{array}{l}1946 \\
1947 \\
1948 \\
1949 \\
1950 \\
\end{array}$ & $\begin{array}{l}\text { Sept.27, } 1946 \\
\text { Nov. 5, } 1946 \\
\text { Dec. 13, } 1947 \\
\text { Feb. 26, } 1949 \\
\text { Oct. } 8,1949 \\
\end{array}$ & $\begin{array}{l}48.24 \\
46.25 \\
38.44 \\
42.60 \\
.51 .49 \\
\end{array}$ & $\begin{array}{l}3,880 \\
4,360 \\
1,440 \\
2,340 \\
5,340 \\
\end{array}$ & & $\begin{array}{l}\text { June 22, } 1963 \\
\text { June } 25,1963\end{array}$ & $\begin{array}{l}33.55 \\
35.98\end{array}$ & $\begin{array}{l}3,220 \\
4,740\end{array}$ \\
\hline
\end{tabular}

a From current-meter measurement at Lawndale Avenue Bridge 8 miles downstream, furand Lawndale Ave.

b Maximum for period May 25 to Sept.30, 1936; may have been exceeded during period of no record.

c Occurred at different time than peak discharge. 
Location.--Lat $29^{\circ} 40127^{\prime \prime}$, long $95^{\circ} 17^{\prime} 21^{\prime \prime}$, on left bank at downstream side of brldge on State Highway 35, in southeast section of Houston, Harris County, 5.6 miles upstream from mouth.

Drainage area. $--64.0 \mathrm{sq} \mathrm{ml}$.

Gage.--Recording. Datum of gage is $0.61 \mathrm{ft}$ below mean sea level, datum of I929, adjustment of 1957.

Stage-discharge relation.--Defined by current-meter measurements. Channel was rectlfied January 1957.

Bankfull stage. $--25 \mathrm{ft}$.

Remarks.--Base for partial-duration series, $850 \mathrm{cfs}$.

\begin{tabular}{|c|c|c|c|c|c|c|c|}
\hline $\begin{array}{l}\text { Water } \\
\text { year }\end{array}$ & Date & $\begin{array}{c}\text { aage } \\
\text { he1ght } \\
\text { (reet) }\end{array}$ & $\begin{array}{c}\text { D1scharge } \\
\text { (cfs) }\end{array}$ & $\begin{array}{l}\text { Water } \\
\text { year }\end{array}$ & Date & $\begin{array}{c}\text { Gage } \\
\text { nelght } \\
\text { (feet) }\end{array}$ & $\begin{array}{c}\text { D1scharge } \\
(\mathrm{cfs})\end{array}$ \\
\hline 1953 & $\begin{array}{l}\text { Dec. 22, } 1952 \\
\text { Jan. } 22,1953 \\
\text { Feb. 1, } 1953 \\
\text { Feb. } 24,1953 \\
\text { May 18, } 1953 \\
\text { Aug. 30, } 1953\end{array}$ & $\begin{array}{l}15.75 \\
15.05 \\
14.88 \\
15.07 \\
21.35 \\
19.82\end{array}$ & $\begin{array}{c}900 \\
- \\
- \\
- \\
2,270 \\
1,800\end{array}$ & 1959 & $\begin{array}{l}\text { May } 23,1959 \\
\text { July } 25,1959 \\
\text { Aug. } 23,1959 \\
\text { Aug. } 26,1959 \\
\text { Sept.24, } 1959 \\
\text { oct. } 14,1959\end{array}$ & $\begin{array}{l}17.27 \\
23.91 \\
12.95 \\
21.17 \\
15.70 \\
13.91\end{array}$ & $\begin{array}{r}1,880 \\
3,860 \\
880 \\
3,010 \\
1,480 \\
1,060\end{array}$ \\
\hline 1954 & $\begin{array}{l}\text { Nov. } 19,1953 \\
\text { Dec. } 20,1953\end{array}$ & $\begin{array}{l}18.30 \\
17.05\end{array}$ & $\begin{array}{l}1,410 \\
1,100\end{array}$ & 1960 & $\begin{array}{l}\text { Oct. } 14,1959 \\
\text { Oct. 31, } 1959 \\
\text { Dec. 16, } 1959 \\
\text { Dec. 31, } 1959\end{array}$ & $\begin{array}{l}13.91 \\
16.50 \\
18.82 \\
14.14\end{array}$ & $\begin{array}{l}1,060 \\
1,680 \\
2,290 \\
1,100\end{array}$ \\
\hline 1.955 & Feb. 6,1955 & 21.14 & 2,730 & & $\begin{array}{l}\text { Feb. } 21,1960 \\
\text { June } 26,1960\end{array}$ & $\begin{array}{l}13.34 \\
29.76\end{array}$ & $\begin{array}{r}940 \\
8,030\end{array}$ \\
\hline 1956 & Jan. 31, 1956 & 13.62 & 478 & . & Aug. 24,1960 & 16.80 & 1,750 \\
\hline 1957 & $\begin{array}{l}\text { Mar. 17, } 1957 \\
\text { Mar. 28, } 1957 \\
\text { Apr. 29, } 1957\end{array}$ & $\begin{array}{l}22.12 \\
10.24 \\
18.00\end{array}$ & $\begin{array}{l}4,540 \\
2, \overline{8} 30\end{array}$ & 1961 & $\begin{array}{l}\text { Oct. } 19,1960 \\
\text { Oct. } 29,1960 \\
\text { Dec. } 8,1960 \\
\text { Dec. } 14,1960\end{array}$ & $\begin{array}{l}14.86 \\
15.90 \\
15.29 \\
16.81\end{array}$ & $\begin{array}{l}1,000 \\
1,200 \\
1,080 \\
1,380\end{array}$ \\
\hline 1958 & $\begin{array}{l}\text { Oct. 15, } 1957 \\
\text { Nov. 13, } 1957 \\
\text { Nov. 23, } 1957 \\
\text { Jan. } 20,1958 \\
\text { Jan. } 23,1958 \\
\text { Feb. 23, } 1958 \\
\text { July 2, } 1958 \\
\text { Sept.21, } 1958\end{array}$ & $\begin{array}{l}23.22 \\
12.42 \\
13.9 \\
14.48 \\
12.05 \\
12.9 \\
12.90 \\
18.90\end{array}$ & $\begin{array}{l}5,050 \\
1,070 \\
1,420 \\
1,610 \\
851 \\
1,120 \\
1,120 \\
3,180\end{array}$ & $\therefore$ & $\begin{array}{l}\text { Dec. } 31,1960 \\
\text { Jan. } 7,1961 \\
\text { Feb. 6, } 1961 \\
\text { Feb. 17, } 1961 \\
\text { June 19, } 1961 \\
\text { July 10, } 1961 \\
\text { July 12, } 1961 \\
\text { Sept.12, } 1961\end{array}$ & $\begin{array}{l}18.70 \\
19.36 \\
17.37 \\
14.55 \\
24.28 \\
20.15 \\
20.82 \\
a 28.62\end{array}$ & $\begin{array}{l}1,830 \\
2,030 \\
1,510 \\
948 \\
3,960 \\
2,230 \\
2,440 \\
3,220\end{array}$ \\
\hline 1959 & $\begin{array}{l}\text { Feb. 2, } 1959 \\
\text { Feb. 11, } 1959 \\
\text { Feb. 14, } 1959 \\
\text { Feb. 24, } 1959 \\
\text { Apr. 10, } 1959\end{array}$ & $\begin{array}{l}20.51 \\
14.11 \\
15.67 \\
17.92 \\
23.40\end{array}$ & $\begin{array}{l}3,370 \\
1,300 \\
1,780 \\
2,530 \\
3,690\end{array}$ & 1962 & $\begin{array}{l}\text { Nov. } 13,1961 \\
\text { June } 4,1962 \\
\\
\text { Nov. } 27,1962 \\
\text { Dec. 24, } 1962 \\
\text { Jan. 17, } 1963\end{array}$ & $\begin{array}{l}23.78 \\
20.55 \\
19.80 \\
18.40 \\
22.56\end{array}$ & $\begin{array}{l}3,700 \\
1,920 \\
1,680 \\
1,330 \\
2,690\end{array}$ \\
\hline
\end{tabular}

a Ba.ckwater from tides caused by hurricane Carla.

\section{8-760. Greens Bayou near Houston, Tex:(12)}

Location.--Lat $29^{\circ} 55^{\prime} 05^{\prime \prime}$, long $95^{\circ} 18^{\prime} 24^{\prime \prime}$, on right bank at downstream side of bridge on U.S. Highway 59, 10.5 miles northeast of Houston, Harris County, and 12.0 miles upstream from Halls Bayou.

Drainage area. $--72.7 \mathrm{sq} \mathrm{ml}$.

Gage.--Recording. At site $100 \mathrm{ft}$ upstream prior to Oct. 9, 1958. Datum of gage is $0.66 \mathrm{ft}$ below mean sea level, datum of 1929 , adjustment of 1957 .

Stage-discharge relation.--Defined by current-meter measurements.

Bankfull stage. $--60 \mathrm{ft}$.

Remarks.--Channel was rectified prior to installation of gage. Base for partial-duration series, $700 \mathrm{cfs}$.

\begin{tabular}{|c|c|c|c|c|c|c|c|c|}
\hline $\begin{array}{l}\text { Water } \\
\text { year }\end{array}$ & & Date & $\begin{array}{c}\text { Qage } \\
\text { ne1ght } \\
\text { (feet) }\end{array}$ & $\begin{array}{c}\text { Discharge } \\
\text { (cfs) }\end{array}$ & $\begin{array}{l}\text { Water } \\
\text { year }\end{array}$ & Date & $\begin{array}{c}\text { Gage } \\
\text { helght } \\
\text { (feet) }\end{array}$ & $\begin{array}{c}\text { Discharge } \\
(\mathrm{cfs})\end{array}$ \\
\hline 1953 & $\begin{array}{l}\text { May } \\
\text { May } \\
\text { May } \\
\text { May }\end{array}$ & $\begin{array}{l}3,1953 \\
13,1953 \\
15,1953 \\
18,1953\end{array}$ & $\begin{array}{l}54.65 \\
55.79 \\
55.54 \\
61.38\end{array}$ & $\begin{array}{l}1,090 \\
1,430 \\
1,340 \\
3,280\end{array}$ & $\begin{array}{l}1955 \\
1956\end{array}$ & $\begin{array}{l}\text { Feb. } 4,1955 \\
\text { Oct. } 6,1955 \\
\text { Aug. } 27,1956\end{array}$ & $\begin{array}{l}56.94 \\
53.31 \\
57.09\end{array}$ & $\begin{array}{r}1,740 \\
748 \\
1,400\end{array}$ \\
\hline .1954 & July & 30,1954 & 64.75 & 7,000 & 1957 & Mar. 17,1957 & 59.03 & 1,840 \\
\hline
\end{tabular}


SAN JACINTO RIVER BASIN

Peak stages and d1scharges of Greens Bayou near Houston, Tex.--Cont1nued

\begin{tabular}{|c|c|c|c|c|c|c|c|}
\hline $\begin{array}{l}\text { Water } \\
\text { year }\end{array}$ & Date & $\begin{array}{c}\text { Gage } \\
\text { he1ght } \\
\text { (reet) }\end{array}$ & $\begin{array}{c}\text { D1scharge } \\
\text { (cfs) }\end{array}$ & $\begin{array}{l}\text { Water } \\
\text { year }\end{array}$ & Date & $\begin{array}{c}\text { Gage } \\
\text { he1ght } \\
\text { (feet) }\end{array}$ & $\begin{array}{c}\text { D1 gcharge } \\
(\mathrm{cfs})\end{array}$ \\
\hline $1958^{\circ}$ & $\begin{array}{l}\text { Apr. 29, } 1957 \\
\text { Sept.26, } 1957 \\
\text { Oct. } 16,1957 \\
\text { Nov. 22, } 1957 \\
\text { Jan. 20, } 1958 \\
\end{array}$ & $\begin{array}{l}55.29 \\
57.82 \\
62.82 \\
55.85 \\
59.67\end{array}$ & $\begin{array}{r}860 \\
1,490 \\
3,410 \\
974 \\
2,060 \\
\end{array}$ & \multirow[t]{2}{*}{1961} & \multirow{2}{*}{$\begin{array}{l}\text { Oct. 19, } 1960 \\
\text { Oct. 29, } 1960 \\
\text { Nov..18, } 1960 \\
\text { Dec. 9, } 1960 \\
\text { Dec. } 31,1960 \\
\text { Jan. 7, } 1961 \\
\text { Feb. 17, } 1961 \\
\text { Feb. 21, } 1961 \\
\text { June 19, } 1961 \\
\text { July 12, } 1961 \\
\text { Sept.12, } 1961\end{array}$} & \multirow{2}{*}{$\begin{array}{l}58.94 \\
58.67 \\
61.35 \\
59.53 \\
56.65 \\
56.46 \\
64.09 \\
58.67 \\
61.10 \\
63.25 \\
65.75\end{array}$} & \multirow{2}{*}{$\begin{array}{r}1,100 \\
1,060 \\
1,700 \\
1,560 \\
900 \\
880 \\
4,240 \\
1,360 \\
2,080 \\
3,420 \\
6,120\end{array}$} \\
\hline 1959 & $\begin{array}{l}\text { Feb. 2, } 1959 \\
\text { Apr. } 9,1959 \\
\text { Apr. 12, } 1959 \\
\text { May 11, } 1959 \\
\text { May 23, } 1959 \\
\text { July 25, } 1959 \\
\text { Aug. 27, } 1959\end{array}$ & $\begin{array}{l}57.84 \\
56.98 \\
58.97 \\
58.05 \\
61.32 \\
59.71 \\
60.82\end{array}$ & $\begin{array}{r}1,110 \\
930 \\
1,430 \\
1,160 \\
2,380 \\
1,660 \\
2,090\end{array}$ & & & & \\
\hline 1960 & $\begin{array}{l}\text { Oct. } 14,1959 \\
\text { Dec. } 16,1959 \\
\text { June } 26,27, \\
1960\end{array}$ & $\begin{array}{l}58.47 \\
60.67 \\
63.92\end{array}$ & $\begin{array}{l}1,290 \\
1,520 \\
2,530\end{array}$ & 1963 & $\begin{array}{l}\text { Nov. 27, } 1962 \\
\text { Feb. } 18,1963\end{array}$ & $\begin{array}{l}63.47 \\
58.58\end{array}$ & $\begin{array}{l}3,000 \\
1,040\end{array}$ \\
\hline
\end{tabular}

8-765. Halls Bayou at Houston, Tex. (12)

Location.--Lat $29^{\circ} 51^{\prime} 42^{\prime \prime}$, long $95^{\circ} 20^{\prime} 05^{\prime \prime}$, on right bank at downstream side of bridge on Jensen Drive (formerly U.S. Highway 59), in northeast section of Houston, Harris County, 11.0 miles upstream from mouth.

Drainage area. $--24.7 \mathrm{sq} \mathrm{m} 1$.

Gage.--Recording. Datum of gage 1s $0.66 \mathrm{ft}$ below mean sea level, datum of 1929 , ad jus tment of 1957.

Stage-discharge relation.--Defined by current-meter measurements below 2,100 cf's. Channel was rectified prior to installation of gage. It was rectified again in June 1956, lowering channel about $2 \mathrm{ft}$.

Bankfull stage. $--58 \mathrm{ft}$.

Remarks.--Base for partial-duration series, $300 \mathrm{cfs}$.

\begin{tabular}{|c|c|c|c|c|c|c|c|}
\hline $\begin{array}{l}\text { Water } \\
\text { year }\end{array}$ & Date & $\begin{array}{l}\text { Cage } \\
\text { he1ght } \\
\text { (feet) }\end{array}$ & $\begin{array}{c}\text { Discharge } \\
\text { (cfs) }\end{array}$ & $\begin{array}{l}\text { Water } \\
\text { year }\end{array}$ & Date & $\begin{array}{c}\text { Gage } \\
\text { he1ght } \\
\text { (feet) }\end{array}$ & $\begin{array}{c}\text { Discharge } \\
\text { (cfs) }\end{array}$ \\
\hline 1953 & $\begin{array}{l}\text { Dec. } 4,1952 \\
\text { Dec. } 30,1952 \\
\text { May } 4,1953 \\
\text { May } 13,1953\end{array}$ & $\begin{array}{l}52.74 \\
51.65 \\
52.98 \\
53.42\end{array}$ & $\begin{array}{l}446 \\
310 \\
610 \\
690\end{array}$ & 1959 & $\begin{array}{l}\text { July 20, } 1959 \\
\text { July 25, } 1959 \\
\text { Aug. 26, } 1959\end{array}$ & $\begin{array}{l}53.20 \\
57.61 \\
56.52\end{array}$ & $\begin{array}{r}742 \\
1,810 \\
1,480\end{array}$ \\
\hline & $\begin{array}{ll}\text { May } 15,1953 \\
\text { May } 18,1953\end{array}$ & $\begin{array}{l}54.38 \\
59.05\end{array}$ & 2,410 & 1960 & $\begin{array}{l}\text { Oct. } 14,1959 \\
\text { Dec. } 15,1959 \\
\text { Dec. } 31,1959\end{array}$ & $\begin{array}{l}52.17 \\
52.84 \\
49.77\end{array}$ & $\begin{array}{l}588 \\
678 \\
318\end{array}$ \\
\hline 1954 & $\begin{array}{l}\text { Nov. } 18,1953 \\
\text { Jan. } 10,1954 \\
\text { Jan. } 14,1954\end{array}$ & $\begin{array}{l}53.05 \\
51.98 \\
51.35\end{array}$ & $\begin{array}{r}610 \\
430 \\
343\end{array}$ & & $\begin{array}{l}\text { June } 26,1960 \\
\text { July } 20,1960\end{array}$ & $\begin{array}{l}58.79 \\
53.22\end{array}$ & $\begin{array}{r}2,230 \\
742\end{array}$ \\
\hline & July 30,1954 & 60.65 & 2,020 & 1961 & $\begin{array}{l}\text { Oct. } 19,1960 \\
\text { Oct. } 29,1960\end{array}$ & $\begin{array}{l}51.41 \\
52.63\end{array}$ & $\begin{array}{l}594 \\
850\end{array}$ \\
\hline 1955 & $\begin{array}{l}\text { Jan. } 18,1955 \\
\text { Feb. } 6,1955 \\
\text { Aug. } 8,1955\end{array}$ & $\begin{array}{l}51.95 \\
56.62 \\
55.23\end{array}$ & $\begin{array}{r}430 \\
1,530 \\
1,120\end{array}$ & & $\begin{array}{l}\text { Nov. } 18,1960 \\
\text { Nov. } 20,1960 \\
\text { Dec. } 9,1960 \\
\text { Dec. } 14,1960\end{array}$ & $\begin{array}{l}53.90 \\
49.75 \\
53.45 \\
49.35\end{array}$ & $\begin{array}{r}1,200 \\
340 \\
1,100 \\
300\end{array}$ \\
\hline 1956 & Jan. 22, 1956 & 51.53 & 357 & & $\begin{array}{l}\text { Dec. } 31,1960 \\
\text { Jan. } 7,1961\end{array}$ & $\begin{array}{l}51.77 \\
51.83\end{array}$ & $\begin{array}{l}682 \\
682\end{array}$ \\
\hline 1957 & $\begin{array}{ll}\text { Max. } 17, & 1957 \\
\text { Apr. } 29,1957 \\
\text { Sept.25, } 1957\end{array}$ & $\begin{array}{l}52.14 \\
52.51 \\
50.81\end{array}$ & $\begin{array}{l}572 \\
620 \\
426\end{array}$ & & $\begin{array}{l}\text { Jan. 12, } 1961 \\
\text { Feb. } 5,1961 \\
\text { Feb. 17, } 1961\end{array}$ & $\begin{array}{l}49.81 \\
51.17 \\
56.88 \\
51.48\end{array}$ & $\begin{array}{r}352 \\
562 \\
2,370 \\
610\end{array}$ \\
\hline 1958 & $\begin{array}{l}\text { Oct. } 15,1957 \\
\text { Nov. } 22,1957 \\
\text { Jan. } 20,1958 \\
\text { Jan. 23, } 1958 \\
\text { Feb. 22, } 1958 \\
\text { Sept.20, } 1958\end{array}$ & $\begin{array}{l}57.09 \\
51.73 \\
53.43 \\
49.55 \\
50.03 \\
-\end{array}$ & $\begin{array}{r}1,280 \\
525 \\
732 \\
324 \\
344 \\
8.560\end{array}$ & & $\begin{array}{l}\text { June } 12,1961 \\
\text { June } 19,1961 \\
\text { July } 3,1961 \\
\text { July } 9,1961 \\
\text { July 12, } 1961 \\
\text { July 17, } 1961 \\
\text { Sept.12, } 1961\end{array}$ & $\begin{array}{l}49.68 \\
57.48 \\
52.96 \\
52.88 \\
58.29 \\
49.63 \\
60.50\end{array}$ & $\begin{array}{r}310 \\
2,050 \\
790 \\
772 \\
2,370 \\
300 \\
400\end{array}$ \\
\hline 1959 & $\begin{array}{l}\text { Feb. } 2,1959 \\
\text { Feb. } 11,1959 \\
\text { Feb. } 15,1959 \\
\text { Feb. } 25,1959 \\
\text { Apr. 11, } 1959 \\
\text { May 11, } 1959\end{array}$ & $\begin{array}{l}56.04 \\
50.36 \\
(\mathrm{~b}) \\
(\mathrm{b}) \\
54.27 \\
54.86\end{array}$ & $\begin{array}{c}1,340 \\
378 \\
- \\
- \\
950 \\
1,080\end{array}$ & 1962 & $\begin{array}{l}\text { Sept.12, } 1901 \\
\text { Nov. } 13,1961 \\
\text { Dec. 11, } 1961 \\
\text { May 1, } 1962 \\
\text { June } 4,1962\end{array}$ & $\begin{array}{l}60.50 \\
58.28 \\
52.57 \\
52.75 \\
53.58\end{array}$ & $\begin{array}{r}2,540 \\
772 \\
754 \\
912\end{array}$ \\
\hline & $\begin{array}{lll}\text { May 23, } 1959 \\
\text { July } 6,1959\end{array}$ & $\begin{array}{l}58.10 \\
51.71\end{array}$ & $\begin{array}{r}1,980 \\
524\end{array}$ & 1963 & $\begin{array}{l}\text { Nov. } 27,1962 \\
\text { Jan. } 17,1963 \\
\text { Feb. } 18,1963\end{array}$ & $\begin{array}{l}57.02 \\
51.95 \\
52.65\end{array}$ & $\begin{array}{r}1,870 \\
610 \\
718\end{array}$ \\
\hline
\end{tabular}

b Peak above base; stage and discharge unknown. 
8-770. Clear Creek near Pearland, Tex.(12)

Location. --Lat $29^{\circ} 35^{\prime} 50^{\prime \prime}$, long $95^{\circ} 17^{\prime} 12^{\prime \prime}$, at bridge on State Highway $35,0.7$ mile downstream from Gulf, Colorado and Santa Fe Ra1lway bridge, 1.2 miles upstream from Hickory Slough, 2.3 miles north of Pearland, Brazoria County, and about 30 miles upstream from Clear Lake.

Drainage area.--38.4 sq mi, planimetered by Harris County Flood Control District from survey by Corps of Engineers in 1943. Drainage area not applicable for low flows: a large area of rice land above station is irrigated with water from the Brazos River; also, drainage ditches and canals used by irrigators are changed at times, thereby changing the drainage area.

Gage.--Nonrecording prior to June 9, 1948; recording thereafter. At datum $5.80 \mathrm{ft}$ higher prior to Apr. 23, 1952. Datum of gage is $29.29 \mathrm{ft}$ above mean sea level, datum of 1929, adjustment of 1957.

Stage-discharge relation.--Defined by current-meter measurements.

Bankfull stage. $--14 \mathrm{ft}$.

Historical data.--Flood information begins in February 1932 , when flood reached a stage of $17.8 \mathrm{ft}$, present datum, from information by State Highway Department. U.S. Weather Bureau records indicate that the rainfall during the tropical storm of August 1945 was greater than that of February 1932. State Highway information is that the water did not flow over the road (elevation, about $20.0 \mathrm{ft}$, present datum).

Remarks.--Because of channel rectification in 1952, there is no relation between flood peaks prior to April 1952 and subsequent flood peaks. Base for partial-duration series, $600 \cdot \mathrm{cfs}$.

Peak stages and discharges

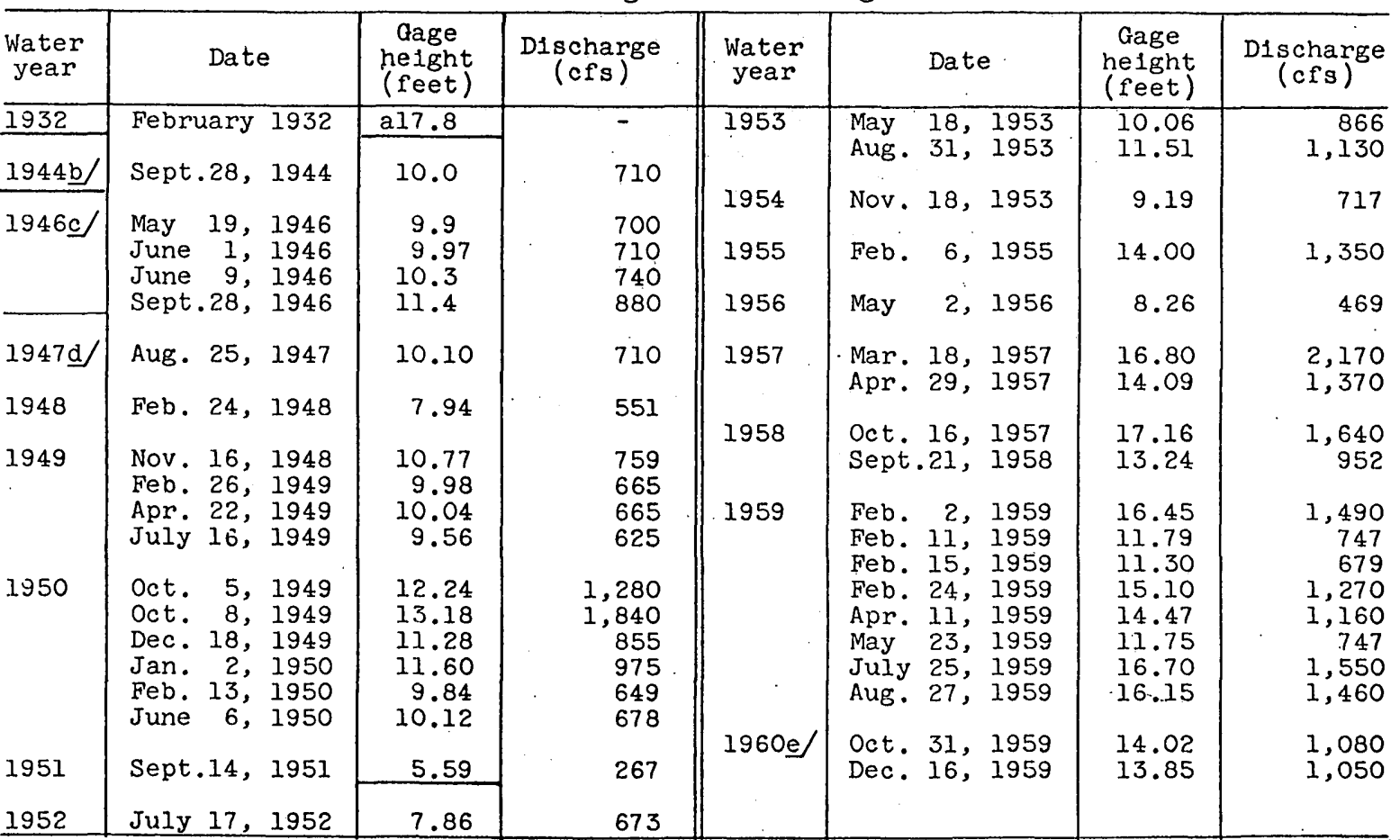

a Present datum.

b Perlod July 28 to Sept. $30,1944$.

c Perlod Mar. 4 to Sept. 30, 1946.

d Perlods October 1946, April to September 1947.

e Period Oct. 1 to Dec. 31, 1959. 
Location.--Lat $29^{\circ} 22^{\prime} 10^{\prime \prime}$, long $95^{\circ} 19^{\prime} 20^{\prime \prime}$, on right bank $800 \mathrm{ft}$ downstream from bridge on Farm Road 1462 and 5.9 miles southwest of Alvin, Brazorla County.

Dra1nage area. $--88.1 \mathrm{sq} \mathrm{m}$.

Gage.--Nonrecording prior to June 12, 1952; recording thereafter, except nonrecording Feb.. 10, 1958, to May 3, 1959. At sites 1,360, 1,400, and $900 \mathrm{ft}$ upstream on old channel at datum $3.00 \mathrm{ft}$ h1gher prior to May 4, 1959. Datum of gage is $10.31 \mathrm{ft}$ above mean sea level, datum of 1929, Houston supplementary adjustment of 1943.

Stage-discharge relation.--Defined by current-meter measurements below 3,300 cfs and extended above by logarithmic plotting. Relationship affected by channel rectification in summer of 1955 and in October 1957.

Bankfull stage. $--15 \mathrm{ft}$.

Historical data.--Flood of July 14, 1939, was highest known in recent years, from information by local residents. U.S. Weather Bureau records show very heavy rains in the area in October 1913 and August 1915.

Remarks.--Records prior to Jan. 14, 1947, are for low flow only. Base for partial-duration series, $800 \mathrm{cfs}$.

Peak stages and discharges

\begin{tabular}{|c|c|c|c|c|c|c|c|}
\hline $\begin{array}{l}\text { Water } \\
\text { year }\end{array}$ & Date & $\begin{array}{c}\text { Qage } \\
\text { helght } \\
\text { (feet) }\end{array}$ & $\begin{array}{c}\text { D1scharge } \\
\text { (cfs) }\end{array}$ & $\begin{array}{l}\text { Water } \\
\text { year }\end{array}$ & Date & $\begin{array}{c}\text { Gage } \\
\text { he1ght } \\
\text { (feet) }\end{array}$ & $\begin{array}{c}\text { D1scharge } \\
\text { (cfs) }\end{array}$ \\
\hline 1939 & July 14,1939 & 19.9 & - & 1057 & $\operatorname{Man}$ & & \\
\hline 1946 & $\begin{array}{lr}\text { May } 22, & 1946 \\
\text { June } 3, & 1946 \\
\text { June } 10, & 1946 \\
\text { July } 7, & 1946 \\
\text { Sept.30, } 1946\end{array}$ & $\begin{array}{c}(a) \\
\text { b15.72 } \\
\left(\begin{array}{l}a \\
a \\
a\end{array}\right)\end{array}$ & $\begin{array}{l}- \\
1,190 \\
- \\
-\end{array}$ & 195 & $\begin{array}{l}\text { Mar. } 21,1957 \\
\text { Apr. } 30,1957 \\
\text { June } 3,1957 \\
\text { June } 7,1957\end{array}$ & $\begin{array}{r}11.56 \\
12.97 \\
9.15 \\
11.28\end{array}$ & $\begin{array}{r}4,200 \\
1,270 \\
1,580 \\
878 \\
1,210\end{array}$ \\
\hline 1947 & $\begin{array}{l}\text { Nov. } 8,1946 \\
\text { Nov. } 18,1946 \\
\text { Nov. } 30,1946 \\
\text { Aug. } 26,1947\end{array}$ & $\left.\begin{array}{l}a \\
a \\
a\end{array}\right\}$ & $\begin{array}{c}- \\
- \\
1,210\end{array}$ & .1958 & $\begin{array}{l}\text { Oct. } 16,1957 \\
\text { Nov. } 14,1957 \\
\text { Nov. } 23,1957 \\
\text { Jan. } 20,1958 \\
\text { Jan. } 24,1958 \\
\end{array}$ & $\begin{array}{r}13.25 \\
6.18 \\
10.12 \\
7.33 \\
.7 .50\end{array}$ & $\begin{array}{r}4,100 \\
906 \\
1,830 \\
990 \\
1,040 \\
\end{array}$ \\
\hline 1948 & Feb. 25,1948 & 14.76 & 1,080 & 1959 & Feb. 25, 1959 & 15.14 & 2,130 \\
\hline 1949 & $\begin{array}{l}\text { Feb. } 27,1949 \\
\text { Apr. } 23,1949\end{array}$ & $\begin{array}{l}12.96 \\
12.99\end{array}$ & $\begin{array}{l}980 \\
980\end{array}$ & & $\begin{array}{ll}\text { May } 23, & 1959 \\
\text { July } 26,1959 \\
\text { Aug. } 27,1959\end{array}$ & $\begin{array}{r}9.12 \\
18.88 \\
19.03\end{array}$ & $\begin{array}{r}848 \\
2,770 \\
3,370\end{array}$ \\
\hline 1950 & $\begin{array}{l}\text { Oct. } 8,1949 \\
\text { Dec. } 12,1949 \\
\text { Dec. 18, } 1949 \\
\text { Jan. 12, } 1950 \\
\text { Feb. 14, } 1950\end{array}$ & $\begin{array}{l}18.80 \\
11.97 \\
16.60 \\
12.10 \\
15.76\end{array}$ & $\begin{array}{r}7,400 \\
846 \\
2,520 \\
863 \\
.1,700\end{array}$ & 1960 & $\begin{array}{l}\text { Nov. } 1,1959 \\
\text { Dec: } 16,1959 \\
\text { Jan. } 1,1960 \\
\text { Feb. 22, } 1960 \\
\text { June 27, } 1960\end{array}$ & $\begin{array}{r}17.72 \\
13.81 \\
8.90 \\
11.57 \\
18.46\end{array}$ & $\begin{array}{r}2,850 \\
1,820 \\
812 \\
1,330 \\
2,920\end{array}$ \\
\hline 1951 & Sept.14, 1951 & 12.66 & 935 & 1961 & $\begin{array}{l}\text { Dec. 9, } 1960 \\
\text { Dec. 15, } 1960\end{array}$ & & $\begin{array}{r}938 \\
1,290\end{array}$ \\
\hline 1952 & $\begin{array}{l}\text { Apr. 1, } 1952 \\
\text { Apr. 23, } 1952 \\
\text { May } 29,1952\end{array}$ & $\begin{array}{l}16.33 \\
16.30 \\
16.25\end{array}$ & $\begin{array}{l}2,250 \\
2,200 \\
2,150\end{array}$ & & $\begin{array}{l}\text { Dec. } 29,1960 \\
\text { Jan. } 1,1961 \\
\text { Jan. } 8,1961 \\
\text { Feb. } 6,1961\end{array}$ & $\begin{array}{l}12.37 \\
13.82 \\
15.84 \\
10.38\end{array}$ & $\begin{array}{r}1,390 \\
1,750 \\
2,300 \\
992\end{array}$ \\
\hline 1953 & $\begin{array}{l}\text { May } 19,1953 \\
\text { June } 30,1953 \\
\text { Aug. } 31,1953\end{array}$ & $\begin{array}{l}15.94 \\
13.15 \\
16.69\end{array}$ & $\begin{array}{l}1,860 \\
1,010 \\
2,660\end{array}$ & ! & $\begin{array}{l}\text { June 19, } 1961 \\
\text { July } 12,1961 \\
\text { Sept.13, } 1961\end{array}$ & $\begin{array}{l}20.10 \\
19.60 \\
19.46\end{array}$ & $\begin{array}{l}3,970 \\
3,510 \\
3,460\end{array}$ \\
\hline 1954 & $\begin{array}{l}\text { Nov. } 19,1953 \\
\text { Dec. } 21,1953\end{array}$ & 16.9 & $\begin{array}{r}3,010 \\
863\end{array}$ & 1962 & Nov. 14,1961 & 18.88 & 3,050 \\
\hline 1955 & Feb. 7,195 & $\begin{array}{l}11.10 \\
15.97\end{array}$ & 1,860 & 1963 & $\begin{array}{l}\text { Nov. } 27,1962 \\
\text { Dec. } 3,1962\end{array}$ & $\begin{array}{l}11.98 \\
10.07\end{array}$ & $\begin{array}{r}1,090 \\
806\end{array}$ \\
\hline 1956 & 2,1956 & 3.72 & 247 & & $\begin{array}{l}\text { Dec. } 24,1962 \\
\text { Dec. } 29,1962 \\
\text { June } 21,1963 \\
\text { June 26, } 1963\end{array}$ & $\begin{array}{r}14.38 \\
9.60 \\
11.61 \\
10.78\end{array}$ & $\begin{array}{r}1,810 \\
888 \\
1,030 \\
906 \\
\end{array}$ \\
\hline
\end{tabular}

a Peak above the base of 800 cfs probably occurred.

b Maximum for per1od Mar. 5 to Sept.30, 1946; may have been exceeded during per1od of no record. 
8-809.33. Unnamed tributary (watershed 1) of Duck Creek

near Spur, Tex. (25)

Location.--Lat $33^{\circ} 28^{\prime}$, long $100^{\circ} 53^{\prime}, 1$ mile west of Spur, Dickens County.

Dra1nage area. $--0.0180 \mathrm{sq} \mathrm{ml}$.

Gage.--Recording.

Remarks.--Records furn1shed by U.S. Department of Agriculture, Agricultural

Research Service. Only annual (calendar year) peaks are shown.

\begin{tabular}{|c|c|c|c|c|c|c|c|}
\hline $\begin{array}{l}\text { Calen- } \\
\text { dar } \\
\text { year }\end{array}$ & Date & $\begin{array}{c}\text { Cage } \\
\text { helght } \\
\text { (feet) }\end{array}$ & $\begin{array}{c}\text { D1scharge } \\
(\text { (cfs) }\end{array}$ & $\begin{array}{l}\text { Calen- } \\
\vdots \text { dar } \\
\text { year }\end{array}$ & Date & $\begin{array}{c}\text { Gage } \\
\text { he1ght } \\
\text { (feet) }\end{array}$ & $\begin{array}{c}\text { D1scharge } \\
(\mathrm{cfs})\end{array}$ \\
\hline $\begin{array}{l}1927 \\
1928 \\
1929 \\
1930\end{array}$ & $\begin{array}{ll}\text { June } & 1,1927 \\
\text { Aug. } & 4,1928 \\
\text { Sept. } & 8,1929 \\
\text { Aug. } & 7,1930\end{array}$ & $\begin{array}{l}\overline{-} \\
\bar{z}\end{array}$ & $\begin{array}{r}4.5 \\
5: 7 \\
14 \\
2.4\end{array}$ & $\begin{array}{l}1937 \\
1938 \\
1939 \\
1940\end{array}$ & $\begin{array}{lr}\text { Aug. } 24, & 1937 \\
\text { June } 25, & 1938 \\
\text { Oct. } 8, & 1939 \\
\text { Nov. } 24, & 1940\end{array}$ & $\begin{array}{l}- \\
- \\
-\end{array}$ & $\begin{array}{l}7.1 \\
2.9 \\
1.0 \\
3.1\end{array}$ \\
\hline $\begin{array}{l}1931 \\
1932 \\
1933 \\
1934 \\
1935 \\
1936\end{array}$ & $\begin{array}{l}\text { May } 25,1931 \\
\text { June } 20,1932 \\
\text { Aug. 2, } 1933 \\
\text { Sept.15, } 1934 \\
\text { June } 7,1935 \\
\text { Sept.20, } 1936\end{array}$ & $\begin{array}{l}- \\
- \\
- \\
-\end{array}$ & $\begin{array}{l}13^{.3} \\
1.5 \\
2.8 \\
7.6 \\
-\end{array}$ & $\begin{array}{l}1941 \\
1942 \\
1943 \\
1944 \\
1945\end{array}$ & $\begin{array}{l}\text { Sept.17, } 1941 \\
\text { June 14, } 1942 \\
\text { July 3, } 1943 \\
\text { June 25, } 1944 \\
\text { July 10, } 1945\end{array}$ & $\begin{array}{l}- \\
- \\
-\end{array}$ & $\begin{array}{r}10 \\
8.0 \\
1.7 \\
4.9\end{array}$ \\
\hline
\end{tabular}

8-809.35. Unnamed tributary (watershed 2) of Duck Creek near Spur, Tex.(25) Location.--Lat $33^{\circ} 28^{\prime}$, long $100^{\circ} 53^{\prime}, 1$ mile west of Spur, Dickens County.

Drainage area. $--0.0147 \mathrm{sq} \mathrm{ml}$.

Gage. --Recording.

Remarks.--Records furnished by U.S. Department of Agriculture, Agricultural Research Service. Only annual (calendar year) peaks are shown.

\begin{tabular}{|c|c|c|c|c|c|c|c|}
\hline $\begin{array}{l}\text { Calen- } \\
\text { dar } \\
\text { year }\end{array}$ & Date & $\begin{array}{c}\text { Gage } \\
\text { helght } \\
\text { (feet) }\end{array}$ & $\begin{array}{c}\text { Discharge } \\
\text { (cfs). }\end{array}$ & $\begin{array}{l}\text { Calen- } \\
\text { dar } \\
\text { year }\end{array}$ & Date & $\begin{array}{c}\text { Gage } \\
\text { helght } \\
\text { (feet) }\end{array}$ & $\begin{array}{l}\text { D1scharge } \\
\text { (cfs) }\end{array}$ \\
\hline $\begin{array}{l}1927 \\
1928 \\
1929 \\
1930\end{array}$ & $\begin{array}{l}\text { June } 1,1927 \\
\text { May 18, } 1928 \\
\text { Sept. } 8,1929 \\
\text { Aug. } 7,1930\end{array}$ & $\overline{-}$ & $\begin{array}{l}1.6 \\
3.7 \\
6.2 \\
2.2\end{array}$ & $\begin{array}{l}1937 \\
1938 \\
1939 \\
1940\end{array}$ & $\begin{array}{l}\text { Aug. } 24,1937 . \\
\text { July } 21,1938 \\
\text { Oct. } 8,1939 \\
\text { Aug. } 17,1940\end{array}$ & $\begin{array}{l}- \\
- \\
-\end{array}$ & $\begin{array}{l}9.4 \\
8.5 \\
1.9 \\
4.3\end{array}$ \\
\hline $\begin{array}{l}1931 \\
1932\end{array}$ & $\begin{array}{ll}\text { May } 25, & 1931 \\
\text { June } 20, & 1932\end{array}$ & - & 1.8 & 1941 & June 15,1941 & - & 9.9 \\
\hline $\begin{array}{l}1933 \\
1934 \\
1935\end{array}$ & $\begin{array}{l}\text { Aug. 2, } 1933 \\
\text { Sept.15, } 1934 \\
\text { May 17, } 1935\end{array}$ & $\overrightarrow{-}$ & $\begin{array}{l}2.9 \\
4.6 \\
5.1\end{array}$ & $\begin{array}{l}1943 \\
1944 \\
1945\end{array}$ & $\begin{array}{lrl}\text { July } & 3, & 1943 \\
\text { June } & 25, & 1944 \\
\text { June } & 4, & 1945\end{array}$ & - & $\begin{array}{l}9.4 \\
6.2 \\
9.9\end{array}$ \\
\hline 1936 & Sept.20, 1936 & - & 13 & & & & \\
\hline
\end{tabular}

8-809.37. Unnamed tributary (watershed 3) of Duck Creek near Spur, Tex. (25) Location.--Lat $33^{\circ} 28^{\prime}$, long $100^{\circ} 53^{\prime}, 1$ mile west of Spur, Dickens County. Drainage area. $-0.0183 \mathrm{sq} \mathrm{ml}$.

Gage.--Recording.

Remarks.--Records furnished by U.S. Department of Agriculture, Agricultural

Research Service. OnIy annual (calendar year) peaks are shown.

Peak stages and discharges

\begin{tabular}{|c|c|c|c|c|c|c|c|}
\hline $\begin{array}{l}\text { Calen- } \\
\text { dar } \\
\text { year }\end{array}$ & Date & $\begin{array}{c}\text { Gage } \\
\text { helght } \\
\text { (feet) }\end{array}$ & $\begin{array}{c}\text { D1scharge } \\
\text { (cfs) }\end{array}$ & $\begin{array}{l}\text { Calen- } \\
\text { dar } \\
\text { year }\end{array}$ & Date & $\begin{array}{c}\text { Gage } \\
\text { height } \\
\text { (feet) }\end{array}$ & $\begin{array}{c}\text { D1scharge } \\
(\mathrm{cfs})\end{array}$ \\
\hline $\begin{array}{l}1927 \\
1928 \\
1929 \\
1930\end{array}$ & $\begin{array}{l}\text { June } 1,1927 \\
\text { May } 18,1928 \\
\text { Sept. } 8,1929 \\
\text { Dec. } 4,1930\end{array}$ & $\begin{array}{l}- \\
- \\
-\end{array}$ & $\begin{array}{r}2.7 \\
5.1 \\
11 \\
1.5\end{array}$ & $\begin{array}{l}1937 \\
1938 \\
1939 \\
1940\end{array}$ & $\begin{array}{l}\text { Aug. } 24,1937 \\
\text { Ju1y } 22,1938 \\
\text { Oct. } 8,1939 \\
\text { Nov. } 25,1940\end{array}$ & $\begin{array}{l}- \\
- \\
-\end{array}$ & $\begin{array}{r}6.6 \\
6.8 \\
.8 \\
.4\end{array}$ \\
\hline $\begin{array}{l}1931 \\
1932 \\
1933 \\
1934 \\
1935\end{array}$ & $\begin{array}{ll}\text { May } 25, & 1931 \\
\text { June } 20, & 1932 \\
\text { May } 24, & 1933 \\
\text { Sept.15, } & 1934 \\
\text { May } 17, & 1935\end{array}$ & $\begin{array}{l}- \\
- \\
-\end{array}$ & $\begin{array}{r}.6 \\
8.5 \\
.7 \\
4.4\end{array}$ & $\begin{array}{l}1941 \\
1942 \\
1943 \\
1944\end{array}$ & $\begin{array}{l}\text { Sept.17, } 1941 \\
\text { Sept.19, } 1942 \text {, } \\
\text { Oct. 17, } 1942 \\
\text { July 3, } 1943 \\
\text { June 25, } 1944\end{array}$ & $\tilde{-}$ & $\begin{array}{l}5.9 \\
(\text { a) } \\
(\text { a })\end{array}$ \\
\hline 1936 & Sept.20, 1936 & - & 13 & & & & \\
\hline
\end{tabular}

a Less than $0.1 \mathrm{cfs}$. 
8-809.39. Unnamed tributary (watershed 5) of Duck Creek near Spur, Tex.(25) . Location.--Lat $33^{\circ} 28^{\prime}$, long $100^{\circ} 53^{\prime}, 1 \mathrm{mlle}$ west of Spur, Dickens County.

Dra1nage area. $--0.0091 \mathrm{sq} \mathrm{ml} ; 0.0086 \mathrm{sq} \mathrm{ml}$ prior to Jan. 1,1936 .

Gage.--Recording.

Remarks.--Records furnished by U.S. Department of Agriculture, Agricultural Research Service. Only annual (calendar year) peaks are shown.

\begin{tabular}{|c|c|c|c|c|c|c|c|}
\hline $\begin{array}{l}\text { Calen- } \\
\text { dar } \\
\text { year }\end{array}$ & Date & $\begin{array}{c}\text { Cage } \\
\text { helght } \\
\text { (feet) }\end{array}$ & $\begin{array}{c}\text { D1scharge } \\
\text { (cfs) }\end{array}$ & $\begin{array}{l}\text { Calen- } \\
\text { dar } \\
\text { year }\end{array}$ & Date & $\begin{array}{c}\text { Gage } \\
\text { ne1ght } \\
\text { (feet) }\end{array}$ & $\begin{array}{c}\text { Discharge } \\
\text { (cfs) }\end{array}$ \\
\hline $\begin{array}{l}1927 \\
1928 \\
1929 \\
1930\end{array}$ & $\begin{array}{lll}\text { June } & 1,1927 \\
\text { Aug. } & 4,1928 \\
\text { Sept. } & 9,1929 \\
\text { Aug. } & 7,1930\end{array}$ & $\overline{-}$ & $\begin{array}{r}4.0 \\
5.6 \\
12 \\
3.2 \\
\end{array}$ & $\begin{array}{l}1936 \\
1937 \\
1938 \\
1939 \\
1940\end{array}$ & $\begin{array}{l}\text { Sept.20, } 1936 \\
\text { Aug. 24, } 1937 \\
\text { July } 22,1938 \\
\text { Oct. } 8,1939 \\
\text { Aug. } 17,1940\end{array}$ & $\begin{array}{l}- \\
- \\
- \\
-\end{array}$ & $\begin{array}{l}8.7 \\
4.0 \\
2.7 \\
.1 \\
2.0\end{array}$ \\
\hline $\begin{array}{l}1931 \\
1932 \\
1933 \\
1934 \\
1935 \\
\end{array}$ & $\begin{array}{lll}\text { May } 25, & 1931 \\
\text { June } 20, & 1932 \\
\text { June } 24, & 1933 \\
\text { Sept.15, } & 1934 \\
\text { May } 17, & 1935 \\
\end{array}$ & $\begin{array}{l}- \\
\overline{-} \\
- \\
\end{array}$ & $\begin{array}{r}2.2 \\
11 . \\
3.1 \\
8.0 \\
5.8 \\
\end{array}$ & $\begin{array}{l}1941 \\
1942 \\
1943 \\
1944 \\
\end{array}$ & $\begin{array}{l}\text { Sept.17, } 1941 \\
\text { Sept.19, 1942 } \\
\text { July 3, 1943 } \\
\text { June 25, 1944 }\end{array}$ & $\begin{array}{l}- \\
- \\
-\end{array}$ & $\begin{array}{l}5.1 \\
2.2 \\
5.0 \\
2.0 \\
\end{array}$ \\
\hline
\end{tabular}

\section{8-809.41. Unnamed tributary (watershed 6) of Duck Creek} near Spur, Tex. (25)

Location.--Lat $33^{\circ} 28^{\prime}$, long $100^{\circ} 53^{\prime}, 1$ mile west of Spur, Dickens County. Drainage area. $-0.0083 \mathrm{sq} \mathrm{ml} ; 0.0094 \mathrm{sq} \mathrm{ml}$ prior to spring 1936 .

Gage.--Recording.

Remarks.--Records furnished by U.S. Department of Agriculture, Agricultural

Research Service. Only annual (calendar year) peaks are shown.

\begin{tabular}{|c|c|c|c|c|c|c|c|}
\hline \multicolumn{8}{|c|}{ Peak stages and discharges } \\
\hline $\begin{array}{l}\begin{array}{c}\text { Calen- } \\
\text { dar } \\
\text { year }\end{array} \\
\end{array}$ & Date & $\begin{array}{c}\text { Gage } \\
\text { ne1ght } \\
\text { (feet) }\end{array}$ & $\begin{array}{c}\text { D1scharge } \\
(\text { cfs })\end{array}$ & $\begin{array}{l}\text { Calen- } \\
\text { dar } \\
\text { year }\end{array}$ & Date & $\begin{array}{c}\text { Gage } \\
\text { he1ght } \\
\text { (feet) }\end{array}$ & $\begin{array}{c}\text { Discharge } \\
(\mathrm{Cr})\end{array}$ \\
\hline $\begin{array}{l}1927 \\
1928 \\
1929\end{array}$ & $\begin{array}{l}\text { June } 1,1927 \\
\text { May } 18,1928, \\
\text { Aug. } 4,1928 \\
\text { Sent }\end{array}$ & - & $\begin{array}{r}0.5 \\
.9\end{array}$ & $\begin{array}{r}1937 \\
1938 \\
1939\end{array}$ & $\begin{array}{ll}\text { Aug. } & 21,1937 \\
\text { July } 22, & 1938 \\
\text { Oct. } & 8,1939\end{array}$ & $=$ & $\begin{array}{l}6.3 \\
8.0 \\
1.4\end{array}$ \\
\hline $\begin{array}{l}1929 \\
1930\end{array}$ & $\begin{array}{l}\text { Sept. } 8,1929 \\
\text { Dec. } 4,1930\end{array}$ & 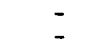 & $\begin{array}{r}1.8 \\
.7\end{array}$ & 1940 & Aug. 17,1940 & - & 6.8 \\
\hline 1931 & June 29,1931 & - & .1 & $\begin{array}{l}1941 \\
1942 \\
1943\end{array}$ & $\begin{array}{ll}\text { Apr. } 29, & 1941 \\
\text { June } 15, & 1942\end{array}$ & $\overline{-}$ & $\begin{array}{l}6.4 \\
2.7\end{array}$ \\
\hline $\begin{array}{l}1933 \\
1934 \\
1935 \\
\end{array}$ & $\begin{array}{l}\text { Aug. } 2,1933 \\
\text { June } 23,1935\end{array}$ & $\overline{-}$ & $\begin{array}{l}.2 \\
0.8 \\
1.8\end{array}$ & $\begin{array}{l}1943 \\
1944 \\
1945\end{array}$ & $\begin{array}{l}\text { July } 3,1943 \\
\text { June } 25,1944 \\
\text { June } 4,1945\end{array}$ & $\overline{-}$ & $\begin{array}{l}- \\
5.2 \\
6.2\end{array}$ \\
\hline
\end{tabular}

8-809.43. Unnamed tributary (watershed 11) of Duck Creek near Spur, Tex. (25)

Location.--Lat $33^{\circ} 28^{\prime}$, long $100^{\circ} 53^{\prime}$, 1 mile west of Spur, Dickens County.

Drainage area. $-0.0136 \mathrm{sq} \mathrm{ml}$.

Gage.--Recording.

Remarks.--Records furn1shed by U.S. Department of Agriculture, Agricultural

Research Service. Only annual (calendar year) peaks are shown.

Peak stages and discharges

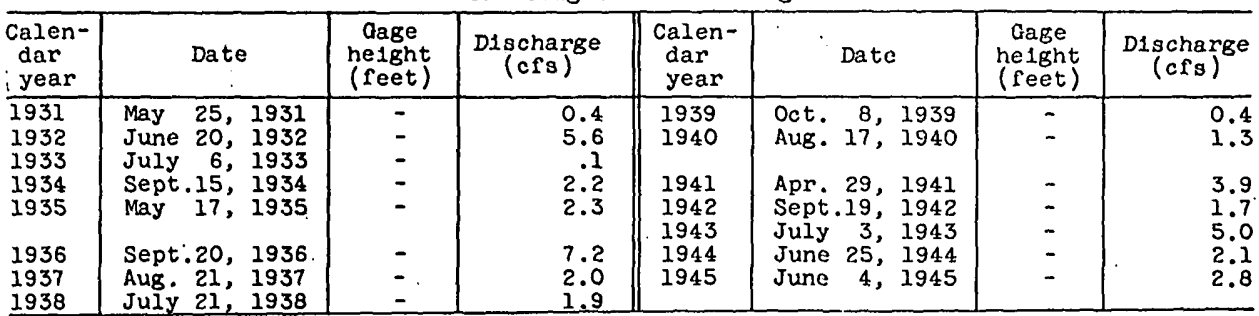




\section{8-809.45. Unnamed tributary (watershed 12) of Duck Creek near Spur, Tex. (25)}

Location.--Lat $33^{\circ} 28^{\prime}$, long $100^{\circ} 53^{\prime}, 1$ mile west of Spur, Dickens County. Drainage area. $--0.0131 \mathrm{sq} \mathrm{mi}$.

Gage.--Recording.

Remarks.--Records furnished by U.S. Department of Agriculture, Agricultural Research Service. Only annual (calendar year) peaks are shown.

Peak stages and discharges

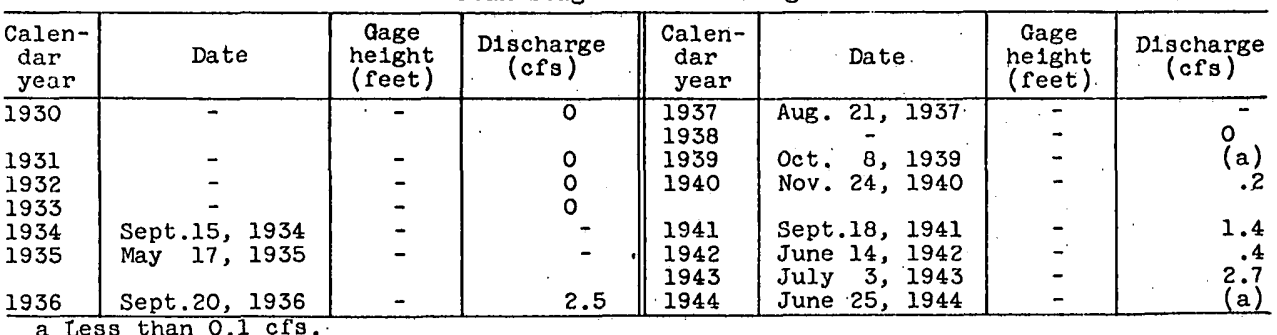

8-809.47. Unnamed tributary (watershed 14) of Duck Creek near'Spur, Tex.(25)

Location.--Lat $33^{\circ} 28^{\prime}$, Iong $100^{\circ} 53^{\prime}, 1$ mile west of Spur, Dickens County.

Drainage area. $-0.0133 \mathrm{sq} \mathrm{m} !$.

Gage.--Recording.

Remarks.--Records furnished by U.S. Department of Agriculture; Agricultural Research Service. Only annual (calendar year) peaks are shown.

\begin{tabular}{|c|c|c|c|c|c|c|c|}
\hline $\begin{array}{l}\text { Calen- } \\
\text { dar } \\
\text { year }\end{array}$ & Date & $\begin{array}{c}\text { Gage } \\
\text { nelght } \\
\text { (feet) }\end{array}$ & $\begin{array}{c}\text { Discharge } \\
(\mathrm{cfs})\end{array}$ & $\begin{array}{l}\text { Calen- } \\
\text { dar } \\
\text { year }\end{array}$ & Date & $\begin{array}{c}\text { Gage } \\
\text { he1ght } \\
\text { (feet) }\end{array}$ & $\begin{array}{c}\text { D1scharge } \\
\text { (cfs) }\end{array}$ \\
\hline 1930 & Dec. 4,1930 & - & 0.4 & $\begin{array}{l}1937 \\
1938\end{array}$ & $\begin{array}{l}\text { Aug. } 24,1937 \\
\text { June } 4 ;, 1938\end{array}$ & $\overline{-}$ & $\begin{array}{l}2.8 \\
2.0\end{array}$ \\
\hline $\begin{array}{l}1931 \\
1932 \\
1933\end{array}$ & $\begin{array}{lrl}\text { May } & 25, & 1931 \\
\text { June } & 20, & 1932 \\
\text { July } & 6, & 1933\end{array}$ & $\overline{-}$ & $\begin{array}{l}1.1 \\
6.0 \\
1.3\end{array}$ & $\begin{array}{l}1939 \\
1940\end{array}$ & $\begin{array}{l}\text { Oct. } 8,1939 \\
\text { Nov. } 24,1940\end{array}$ & - & $\begin{array}{l}.9 \\
1.6\end{array}$ \\
\hline 1934 & Sept.15, 1934 & - & - & 1941 & Apr. 29, 1941 & - & 5.2 \\
\hline 1935 & May 17,1935 & - & 2.8 & $\begin{array}{l}1942 \\
1943\end{array}$ & $\begin{array}{lr}\text { June } & 14, \\
\text { July } & 1942 \\
1943\end{array}$ & $\overline{-}$ & $\begin{array}{l}2.6 \\
5.8\end{array}$ \\
\hline 1936 & Sept.20, 1936 & - & 10 & & & & \\
\hline
\end{tabular}

\section{8-809.49. Unnamed tributary (watershed 15) of Duck Creek near Spur, Tex.(25)}

Location.--Lat $33^{\circ} 28^{\prime}$, Iong $100^{\circ} 53^{\prime}$, 1 mile west of Spur, Dickens County.

Drainage area. $--0.0133 \mathrm{sq} \mathrm{ml}$.

Gage.--Recording.

Remarks.--Records furnished by U.S. Department of Agriculture, Agricultural Research Service. Only annual (calendar year) peaks are shown.

\begin{tabular}{|c|c|c|c|c|c|c|c|}
\hline $\begin{array}{l}\text { Calen- } \\
\text { dar } \\
\text { year }\end{array}$ & Date & $\begin{array}{c}\text { Gage } \\
\text { height } \\
\text { (feet) }\end{array}$ & $\begin{array}{c}\text { Discharge } \\
\text { (cfs) }\end{array}$ & $\begin{array}{l}\text { Calen- } \\
\text { dar } \\
\text { year } \\
\end{array}$ & Date & $\begin{array}{c}\text { Gage } \\
\text { helght } \\
\text { (feet) }\end{array}$ & $\begin{array}{l}\text { Discharge } \\
\text { (cfs) }\end{array}$ \\
\hline 1930 & oct. 23,1930 & - & 0.1 & $\begin{array}{l}1937 \\
1938\end{array}$ & Aug. 24,1937 & $\overline{-}$ & 0.8 \\
\hline $\begin{array}{l}1931 \\
1932 \\
1933\end{array}$ & June $2 \overline{0}, 1932$ & - & $\begin{array}{l}0 \\
0\end{array}$ & $\begin{array}{l}1939 \\
1940\end{array}$ & $\begin{array}{lr}\text { Oct. } 8,1939 \\
\text { Nov. } 24,1940\end{array}$ & - & (a) \\
\hline $\begin{array}{l}1934 \\
1935\end{array}$ & $\begin{array}{l}\text { Sept.15, } 1934 \\
\text { June } 7,1935\end{array}$ & - & - & $\begin{array}{l}1941 \\
1942\end{array}$ & $\begin{array}{l}\text { Sept.18, } 1941 \\
\text { June } 14,1942\end{array}$ & - & 3.0 \\
\hline 1936 & Sept.20, 1936 & - & 5.2 & $\begin{array}{l}1943 \\
1944\end{array}$ & $\begin{array}{l}\text { July } 3,1943 \\
\text { June } 25,1944\end{array}$ & - & (a) \\
\hline
\end{tabular}


Location.--Lat $33^{\circ} 24^{\prime} 05^{\prime \prime}$, long $100^{\circ} 24^{\prime} 30^{\prime \prime}$, on left bank 0.1 mile downstream from Haystack Creek, 2.4 miles downstream from Salt Flat Creek, 9.3 miles upstream from Salt Fork Brazos River, and 21 miles northwest of Aspermont, Stonewall County.

Drainage area. $---64.3 \mathrm{sq} \mathrm{mi}$.

Gage.--Recording.

Stage-discharge relation.--Defined by current-meter measurements below 250 cfs and extended above by slope-area measurements at $4.96 \mathrm{ft}(6,910 \mathrm{cfs})$ and $6.42 \mathrm{ft}(11,400 \mathrm{cfs})$.

Bankfull stage.--3.0 ft.

Historical data.--Flood of 1941 reached a stage of about $9 \mathrm{ft}$, from information by local residents.

Remarks.--Base for partial-duration series, 1,000 cfs. Prior to 1959 published as Dove. Creek near Aspermont, Tex. Rain gage at site.

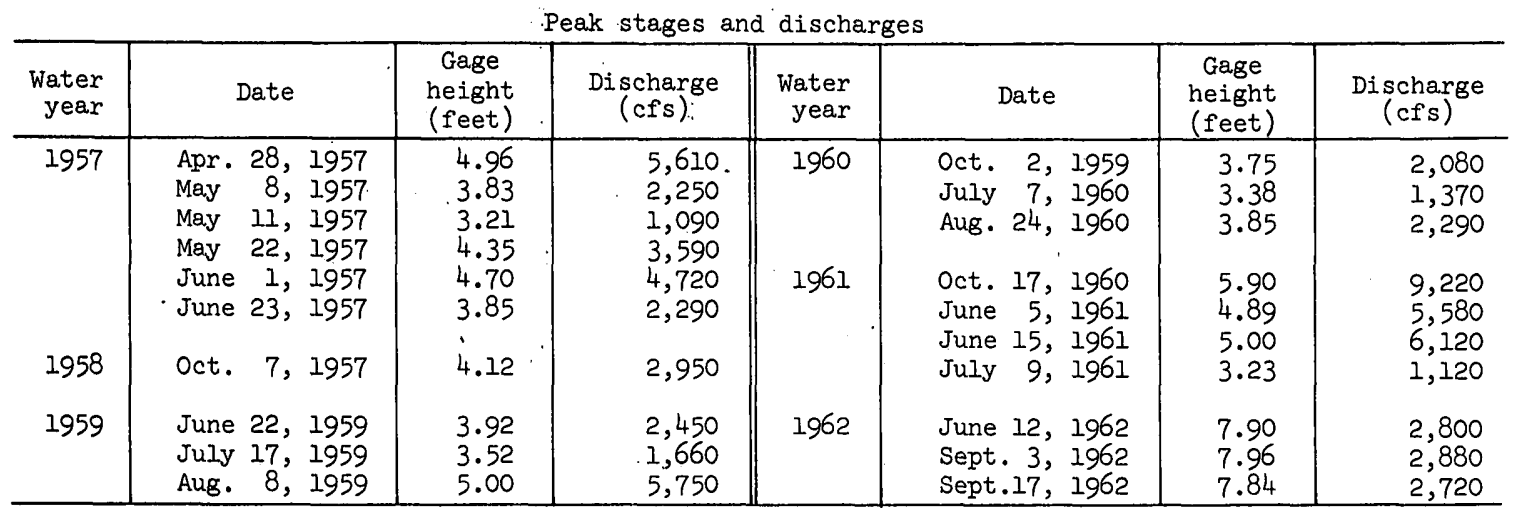

8-861.5 North Fork Hubbard Creek near Albany, Tex. (8)

Location.--Lat $32^{\circ} 42^{\prime} 20^{\prime \prime}$, Long $99^{\circ} 16^{\prime} 20^{\prime \prime}$, on downstream side of bridge on U. S. Highway 380 , 1.6 miles upstream from Salt Prong Hubbard Creek, and 1.7 miles southeast of Albany, Shackelford County.

Drainage area.--38.4 sq mi.

Gage.--Recording. Datum of gage is 1,340.54 ft above mean sea level, datum of 1929.

Stage-discharge relation.--Defined by current-meter measurements below $100 \mathrm{cfs}$.

Bankfull stage. $--16 \mathrm{ft}$.

Historical data.--Highest stage since 1940, about $21 \mathrm{ft}$ on June 10, 1940, and July 18, 1953, from information by local residents.

Remarks.--Base for partial-duration series, $100 \mathrm{cfs}$.

Peak stages and discharges

\begin{tabular}{c|c|c|c||c|c|c|c}
\hline $\begin{array}{c}\text { Water } \\
\text { year }\end{array}$ & Date & $\begin{array}{c}\text { Gage } \\
\text { height } \\
\text { (feet) }\end{array}$ & $\begin{array}{c}\text { Discharge } \\
\text { (cfs) }\end{array}$ & $\begin{array}{c}\text { Water } \\
\text { year. }\end{array}$ & Date & $\begin{array}{c}\text { Gage } \\
\text { height } \\
\text { (feet) }\end{array}$ & $\begin{array}{c}\text { Discharge } \\
\text { (cfs) }\end{array}$ \\
\hline 1963 & June 11,1963 & 3.72 & 134 & & & & \\
\hline
\end{tabular}


8-881. Salt Creek at Olney, Tex. (3)

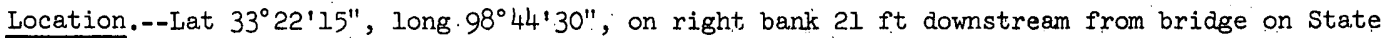
Highway 199 and 0.5 mile east of Olney, Young County..

Drainage area. $--9.6 \mathrm{sq} \mathrm{mi}$.

Gage.--Recording. Datum of gage is 1,164.03 ft above mean sea level, datum of 1929.

Stage-discharge relation.--Defined by current-meter measurements below 680 cfs and extended above by slope-conveyance studies.

Bankfull stage. -- 7 ft.

Historical data.--Maximum stage since at least 1908, 16.7. ft in June 1915, from information by local residents.

Remarks.--Base for partial-duration series, $200 \mathrm{cfs}$. Rain gage at site.

\begin{tabular}{|c|c|c|c|c|c|c|c|}
\hline $\begin{array}{r}\text { Water } \\
\text { year }\end{array}$ & Da.te & $\begin{array}{c}\text { Gage } \\
\text { height } \\
\text { (feet) }\end{array}$ & $\begin{array}{l}\text { Discharge } \\
\text { (cfs) }\end{array}$ & $\begin{array}{r}\text { Water } \\
\text { yeer }\end{array}$ & Date & $\begin{array}{c}\text { Gage } \\
\text { height } \\
\text { (feet) }\end{array}$ & $\begin{array}{l}\text { Discharge } \\
\text { (cfs) }\end{array}$ \\
\hline 1958 & $\begin{array}{ll}\text { May } 1, & 1958 \\
\text { July 6, } & 1958 \\
\text { Sept.16, } & 1958\end{array}$ & $\begin{array}{l}7.44 \\
7.84 \\
8.18\end{array}$ & $\begin{array}{l}262 \\
309 \\
345\end{array}$ & $\begin{array}{l}1961 . \\
1962 .\end{array}$ & $\begin{array}{l}\text { Sept.12, } 1961 \\
\text { Nov. 22, } 1961 \\
\text { June 9, } 1962\end{array}$ & $\begin{array}{l}5.95 \\
9.66 \\
9.04\end{array}$ & $\begin{array}{l}162 \\
485 \\
299\end{array}$ \\
\hline 1959 & June 22, 1959 & $7 \cdot 30$ & 264 & . & Sept. 6, 1962 & 9.28 & 360 \\
\hline 1960 & $\begin{array}{lr}\text { Oct. } 3, & 1959 \\
\text { July } 13, & 1960 \\
\end{array}$ & $\begin{array}{r}10.16 \\
8.43 \\
\end{array}$ & $\begin{array}{r}1,040 \\
392 \\
\end{array}$ & 1963 & $\begin{array}{l}\text { Nov. 26, } 1962 \\
\text { June } 1,1963\end{array}$ & $\begin{array}{l}9.32^{\circ} \\
8.66 \\
\end{array}$ & $\begin{array}{l}360 \\
253 \\
\end{array}$ \\
\hline
\end{tabular}

8-882. Salt Creek near Newcastle, Tex. (3)

Location.--Lat $33^{\circ} 13^{\prime} 00^{\prime \prime}$, long $98^{\circ} 38^{\prime} 55^{\prime \prime}$, on left bank 75 ft downstream from county bridge, i.0 mile upstream from Oak Creek, and 5.0 miles east of Newcastle, Young County.

Drainage area. $--57.9 \mathrm{sq} \mathrm{mi}$.

Gage.--Recording.

Stage-discharge relation.--Defined by current-meter measurements below $10 \mathrm{cfs}$ and extended above on the basis of slope-conveyance studies.

Bankfull stage.--10 ft.

Historical da.ta.--Maximum flood known occurred in 1900 (stage and discharge unknown). Maximum stage since 1900 in 1941 and September 1955, gage height $20.6 \mathrm{ft}$, from information by local residents.

Remarks.--Base for partial-duration series, $400 \mathrm{cfs}$.

Peak stages and discharges

\begin{tabular}{c|c|c|c||c|c|c|c}
\hline $\begin{array}{c}\text { Water } \\
\text { year }\end{array}$ & Da.te & $\begin{array}{c}\text { Gage } \\
\text { height } \\
\text { (feet) }\end{array}$ & $\begin{array}{c}\text { Discharge } \\
\text { (cfs) }\end{array}$ & $\begin{array}{c}\text { Water } \\
\text { year }\end{array}$ & Date & $\begin{array}{c}\text { Ga.ge } \\
\text { height } \\
\text { (feet) }\end{array}$ & $\begin{array}{c}\text { Discharge } \\
\text { (cfs) }\end{array}$ \\
\hline 1959 & July 19,1959 & 6.33 & 386 & 1960 & Feb. 3, 1960 & 8.08 & 690 \\
1960 & Oct. 4, 1959 & 16.92 & 3,840 & & July 14, 1960 & 7.40 & 572 \\
\hline
\end{tabular}


Location.--Lat $33^{\circ} 12^{\prime} 40^{\prime \prime}$, long $98^{\circ} 37^{\prime} 05^{\prime \prime}$, on downstream side of bridge on Farm Road $1769,2.5 \mathrm{miles}$ upstream from mouth, and 7.0 miles northwest of Graham, Young County.

Drainage area. $--19.7 \mathrm{sq} \mathrm{mi}$.

Gage.--Recording.

Stage-discharge relation.--Defined by current-meter measurements below $330 \mathrm{cfs}$, and extended above by logarithmic plotting.

Bankfull stage. $--8 \mathrm{ft}$.

Historical data.--Maximum stage since at least 1900, $15.2 \mathrm{ft}$ in September 1955, from information by local residents.

Remarks.--Base for partial-duration series, 200 cfs.

Peak stages and discharges

\begin{tabular}{|c|c|c|c|c|c|c|c|}
\hline $\begin{array}{l}\text { Wa.ter } \\
\text { year }\end{array}$ & Date & $\begin{array}{c}\text { Gage } \\
\text { height } \\
\text { (feet) }\end{array}$ & $\begin{array}{c}\text { Discharge } \\
\text { (cfs) }\end{array}$ & $\begin{array}{l}\text { Water } \\
\text { year }\end{array}$ & Date & $\begin{array}{c}\text { Gage } \\
\text { height } \\
\text { (feet) }\end{array}$ & $\begin{array}{l}\text { Discharge } \\
\text { (cfs) }\end{array}$ \\
\hline $\begin{array}{l}1959 \\
1960\end{array}$ & $\begin{array}{l}\text { June 23, } 1959 \\
\text { Oct. } 3,1959 \\
\text { Feb. } 3,1960 \\
\text { July } 7,1960\end{array}$ & $\begin{array}{l}4.08 \\
9.20 \\
4.25 \\
5.68\end{array}$ & $\begin{array}{l}207 \\
649 \\
219 \\
303\end{array}$ & 1962 & $\begin{array}{ll}\text { Mar. } 20, & 1962 \\
\text { June 10, } 1962 \\
\text { July 27, } 1962 \\
\text { Sept. 7, } 1962\end{array}$ & $\begin{array}{r}4.86 \\
10.50 \\
9.60 \\
7.08\end{array}$ & $\begin{array}{l}220 \\
563 \\
513 \\
368\end{array}$ \\
\hline 1961 & $\begin{array}{l}\text { Oct. } 18,1960 \\
\text { Jan. } 8,1961 \\
\text { Mar. } 18,1961\end{array}$ & $\begin{array}{l}8.42 \\
4.78 \\
4.18 \\
\end{array}$ & $\begin{array}{l}555 \\
261 \\
219 \\
\end{array}$ & 1963 & $\begin{array}{l}\text { Apr. 27, } 1963 \\
\text { June 1, } 1963\end{array}$ & $\begin{array}{l}5.10 \\
4.97\end{array}$ & $\begin{array}{l}245 \\
239\end{array}$ \\
\hline
\end{tabular}

8-937. North Bosque River at Stephenville, Tex. (2)

Location.--Lat $32^{\circ} 12^{\prime} 55^{\prime \prime}$, long $98^{\circ} 11^{\prime} 50^{\prime \prime}$, in center of stream on downstream side of bridge on U. S. Highway 67 at Stephenville, Erath County.

Drainage area.--93.2 sq $\mathrm{mi}$.

Gage.--Recording. Da.tum of gage is $1,223.60 \mathrm{ft}$ above mean sea level, datum of 1929, supplementary adjustment of 1942.

Stage-discharge relation.--Defined by current-meter measurements below 4,200 cfs, and extended above on the basis of indirect measurements of 40,000 and 49,000 cfs.

Bankfull stage.--15 ft.

Historical data.--Maximum stage since at least 1854, $23.5 \mathrm{ft}$ May 19, 1955, from floodmarks

(discharge, 49,000 cfs), from slope-area measurement of peak flow.

Remarks.--Base for partial-duration series, 1,000 cfs. Rain gage at site.

Peak stages and discharges

\begin{tabular}{|c|c|c|c|c|c|c|c|}
\hline $\begin{array}{r}\text { Water } \\
\text { year }\end{array}$ & Date & $\begin{array}{c}\text { Gage } \\
\text { height } \\
\text { (feet) }\end{array}$ & $\begin{array}{c}\text { Discharge } \\
\text { (cfs) }\end{array}$ & $\begin{array}{r}\text { Water } \\
\text { year }\end{array}$ & Date & $\begin{array}{c}\text { Gage } \\
\text { height } \\
\text { (feet) }\end{array}$ & $\begin{array}{c}\text { Discharge } \\
\text { (cfs) }\end{array}$ \\
\hline 1959 & $\begin{array}{lrl}\text { Apr. } & 30, & 1959 \\
\text { May } & 3,1959 \\
\text { July } & 6, & 1959\end{array}$ & $\begin{array}{r}6.55 \\
9.90 \\
18.26\end{array}$ & $\begin{array}{r}691 \\
1,460 \\
6,850\end{array}$ & 1961 & $\begin{array}{l}\text { June } 3,1961 \\
\text { June } 15,1961 \\
\text { June } 17,1961\end{array}$ & $\begin{array}{r}12.90 \\
9.52 \\
10.35\end{array}$ & $\begin{array}{l}2,770 \\
1,490 \\
1,740\end{array}$ \\
\hline 1960 & $\begin{array}{ll}\text { Oct. } & 4,1959 \\
\text { Nov. } & 4,1959 \\
\text { Jan. } & 5,1960\end{array}$ & $\begin{array}{r}19.90 \\
7.26 \\
8.91\end{array}$ & $\begin{array}{r}12,100 \\
1,140 \\
1,670\end{array}$ & 1962 & $\begin{array}{l}\text { Oct. 9, } 1961 \\
\text { Sept. 7, } 1962\end{array}$ & $\begin{array}{l}11.43 \\
10.75\end{array}$ & $\begin{array}{l}2,030 \\
1,850\end{array}$ \\
\hline 1961 & $\begin{array}{l}\text { Apr. } 27,1960 \\
\text { Jan. } 7,1961\end{array}$ & $\begin{array}{c}9.60 \\
10.03\end{array}$ & $\begin{array}{l}1,830 \\
1,630\end{array}$ & 1963 & $\begin{array}{l}\text { Oct. } 8,1962 \\
\text { Apr. } 28,1963 \\
\text { May } 28,1963 \\
\text { June } 16,1963\end{array}$ & $\begin{array}{l}15.37 \\
12.70 \\
15.10 \\
11.63 \\
\end{array}$ & $\begin{array}{l}3,250 \\
2,210 \\
3,100 \\
1,910\end{array}$ \\
\hline
\end{tabular}


8-940. Green Creek subwatershed No. 1 near Dublin, Tex. (2)

Location.--Lat $32^{\circ} 10^{\prime} 00^{\prime \prime}$, long $98^{\circ} 20^{\prime} 30^{\prime \prime}$, near center of dam on main headwater channel of Green Creek, three-quarter's of a mlle downstream from county road, 1.0 mile east of Farm Road 219, and 4.0 miles north of Dublin; Erath County.

Drainage area. $--3.18 \mathrm{sq} \mathrm{ml}$.

Gage.--Recording. Datum of gage is $1,408.00 \mathrm{ft}$ above mean sea level, datum of I929 (levels by U.S. Soll Conservation Service).

Remarks.--Peaks are based on maximum inflow (average for 15-minute interval), computed from outflow and change in reservoir contents, adjusted for rainfall on the reservolr surface during time of peak inflow. No adjustment made for reservoir losses. Base for partiai-duration series, $125 \mathrm{cfs}$.

Peak stages and discharges

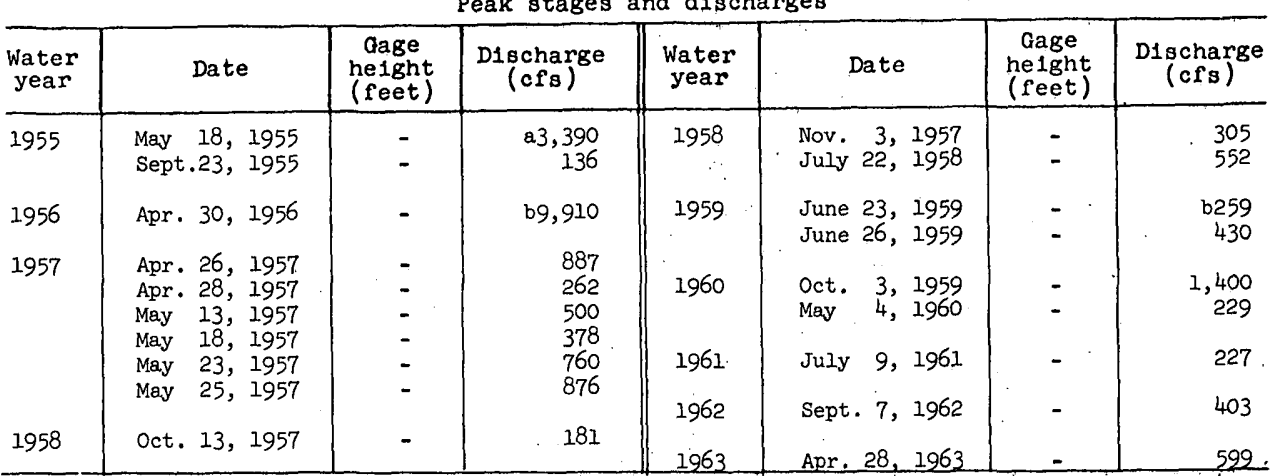

a First appreciable inflow since dam was completed in April 1955

$b$ Not adjusted for rainfall on water surface.

Note.--Only annual peaks after 1960.

8-945. Green Creek near Alexander, Tex.(2)

Location.--Lat $32^{\circ} 04^{\prime} 20^{\prime \prime}$, long $98^{\circ} 14^{\prime} 00^{\prime \prime}$, at downstream side of bridge on state Highway $6,0.2 \mathrm{mlle}$ upstream from Missourl, Kansas, Texas Ra1lroad Co. bridge, I.0 mile upstream from Cottonwood Creek, and 1.7 miles northwest of Alexander, Erath County.

Drainage area. $--45.5 \mathrm{sq} \mathrm{ml}$.

Gage.--Crest-stage gage prior to May 27, 1958; recording and crest-stage gage thereafter. Datum of gage is $1,172 \mathrm{ft}$ above mean sea level, datum of 1929 .

Stage-discharge relation.--Defined by current-meter measurements below $920 \mathrm{cfs}$ and by contracted-opening measurements at 23,900 and $55,800 \mathrm{cfs}$.

Bankfull stage. $--20 \mathrm{ft}$.

Historical data. - Flood of May 23, 1952, reached highest stage since at least 1910, from information by locai resident. Also a local resident stated there was a very high flood in 1918.

Remarks.--Between 1954 and September 1956, elght floodwater-retarding structures were bullt. These structures have a total floodwater-detention capacity of 7,840 acre-ft below flood splllway crests and partly control the flow from $22.8 \mathrm{sq} \mathrm{ml}$ above the station. Only annual peaks are shown.

\begin{tabular}{|c|c|c|c|c|c|c|c|c|}
\hline $\begin{array}{l}\text { Water } \\
\text { year }\end{array}$ & & Date & $\begin{array}{c}\text { Qage } \\
\text { helght } \\
\text { (feet) }\end{array}$ & $\begin{array}{c}\text { D1scharge } \\
\text { (cfs) }\end{array}$ & $\begin{array}{l}\text { Water } \\
\text { year }\end{array}$ & Date & $\begin{array}{c}\text { Gage } \\
\text { helght } \\
\text { (feet) }\end{array}$ & $\begin{array}{c}\text { Discharge } \\
(\mathrm{cfs})\end{array}$ \\
\hline 1952 & May & 23,1952 & 28.0 & 55,800 & 1958 & October 1957 & 8.49 & 1,170 \\
\hline 1955 & May & 19,1955 & & & $\begin{array}{l}1959 \\
1960\end{array}$ & $\begin{array}{l}\text { June 26, } 1959 \\
\text { Oct. 4, } 1959\end{array}$ & $\begin{array}{r}5.70 \\
12.14\end{array}$ & $\begin{array}{r}274 \\
3,190\end{array}$ \\
\hline $\begin{array}{l}1956 \\
1957\end{array}$ & $\begin{array}{l}\text { Apr. } \\
\text { Apr. }\end{array}$ & $\begin{array}{l}30,1956 \\
26,1957\end{array}$ & $\begin{array}{l}23.95 \\
14.76\end{array}$ & $\begin{array}{r}23,900 \\
5,400\end{array}$ & $\begin{array}{l}1961 \\
1962 \\
1963 \\
\end{array}$ & $\begin{array}{l}\text { Jan. } 7,1961 \\
\text { Oct. } 9,1961 \\
\text { June } 16,1963\end{array}$ & $\begin{array}{r}6.80 \\
11.70 \\
9.15 \\
\end{array}$ & $\begin{array}{r}580 \\
2,880 \\
1,460 \\
\end{array}$ \\
\hline
\end{tabular}

Note.--Peak discharge shown for Apr. 30, 1956, includes undetermined amount of flow over emergency spillways of floodwater-retarding structures. Some of the other peak discharges given since 1955 include up to about $160 \mathrm{cfs}$ of combined service spiliway discharge. 
Loca.tion.--Lat $31^{\circ} 33^{\prime} 20^{\prime \prime}$, long $97^{\circ} 21^{\prime} 22^{\prime \prime}$, on downstream side of bridge on Farm Road $185,5.6$ miles east of Crawford, McLennan County.

Drainage area. $--78.2 \mathrm{sq} \mathrm{mi}$.

Gage.--Recording. Datum of gage is $560.54 \mathrm{ft}$ above mean sea level, datum of 1929 . Prior to Oct. 27, 1959, wire-weight gage at same site and datum.

Stage-discharge relation.--Defined by current-meter measurements.

Bankfull stage.--13 ft.

Historical data.--Maximum stage since 1900, $17.5 \mathrm{ft}$ Sept. 26, 1936, from information by local residents.

Remarks.--Base for partial-duration series, 1,600 cfs. Rain gage at site.

\begin{tabular}{|c|c|c|c|c|c|c|c|}
\hline $\begin{array}{r}\text { Water } \\
\text { year }\end{array}$ & Date & $\begin{array}{c}\text { Gage } \\
\text { height } \\
\text { (feet) }\end{array}$ & $\begin{array}{c}\text { Discharge } \\
\quad(\mathrm{cfs})\end{array}$ & $\begin{array}{r}\text { Water } \\
\text { year }\end{array}$ & Date & $\begin{array}{c}\text { Gage } \\
\text { height } \\
\text { (feet) }\end{array}$ & $\begin{array}{c}\text { Discharge } \\
\text { (cfs) }\end{array}$ \\
\hline 1960 & $\begin{array}{l}\text { Oct. } 4,1959 \\
\text { Oct. } 13,1959 \\
\text { Jan. } 5,1960 \\
\text { July } 7,1960\end{array}$ & $\begin{array}{r}14.31 \\
6.30 \\
5.58 \\
9.60\end{array}$ & $\begin{array}{r}15,400 \\
2,590 \\
1,820 \\
7,310\end{array}$ & 1961 & $\begin{array}{l}\text { Feb. } 16,1961 \\
\text { June } 15,1961 \\
\text { June 18, } 1961 \\
\text { July 16, } 1961\end{array}$ & $\begin{array}{l}8.32 \\
7.20 \\
7.95 \\
6.05\end{array}$ & $\begin{array}{l}5,300 \\
3,730 \\
4,850 \\
2,300\end{array}$ \\
\hline \multirow[t]{2}{*}{1961} & $\begin{array}{l}\text { Oct. } 28,1960 \\
\text { Dec. } 8,1960 \\
\text { Dec. 10, } 1960 \\
\text { Jan. } 8,1961 \\
\text { Jan. } 12,1961 \\
\text { Feb. } 5,1961\end{array}$ & $\begin{array}{l}5.67 \\
6.43 \\
5.40 \\
7.87 \\
6.25 \\
7.46\end{array}$ & $\begin{array}{l}1,870 \\
2,770 \\
1,620 \\
4,710 \\
2,530 \\
4,150\end{array}$ & 1962 & $\begin{array}{l}\text { Oct. 10, } 1961 \\
\text { Nov. 22, } 1961 \\
\text { Dec. 9, } 1961 \\
\text { Apr. 30, } 1962 \\
\text { Sept. 8, } 1962\end{array}$ & $\begin{array}{r}11.50 \\
9.50 \\
5.54 \\
6.53 \\
7.98\end{array}$ & $\begin{array}{r}10,500 \\
7,150 \\
1,770 \\
2,890 \\
4,850\end{array}$ \\
\hline & & & & 1963 & 6,1963 & 6.33 & 2,650 \\
\hline
\end{tabular}

8-967. Sandy Creek watershed SW-16 near Riesel, Tex.(9)

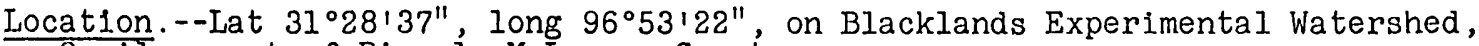
2 miles east of Riesei, McLennan County.

Drainage area. $--0.0050 \mathrm{sq} \mathrm{m}$.

Gage.--Recording. Datum of gage is $536.9 \mathrm{ft}$ above mean sea level.

Stage-discharge relation.--Defined by theoretical rating for 2-foot Parshall flume.

Remarks.--Records furnished by U.S. Department of Agriculture, Agricultural Research Service, Blacklands Experimental Watershed at Riesel, Tex. Detailed information is avallable from the Riesel office regarding bas in shape and slope, type of soils, erosion condition, watershed conditions (kind of soll cover that bear a relationship to hydrology, and rainfali. Only annual (calendar year) peaks are shown.

Peak stages and discharges

\begin{tabular}{|c|c|c|c|c|c|c|c|}
\hline $\begin{array}{l}\text { Calen- } \\
\text { dar } \\
\text { year }\end{array}$ & Date & $\begin{array}{c}\text { Gage } \\
\text { nelght } \\
\text { (feet) }\end{array}$ & $\begin{array}{c}\text { Discharge } \\
\text { (cfs) }\end{array}$ & $\begin{array}{l}\text { Calen- } \\
\text { dar } \\
\text { year }\end{array}$ & Date & $\begin{array}{c}\text { Gage } \\
\text { nelght } \\
\text { (feet) }\end{array}$ & $\begin{array}{c}\text { Discharge } \\
\text { (cfs) }\end{array}$ \\
\hline $\begin{array}{l}1938 \\
1939 \\
1940 \\
\end{array}$ & $\begin{array}{l}\text { Apr. } 15,1938 \\
\text { May } 17,1939 \\
\text { Oct. } 31,1940\end{array}$ & $\overline{-}$ & $\begin{array}{l}1.3 \\
6.1 \\
19\end{array}$ & $\begin{array}{l}1941 \\
1942 \\
1943\end{array}$ & $\begin{array}{l}\text { June } 10,1941 \\
\text { Sept. } 8,1942 \\
\text { June } 5,1943 \\
\end{array}$ & - & $\begin{array}{l}14 \\
12 \\
12 \\
\end{array}$ \\
\hline
\end{tabular}


8-968. Cow Bayou subwatershed No. 4 near Bruceville, Tex. (9)

Location.--Lat $31^{\circ} 20^{\prime}$, long $97^{\circ} 16^{\prime}$, near center of dam on Foster Branch, 1.0 mile upstream from South Fork Cow Bayou, 2.1 miles west of Bruceville, McLennan County, and 2.3 miles northwest of Eddy.

Drainage area. $--5.25 \mathrm{sq} \mathrm{mi}$.

Gage.--Recording. Datum of gage is $574.46 \mathrm{ft}$ above mean sea level, datum of 1929 (levels by U. S. Soil Conservation Service).

Remarks.--Peak discharge based on maximum inflow (average for 15-minute interval), computed from outflow and change in reservoir contents, adjusted for rainfall on the reservoir surface during time of peak inflow. Only annual peaks are shown.

\begin{tabular}{c|c|c|c||c|c|c|c}
\hline $\begin{array}{c}\text { Water } \\
\text { year }\end{array}$ & Date & $\begin{array}{c}\text { Gage } \\
\text { height } \\
\text { (feet) }\end{array}$ & $\begin{array}{c}\text { Discharge } \\
\text { (cfs) }\end{array}$ & $\begin{array}{c}\text { Water } \\
\text { year }\end{array}$ & Date & $\begin{array}{c}\text { Gage } \\
\text { height } \\
\text { (feet) }\end{array}$ & $\begin{array}{c}\text { Discharge } \\
\text { (cfs) }\end{array}$ \\
\hline 1957 & May 11, 1957 & - & 6,900 & 1961 & June 8, 1961 & - & \\
1958 & Oct. 14, 1957 & - & $\begin{array}{c}1,510 \\
1,690\end{array}$ & 1962 & June 30, 1962 & - & 628 \\
1959 & June 23, 1959 & - & Oct. 26, 1962 & - & 293 \\
1960 & 0ct. 4, 1959 & - & 1,400 & & 19 \\
\hline
\end{tabular}

\section{8-970. Cow Bayou at Mooreville, Tex.(9)}

Location.--Lat $31^{\circ} 18^{\prime} 45^{\prime \prime}$, long $97^{\circ} 08^{\prime} 16^{\prime \prime}$, on right bank at downstream side of county bridge, $5.00 \mathrm{ft}$ downstream from confluence of North Cow Bayou and South Cow Bayou, 0.8 mile north of Mooreville, Falls County, and 5.0 miles northwest of Chilton.

Drainage area. $--79.6 \mathrm{sq} \mathrm{m} 1$.

Gage.--Nonrecording prior to June 10, 1958; recording thereafter. Datum of gage is $399.58 \mathrm{ft}$ above mean sea level, datum of 1929 (levels by U.S. Soil Conservation Service).

Stage-discharge relation.--Defined by current-meter measurements below 4,500 cf's and extended above to 7,960 cfs on basis of logarithmic plotting.

Bankfull stage. $--14 \mathrm{ft}$.

Historical data.--Maximum stage since at least 1900, that of May 1, 1944.

Remarks. - :... itum $28.0 \mathrm{sq}$ mi ab. station is partly controlled by nine floviater-detention structures with a total combined capacity, of 9,770 acre-ft below spillway crest. First structure completed in April 1955; last structure completed in June 1958. Only annual peaks are shown.

Peak stages and discharges

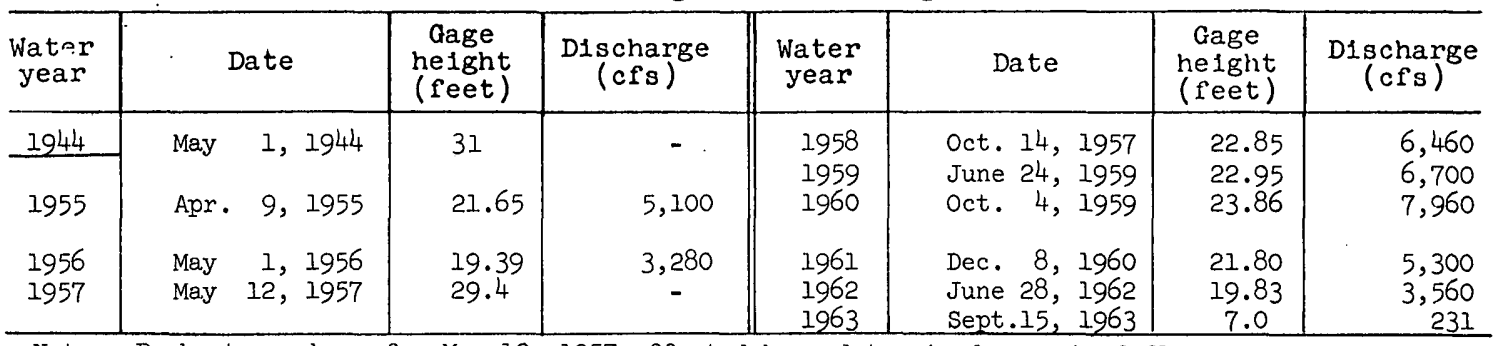

Note.--Peak stage shown for May 12, 1957 affected by undetermined amount of flow over emergency spillways of floodwater-retarding structures. Peak discharges shown since 1955 include up to about $250 \mathrm{cfs}$ of combined service spillway discharge. 
Location.--Lat $31^{\circ} 16^{\prime}$, long $97^{\circ} 04^{\prime}, 75 \mathrm{ft}$ downstream from San Antonio and Aransas Pass Railway bridge and $0.8 \mathrm{mile}$ south of Chilton, Falls County.

Drainage area. $--81.8 \mathrm{sq} \mathrm{mi}$.

Gage.--Recording. Datum of gage is $372.60 \mathrm{ft}$ above mean sea level, datum of 1929.

Historical data.--Flood of September 1921 reached a stage of about $20.5 \mathrm{ft}$, from information by local residents.

Remarks.--Only maximum annual discharge shown.

Peak stages and discharges

\begin{tabular}{c|c|c|c||c|c|c|c}
\hline $\begin{array}{c}\text { Water } \\
\text { year }\end{array}$ & Date & $\begin{array}{c}\text { Gage } \\
\text { height } \\
\text { (feet) }\end{array}$ & $\begin{array}{c}\text { Discharge } \\
\text { (cfs) }\end{array}$ & $\begin{array}{c}\text { Water } \\
\text { year }\end{array}$ & Date & $\begin{array}{c}\text { Gage } \\
\text { height } \\
\text { (feet) }\end{array}$ & $\begin{array}{c}\text { Discharge } \\
\text { (cfs) }\end{array}$ \\
\hline 1934 & Apr. 6, 1934 & $\begin{array}{l}13.2 \\
16.08\end{array}$ & $\begin{array}{r}4,000 \\
14,500\end{array}$ & 1936 & Dec. 6, 1935 & 16.46 & 16,000 \\
\hline 1935 & May 18, 1935 & 16.08 & \\
\hline
\end{tabular}

8-982. Brushy Creek watershed A near Riesel, Tex. (9)

Location.--Lat $31^{\circ} 32^{\prime} 10^{\prime \prime}$, long $96^{\circ} 53^{\prime} 33^{\prime \prime}, 4.8$ miles northeast of Riesel, McLennan County.

Drainage area. $--0.0656 \mathrm{sq} \mathrm{m1}$.

Gage.--Recording. Datum of gage is $573.3 \mathrm{ft}$ above mean sea level.

Stage-discharge relation.--Defined by theoretical rating for a 10-foot Parshall flume and checked by current meter.

Remarks.--Records furnished by U.S. Department of Agriculture, Agricultural Research Service, Blacklands Experimental Watershed at Riesel, Tex. Detailed information is avallable from the Riesel office regarding basin shape and slope, type of soils, erosion conditions, watershed conditions (kind of soil cover that bear a relationship to hydrology, and rainfall. The drainage area is artificially controlled, therefore no gage heights are shown. Only annual (calendar year) peaks are shown.

Peak stages and discharges

\begin{tabular}{|c|c|c|c|c|c|c|c|}
\hline $\begin{array}{l}\text { Calen- } \\
\text { dar } \\
\text { year }\end{array}$ & Date & $\begin{array}{c}\text { Gage } \\
\text { nelght } \\
\text { (feet) }\end{array}$ & $\begin{array}{c}\text { Discharge } \\
\text { (cfs) }\end{array}$ & $\begin{array}{l}\text { Calen- } \\
\text { dar } \\
\text { year }\end{array}$ & Date & $\begin{array}{c}\text { Gage } \\
\text { ne1ght } \\
\text { (feet) }\end{array}$ & $\begin{array}{c}\text { D1scharge } \\
\text { (cfs) }\end{array}$ \\
\hline $\begin{array}{l}1939 \\
1940\end{array}$ & $\begin{array}{ll}\text { June } 19, & 1939 \\
\text { Nov. } 22, & 1940\end{array}$ & - & $\begin{array}{l}15 \\
24\end{array}$ & $\begin{array}{l}1942 \\
1943\end{array}$ & $\begin{array}{l}\text { Sept. } 8,1942 \\
\text { June } 5,1943\end{array}$ & $\overline{-}$ & $\begin{array}{l}35 \\
35\end{array}$ \\
\hline 1941 & 4,1941 & - & 69 & & & & \\
\hline
\end{tabular}


8-982.03. Brushy Creek watershed C near Riesel, Tex. (9)

Location.--Lat $31^{\circ} 31^{\prime} 1^{\prime \prime}$, long $96^{\circ} 53^{\prime} 34^{\prime \prime}$, at bridge on county road, 3.8 miles northeast of Riesel, McLennan County.

Drainage area. $--0.905 \mathrm{sq} \mathrm{ml}$.

Gage.--Recording. Datum of gage is $532.4 \mathrm{ft}$ above mean sea level.

Stage-discharge relation.--Defined by current-meter measurements.

Remarks.--Records furnished by U.S. Department of Agriculture, Agricultural Research Service, Blacklands Experimental Watershed at Riesel, Tex. Detalled information is avallable from the Riesel office regarding bas in shape and slope, type of soils, erosion conditions, watershed conditions (kind of soil cover that bear a relationship to hydrology, and rainfall. The drainage area is artificlally controlled, therefore no gage helghts are shown. Only annual (calendar year) peaks are shown.

\begin{tabular}{|c|c|c|c|c|c|c|c|}
\hline $\begin{array}{l}\text { Calen- } \\
\text { dar } \\
\text { year }\end{array}$ & Date & $\begin{array}{c}\text { Cage } \\
\text { helght } \\
\text { (feet) }\end{array}$ & $\begin{array}{c}\text { Discharge } \\
(\text { cfs })\end{array}$ & $\begin{array}{l}\text { Calen- } \\
\text { dar } \\
\text { year }\end{array}$ & Date & $\begin{array}{c}\text { Gage } \\
\text { helght } \\
\text { (feet) }\end{array}$ & $\begin{array}{c}\text { Discharge } \\
\text { (cfs) }\end{array}$ \\
\hline $\begin{array}{l}1939 \\
1940 \\
1941 \\
1942\end{array}$ & $\begin{array}{l}\text { June 19, } 1939 \\
\text { Nov. 22, } 1940 \\
\text { June 10, } 1941 \\
\text { Sept 8, } 1942\end{array}$ & $\begin{array}{l}- \\
- \\
-\end{array}$ & $\begin{array}{l}105 \\
485 \\
\\
514 \\
438\end{array}$ & $\begin{array}{l}1953 \\
1954 \\
1955\end{array}$ & $\begin{array}{l}\text { Mar. 12, } 1953 \\
\text { May 11, } 1954 \\
\text { Mar. 21, } 1955\end{array}$ & $\begin{array}{l}\overline{-} \\
\overline{-}\end{array}$ & $\begin{array}{l}309 \\
438 \\
117\end{array}$ \\
\hline 1943 & $\begin{array}{l}\text { sept. } \\
\text { June } 5,1943\end{array}$ & $\overline{-}$ & $\begin{array}{l}438 \\
216\end{array}$ & $\begin{array}{l}1956 \\
1957 \\
1958\end{array}$ & $\begin{array}{l}\text { May } 1,1956 \\
\text { Apr. } 19,1957\end{array}$ & - & $\begin{array}{l}123 \\
776\end{array}$ \\
\hline $\begin{array}{l}1949 \\
1950\end{array}$ & $\begin{array}{l}\text { June } 15,1949 \\
\text { Feb. } 12,1950\end{array}$ & - & $\begin{array}{r}88 \\
385\end{array}$ & $\begin{array}{l}1970 \\
1959 \\
1960\end{array}$ & $\begin{array}{l}\text { June 23, } 1959 \\
\text { Dec. } 7,1960\end{array}$ & $\overline{-}$ & $\begin{array}{l}467 \\
365 \\
152\end{array}$ \\
\hline $\begin{array}{l}1951 \\
1952\end{array}$ & $\begin{array}{ll}\text { Sept.13, } 1951 \\
\text { May 23, } 1952\end{array}$ & - & $\begin{array}{l}105 \\
210\end{array}$ & $\begin{array}{l}1961 \\
1962 \\
1963 \\
\end{array}$ & $\begin{array}{l}\text { Feb. 16, } 1961 \\
\text { June 4, } 1962 \\
\text { Apr. 26, } 1963\end{array}$ & $\begin{array}{l}- \\
- \\
\end{array}$ & $\begin{array}{r}204 \\
181 \\
18 \\
\end{array}$ \\
\hline
\end{tabular}

8-982.06. Brushy Creek watershed D near Riesel, Tex. (9)

Location. - - Lat $31^{\circ} 30^{\prime} 38^{\prime \prime}$, long $96^{\circ} 53^{\prime} 22^{\prime \prime}$, at bridge on county road, 3.2 miles northeast of Riesel, McLennan County.

Drainage area. $--1.73 \mathrm{sq} \mathrm{ml}$.

Gage.--Recording. Datum of gage is $518.8 \mathrm{ft}$ above mean sea level.

Stage-discharge relation.--Defined by current-meter measurements .

Remarks.--Records furnished by U.S. Department of Agriculture, Agricultural Research Service, Blacklands Experimental Watershed at Riesel, Tex. Detailed information is avallable from the Riesel office regarding bas in shape and slope, type of solls, erosion conditions, watershed conditions (kind of soll cover that bear a relationship to hydrology, and rainfall. The drainage area is artificlally controlled, therefore no gage heights are shown. Only annual (calendar year) peaks are shown.

\begin{tabular}{|c|c|c|c|c|c|c|c|}
\hline $\begin{array}{l}\text { Calen- } \\
\text { dar } \\
\text { year } \\
\end{array}$ & Date & $\begin{array}{c}\text { Qage } \\
\text { height } \\
\text { (feet) }\end{array}$ & $\begin{array}{c}\text { Discharge } \\
(\text { cfs })\end{array}$ & $\begin{array}{l}\text { Calen- } \\
\text { dar } \\
\text { year }\end{array}$ & Date & $\begin{array}{c}\text { Gage } \\
\text { helght } \\
\text { (feet) }\end{array}$ & $\begin{array}{c}\text { Discharge } \\
(\mathrm{cfs})\end{array}$ \\
\hline $\begin{array}{l}1938 \\
1939 \\
1940\end{array}$ & $\begin{array}{l}\text { Apr. 15, } 1938 \\
\text { June 19, } 1939 \\
\text { Nov. 22, } 1940\end{array}$ & $\begin{array}{l}- \\
-\end{array}$ & $\begin{array}{r}201 \\
90 \\
795\end{array}$ & $\begin{array}{l}1953 \\
1954 \\
1955\end{array}$ & $\begin{array}{ll}\text { May } 12,1953 \\
\text { May 11, } 1954 \\
\text { Mar. 21, } 1955\end{array}$ & $\begin{array}{l}- \\
-\end{array}$ & $\begin{array}{l}828 \\
806 \\
168\end{array}$ \\
\hline $\begin{array}{l}1941 \\
1942 \\
1943 \\
\end{array}$ & $\begin{array}{l}\text { June 10, } 1941 \\
\text { Sept. 8, } 1942 \\
\text { June } 5,1943\end{array}$ & $\begin{array}{l}- \\
-\end{array}$ & $\begin{array}{l}839 \\
515 \\
302\end{array}$ & $\begin{array}{l}1956 \\
1957 \\
1958\end{array}$ & $\begin{array}{l}\text { Nov. 4, } 1956 \\
\text { Apr. 19, } 1957 \\
\text { Sept.19, } 1958\end{array}$ & $\begin{array}{l}- \\
-\end{array}$ & $\begin{array}{r}134 \\
1,150 \\
\end{array}$ \\
\hline $\begin{array}{l}1949 \\
1950\end{array}$ & $\begin{array}{ll}\text { June } 15, & 1949 \\
\text { July } 15, & 1950\end{array}$ & - & $\begin{array}{l}235 \\
604\end{array}$ & $\begin{array}{l}1959 \\
1960\end{array}$ & $\begin{array}{l}\text { June 23, } 1959 \\
\text { Dec. 7, } 1960\end{array}$ & - & $\begin{array}{l}676 \\
269\end{array}$ \\
\hline $\begin{array}{l}1951 \\
1952 \\
\end{array}$ & $\begin{array}{l}\text { Sept.13, } 1951 \\
\text { May 23, } 1952\end{array}$ & - & $\begin{array}{l}571 \\
470 \\
\end{array}$ & $\begin{array}{l}1961 \\
1962 \\
1963 \\
\end{array}$ & $\begin{array}{l}\text { Feb. } 16,1961 \\
\text { June 4, } 1962 \\
\text { Apr. 26, } 1963\end{array}$ & $\begin{array}{l}- \\
- \\
\end{array}$ & $\begin{array}{r}402 \\
246 \\
11 \\
\end{array}$ \\
\hline
\end{tabular}


8-982.09. Brushy Creek watershed SW-14 near Riesel, Tex.(9)

Location.--Lat $31^{\circ} 28159^{\prime \prime}$, Iong $96^{\circ} 53^{\prime 2} 7^{\prime \prime}$, on Blacklands Experimental Watershed, 2 miles east of Riese1, McLennan County.

Drainage area. $-0.0047 \mathrm{sq} \mathrm{ml}$.

Gage.--Recording. Datum of gage is $541.8 \mathrm{ft}$ above mean sea level.

Stage-discharge relation.--Defined by theoretical rating for 3-foot H-flume.

Remarks.--Records furnished by U.S. Department of Agriculture, Agricultural

Research Serv1ce, Blacklands Experimental Watershed at Riesel, Tex. Deta1led

information is available from the Riesel office regarding basin shape and slope, type of solls, erosion condition, watershed conditions (kind of soll cover) that bear a relationship to hydrology, and rainfall. Only annual (calendar year) peaks are shown.

Peak stages and discharges

\begin{tabular}{|c|c|c|c|c|c|c|c|}
\hline $\begin{array}{l}\text { Calen- } \\
\text { dar } \\
\text { year } \\
\end{array}$ & Date & $\begin{array}{c}\text { Gage } \\
\text { helght } \\
\text { (feet) }\end{array}$ & $\begin{array}{c}\text { D1scharge } \\
\text { (cfs) }\end{array}$ & $\begin{array}{l}\text { Calen- } \\
\text { dar } \\
\text { year }\end{array}$ & Date & $\begin{array}{c}\text { Gage } \\
\text { nelght } \\
\text { (feet) }\end{array}$ & $\begin{array}{c}\text { Discharge } \\
\text { (cfs) }\end{array}$ \\
\hline $\begin{array}{l}1939 \\
1940\end{array}$ & $\begin{array}{lr}\text { May } 17, & 1939 \\
\text { July } 3, & 1940\end{array}$ & $\overline{-}$ & 14.1 & $\begin{array}{l}1942 \\
1943\end{array}$ & $\begin{array}{l}\text { June } 11,1942 \\
\text { June } 5,1943\end{array}$ & $\overline{-}$ & $\begin{array}{l}13 \\
9.6\end{array}$ \\
\hline 1941 & June 10,1941 & - & 12 & & & & \\
\hline
\end{tabular}

\section{8-982.12. Brushy Creek watershed SW-12 near Riesel, Tex.(9)}

Location.--Lat $31^{\circ} 28^{\prime} 48^{\prime \prime}$, long $96^{\circ} 52^{\prime} 59^{\prime \prime}$, on Blacklands Experimental Watershed, 2 miles east of Riesel, McLennan County.

Drainage area. $--0.0046 \mathrm{sq} \mathrm{ml}$.

Gage.--Recording. Datum of gage is $531.7 \mathrm{ft}$ above mean sea level.

Stage-discharge relation.--Defined by theoretical rating for 3-foot $\mathrm{H}$-flume.

Remarks.--Records furnished by U.S. Department of Agriculture, Agricultural Research Service, Blacklands Experimental Watershed at Riesel, Tex. Detalled information is available from the Riesel office regarding basin shape and slope, type of solls, erosion condition, watershed conditions (kind of soil cover that bear a relationship to hydrology, and rainfall. Only annual (calendar year) peaks are shown.

Peak stages and discharges

\begin{tabular}{|c|c|c|c|c|c|c|c|}
\hline $\begin{array}{l}\text { Calen- } \\
\text { dar } \\
\text { year } \\
\end{array}$ & Date & $\begin{array}{c}\text { Gage } \\
\text { helght } \\
\text { (reet) }\end{array}$ & $\begin{array}{c}\text { D1scharge } \\
(\mathrm{cfs})\end{array}$ & $\begin{array}{l}\text { Calen- } \\
\text { dar } \\
\text { year }\end{array}$ & Date & $\begin{array}{c}\text { Gage } \\
\text { helght } \\
\text { (feet) }\end{array}$ & $\begin{array}{c}\text { D1scharge } \\
(\mathrm{cfs})\end{array}$ \\
\hline $\begin{array}{l}1938 \\
1939 \\
1940\end{array}$ & $\begin{array}{l}\text { Feb. } 16,1938 \\
\text { Nov. } 2 \overline{4}, 1940\end{array}$ & - & $\begin{array}{l}1.3 \\
0 \\
2.8\end{array}$ & $\begin{array}{l}1952 \\
1953 \\
1954 \\
1955\end{array}$ & $\begin{array}{l}\text { Dec. } 30,1952 \\
\text { Mar. 12, } 1953 \\
\text { May 11, } 1954 \\
\text { Apr. 21, } 1955\end{array}$ & $\begin{array}{l}- \\
- \\
-\end{array}$ & $\begin{array}{r}0.6 \\
6.5 \\
.1 \\
.5\end{array}$ \\
\hline $\begin{array}{l}1941 \\
1942\end{array}$ & $\begin{array}{l}\text { June 10, } 1941 \\
\text { June } 11,1942\end{array}$ & - & $\begin{array}{l}10 \\
5.4\end{array}$ & 1956 & 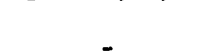 & - & 0 \\
\hline 1943 & Mar. 24,1943 & - & .1 & $\begin{array}{l}1957 \\
1958\end{array}$ & Apr. 19, 1957 & - & 10 \\
\hline $\begin{array}{l}1948 \\
1949\end{array}$ & $\begin{array}{l}\text { Apr. 25, } 1948 \\
\text { Apr. 27, } 1949\end{array}$ & - & $\begin{array}{l}.3 \\
.1\end{array}$ & $\begin{array}{l}1959 \\
1960\end{array}$ & $\begin{array}{l}\text { June 23, } 1959 \\
\text { Jan. 13, } 1960\end{array}$ & - & $\begin{array}{l}.4 .1 \\
1.3\end{array}$ \\
\hline 1950 & Feb. 12,1950 & . & 6.8 & & & & \\
\hline 1951 & Sept.13, 1951 & - & (a) & $\begin{array}{l}1961 \\
1962 \\
1963\end{array}$ & $\begin{array}{l}\text { Feb. 16, } 1961 \\
\text { June 9, } 1962\end{array}$ & - & $\begin{array}{c}1.8 \\
12 \\
0\end{array}$ \\
\hline
\end{tabular}

a Less than 0.1 cfs. 
8-982.15. Brushy Creek watershed Y-10 near R1ese1, Tex. (9)

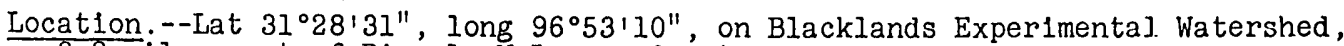
$2.2 \mathrm{mlles}$ east of Riesel, McLennan County.

Drainage area. $--0.0291 \mathrm{sq} \mathrm{mi;} 0.0328 \mathrm{sq} \mathrm{mi}$ prior to Jan. 1, 1956.

Gage.--Recording. Datum of gage is $539 \mathrm{ft}$ above mean sea level.

Stage-discharge relation.--Defined by theoretical rating for 6-foot Parshall flume, modified with weir for measuring low flows.

Remarks.--Records furnished by U.S. Department of Agriculture, Agricultural Research Service, Blacklands Experimental Watershed at Riesel, Tex. Detailed information is available from the Riesel office regarding basin shape and slope, type of solls, erosion condition, watershed conditions (kind of soll cover that bear a relationship to hydrology, and rainfall. Only annual (calendar year) peaks are shown.

\begin{tabular}{l|c|c|c||c|c|c|c}
\hline $\begin{array}{c}\text { Calen- } \\
\text { dar } \\
\text { year }\end{array}$ & Date & $\begin{array}{c}\text { Gage } \\
\text { height } \\
\text { (feet) }\end{array}$ & $\begin{array}{c}\text { Discharge } \\
\text { (cfs) }\end{array}$ & $\begin{array}{c}\text { Calen- } \\
\text { dar } \\
\text { year }\end{array}$ & Date & $\begin{array}{c}\text { Gage } \\
\text { he1ght } \\
\text { (feet) }\end{array}$ & $\begin{array}{c}\text { D1scharge } \\
\text { (cfs) }\end{array}$ \\
\hline 1939 & May 20, 1939 & - & 12 & 1952 & May 23, 1952 & - & 24 \\
1940 & Nov. 22, 1940 & - & 52 & 1953 & May 12, 1953 & - & 19 \\
1941 & June 10, 1941 & - & 73 & 1954 & May 11, 1954 & - & 20 \\
1942 & June 11, 1942 & - & 59 & 1955 & Mar. 21, 1955 & - & 35 \\
1943 & June 5, 1943 & - & 21 & 1956 & Nov. 4, 1956 & - & 2.4 \\
1946 & May 12, 1946 & - & 34 & 1957 & Apr. 19, 1957 & - & 70 \\
1947 & Mar. 18, 1947 & - & 6.8 & 1958 & May 3, 1958 & - & 6.0 \\
1948 & Apr. 25, 1948 & - & 1959 & June 23, 1959 & - & 13 \\
1949 & July 4, 1949 & - & 20 & 1960 & June 26, 1960 & - & 7.3 \\
1950 & Feb. 12, 1950 & - & 19 & 1961 & June 25, 1961 & - & 11 \\
1951 & June 16, 1951 & - & 2.5 & 1962 & June 9, 1962 & - & 7.3 \\
\hline
\end{tabular}

8-982.18. Brushy Creek watershed Y-6 near Riese1, Tex. (9)

Location:--Lat $31^{\circ} 28^{\prime} 26^{\prime \prime}$, long 96 $53^{\prime} 09^{\prime \prime}$, on Blacklands Experimental Watershed, 2.2 miles east of Riesel, McLennan County.

Drainage area. $--0.0255 \mathrm{sq} \mathrm{mi} ; 0.0327 \mathrm{sq} \mathrm{mi}$ prior to Jan. 1, 1956.

Gage.--Recording. Datum of gage is $538 \mathrm{ft}$ above mean sea level.

Stage-discharge relation.--Defined by theoretical rating for 6-foot Parshall flume, modified with weir for measuring low flows.

Remarks.--Records furnished by U.S. Department of Agriculture, Agricultural Research Service, Blacklands Experimental Watershed at Riesel, Tex. Detailed information is avallable from the Riesel offlce regarding basin shape and slope, type of solls, erosion condition, watershed conditions (kind of soll cover that bear a relationship to hydrology, and rainfall. Only annual (calendar year) peaks are shown.

\begin{tabular}{|c|c|c|c|c|c|c|c|}
\hline $\begin{array}{l}\text { Calen- } \\
\text { dar } \\
\text { year } \\
\end{array}$ & Date & $\begin{array}{c}\text { Gage } \\
\text { he1ght } \\
\text { (feet) }\end{array}$ & $\begin{array}{l}\text { Discharge } \\
\text { (cfs) }\end{array}$ & $\begin{array}{l}\text { Calen-: } \\
\text { dar } \\
\text { year }\end{array}$ & Date & $\begin{array}{c}\text { Gage } \\
\text { he1ght } \\
\text { (feet) }\end{array}$ & $\begin{array}{c}\text { Discharge } \\
\text { (cfs) }\end{array}$ \\
\hline $\begin{array}{l}1939 \\
1940\end{array}$ & $\begin{array}{lll}\text { May } & 17, & 1939 \\
\text { Nov. } & 22, & 1940\end{array}$ & $\overline{-}$ & $\begin{array}{l}16 \\
55\end{array}$ & $\begin{array}{l}1953 \\
1954 \\
1955\end{array}$ & $\begin{array}{lll}\text { May } 12,1953 \\
\text { May } 11, & 1954 \\
\text { Mar. } & 21, & 1955\end{array}$ & - & $\begin{array}{l}11 \\
11 \\
19\end{array}$ \\
\hline 1941 & June 10, 1941 & - & 80 & & & & \\
\hline $\begin{array}{l}1942 \\
1943 \\
\end{array}$ & $\begin{array}{l}\text { Sept. } 8,1942 \\
\text { June } 5,1943\end{array}$ & - & & $\begin{array}{l}1956 \\
1957\end{array}$ & $\begin{array}{l}\text { Nov. } 4,1956 \\
\text { Apr. } 19,1957\end{array}$ & - & $\begin{array}{l}2.8 \\
27\end{array}$ \\
\hline $\begin{array}{l}1948 \\
1949 \\
1950\end{array}$ & $\begin{array}{ll}\text { Apr. } 25, & 1948 \\
\text { Juiy } 4, & 1949 \\
\text { Feb. 12, } & 1950\end{array}$ & - & $\begin{array}{l}12 \\
20 \\
20\end{array}$ & $\begin{array}{l}1958 \\
1959 \\
1960\end{array}$ & $\begin{array}{l}\text { Oct. 21, } 1958 \\
\text { June 23, } 1959 \\
\text { Dec. 8, } 1960\end{array}$ & - & $\begin{array}{l}2.3 \\
17 \\
3.3\end{array}$ \\
\hline $\begin{array}{l}1951 \\
1952 \\
\end{array}$ & $\begin{array}{l}\text { Sept.13, } 1951 \\
\text { May } 23,1952\end{array}$ & - & $\begin{array}{l}2.3 \\
11\end{array}$ & $\begin{array}{l}1961 \\
1962 \\
1963 \\
\end{array}$ & $\begin{array}{l}\text { June } 15,1961 \\
\text { June } 9,1962\end{array}$ & - & $\begin{array}{r}14 \\
16 \\
0\end{array}$ \\
\hline
\end{tabular}


Location.--Lat $31^{\circ} 28^{\prime} 30^{\prime \prime}$, long $96^{\circ} 52^{\prime} 54^{\prime \prime}$, on Blacklands Experimental Watershed, $2.4 \mathrm{~m} 1 \mathrm{les}$ east of Riesel, McLennan County.

Drainage area. $--0.125 \mathrm{sq} \mathrm{ml}$.

Goge.--Recording. Datum of gage is $524 \mathrm{ft}$ above mean sea level.

Stage-discharge relation.--Defined by theoretical rating for 10-foot Parshall flume, modified with weir for measuring low flows.

Remarks.--Records furnished by U.S. Department of Agriculture, Agricultural Research Service, Blacklands Experimental Watershed at Riesel, Tex. Detalled information is avaliable from the Rlesel office regarding basin shape and slope, type of solls, erosion condition, watershed conditions (kind of soll cover) that bear a relationship to hydrology, and rainfall. Only annual (calendar year) peaks are shown.

\begin{tabular}{|c|c|c|c|c|c|c|c|}
\hline $\begin{array}{l}\text { Calen- } \\
\text { dar } \\
\text { year }\end{array}$ & Date & $\begin{array}{c}\text { aage } \\
\text { helght } \\
\text { (feet) }\end{array}$ & $\begin{array}{c}\text { D1scharge } \\
(\mathrm{cfs})\end{array}$ & $\begin{array}{l}\text { Calen- } \\
\text { dar } \\
\text { year }\end{array}$ & Date & $\begin{array}{c}\text { Gage } \\
\text { helght } \\
\text { (feet) }\end{array}$ & $\begin{array}{c}\text { Discharge } \\
(\mathrm{cfs})\end{array}$ \\
\hline $\begin{array}{l}1939 \\
1940 \\
1941 \\
1942 \\
1943 \\
\end{array}$ & $\begin{array}{l}\text { May 20, } 1939 \\
\text { Nov. 22, } 1940 \\
\text { June } 10,1941 \\
\text { Sept. 8, } 1942 \\
\text { June } 5,1943\end{array}$ & $\begin{array}{l}- \\
- \\
- \\
-\end{array}$ & $\begin{array}{r}46 \\
187 \\
251 \\
197 \\
24\end{array}$ & $\begin{array}{l}1952 \\
1953 \\
1954 \\
1955 \\
1956\end{array}$ & $\begin{array}{l}\text { May 23, } 1952 \\
\text { May 12, } 1953 \\
\text { May 11, } 1954 \\
\text { Mar. 21, } 1955 \\
\text { Nov. 4, } 1956\end{array}$ & $\begin{array}{l}- \\
- \\
-\end{array}$ & $\begin{array}{l}31 \\
46 \\
50 \\
83 \\
9.7\end{array}$ \\
\hline $\begin{array}{l}1946 \\
1947 \\
1948 \\
1949 \\
1950 \\
1951\end{array}$ & $\begin{array}{l}\text { May 12, } 1946 \\
\text { Mar. 18, } 1947 \\
\text { Apr. 25, } 1948 \\
\text { July } 4,1949 \\
\text { Feb. 12, } 1950 \\
\text { Sept.13, } 1951\end{array}$ & $\begin{array}{l}- \\
- \\
- \\
-\end{array}$ & $\begin{array}{r}111 \\
23 \\
48 \\
72 \\
76 \\
\\
\text { (a) } \\
\end{array}$ & $\begin{array}{l}1957 \\
1958 \\
1959 \\
1960 \\
1961 \\
1962 \\
1963\end{array}$ & 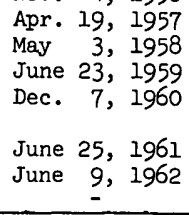 & $\begin{array}{l}- \\
- \\
- \\
- \\
-\end{array}$ & $\begin{array}{r}203 \\
12 \\
64 \\
15 \\
27 \\
53 \\
0 \\
\end{array}$ \\
\hline
\end{tabular}

8-982.24. Brushy Creek watershed Y-8 near Riesel, Tex.(9)

Location.--Lat $31^{\circ} 28^{\prime} 22^{\prime \prime}$, long $96^{\circ} 52^{\prime} 54^{\prime \prime}$, on Blacklands Experimental Watershed, 2.5 miles east of Riese1, McLennan County.

Drainage area. $--0.0325 \mathrm{sq} \mathrm{mi}$.

Gage.--Recording. Datum of gage is $537 \mathrm{ft}$ above mean sea level.

Stage-discharge relation.--Defined by theoretical rating for 6-foot Parshall flume, modifled with weir for measuring low flows.

Remarks.--Records furnished by U.S. Department of Agriculture, Agricultural Research Service, Blacklands Experimental Watershed at Riesel, Tex. Detalled information is avallable from the Riesel offlce regarding bas in shape and slope, type of solis, erosion condition, watershed conditions (kind of soil cover that bear a relationship to hydrology, and rainfall. Only annual (calendar year) peaks are shown.

\begin{tabular}{|c|c|c|c|c|c|c|c|}
\hline $\begin{array}{l}\text { Calen- } \\
\text { dar } \\
\text { year }\end{array}$ & Date & $\begin{array}{l}\text { Cage } \\
\text { helight } \\
\text { (feet) }\end{array}$ & $\begin{array}{c}\text { D1scharge } \\
\text { (cfs) }\end{array}$ & $\begin{array}{l}\text { Calen- } \\
\text { dar } \\
\text { year }\end{array}$ & Date & $\begin{array}{c}\text { Gage } \\
\text { helght } \\
\text { (feet) }\end{array}$ & $\begin{array}{c}\text { D1scharge } \\
\text { (cfs) }\end{array}$ \\
\hline $\begin{array}{l}1939 \\
1940\end{array}$ & $\begin{array}{ll}\text { May } 18, & 1939 \\
\text { Nov. } 22, & 1940\end{array}$ & - & $\begin{array}{l}13 \\
52\end{array}$ & $\begin{array}{l}1953 \\
1954 \\
1955\end{array}$ & $\begin{array}{lll}\text { May } 12, & 1953 \\
\text { May } 11, & 1954 \\
\text { Mar. 21, } & 1955\end{array}$ & $\begin{array}{l}\text { - } \\
\text { - }\end{array}$ & $\begin{array}{l}20 \\
16 \\
28\end{array}$ \\
\hline $\begin{array}{l}1941 \\
1942\end{array}$ & $\begin{array}{l}\text { June } 10,1941 \\
\text { Sept. } 8,1942\end{array}$ & - & $\begin{array}{l}69 \\
60\end{array}$ & 1956 & 1,1956 & - & .8 \\
\hline 943 & June 5,1943 & - & 15 & $\begin{array}{l}1957 \\
1958\end{array}$ & Apr. 19,1957 & - & \\
\hline $\begin{array}{l}1949 \\
1950\end{array}$ & $\begin{array}{l}\text { July 4, } 1949 \\
\text { Feb. } 12,1950\end{array}$ & - & $\begin{array}{l}17 \\
14\end{array}$ & $\begin{array}{l}1959 \\
1960\end{array}$ & $\begin{array}{l}\text { June 23, } 1959 \\
\text { Dec. } 7,1960\end{array}$ & - & 57.0 \\
\hline $\begin{array}{l}1951 \\
1952\end{array}$ & $\begin{array}{ll}\text { June } & 16, \\
\text { May } & 1951 \\
1952\end{array}$ & - & $15^{.4}$ & $\begin{array}{l}1961 \\
1962 \\
1963 \\
\end{array}$ & $\begin{array}{cc}\text { Feb. } & 5,1961 \\
\text { June } & 9,1962 \\
& -\end{array}$ & $\begin{array}{l}- \\
-\end{array}$ & $\begin{array}{c}5.0 \\
39 \\
0\end{array}$ \\
\hline
\end{tabular}




\section{BRAZOS RIVER BASIN}

8-982.27. Brushy Creek watershed Y-2 near Riese1, Tex. (9)

Location.--Lat $31^{\circ} 28^{\prime} 30^{\prime \prime}$, long $96^{\circ} 52^{\prime} 46^{\prime \prime}$, on Blacklands Experimental Watershed, 2.5 miles east of Rlesel, McLennan County.

Drainage area. $--0.206 \mathrm{sq} \mathrm{ml}$.

Gage.--Recording. Datum of gage is $518 \mathrm{ft}$ above mean sea level.

Stage-discharge relation.--Defined by theoretical rating for $15^{\circ}$-foot Parshall fiume, modified with weir for measuring low flows.

Remarks.--Records furnished by U.S. Department of Agriculture, AgriculturaI Research Service, Blacklands Experimental Watershed at Riese1, Tex. Detailed information is available from the Riesel office regarding basin shape and slope, type of soils, erosion condition, watershed conditions (kind of soll cover) that bear a relationship to hydrology, and rainfall. Only annual (calendar year) peaks are shown.

Peak stages and discharges

\begin{tabular}{|c|c|c|c|c|c|c|c|}
\hline $\begin{array}{l}\text { Calen- } \\
\text { dar } \\
\text { year }\end{array}$ & Date & $\begin{array}{c}\text { Cage } \\
\text { he1ght } \\
\text { (feet) }\end{array}$ & $\begin{array}{c}\text { D1scharge } \\
(\mathrm{cfs})\end{array}$ & $\begin{array}{l}\text { Calen- } \\
\text { dar } \\
\text { year }\end{array}$ & Date & $\begin{array}{c}\text { Gage } \\
\text { he1ght } \\
\text { (feet) }\end{array}$ & $\begin{array}{c}\text { D1scharge } \\
(\mathrm{cfs})\end{array}$ \\
\hline $\begin{array}{l}1939 \\
1940 \\
1941 \\
1942 \\
1943 \\
1944 \\
1945 \\
1946 \\
1947 \\
1948 \\
1949 \\
1950\end{array}$ & $\begin{array}{l}\text { May } 20,1939 \\
\text { Nov. } 22,1940 \\
\text { June 10, } 1941 \\
\text { Sept. 8, } 1942 \\
\text { June } 5,1943 \\
\text { May 1, } 1944 \\
\text { Mar. } 3,1945 \\
\text { May 12, } 1946 \\
\text { Mar. 18, } 1947 \\
\text { Apr. 25, } 1948 \\
\text { July 4, } 1949 \\
\text { Feb. 12, } 1950\end{array}$ & $\begin{array}{l}- \\
- \\
- \\
- \\
- \\
- \\
- \\
- \\
- \\
-\end{array}$ & $\begin{array}{r}71 \\
291 \\
406 \\
327 \\
35 \\
542 \\
170 \\
\\
208 \\
45 \\
110 \\
118 \\
126\end{array}$ & $\begin{array}{l}1951 \\
1952 \\
1953 \\
1954 \\
1955 \\
1956 \\
1957 \\
1958 \\
1959 \\
1960 \\
1961 \\
1962 \\
1963\end{array}$ & 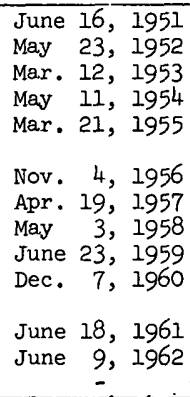 & $\begin{array}{l}\overline{-} \\
\overline{-} \\
- \\
- \\
- \\
- \\
-\end{array}$ & $\begin{array}{r}(\mathrm{a}) \\
56 \\
74 \\
64 \\
138 \\
\\
13 \\
344 \\
17 \\
106 \\
23 \\
\\
35 \\
120 \\
0\end{array}$ \\
\hline
\end{tabular}

a Less than 0.1 cfs.

8-982.3. Brushy Creek watershed Y-7 near Riesel, Tex.(9)

Location.--Lat $31^{\circ} 28^{\prime} 08^{\prime \prime}$, long $96^{\circ} 52^{\prime} 49^{\prime \prime}$, on Blacklands Experimental Watershed, 2.5 miles east of Riesel, McLennan County.

Drainage area $--0.0625 \mathrm{sq} \mathrm{mi}$.

Gage.--Recording. Datum of gage is $544 \mathrm{ft}$ above mean sea level.

Stage-discharge relation.--Defined by theoretical rating for 6-foot Parshall flume, modified with weir for measuring low flows.

Remarks.--Records furnished by U.S. Department of Agriculture, Agricultural Research Service, Blacklands Experimental Watershed at Riesel, Tex. Detalled information is available from the Riesel office regarding basin shape and slope, type of solls, erosion condition, watershed conditions (kind of soll cover) that bear a relationship to hydrology, and rainfall. Only annual (calendar year) peaks are shown.

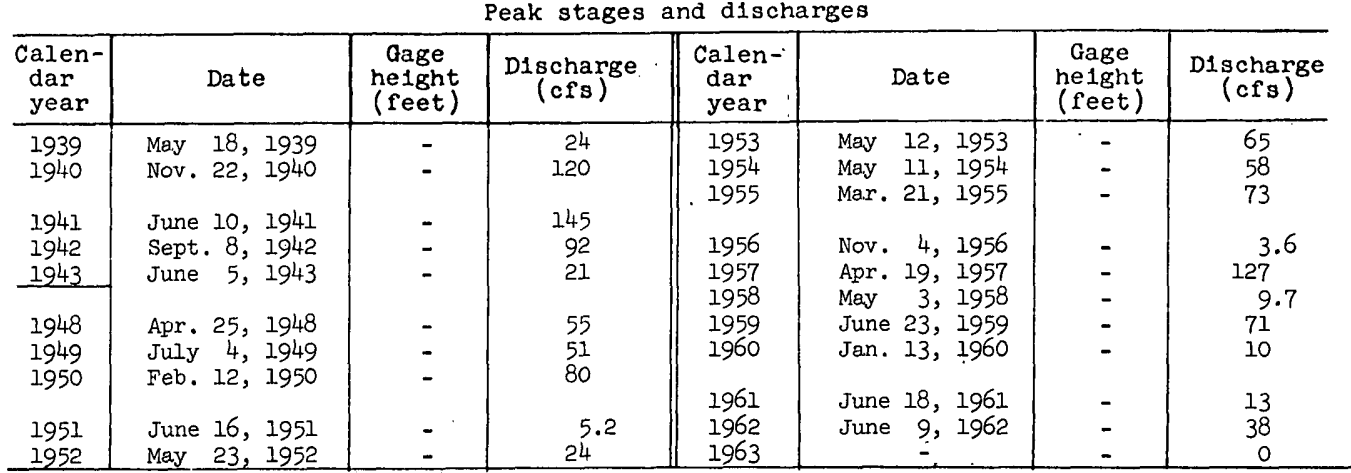


8-982.33. Brushy Creek watershed SW-7 near Riesel, Tex. (9)

Location.--Lat $31^{\circ} 28^{\prime} 11^{\prime \prime}$, long $96^{\circ} 52^{\prime} 59^{\prime \prime}$, on Blacklands Experimental Watershed, 2 miles east of Riesel, McLennan County.

Drainage area. $-0.0049 \mathrm{sq} \mathrm{ml}$.

Gage.--Recording. Datum of gage 1s $552.6 \mathrm{ft}$ above mean sea level.

Stage-discharge relation.--Defined by theoretical rating for 3-foot H-flume.

Remarks.--Records furnished by U.S. Department of Agriculture, Agricultural

Research Service, Blacklands Experimental Watershed at Riesel, Tex. Detalled

Information is avallable from the Riesel offlce regarding basin shape and slope, type of solls, erosion condition, watershed conditions (kind of soll cover that bear a relationship to hydrology, and rainfall. Only annual (calendar year) peaks are shown.

Peak stages and discharges

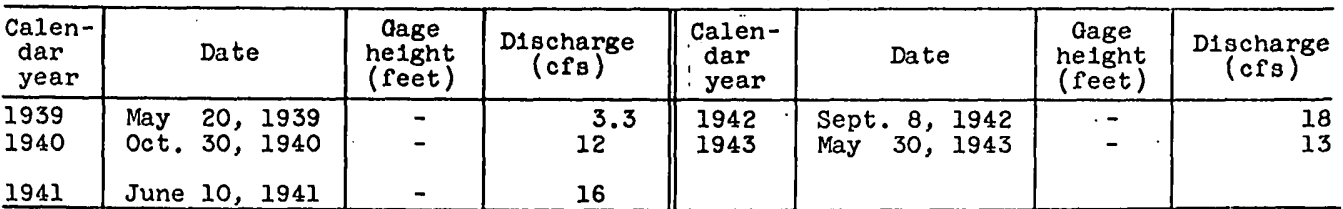

8-982.36. Brushy Creek watershed SW-13 near Riesel, Tex. (9)

Location.--Lat $31^{\circ} 28^{\prime} 41^{\prime \prime}$, long $96^{\circ} 52^{\prime} 48^{\prime \prime}$, on Blacklands Experimental Watershed, 2 miles east of Riesel, McLennan County.

Drainage area. $--0.0050 \mathrm{sq} \mathrm{ml}$.

Gage.--Recording. Datum of gage is $535.8 \mathrm{ft}$ above mean sea level.

Stage-discharge relation.--Defined by theoretical rating for 3 -foot $\mathrm{H}$-flume.

Remarks.--Records furnished by U.S. Department of Agriculture, Agricultural Research Service, Blacklands Experimental Watershed at Riesel, Tex. Detalled information is avallable from the Riesel office regarding basin shape and slope, type of solls, erosion condition, watershed conditions ( $k$ ind of soll cover) that bear a relationship to hydrology, and rainfall. Only annual (calendar year) peaks are shown.

Peak stages and discharges

\begin{tabular}{|c|c|c|c|c|c|c|c|c|}
\hline $\begin{array}{l}\text { Calen- } \\
\text { dar } \\
\text { year }\end{array}$ & & Date & $\begin{array}{c}\text { Cage } \\
\text { height } \\
\text { (feet) }\end{array}$ & $\begin{array}{c}\text { Discharge } \\
(\text { crs })\end{array}$ & $\begin{array}{l}\text { Calen- } \\
\text { dar } \\
\text { year }\end{array}$ & Date & $\begin{array}{c}\text { Gage } \\
\text { helght } \\
\text { (feet) }\end{array}$ & $\begin{array}{c}\text { D1scharge } \\
(\mathrm{cfs})\end{array}$ \\
\hline $\begin{array}{l}1939 \\
1940\end{array}$ & $\begin{array}{l}\text { May } \\
\text { Oct. }\end{array}$ & $\begin{array}{ll}17, & 1939 \\
31, & 1940\end{array}$ & - & $22^{4.9}$ & $\begin{array}{l}1942 \\
1943\end{array}$ & $\begin{array}{l}\text { June } 11,1942 \\
\text { June } 5,1943\end{array}$ & - & $\begin{array}{r}18 \\
5.5\end{array}$ \\
\hline 1941 & June & $10,1941-$ & $\therefore$. & 11 & & & & \\
\hline
\end{tabular}

8-982.39. Brushy Creek watershed Y near Riesel, Tex. (9)

Location.--Lat $31^{\circ} 28^{\prime} 36^{\prime \prime}$, long $96^{\circ} 52^{\prime} 36^{\prime \prime}$, on Blacklands Experimental Watershed, 2.7 miles east of Riesel, McLennan County.

Dralnage area. $-0.483 \mathrm{sq} \mathrm{ml}$.

Gage.--Recording. Datum of gage is $505.8 \mathrm{ft}$ above mean sea level.

Stage-d1scharge relation.--Defined by current-meter measurements.

Remarks.--Records furnished by U.S. Department of Agriculture, Agricultural Research Service, Blacklands Experimental Watershed at Riesel, Tex. Detalled information is avallable from the Riesel office regarding basin shape and slope type of solis, erosion condition, watershed conditions (kind of soll cover that bear a relationship to hydrology, and rainfall. Only annual (calendar year) peaks are shown. 
BRAZOS RIVER BASIN

Peak stages and dlscharges of Brushy Creek watershed Y near Rlesel, Tex.

\begin{tabular}{|c|c|c|c|c|c|c|c|}
\hline $\begin{array}{l}\text { Calen- } \\
\text { dar } \\
\text { year }\end{array}$ & Date & $\begin{array}{c}\text { Gage } \\
\text { helght } \\
\text { (feet) }\end{array}$ & $\begin{array}{c}\text { Discharge } \\
\text { (cfs) }\end{array}$ & $\begin{array}{l}\text { Calen- } \\
\text { dar } \\
\text { year }\end{array}$ & Date & $\begin{array}{c}\text { Gage } \\
\text { he1ght } \\
\text { (feet) }\end{array}$ & $\begin{array}{c}\text { Discharge } \\
(\mathrm{cfs})\end{array}$ \\
\hline $\begin{array}{l}1938 \\
1939 \\
1940 \\
1941 \\
1942 \\
1943 \\
\end{array}$ & 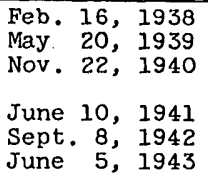 & $\begin{array}{l}- \\
- \\
- \\
-\end{array}$ & $\begin{array}{r}231 \\
122 \\
707 \\
760 \\
570 \\
78\end{array}$ & $\begin{array}{l}1951 \\
1952 \\
1953 \\
1954 \\
1955 \\
1956\end{array}$ & $\begin{array}{lll}\text { June } 16, & 1951 \\
\text { May } 23, & 1952 \\
\text { Mar. } 12,1953 \\
\text { May } 11,1954 \\
\text { Mar. } 21,1955 \\
\text { Nov. } 4,1956\end{array}$ & $\begin{array}{l}- \\
- \\
- \\
-\end{array}$ & $\begin{array}{l}6.2 \\
122 \\
143 \\
137 \\
290 \\
9.3\end{array}$ \\
\hline $\begin{array}{l}1946 \\
1947 \\
1948 \\
1949 \\
1950\end{array}$ & $\begin{array}{lr}\text { May } 12,1946 \\
\text { Mar. } 18,1947 \\
\text { Apr. } 25,1948 \\
\text { July } 4,1949 \\
\text { Feb. } 12,1950\end{array}$ & $\begin{array}{l}- \\
- \\
- \\
-\end{array}$ & $\begin{array}{l}551 \\
100 \\
212 \\
312 \\
402\end{array}$ & $\begin{array}{l}1957 \\
1958 \\
1959 \\
1960 \\
1961 \\
1962 \\
1963\end{array}$ & 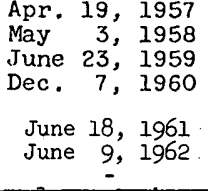 & $\begin{array}{l}- \\
- \\
- \\
- \\
-\end{array}$ & $\begin{array}{r}791 \\
34 \\
178 \\
47 \\
87 \\
221 \\
0 \\
\end{array}$ \\
\hline
\end{tabular}

8-982.42. Brushy Creek watershed G near Riesel, Tex. (9)

Location.--Iat $31^{\circ} 28^{\prime} 59^{\prime \prime}$, long $96^{\circ} 52^{\prime} 06^{\prime \prime}, 3.2$ miles east of Riesel, McLennan County.

Drainage area. $-6.84 \mathrm{sq} \mathrm{ml}$.

Gage.--Recording, with auxiliary slope recorder for high flows. Datum of gage is $478 \mathrm{ft}$ above mean sea level.

Stage-discharge relation.--Defined by current-meter measurements .

Remarks.--Records furnished by U.S. Department of Agriculture, Agricultural Research Service, Blacklands Experimental Watershed at Riesel, Tex. Detalled information is available from the Riesel office regarding bas in shape and slope, type of solls, erosion conditions, watershed conditions (kind of soil cover that bear a relationship to hydrology, and rainfall. Stage-discharge relation is affected by rate of change in stage, and peak stage occurred from 5 to 30 minutes after peak discharge. Only annual (calendar year) peaks are shown.

Peak stages and discharges

\begin{tabular}{|c|c|c|c|c|c|c|c|}
\hline $\begin{array}{l}\text { Calen- } \\
\text { dar } \\
\text { year }\end{array}$ & Date & $\begin{array}{c}\text { Gage } \\
\text { helght } \\
\text { (feet) }\end{array}$ & $\begin{array}{l}\text { Discharge } \\
\text { (cfs) }\end{array}$ & $\begin{array}{l}\text { Calen- } \\
\text { dar } \\
\text { year }\end{array}$ & Date & $\begin{array}{c}\text { Gage } \\
\text { helght } \\
\text { (feet) }\end{array}$ & $\begin{array}{c}\text { Discharge } \\
\text { (cfs) }\end{array}$ \\
\hline \multirow{5}{*}{$\begin{array}{l}1938 \\
1939 \\
1940 \\
1941 \\
1942\end{array}$} & \multirow{5}{*}{ 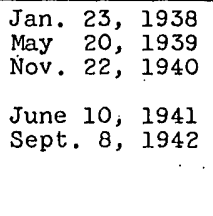 } & \multirow{5}{*}{$\begin{array}{l}6.08 \\
2.84 \\
8.48 \\
7.94 \\
7.87\end{array}$} & \multirow{5}{*}{$\begin{array}{r}795 \\
88 \\
1,850 \\
1,500 \\
1,100\end{array}$} & 1943 & June 5,1943 & \multirow{5}{*}{$\begin{array}{c}3.80 \\
6.96 \\
9.00 \\
6.38 \\
=-=- \\
- \\
-\end{array}$} & \multirow{5}{*}{$\begin{array}{r}707 \\
1,680 \\
680 \\
1,020 \\
441 \\
0\end{array}$} \\
\hline & & & & & & & \\
\hline & & & & $\begin{array}{l}1958 \\
1959\end{array}$ & $\begin{array}{l}\text { Sept.19, } 1958 \\
\text { June } 23,1959\end{array}$ & & \\
\hline & & & & 1960 & Dec. 7,1960 & & \\
\hline & & & & $\begin{array}{l}1961 \\
1962 \\
1963\end{array}$ & $\begin{array}{l}\text { Feb. } 5,1961 \\
\text { June } 9,1962\end{array}$ & & \\
\hline
\end{tabular}

8-982.45. Brushy Creek watershed Z near Riesel, Tex. (9)

Location. - - Lat $31^{\circ} 28^{\prime} 08^{\prime \prime}$, long $96^{\circ} 51^{\prime} 44^{\prime \prime}$, at bridge on county road, 3.7 miles southeast of Riesel; McLennan County.

Drainage area. $--0.484 \mathrm{sq} \mathrm{ml}$.

Gage.--Recording. Datum of gage is $492.0 \mathrm{ft}$ above mean sea level.

Stage-discharge relation.--Defined by current-meter measurements .

Remarks.--Records furnished by U.S. Department of Agriculture, Agricultural

Research Service, Blacklands Experimental Watershed at Riesel, Tex. Detalled information is avallable from the Riesel offlce regarding bas in shape and slope, type of solls, erosion conditions, watershed conditions (kind of soll cover that bear a relationship to hvdrology, and rainfall. The drainage area is artificlally controlled, therefore no gage heights are shown. Only annual (calendar year) peaks are shown.

Peak stages and discharges

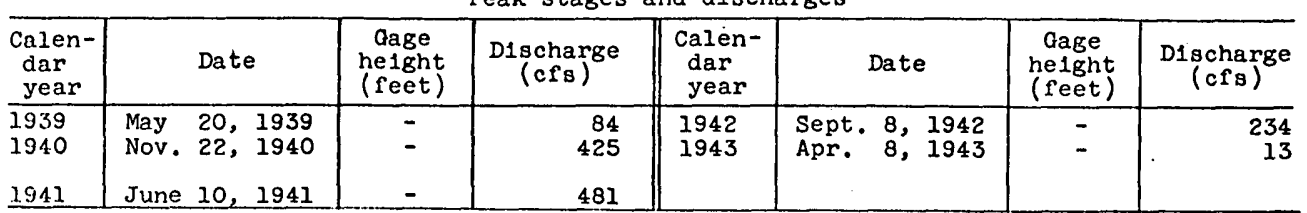


8-982.48. Brushy Creek watershed V, near Riesel, Tex. (9)

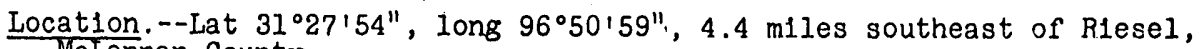
McLennan County.

Dra1nage area. $--9.16 \mathrm{sq} \mathrm{ml}$.

Gage.--Recording, with auxiliary slope recorder for high flows. Datum of gage Is $453.4 \mathrm{ft}$ above mean sea level.

Stage-discharge relation.--Defined by current-meter measurements.'

Remarks.--Records furnished by U.S. Department of Agriculture, Agricultural Research Service, Blacklands Experimental Watershed at Riesel, Tex. Deta1led information is available from the Riesel offlce regarding basin shape and slope, type of solls, erosion conditions, watershed conditions (kind of soll cover that bear a relationshlp to hydrology, and rainfall. Stage-discharge relation is affected by rate of change in stage, and peak stage occurred from 5 to 30 minutes after peak discharge. Only annual (calendar year) peaks are shown.

Peak stages and discharges

\begin{tabular}{|c|c|c|c|c|c|c|c|}
\hline $\begin{array}{l}\text { Calen- } \\
\text { dar } \\
\text { year }\end{array}$ & Date & $\begin{array}{c}\text { Qage } \\
\text { he1ght } \\
\text { (feet) }\end{array}$ & $\begin{array}{c}\text { D1scharge } \\
\text { (cfs) }\end{array}$ & $\begin{array}{l}\text { Calen- } \\
\text { dar } \\
\text { year }\end{array}$ & Date & $\begin{array}{c}\text { Qage } \\
\text { he1ght } \\
\text { (feet) }\end{array}$ & $\begin{array}{c}\text { D1scharge } \\
\text { (cfs) }\end{array}$ \\
\hline $\begin{array}{l}1938 \\
1939 \\
1940\end{array}$ & $\begin{array}{l}\text { Jan. } 23,1938 \\
\text { May } 20,1939 \\
\text { Nov. } 22,1940\end{array}$ & $\begin{array}{r}11.58 \\
5.12 \\
12.90\end{array}$ & $\begin{array}{r}886 \\
295 \\
1,650\end{array}$ & $\begin{array}{l}1941 \\
1942 \\
1943\end{array}$ & $\begin{array}{l}\text { June } 10,1941 \\
\text { Sept. 8, } 1942 \\
\text { June } 5,1943\end{array}$ & $\begin{array}{r}12.63 \\
10.93 \\
4.44\end{array}$ & $\begin{array}{r}1,360 \\
1,000 \\
236 \\
\end{array}$ \\
\hline
\end{tabular}

8-982.51. Brushy Creek watershed SW-18 near R1esel, Tex.(9)

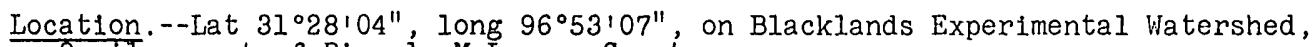
2 miles east of Riesel, McLennan County.

Drainage area. $-0.0048 \mathrm{sq} \mathrm{ml}$.

Gage.--Recording. Datum of gage is $561.0 \mathrm{ft}$ above mean sea level.

Stage-discharge relation.--Defined by theoretical rating for 3-foot H-flume.

Remarks.--Records furnished by U.S. Department of Agriculture, Agricultural Research Service, Blacklands Experimental Watershed at Riesel, Tex. Detailed information is avallable from the Riesel office regarding basin shape and slope, type of solls, erosion condition, watershed conditions (kind of soll cover that bear a relationship to hydrology, and rainfall. Only annual (calendar year) peaks are shown.

Peak stages and discharges

\begin{tabular}{|c|c|c|c|c|c|c|c|}
\hline $\begin{array}{l}\text { Calen- } \\
\text { dar } \\
\text { year }\end{array}$ & Date & $\begin{array}{c}\text { aage } \\
\text { height } \\
\text { (feet) }\end{array}$ & $\begin{array}{c}\text { Discharge } \\
(\text { cfs })\end{array}$ & $\begin{array}{l}\text { Calen- } \\
\text { dar } \\
\text { year }\end{array}$ & Date & $\begin{array}{c}\text { Gage } \\
\text { he1ght } \\
\text { (feet) }\end{array}$ & $\begin{array}{c}\text { Discharge } \\
(\text { cfs })\end{array}$ \\
\hline $\begin{array}{l}1939 \\
1940\end{array}$ & $\begin{array}{lll}\text { May } 20, & 1939 \\
\text { Oct. } & 31, & 1940\end{array}$ & - & $2 \frac{1.6}{3}$ & $\begin{array}{l}1942 \\
1943\end{array}$ & $\begin{array}{ll}\text { Sept. } 8, & 1942 \\
\text { June } & 5,1943\end{array}$ & - & $\begin{array}{r}13 \\
4.2\end{array}$ \\
\hline 1941 & June 10,1941 & - & 15 & $\cdot$ & & & \\
\hline
\end{tabular}

8-982.54. Brushy Creek watershed SW-1l near Riesel, Tex. (9)

Location.--Lat $31^{\circ} 28^{\prime} 02^{\prime \prime}$, long 96 $53^{\prime} 04^{\prime \prime}$, on Blacklands Experimental Watershed, 2 miles east of Riesel, McLennan County.

Drainage area. $--0.0050 \mathrm{sq} \mathrm{ml}$.

Gage.--Recording. Datum of gage is $559.0 \mathrm{ft}$ above mean sea level.

Stage-discharge relation.--Defined by theoretical rating for 3-foot H-flume.

Remarks.--Records furnished by U.S. Department of Agriculture, Agricultural Research Service, Blacklands Experimental Watershed at Riesel, Tex. Deta1led information is avallable from the Riesel office regarding basin shape and slope, type of solls, erosion condition, watershed conditions (kind of soll cover) that bear a relationship to hydrology, and rainfall. Only annual (calendar year) peaks are shown. 
Peak stages and discharges of Brushy Creek watershed SW-11 near R1esel, Tex.

\begin{tabular}{|c|c|c|c|c|c|c|c|}
\hline $\begin{array}{l}\text { Calen- } \\
\text { dar } \\
\text { year }\end{array}$ & Date & $\begin{array}{c}\text { Gage } \\
\text { nelght } \\
\text { (feet) }\end{array}$ & $\begin{array}{c}\text { Discharge } \\
(\mathrm{cfs})\end{array}$ & $\begin{array}{l}\text { Calen- } \\
\text { dar } \\
\text { year }\end{array}$ & Date & $\begin{array}{c}\text { Gage } \\
\text { helght } \\
\text { (feet) }\end{array}$ & $\begin{array}{c}\text { D1scharge } \\
\text { (cfs) }\end{array}$ \\
\hline $\begin{array}{l}1939 \\
1940\end{array}$ & $\begin{array}{lll}\text { May } & 20, & 1939 \\
\text { Oct. } & 31, & 1940\end{array}$ & $\overline{-}$ & $22^{1.8}$ & $\begin{array}{l}1942 \\
1943\end{array}$ & $\begin{array}{l}\text { Sept. } 8,1942 \\
\text { June } 5,1943\end{array}$ & $\overline{-}$ & $\begin{array}{r}12 \\
1.4\end{array}$ \\
\hline 1941. & June 10,1941 & - & 14 & & & & \\
\hline
\end{tabular}

8-982.57. Brushy Creek watershed SW-17 near Riese1, Tex.(9.)

Location.--Lat $31^{\circ} 27^{\prime} 45^{\prime \prime}$, long $96^{\circ} 53^{\prime} 14^{\prime \prime}$, on Blacklands Experimental Watershed, 2 miles east of Riesel, McLennan County.

Drainage area. $--0.0047 \mathrm{sq} \mathrm{ml}$.

Gage.--Recording. Datum of gage is $550.0 \mathrm{ft}$ above mean sea level.

Stage-discharge relation.--Defined by theoretical rating for 3-foot H-flume.

Remarks.--Records furnished by U.S. Department of Agriculture, Agricultural Research Service, Blacklands Experimental Watershed at Riesel, Tex. Deta1led information is available from the Riesel office regarding basin shape and slope, type of soils, erosion condition, watershed conditions (kind of soil cover that bear a relationship to hydrology, and rainfall. Only annual (calendar year) peaks are shown.

\begin{tabular}{|c|c|c|c|c|c|c|c|}
\hline $\begin{array}{l}\text { Calen- } \\
\text { dar } \\
\text { year }\end{array}$ & Date & $\begin{array}{c}\text { Gage } \\
\text { height } \\
\text { (feet) }\end{array}$ & $\begin{array}{l}\text { Discharge } \\
\text { (cfs) }\end{array}$ & $\begin{array}{l}\text { Calen- } \\
\text { dar } \\
\text { year }\end{array}$ & Date & $\begin{array}{c}\text { Gage } \\
\text { nelght } \\
\text { (feet) }\end{array}$ & $\begin{array}{c}\text { Discharge } \\
\text { (cfs) }\end{array}$ \\
\hline $\begin{array}{l}1939 \\
1940\end{array}$ & $\begin{array}{ll}\text { May } 18, & 1939 \\
\text { Oct. } 31, & 1940\end{array}$ & $\overline{-}$ & $21^{2.5}$ & $\begin{array}{l}1952 \\
1953 \\
1954\end{array}$ & $\begin{array}{ll}\text { May } & 23,1952 \\
\text { Mar. } 12,1953 \\
\text { May } 11,1954\end{array}$ & $\overline{-}$ & $\begin{array}{l}1.9 \\
4.6 \\
2.6\end{array}$ \\
\hline $\begin{array}{l}1941 \\
1942 \\
1943\end{array}$ & $\begin{array}{l}\text { June } 10,1941 \\
\text { Sept. } 8,1942 \\
\text { June } 10, \\
1943\end{array}$ & - & $\begin{array}{l}19 \\
18 \\
13\end{array}$ & & Mar. 21, 1955 & & 3.7 \\
\hline 1943 & June 10,1943 & - & 13 & $\begin{array}{l}1956 \\
1957\end{array}$ & $\begin{array}{l}\text { Nov. 4, } 1956 \\
\text { Apr. 19, } 1957\end{array}$ & $\overline{-}$ & $10^{.1}$ \\
\hline $\begin{array}{l}1948 \\
1949 \\
1950\end{array}$ & $\begin{array}{l}\text { Apr. } 25,1948 \\
\text { July } 4,1949 \\
\text { Feb. } 12,1950\end{array}$ & $\overline{-}$ & $\begin{array}{l}11 \\
3.8 \\
11\end{array}$ & $\begin{array}{l}1958 \\
1959 \\
1960\end{array}$ & $\begin{array}{l}\text { Aug. } 24,1958 \\
\text { June } 23,1959 \\
\text { Jan. } 13,1960\end{array}$ & $\overline{-}$ & $\begin{array}{l}.6 \\
6.5 \\
1.2\end{array}$ \\
\hline 1951 & June 16,1951 & $=$ & .5 & $\begin{array}{l}1961 \\
1962 \\
1963\end{array}$ & $\begin{array}{l}\text { June } 25,1961 \\
\text { June } 9,1962 \\
\end{array}$ & $\begin{array}{l}- \\
-\end{array}$ & $\begin{array}{c}1.8 \\
12 \\
0\end{array}$ \\
\hline
\end{tabular}

\section{8-982.6. Brushy Creek watershed SW-5 near Riese1, Tex.(9)}

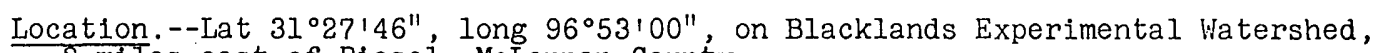
2 miles east of Riesel, McLennan County.

Drainage area. $--0.0048 \mathrm{sq} \mathrm{mi}$.

Gage.--Recording... Datum of gage is 546:6 ft above mean sea level.

Stage-discharge relation.--Defined by theoretical rating for 3-foot $\mathrm{H}$-flume.

Remarks.--Records furnished by U.S. Department of Agriculture, Agricultural Research Service, Blacklands Experimental Watershed at Riesel, Tex. Deta1led information is available from the Riesel office regarding basin shape and slope, type of solls, erosion condition, watershed conditions (kind of soil cover that bear a relationship to hydrology, and rainfall. Only annual (calendar year) peaks are shown.

Peak stages and discharges

\begin{tabular}{|c|c|c|c|c|c|c|c|}
\hline $\begin{array}{l}\text { Calen- } \\
\text { dar } \\
\text { year }\end{array}$ & Date & $\begin{array}{c}\text { Gage } \\
\text { he1ght } \\
\text { (feet) }\end{array}$ & $\begin{array}{c}\text { D1scharge } \\
(\mathrm{cfs})\end{array}$ & $\begin{array}{l}\text { Calen- } \\
\text { dar } \\
\text { year }\end{array}$ & Date & $\begin{array}{c}\text { Gage } \\
\text { nelght } \\
\text { (feet) }\end{array}$ & $\begin{array}{c}\text { D1scharge } \\
(\text { cfs })\end{array}$ \\
\hline $\begin{array}{l}1939 \\
1940\end{array}$ & 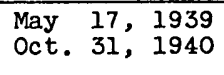 & $\overline{-}$ & $20^{2.3}$ & $\begin{array}{l}1942 \\
1943\end{array}$ & $\begin{array}{ll}\text { Dec. } & 26,1942 \\
\text { June } & 5,1943\end{array}$ & - & $\begin{array}{l}14 \\
9.6\end{array}$ \\
\hline 1941 & June 10,1941 & - & 19 & & & & \\
\hline
\end{tabular}


8-982.63. Brushy Creek watershed $W-1$ near Riesel, Tex.(9)

Jocation.--Lat $31^{\circ} 27^{\prime} 27^{\prime \prime}$, long $96^{\circ} 52^{\prime} 48^{\prime \prime}$, on Blacklands Experimental Watershed, $2.2 \mathrm{~m} i l e s$ southeast of Riesel, McLonnan County.

Drainage area. $--0.275 \mathrm{sq} \mathrm{ml}$.

Gage.--Recording. Datum of gage is $520.4 \mathrm{ft}$ above mean sea level.

Stage-discharge relation.--Defined by theoretical rating for 15-foot Parshall flume, modifled with weir for measuring low flows.

Remarks.--Records furnished by U.S. Department of Agriculture, Agricultural Research Service, Blacklands Experimental Watershed at Riesel, Tex. Detailed information is available from the Riesel office regarding basin shape and slope, type of solls, erosion conditions, watershed conditions (kind of soll cover that bear a relationship to hydrology, and rainfall. The drainage area is artificially controlled, therefore no gage heights are shown. Only annual (calendar year) peaks are shown.

Peak stages and discharges

\begin{tabular}{|c|c|c|c|c|c|c|c|}
\hline $\begin{array}{l}\text { Calen- } \\
\text { dar } \\
\text { year }\end{array}$ & Date & $\begin{array}{c}\text { Gage } \\
\text { height } \\
\text { (feet) }\end{array}$ & $\begin{array}{c}\text { Discharge } \\
\text { (cfs) }\end{array}$ & \begin{tabular}{|l} 
Calen- \\
dar \\
year
\end{tabular} & Date & $\begin{array}{c}\text { Gage } \\
\text { nelght } \\
\text { (feet) }\end{array}$ & $\begin{array}{c}\text { Discharge } \\
(\text { cfs) }\end{array}$ \\
\hline $\begin{array}{l}1938 \\
1939\end{array}$ & $\begin{array}{l}\text { Feb. } 16,1938 \\
\text { May } 20,1939\end{array}$ & - & $\begin{array}{r}106 \\
67\end{array}$ & 1950 & Feb. 12,1950 & - & 334 \\
\hline 1940 & Nov. 22,1940 & - & 472 & $\begin{array}{l}1951 \\
1952\end{array}$ & $\begin{array}{ll}\text { June } & 16,1951 \\
\text { May } 23, & 1952\end{array}$ & - & $\begin{array}{l}23 \\
94\end{array}$ \\
\hline 1941 & June 10,1941 & - & 603 & 1953 & May 12,1953 & - & 211 \\
\hline 1942 & Sept. 8, 1942 & - & 476 & 1954 & 11,1954 & - & 236 \\
\hline $\begin{array}{l}1943 \\
1944\end{array}$ & $\begin{array}{lll}\text { June } & 5,1943 \\
\text { May } & 1,1944\end{array}$ & $\overline{-}$ & $\begin{array}{l}192 \\
800\end{array}$ & 1955 & Mar. 21, 1955 & - & 268 \\
\hline 1945 & Mar. 3,1945 & - & 319 & 1956 & 4, 1956 & - & 44 \\
\hline \multirow{5}{*}{$\begin{array}{l}1946 \\
1947 \\
1948 \\
1949\end{array}$} & \multirow{5}{*}{$\begin{array}{lrr}\text { May } & 12, & 1946 \\
\text { May } 20, & 1947 \\
\text { Apr. } & 25,1948 \\
\text { July } & 4, & 1949\end{array}$} & \multirow{5}{*}{$\begin{array}{l}- \\
- \\
-\end{array}$} & \multirow{5}{*}{$\begin{array}{r}382 \\
78 \\
287 \\
245\end{array}$} & 1958 & Aug. 24,1958 & - & 55 \\
\hline & & & & 1959 & June $23,1.959$ & - & 335 \\
\hline & & & & 1960 & Oct. 18,1960 & - & 35 \\
\hline & & & & -1961 & June 25,196 & - & 80 \\
\hline & & & & $\begin{array}{l}1962 \\
1963 \\
\end{array}$ & June 9, 1962 & - & $\begin{array}{r}387 \\
0 \\
\end{array}$ \\
\hline
\end{tabular}

8-982.66. Brushy Creek watershed SW-3 near Riesel, Tex.(9)

Location.--Lat $31^{\circ} 27129^{\prime \prime}$, long $96^{\circ} 53^{\prime} 12^{\prime \prime}$, on Blacklands Experimental Watershed, 2 miles east of Riesel, McLennan County.

Drainage area. $--0.0048 \mathrm{sq} \mathrm{mi}$.

Gage.--Recording. Datum of gage is $553.8 \mathrm{ft}$ above mean sea level.

Stage-discharge relation.--Defined by theoretical rating for 3-foot $\mathrm{H}$-flume.

Remarks.--Records furnished by U.S. Department of Agriculture, Agricultural Research Service, Blacklands Experimental Watershed at Riesel, Tex. Detailed information is avallable from the Riesel office regarding basin shape and slope, type of soils, erosion condition, watershed conditions (kind of soil cover that bear a relationship to hydrology, and rainfall. Only annual (çalendar" year) peaks are shown.

Peak stages and discharges

\begin{tabular}{|c|c|c|c|c|c|c|c|}
\hline $\begin{array}{l}\text { Calen- } \\
\text { dar } \\
\text { year }\end{array}$ & Date & $\begin{array}{c}\text { Qage } \\
\text { height } \\
\text { (feet) }\end{array}$ & $\begin{array}{c}\text { D1scharge } \\
\text { (cfs) }\end{array}$ & $\begin{array}{l}\text { Calen- } \\
\text { dar } \\
\text { year }\end{array}$ & Date & $\begin{array}{c}\text { Gage } \\
\text { helght } \\
\text { (feet) }\end{array}$ & $\begin{array}{c}\text { Discharge } \\
(\mathrm{cfs})\end{array}$ \\
\hline $\begin{array}{l}1939 \\
1940\end{array}$ & $\begin{array}{ll}\text { May } 20, & 1939 \\
\text { Nov. } & 22,1940\end{array}$ & - & $10^{1.4}$ & $\begin{array}{l}1942 \\
1943\end{array}$ & $\begin{array}{l}\text { Sept. } 8,1942 \\
\text { June } 5,1943\end{array}$ & - & $\begin{array}{l}9.2 \\
8.9\end{array}$ \\
\hline 1941 & June 10,1941 & - & 15 & & & & \\
\hline
\end{tabular}


8-982.69. Brushy Creek watershed $W-6$ near Riesel, Tex.(9)

Location.--Lat $31^{\circ} 27^{\prime} 24^{\prime \prime}$, long $96^{\circ} 53^{\prime} 11^{\prime \prime}$, on Blacklands Experimental Watershed, 2.5 miles southeast of Riesel, McLennan County.

Drainage area. $--0.0661 \mathrm{sq} \mathrm{ml}$.

Gage.--Recording. Datum of gage is $538 \mathrm{ft}$ above mean sea level.

Stage-discharge relation.--Defined by theoretical rating for 6-foot Parshall f'Jume, modified with weir for measuring low flows.

Remarks.--Records furnished by U.S. Department of Agriculture, Agricultural Research Service, Blacklands Experimental Watershed at Riesel, Tex. Detailed information is available from the Riesel office regarding basin shape and slope, type of soils, erosion condition, watershed conditions (kind of soil cover that bear a relationship to hydrology, and rainfall. Only annual (calendar year) peaks are shown.

Peak stages and discharges

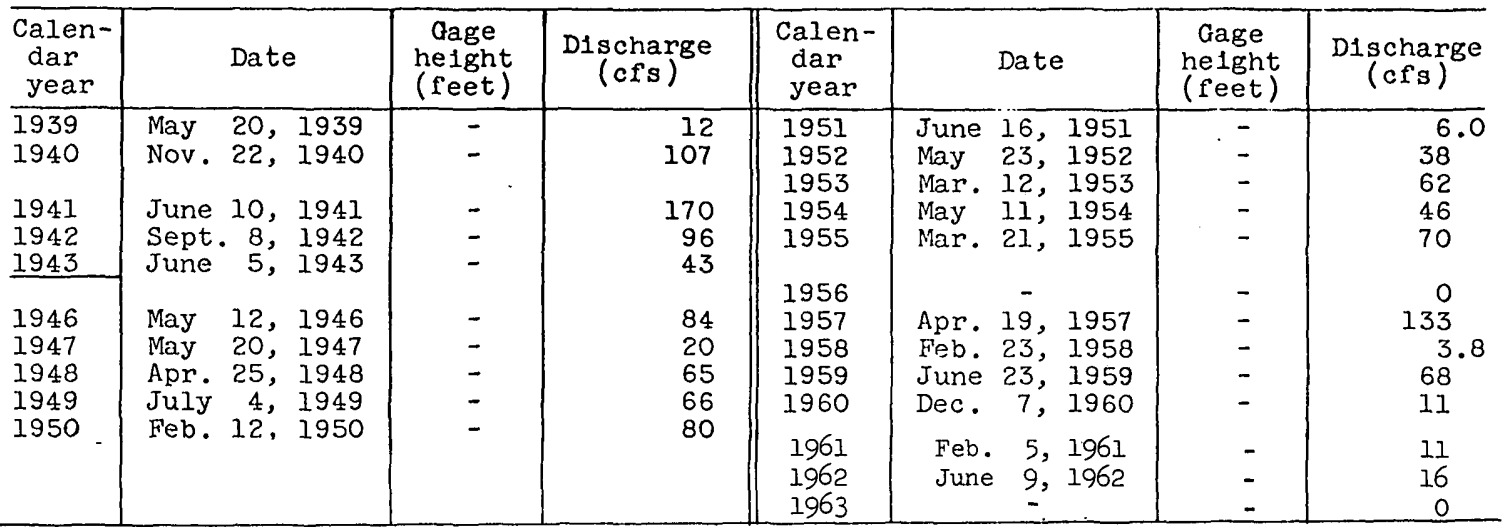

8-982.72. Brushy Creek watershed SW-2 near Riesel, Tex.(9)

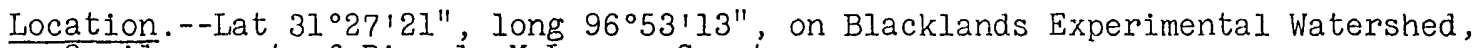
2 miles east of Riese1, McLennan County.

Drainage area. $-0.0042 \mathrm{sq} \mathrm{mi}$.

Gage.--Recording. Datum of gage is $544.0 \mathrm{ft}$ above mean sea level.

Stage-discharge relation.--Defined by theoretical rating for 3-foot H-flume.

Remarks.--Records furnished by U.S. Department of Agriculture, Agricultural Research Service, Blacklands Experimental Watershed at Riesel, Tex. Detailed information is available from the Riesel office regarding basin shape and slope, type of soils, erosion condition, watershed conditions (kind of soil cover) that bear a relationship to hydrology, and rainfall. Only annual (calendar year) peaks are shown.

Peak stages and discharges

\begin{tabular}{|c|c|c|c|c|c|c|c|c|}
\hline $\begin{array}{l}\text { Calen- } \\
\text { dar } \\
\text { year }\end{array}$ & & Date & $\begin{array}{c}\text { Gage } \\
\text { height } \\
\text { (feet) }\end{array}$ & $\begin{array}{c}\text { Discharge } \\
(\text { cfs })\end{array}$ & $\begin{array}{l}\text { Calen- } \\
\text { dar } \\
\text { year }\end{array}$ & Date & $\begin{array}{c}\text { Gage } \\
\text { helght } \\
\text { (feet) }\end{array}$ & $\begin{array}{c}\text { Discharge } \\
(\mathrm{cfs})\end{array}$ \\
\hline $\begin{array}{l}1939 \\
1940\end{array}$ & $\begin{array}{l}\text { May } \\
\text { Oct. }\end{array}$ & $\begin{array}{l}20,1939 \\
31,1940\end{array}$ & - & $\begin{array}{l}1.3 \\
8.0\end{array}$ & $\begin{array}{l}1942 \\
1943\end{array}$ & $\begin{array}{l}\text { Sept. } 8,1942 \\
\text { June } 5,1943\end{array}$ & - & $\begin{array}{l}12 \\
12\end{array}$ \\
\hline 1941 & June & $10, \quad 1941$ & - & 15 & & & & \\
\hline
\end{tabular}


Location.--Lat $31^{\circ} 27^{\prime} 12^{\prime \prime}$, long 96 ${ }^{\circ} 53^{\prime} 00^{\prime \prime}$, on Blacklands Experimental Watershed, 2.8 miles southeast of Riesel, McLennan County.

Drainage area. $-0.0308 \mathrm{sq} \mathrm{ml}$.

Gage.--Recording. Datum of gage is $540 \mathrm{ft}$ above mean sea level.

Stage-discharge relation.--Defined by theoretical rating for 6-foot Parshall flume, modifled with weir for measuring low flows.

Remarks.--Records furnished by U.S. Department of Agriculture, Agricultural Research Service, Blacklands Experimental Watershed at Rlesel, Tex. Detailed information is avallable from the Riesel office regarding basin shape and slope, type of solls, erosion condition, watershed conditions (kind of soll cover that bear a relationship to hydrology, and rainfall. Only annual (calendar year) peaks are shown.

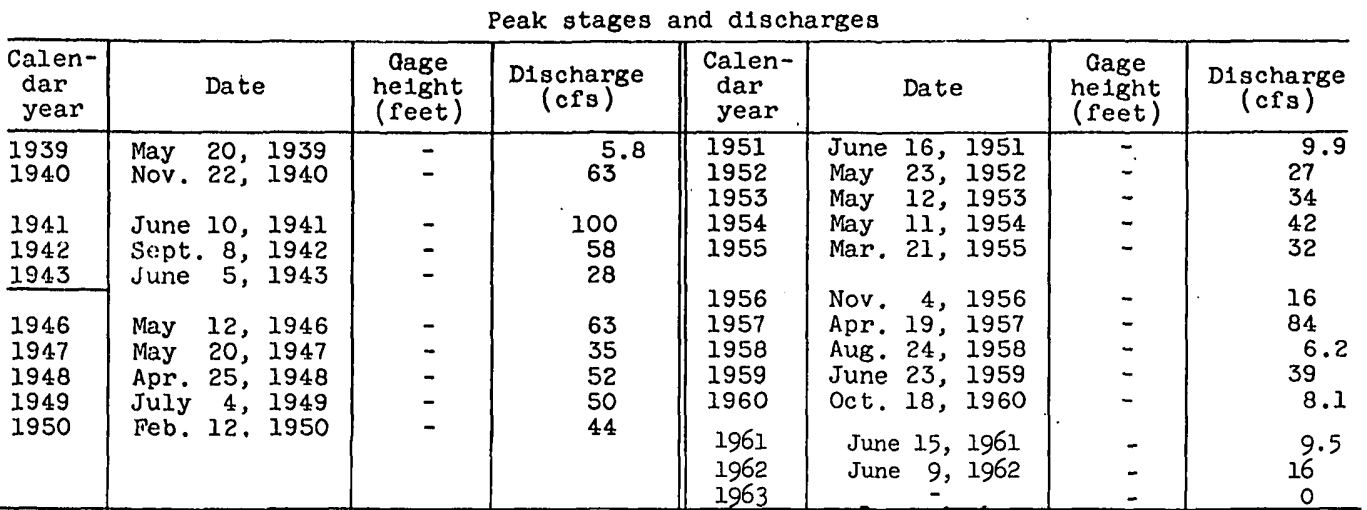

8-982.81. Brushy Creek watershed W-2 near R1esel, Tex. (9)

Location.--Lat $31^{\circ} 27^{\prime} 19^{\prime \prime}$, long 96 $52^{\prime} 55^{\prime \prime}$, on Blacklands Experimental Watershed, 2.2 miles southeast of Riesel, McLennan County.

Dralnage area. $--0.203 \mathrm{sq} \mathrm{ml}$.

Gage.--Recording. Datum of gage 1s $521.2 \mathrm{ft}$ above mean sea level.

Stage-discharge relation.--Defined by theoretical rating for 15-foot Parshall flume, modified with weir for measuring low flows.

Remarks.--Records furnished by U.S. Department of Agriculture, Agricultural Research Service, Blacklands Experimental Watershed at Riesel, Tex. Detailed information is avallable from the Rlesel office regarding basin shape and slope, type of solls, erosion condition, watershed conditions ( $k$ ind of soli cover that bear a relationship to hydrology, and rainfall. Only annual (calendar year) peaks are shown.

Peak stages and discharges

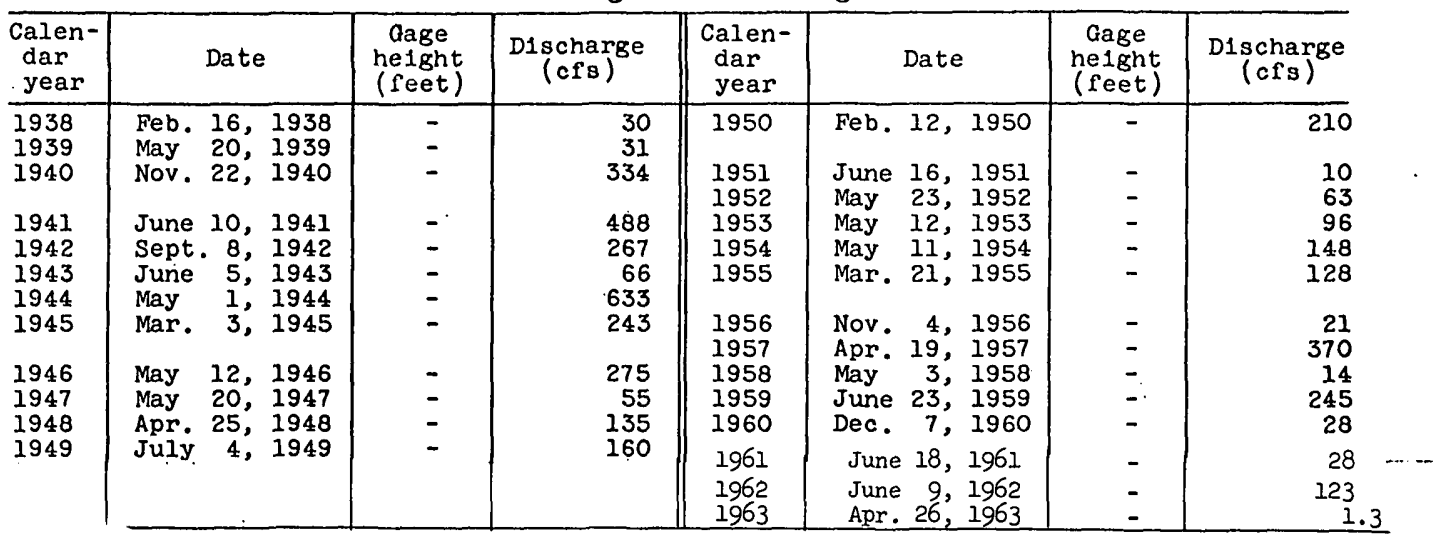


8-982.84. Brushy. Creek watershed SW-6 near Rlesel, Tex.(9)

Location.--Lat $31^{\circ} 27 ! 13^{\prime \prime}$, long $96^{\circ} 52^{\prime 4} 7^{\prime \prime}$, on Blacklands Experimental Watershed, 2 miles east of Riesel, McLennan County.

Drainage area. $--0.0048 \mathrm{sq} \mathrm{ml}$.

Gage.--Recording. Datum of gage is $525.7 \mathrm{ft}$ above mean sea level.

Stage-discharge relation.--Defined by theoretical rating for 3-foot $\mathrm{H}$-flume:

Remarks.--Records furnished by U.S. Department of Agriculture, Agricultural

Research Service, Blacklands Experimental Watershed at Riesel, Tex. Detailed

information is available from the Riesel office regarding basin shape and

slope, type of soils, erosion condition, watershed conditions (kind of soil

cover) that bear a relationship to hydrology, and rainfall. Only annual

(calendar year) peaks are shown.

\begin{tabular}{|c|c|c|c|c|c|c|c|}
\hline $\begin{array}{l}\text { Calen- } \\
\text { dar } \\
\text { year }\end{array}$ & Date & $\begin{array}{c}\text { Gage } \\
\text { nelght } \\
\text { (feet) }\end{array}$ & $\begin{array}{c}\text { Discharge } \\
\text { (cfs) }\end{array}$ & $\begin{array}{l}\text { Calen- } \\
\text { dar } \\
\text { year }\end{array}$ & Date & $\begin{array}{c}\text { Gage } \\
\text { nelght } \\
\text { (feet) }\end{array}$ & $\begin{array}{c}\text { Discharge } \\
\text { (cfs) }\end{array}$ \\
\hline $\begin{array}{l}1939 \\
1940\end{array}$ & $\begin{array}{lll}\text { May } & 18, & 1939 \\
\text { Oct. } & 31, & 1940\end{array}$ & - & $\begin{array}{l}6.9 \\
16\end{array}$ & $\begin{array}{l}1942 \\
1943\end{array}$ & $\begin{array}{lr}\text { Dec. } 26, & 1942 \\
\text { June } 5, & 1943\end{array}$ & $\bar{z}$ & $\frac{14}{4.0}$ \\
\hline 1941 & June 10,1941 & - & 18 & & & & \\
\hline
\end{tabular}

8-1070. Big Elm Creek near Temple, Tex. (9)

Location.--Lat $31^{\circ} \mathrm{O} 3^{\prime}$, long $97^{\circ} 15^{\prime}$, $350 \mathrm{ft}$ downstream from mouth of Cottonwood Creek, about $0.6 \mathrm{mile}$ upstream from Little Elm Creek, and 6 miles east of Temple, Bell County.

Drainage area.--68.5 sq mi.

Gage.--Recording. Prior to May 11, 1934, staff gage at site $60 \mathrm{ft}$ downstream at datum $0.35 \mathrm{ft}$ lower. Historical data.--Flood of September 1921 reached a stage of about $21 \mathrm{ft}$, from information by local residents.

Remarks.--Only maximum annual peaks are shown.

\begin{tabular}{c|c|c|c|c|c|c|c}
\hline $\begin{array}{c}\text { Water } \\
\text { year }\end{array}$ & Date & $\begin{array}{c}\text { Ga.ge } \\
\text { height } \\
\text { (feet) }\end{array}$ & $\begin{array}{c}\text { Discharge } \\
\text { (cfs) }\end{array}$ & $\begin{array}{c}\text { Water } \\
\text { year }\end{array}$ & Da.te & $\begin{array}{c}\text { Gage } \\
\text { height } \\
\text { (feet) }\end{array}$ & $\begin{array}{c}\text { Discharge } \\
\text { (cfs) }\end{array}$ \\
\hline 1934 & Apr. 6, 1934 & $\begin{array}{l}16.15 \\
18.05\end{array}$ & $\begin{array}{r}6,130 \\
15,700\end{array}$ & 1936 & June 30, 1936 & 17.82 & 14,200 \\
\hline
\end{tabular}

8-1080. North Elm Creek near Ben Arnold, Tex. (17)

Location.--Lat $30^{\circ} 57^{\prime}$, long $97^{\circ} 03^{\prime}$, at bridge on county road between Ben Arnold and Yarrellton, $3 \frac{1}{2}$ miles west of Ben Arnold, Milam County.

Drainage area. $--30.3 \mathrm{sq} \mathrm{mi}$.

Gage.--Recording.

Stage-discharge relation.--Defined by current-meter measurements below $800 \mathrm{cfs}$, and extended above on basis of indirect measurement of peak discharge.

Remarks:--Only annual peaks are shown.

\begin{tabular}{c|c|c|c||c|c|c|c|}
\hline $\begin{array}{c}\text { Water } \\
\text { year }\end{array}$ & Date & $\begin{array}{c}\text { Ga.ge } \\
\text { height } \\
\text { (feet) }\end{array}$ & $\begin{array}{c}\text { Discharge } \\
\text { (cfs) }\end{array}$ & $\begin{array}{c}\text { Water } \\
\text { year }\end{array}$ & Date & $\begin{array}{c}\text { Gage } \\
\text { height } \\
\text { (feet) }\end{array}$ & $\begin{array}{c}\text { Discharge } \\
\text { (cfs) }\end{array}$ \\
\hline 1935 & June 15, 1935 & 6.92 & 2,040 & 1936 & Dec. 6, 1935 & 8.90 & 3,540 \\
\hline
\end{tabular}


BRAZOS RIVER BASIN

8-1150. Big Creek near Needville, Tex. (12)

Location.--Lat $29^{\circ} 28^{\prime} 35^{\prime \prime}$, long $95^{\circ} 48^{\prime} 45^{\prime \prime}$, at bridge on State Highway $36,1.5$ miles downstream from

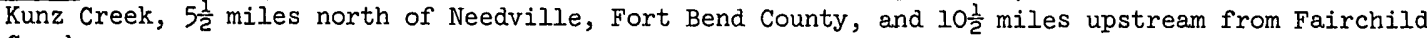
Creek.

Drainage area.-- $-37.6 \mathrm{sq} \mathrm{mi}$.

Gase.--Nonrecording prior to Mar. 15, 1952, and May 29, 1959, to Mar. 29, 1960. Recording from Mar. 15, 1952, to May 28, 1959, and after Mar. 29, 1960. Datum of ga.ge is $69.39 \mathrm{ft}$ above mean sea level, datum of 1929, Houston supplementary adjustment of 1943.

Stage-discharge relation.--Defined by current-meter measurements.

Bankfull stage.--lo ft.

Historical data.--Maximum stage since 1913 is that of August 1945, from information by local resident.

Remarks.--Channel was rectified in April 1955, thereby greatly increasing its capacity. Base for partial-duration series, $500 \mathrm{cfs}$.

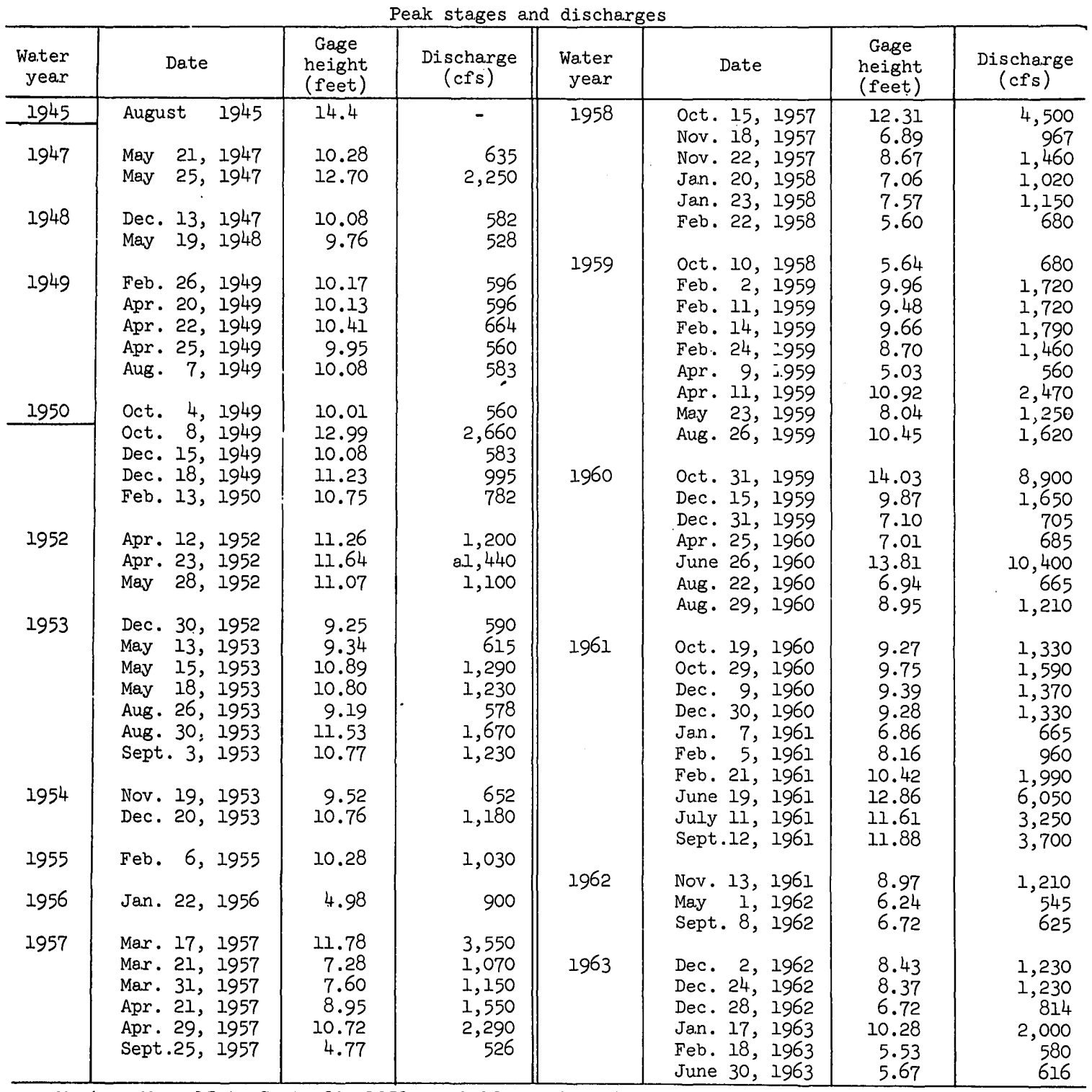

a. Maximum Mar. 15 to Sept. 30, 1952; probably maximum for year. 
8-1155. Fairchild Creek near Needville, Tex.(12)

Location. - -Lat $29^{\circ} 26^{\prime} 45^{\prime \prime}$, long $95^{\circ} 45^{\prime} 40^{\prime \prime}$, at downstream side of county road bridge, 3.0 miles upstream from mouth, and $5 \frac{1}{2}$ miles northeast of Needville, Fort Bend County.

Drainage area. $--24.9 \mathrm{sq} \mathrm{mi}$.

Gage.--Nonrecording. Datum of gage is $60.42 \mathrm{ft}$ above mean sea level, datum of I929, Houston supplementary adjustment of 1943.

Stage-discharge relation.--Defined by current-meter measurements below $1,800 \mathrm{cfs}$.

H1storical data.--Flood of 0ct. 31, 1959, is highest since 1910, from information by local residents.

Remarks.--Base for partial-duration series, $500 \mathrm{cfs}$.

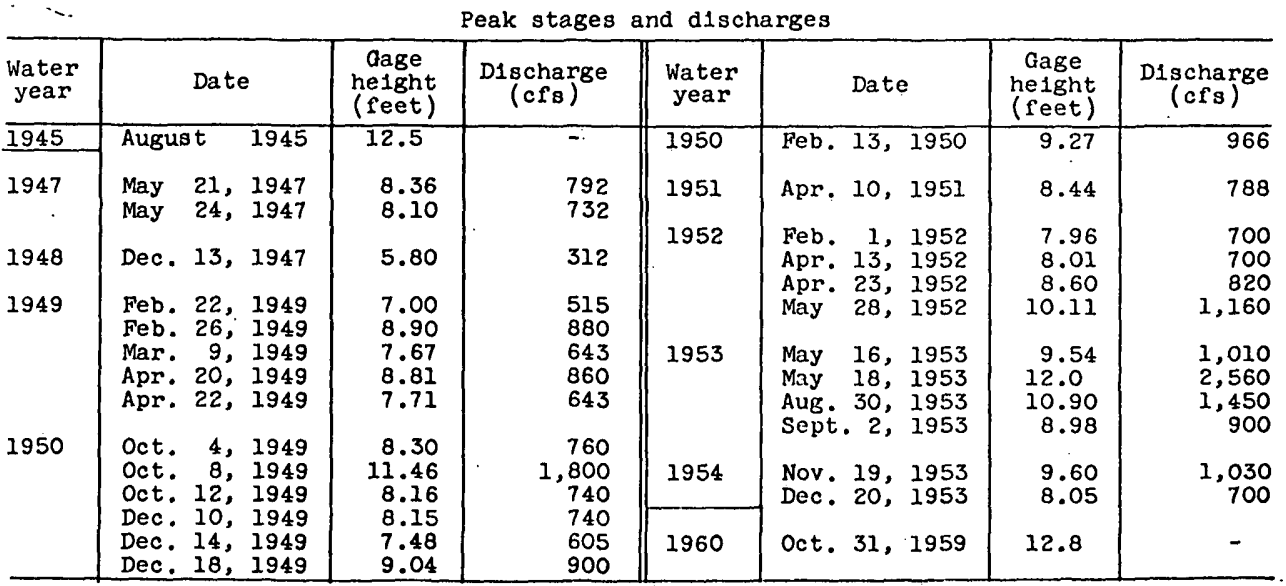

8-1164. Dry Creek near Rosenberg, Tex. (12)

Location.--Lat $29^{\circ} 30^{\prime} 42^{\prime \prime}$, long $95^{\circ} 44^{\prime} 45^{\prime \prime}$, on right bank, 38 ft downstream from county road bridge, $8.2 \mathrm{miles}$ upstream from Smithers Lake spillway, and 5.0 miles southeast of Rosenberg, Fort Bend County.

Drainage area. $--8.53 \mathrm{sq} \mathrm{mi}$

Gage.--Recording. Datum of gage is $71.90 \mathrm{ft}$ above mean sea level, datum of 1929, supplementary adjustment of 1943.

Stage-discharge relation.--Defined by current-meter measurements.

Bankfull stage..--10 ft.

Historical data.--Highest flood since at least 1932, Oct. 31, 1959, from information by local residents.

Remarks.--Base for partial-duration series, $250 \mathrm{cfs}$.

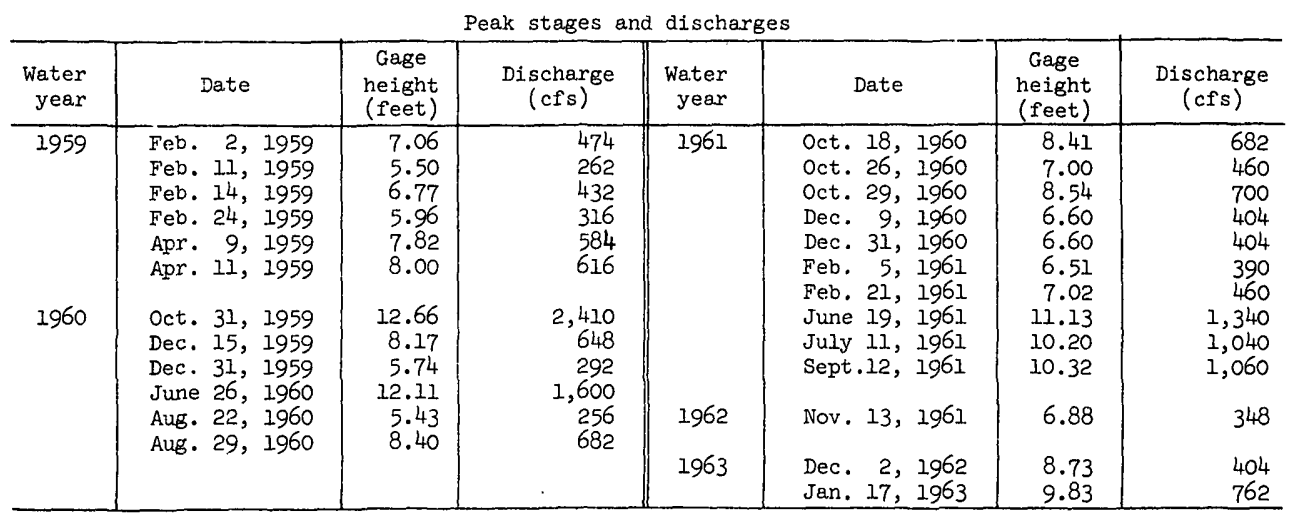


8-1165. Dry Creek near Richmond, Tex. (12)

Location.--Lat $29^{\circ} 30^{\prime} 19^{\prime \prime}$, long $95^{\circ} 42^{\prime} 39^{\prime \prime}$, at downstream side of bridge on county road, 2.0 miles upstream from Farm Road $762,2.3$ miles south of Gulf, Colorado and Santa Fe Ra1lway Co. bridge at Crabb, 6 miles upstream from Smither's Lake (Lake George) splilway, and 6.0 miles southeast of Richmond, Fort Bend County.

Drainage area. $--11.4 \mathrm{sq} \mathrm{ml}$.

Gage.--Nonrecording prior to June 30, 1950; recording thereafter. Datum of gage Is $64.5 \mathrm{ft}$ above mean sea level, datum of 1.929, Houston supplementary adjustment of 1943. At datum $1.50 \mathrm{ft}$ higher prior to June 30, 1950.

Stage-discharge relation.--Defined by current-meter measurements below $1,200 \mathrm{cfs}$ and extended above on basis of area-velocity study and logarithmic plotting.

Remarks.--Channel rectifled in 1952 and in 1956. It has been estimated that channel changes would affect the stage of floods of the magnitude of those in 1945 and in 1953 by as much as 2 or 3 ft. At site 2.2 miles upstream (Dry Creek near Rosenberg), less affected by channel rectification, the highest flood since at least 1932 was that of Oct. 31, 1959; the next two in order of magnitude are those of 1945 and 1953 from information by local residents. Base for partial-duration series, $200 \mathrm{cfs}$.

Peak stages and discharges

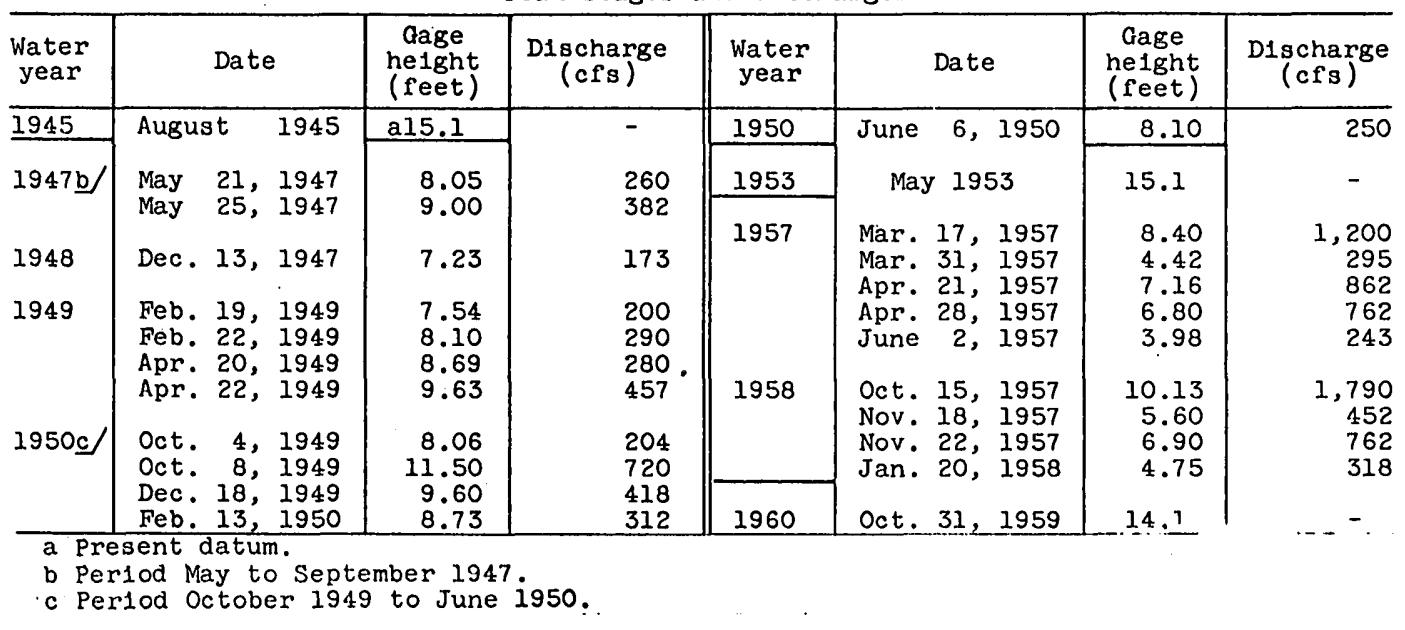

COLORADO RIVER . BASIN

8-1190. Bluff Creek near Ira, Tex. (8)

Location.--Lat $32^{\circ} 35^{\prime} 29^{\prime \prime}$, long $101^{\circ} 03^{\prime} 05^{\prime \prime}$, near left bank on downstream side of pier of abandoned county road bridge, $426 \mathrm{ft}$ downstream from bridge on Farm to Market Road 1606, 1.8 miles upstrecm from mouth, 2.8 miles west of Ira, Scurry County, and 11.6 miles southwest of Snyder.

Dralnage area. $--42.6 \mathrm{sq} \mathrm{ml}$.

Gage.--Recording. Datum of gage is $2,177.95 \mathrm{ft}$ above mean sea level, datum of 1929 .

Stage-discharge relation.--Defined by current-meter measurements below 1,600 $\mathrm{cf}$ 's and by slope-area measurements at 2,380 and $5,200 \mathrm{cfs}$.

Bankfull stage. $--7 \mathrm{ft}$.

Historlcal data.--Maximum stage since at least 1906 occurred in 1939, stage unknown. Flood in 1948 is the second highest since 1906.

Remarks.--Base for partial-duration series, $250 \mathrm{cfs}$. 
COLORADO RIVER BASIN

Peak stages and d1scharges of Bluff Creek near Ira, Tex.

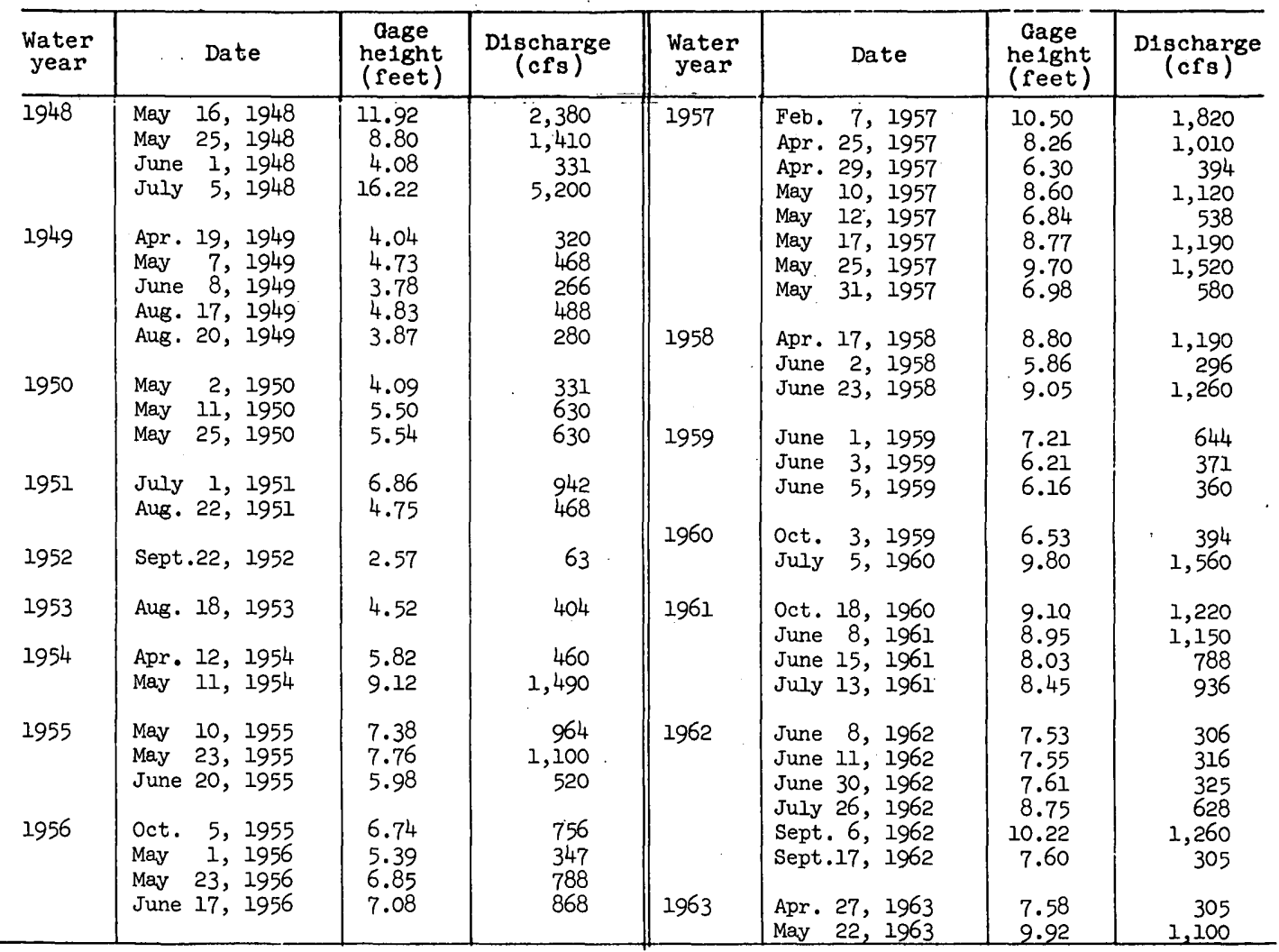

8-1220. Graze Creek near Westbrook, Tex.(8)

Location:--Lat $32^{\circ} 25^{\prime} 03^{\prime \prime}$, long $101^{\circ} 01^{\prime} 10^{\prime \prime}, 1.2$ miles upstream from mouth and $4.2 \mathrm{miles}$ north of Westbrook, Mitchell County.

Drainage area. $-21.1 \mathrm{sq} \mathrm{mi}$.

Gage --Recording. Datum of gage is $2,092.66 \mathrm{ft}$ above mean sea level, datum of 1929.

Stage-discharge relation.--Defined by current-meter measurements below $600 \mathrm{cfs}$ and by slope-area measurement at $1,630 \mathrm{cfs}$.

Historical data.--Maximum stage since at least 1919, that of June 1939, from information by local resident.

Remarks.--Base for partial-duration series, $50 \mathrm{cfs}$.

Peak stages and discharges

\begin{tabular}{|c|c|c|c|c|c|c|c|}
\hline $\begin{array}{l}\text { Water } \\
\text { year }\end{array}$ & Date & $\begin{array}{l}\text { Gage } \\
\text { nelght } \\
\text { (feet) }\end{array}$ & $\begin{array}{c}\text { Discharge } \\
\text { (cfs) }\end{array}$ & $\begin{array}{l}\text { Water } \\
\text { year }\end{array}$ & Date & $\begin{array}{c}\text { Gage } \\
\text { he1ght } \\
\text { (feet) }\end{array}$ & $\begin{array}{l}\text { Discharge } \\
\text { (cfs) }\end{array}$ \\
\hline 1939 & June 1939 & 19.0 & - & 1957 & 9,1957 & 1.66 & 70 \\
\hline 1954 & June 29, 1954 & 2.18 & 125 & & May 11,1957 & $\begin{array}{r}2.27 \\
12.77\end{array}$ & $\begin{array}{r}130 \\
1,800\end{array}$ \\
\hline 1955 & $\begin{array}{lll}\text { May } 11, & 1955 \\
\text { May } 23, & 1955 \\
\text { June } 16,1955\end{array}$ & $\begin{array}{l}3.61 \\
2.70 \\
1.60\end{array}$ & $\begin{array}{r}251 \\
170 \\
62\end{array}$ & & $\begin{array}{lll}\text { May } & 18, & 1957 \\
\text { May } & 25, & 1957 \\
\text { May } & 31, & 1957\end{array}$ & $\begin{array}{l}4.88 \\
8.04 \\
3.28\end{array}$ & $\begin{array}{l}372 \\
750 \\
224\end{array}$ \\
\hline & Aug. 21, 1955 & 2.03 & 110 & 1958 & $\begin{array}{l}\text { Nov. } 5,1957 \\
\text { June } 23,1958\end{array}$ & $\begin{array}{l}1.55 \\
2.55\end{array}$ & $\begin{array}{r}54 \\
156\end{array}$ \\
\hline 1956 & $\begin{array}{ll}\text { Oct. } & 1,1955 \\
\text { May } & 1, \\
1956\end{array}$ & $\begin{array}{r}1.82 \\
12.23\end{array}$ & $\begin{array}{r}88 \\
1,620\end{array}$ & & Sept.27, 1958 & 1.92 & 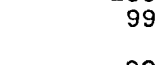 \\
\hline 1957 & $\begin{array}{l}\text { Oct. } 29,1956 \\
\text { Apr. } 25,1957 \\
\text { Apr. } 29,1957\end{array}$ & $\begin{array}{r}1.77 \\
12.38 \\
9.90 \\
\end{array}$ & $\begin{array}{r}83 \\
1,680 \\
1,080\end{array}$ & 1959 & $\begin{array}{lll}\text { May } 1, & 1959 \\
\text { July 1, } 1959 \\
\text { Sept.10, } & 1959\end{array}$ & $\begin{array}{l}1.75 \\
2.00 \\
1.57\end{array}$ & $\begin{array}{r}80 \\
107 \\
58\end{array}$ \\
\hline
\end{tabular}


Location.--Lat $31^{\circ} 19^{\prime} 03^{\prime \prime}$, long $100^{\circ} 27^{\prime} 30^{\prime \prime}$, on left bank, $210 \mathrm{ft}$ upstream from county road, $0.9 \mathrm{mile}$ west of U. S. Highway 277, 2.4 miles upstream from mouth, and 10.1 miles south of San Angelo, Tom Green County.

Drainage area.--84.9 sq mi.

Gage.--Recording. Datum of gage is 1,910.51 ft above mean sea level, datum of 1929.

Stage-discharge relation.---Defined by current-meter measurements below 2,100 cfs, and extended above on basis of slope-area measurements of $30,500 \mathrm{cfs}$.

Bankfull stage.--10 ft.

Historical data.--Maximum stage since at least 1908, $14.36 \mathrm{ft}$ Sept. 15, 1936 (discharge, 30,500 cfs, by slope-area measurement of peak discharge).

Remarks:--Base for partial-duration series, 100 cfs.

Peak stages and discharges

\begin{tabular}{|c|c|c|c|c|c|c|c|}
\hline $\begin{array}{r}\text { Water } \\
\text { year }\end{array}$ & Date & $\begin{array}{c}\text { Gage } \\
\text { height } \\
\text { (feet) }\end{array}$ & $\begin{array}{l}\text { Discharge } \\
\text { (cfs) }\end{array}$ & $\begin{array}{l}\text { Water } \\
\text { year }\end{array}$ & Date & $\begin{array}{c}\text { Gage } \\
\text { height } \\
\text { (feet) }\end{array}$ & $\begin{array}{c}\text { Discharge } \\
\text { (cfs) }\end{array}$ \\
\hline $\begin{array}{l}1961 \\
1962\end{array}$ & $\begin{array}{l}\text { Sept. 4, } 1961 \\
\text { Oct. 9, } 1961\end{array}$ & $\begin{array}{r}7.89 \\
10.00\end{array}$ & $\begin{array}{r}171 \\
1,480\end{array}$ & 1963 & $\begin{array}{ll}\text { May } 22,1963 \\
\text { Aug. } 17,1963 \\
\text { Sept.12, } 1963\end{array}$ & $\begin{array}{l}7.35 \\
8.21 \\
6.10\end{array}$ & $\begin{array}{l}186 \\
254 \\
114\end{array}$ \\
\hline
\end{tabular}

8-1370. Mukewater Creek subwatershed No. 9 near Trickham, Tex. (23)

Location.--Lat 31 $411^{\prime} 40^{\prime \prime}$, long $99^{\circ} 12^{\prime} 18^{\prime \prime}$, near center of dam on tributary to Sand Creek, 1.5 miles upstream from mouth, 4.5 miles southwest of Bangs, Brown County, and 7.1 miles north of Trickham, Coleman County.

Drainage area. $--4.02 \mathrm{sq} \mathrm{mi}$.

Gage.--Recording. Datum of gage is 1,500.01 ft above mean sea level, datum of 1929.

Remarks.--Peak discharge based on maximum inflow (average for 5 to 15-minute intervals), computed from outflow and change in reservoir contents, adjusted for rainfall on the reservoir surface during time of peak inflow. Only annual peaks are shown.

Peak stages and discharges

\begin{tabular}{|c|c|c|c|c|c|c|c|}
\hline $\begin{array}{r}\text { Water } \\
\text { year }\end{array}$ & Date & $\begin{array}{c}\text { Gage } \\
\text { height } \\
\text { (feet) }\end{array}$ & $\begin{array}{l}\text { Discharge } \\
\text { (cfs) }\end{array}$ & $\begin{array}{l}\text { water } \\
\text { year }\end{array}$ & Date & $\begin{array}{c}\text { Gage } \\
\text { height } \\
\text { (feet) }\end{array}$ & $\begin{array}{c}\text { Discharge } \\
\text { (cfs) }\end{array}$ \\
\hline $\begin{array}{r}a .1961 \\
1962\end{array}$ & $\begin{array}{lll}\text { June } & 5,1961 \\
\text { Oct. } & 9,1961\end{array}$ & - & $\begin{array}{r}1,440 \\
44 \\
\end{array}$ & 1963 & May 22,1963 & - & 186 \\
\hline
\end{tabular}

a Maximum for period January to September 1961 . 
8-1375. Mukewater Creek at Trickhan; Tex.(23)

Location. - Lat $31^{\circ} 36^{\prime}$, long $99^{\circ} 13^{\prime}$, on left bank at Trickham, Coleman County, $750 \mathrm{ft}$ upstream from bridge on State Farm Road 1176, 2.9 miles upstream from Hay Creek, and 6.9 miles upstream from mouth.

Drainage area. $--70.0 \mathrm{sq} \mathrm{ml}$.

Gage:--Recording. Datum of gage is 1,394.54 $\mathrm{ft}$ above mean sea level (State IIIighway Department bench mark).

Stage-discharge relation.--Defined by current-meter measurements below 6,000 cfs and by contracted-opening measurement at 15,000 cfs.

Bankfull stage. $--8 \mathrm{ft}$.

Historical data. - Maximum stage since at least 1919 occurred in 1927, from information by local resident.

Remarks.--Between 1961 and 1963, f1ve floodwater-retarding structures were bu11t in the basin upstream from this station. These structures have a total floodwaterretarding capacity of 2,960 acre-ft below the flood splliway crests, and partiy control the flow from 15.3 sq $\mathrm{m} 1$ above the station. Base for partial-duration series, $600 \mathrm{cfs}$.

Peak stages and discharges

\begin{tabular}{|c|c|c|c|c|c|c|c|}
\hline $\begin{array}{l}\text { Water } \\
\text { year }\end{array}$ & Date & $\begin{array}{c}\text { Gage } \\
\text { nelght } \\
\text { (feet) }\end{array}$ & $\begin{array}{c}\text { D1scharge } \\
(\text { cfs })\end{array}$ & $\begin{array}{l}\text { Water } \\
\text { year }\end{array}$ & Date & $\begin{array}{c}\text { Gage } \\
\text { nelght } \\
\text { (feet) }\end{array}$ & $\begin{array}{c}\text { D1scharge } \\
\text { (cfs) }\end{array}$ \\
\hline 1927 & - & 18 & - & 1957 & Apr. 19,19 & 5.06 & 855 \\
\hline 1951 & May 22,1951 & 11.40 & 4,900 & & $\begin{array}{l}\text { Apr. 23, } 1957 \\
\text { Apr. 26, } 1957\end{array}$ & $\begin{array}{r}5.21 \\
12.45\end{array}$ & $\begin{array}{r}915 \\
6,760\end{array}$ \\
\hline 1952 & $\begin{array}{ll}\text { Apr. } & 22,1952 \\
\text { May } 18, & 1952 \\
\text { May } 24, & 1952\end{array}$ & $\begin{array}{l}4.68 \\
4.69 \\
5.82\end{array}$ & $\begin{array}{r}708 \\
708 \\
1,140\end{array}$ & & $\begin{array}{lll}\text { May } & 11, & 1957 \\
\text { May } & 18,1957 \\
\text { May } & 24,1957\end{array}$ & $\begin{array}{l}8.17 \\
9.27 \\
6.25\end{array}$ & $\begin{array}{l}2,580 \\
3,430 \\
1,470\end{array}$ \\
\hline 1953 & Mar. 9, 1953 & 4.64 & 690 & 1958 & Aug. 24,1958 & 4.57 & 670 \\
\hline & May 12, 1953 & 5.25 & 920 & 1959 & $\begin{array}{l}\text { June } 2,1959 \\
\text { June } 4,1959\end{array}$ & $\begin{array}{r}5.10 \\
11.26\end{array}$ & $\begin{array}{r}947 \\
5.820\end{array}$ \\
\hline 1954 & $\begin{array}{l}\text { Oct. } 4,1953 \\
\text { Mar. 24, } 1954 \\
\text { May 11, } 1954\end{array}$ & $\begin{array}{l}6.79 \\
6.33 \\
4.68\end{array}$ & $\begin{array}{r}1,620 \\
1,360 \\
708\end{array}$ & & $\begin{array}{l}\text { June 26, } 1959 \\
\text { July 21, } 1959\end{array}$ & $\begin{array}{r}6.08 \\
11.90\end{array}$ & $\begin{array}{l}1,370 \\
6,620\end{array}$ \\
\hline 1955 & $\begin{array}{lrl}\text { May } & 10,1955 \\
\text { May } 18,1955 \\
\text { June } & 5,1955\end{array}$ & $\begin{array}{r}10.85 \\
8.52 \\
4.70\end{array}$ & $\begin{array}{r}4,320 \\
2,460 \\
727\end{array}$ & 1960 & $\begin{array}{l}\text { Oct. 4, } 1959 \\
\text { Jan. 5, } 1960 \\
\text { Jan. 14, } 1960\end{array}$ & $\begin{array}{l}5.57 \\
4.30 \\
4.27\end{array}$ & $\begin{array}{r}1,150 \\
620 \\
610\end{array}$ \\
\hline & $\begin{array}{l}\text { June } 7,1955 \\
\text { June } 9,1955 \\
\text { June } 15,1955\end{array}$ & $\begin{array}{l}5.62 \\
4.51 \\
7.54\end{array}$ & $\begin{array}{r}1,060 \\
671 \\
2,040\end{array}$ & 1961 & $\begin{array}{l}\text { June } 3,1961 \\
\text { June } 17,1961\end{array}$ & $\begin{array}{l}6.46 \\
5.87\end{array}$ & $\begin{array}{l}1,550 \\
1,280\end{array}$ \\
\hline & $\begin{array}{l}\text { July 18, } 1955 \\
\text { Sept.23, } 1955\end{array}$ & $\begin{array}{l}7.01 \\
6.71\end{array}$ & $\begin{array}{l}1,680 \\
1,560\end{array}$ & 1962 & Oct. 10,1961 & 2.94 & 172 \\
\hline 1956 & May 1,1956 & 15.83 & 15,000 & 1963 & $\begin{array}{lll}\text { May } & 20,1963 \\
\text { May } & 22,1963\end{array}$ & $\begin{array}{l}4.47 \\
5.98\end{array}$ & $\begin{array}{r}688 \\
1,340\end{array}$ \\
\hline 1957 & Mar. 20, 1957 & 4.44 & 628 & & $\begin{array}{l}\text { May } 30,1963 \\
\text { June } 16,1963 \\
\text { June } 17,1963 \\
\end{array}$ & $\begin{array}{l}4.37 \\
4.29 \\
4.46 \\
\end{array}$ & $\begin{array}{l}648 \\
616 \\
684 \\
\end{array}$ \\
\hline
\end{tabular}

Note:--Some peak discharges

8-1395. Deep Creek near Mercury, Tex.(23)

Location.- - Lat $31^{\circ} 24^{\prime} 10^{\prime \prime}$, long $99^{\circ} 071^{\prime} 15^{\prime \prime}$, near left bank on downstream side of bridge on Farm Road $502,1.5$ miles upstream from Dry Prong Deep Creek and 2.3 miles southeast of Mercury, McCulloch County.

Drainage area. $--43.9 \mathrm{sq} \mathrm{m} 1$.

Gage.--Nonrecording oct. I to Nov. 25, 1953; recording thereafter. Datum of gage is 1,325.64 $\mathrm{ft}$ above mean sea level, datum of 1929 .

Stage-discharge relation. - -Defined by current-meter measurements below 4,500 cfs and by slope-area measurements at 5,440 and $33,600 \mathrm{cfs}$.

Bankfull stage. $-14 \mathrm{ft}$.

Historlcal data.-Flood of July 23, 1938, was the highest since at least 1890, from information by local resident.

Remarks.--Between 1952 and 1953, five floodwater-retarding structures were built in the basin upstream from this station. These structures have total floodwater-retarding capacity of 5,730 acre-ft below the flood spillway crests, and partly control the flow f'rom 19.9 sq $\mathrm{ml}$ above the station.
Only annual peaks are shown.

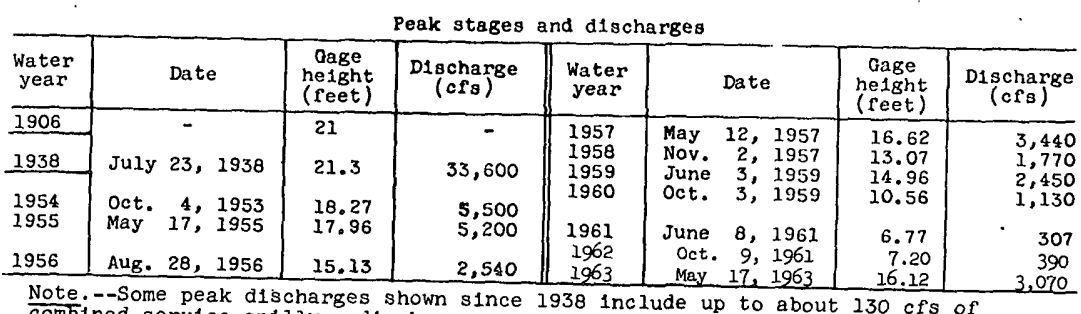

Note.--Some peak discharges shown since 1938 include up to about 130 cfs of 
8-1400. Deep Creek subwatershed No. 8 (Dry Prong Deep Creek) near Mercury, Tex. (23)

Location.--Lat $31^{\circ} 23^{\prime} 05^{\prime \prime}$, long $99^{\circ} 08^{\prime} 30^{\prime \prime}$, near center of dam on Dry Prong Deep Creek, 1.9 miles southeast of Mercury, McCulloch County, and 3.5 m1les upstream from mouth.

Dralnage area. $--4.32 \mathrm{sq} \mathrm{ml}$.

Gage.--Recording. Datum of gage is $1,377.13 \mathrm{ft}$ above mean sea level, datum of 1929 (levels by Soll Conservation Service).

Remarks.--Peak discharges based on maximum inflow (average for 15-minute interval) computed from outflow and change in reservolr contents, adjusted for rainfall on the reservolr surface during time of peak inflow. Base for partial-duration series, 100 cfs.

\begin{tabular}{|c|c|c|c|c|c|c|c|}
\hline $\begin{array}{l}\text { Water } \\
\text { year }\end{array}$ & Date & $\begin{array}{c}\text { aage } \\
\text { he1ght } \\
\text { (feet) }\end{array}$ & 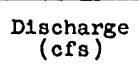 & $\begin{array}{l}\text { Water } \\
\text { year }\end{array}$ & Date & $\begin{array}{c}\text { Gage } \\
\text { he1ght } \\
\text { (feet) }\end{array}$ & $\begin{array}{c}\text { D1scharge } \\
(\mathrm{cfs})\end{array}$ \\
\hline 1952 & $\begin{array}{l}\text { Apr. } 18,1952 \\
\text { Apr. } 22,1952 \\
\text { May } 18,1952 \\
\text { May } 24,1952 \\
\text { Sept.11, } 1952\end{array}$ & $\begin{array}{l}\bar{z} \\
\bar{z}\end{array}$ & $\begin{array}{r}\text { ab500 } \\
\text { a120 } \\
\text { a114 } \\
\text { a146 } \\
\text { a252 }\end{array}$ & \multirow[t]{2}{*}{1957} & $\begin{array}{l}\text { Mar. } 20,1957 \\
\text { Apr. } 23,1957 \\
\text { Apr. } 26,1957 \\
\text { May } 3,1957 \\
\text { May } 11,1957\end{array}$ & $\begin{array}{l}\bar{z} \\
\bar{z} \\
=\end{array}$ & $\begin{array}{l}\mathrm{c} 258 \\
221 \\
\mathrm{c} 156 \\
107 \\
\mathrm{c} 130\end{array}$ \\
\hline 1953 & $\begin{array}{l}\text { Nov. } 9,1952 \\
\text { Nov. } 25,1952 \\
\text { Mar. }\end{array}$ & $\overline{-}$ & $\begin{array}{l}\text { al22 } \\
\text { a157 } \\
\text { a107 }\end{array}$ & & $\begin{array}{l}\text { May } 12,1957 \\
\text { May } 13,1957 \\
\text { May } 18,1957\end{array}$ & $\bar{z}$ & $\begin{array}{r}c 894 \\
\text { d260 } \\
654\end{array}$ \\
\hline & May 12,1953 & - & ab900 & \multirow[t]{2}{*}{1958} & $\begin{array}{l}\text { Nov. } 2,1957 \\
\text { Feb, } 22,1958\end{array}$ & - & $\begin{array}{l}521 \\
190\end{array}$ \\
\hline 1954 & 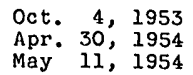 & $\overline{-}$ & $\begin{array}{r}1,570 \\
114 \\
277\end{array}$ & & $\begin{array}{l}\text { Mar. } 7,1958 \\
\text { Aug. } 23,1958\end{array}$ & - & $\begin{array}{l}157 \\
104\end{array}$ \\
\hline 1955 & $\begin{array}{l}\text { Oct. } 27,1954 \\
\text { Feb. } 4,1955 \\
\text { May } 17,1955 \\
\text { May } 18,1955\end{array}$ & $\begin{array}{l}\bar{z} \\
\overline{-}\end{array}$ & $\begin{array}{r}c 201 \\
c 126 \\
c 2,550 \\
c 1,270\end{array}$ & 1959 & $\begin{array}{r}\text { June } \\
\text { June } \\
\text { June } 1959 \\
\text { June } 26,1959 \\
\text { July } 21,1959 \\
\end{array}$ & $\begin{array}{l}- \\
\overline{-}\end{array}$ & $\begin{array}{l}332 \\
266 \\
221 \\
185\end{array}$ \\
\hline & $\begin{array}{l}\text { June } 5,1955 \\
\text { July 18, } 1955 \\
\text { Sept. } 23,1955\end{array}$ & $\overline{-}$ & $\begin{array}{r}c 294 \\
c 151 \\
1,440\end{array}$ & 1960 & $\begin{array}{lr}\text { Oct. } & 3,1959 \\
\text { Oct. } 13,1959 \\
\text { Jan. } & 5,1960\end{array}$ & - & $\begin{array}{r}\text { a } 323 \\
128 \\
143\end{array}$ \\
\hline 1956 & $\begin{array}{lrl}\text { May } & 1, & 1956 \\
\text { May } & 24, & 1956 \\
\text { Aug. } & 28,1956\end{array}$ & - & $\begin{array}{r}\text { c141 } \\
\text { c150 } \\
557\end{array}$ & $\begin{array}{l}1961 \\
1962 \\
1963\end{array}$ & $\begin{array}{l}\text { Sept.23, } 1960 \\
\text { Dec. } 7,1960 \\
\text { Nov. 2, } 1961 \\
\text { May } 5,1963\end{array}$ & $\overline{-}$ & $\begin{array}{r}274 \\
\text { e217 } \\
\text { b1.00 } \\
\text { e } 408 \\
\end{array}$ \\
\hline
\end{tabular}
Note.--Maximum discharge past structure during per1od, 56 cfs May 19, 1955.

8-1405. Dry Prong Deep Creek near Mercury, Tex. (23)

Location.--Lat $31^{\circ} 24^{\prime} 10^{\prime \prime}$, long $99^{\circ} 08^{\prime} 10^{\prime \prime}$, near center of span on downstream side of bridge on Farm Road 502, 1.3 miles southeast of Mercury, McCulloch County, 1.7 miles downstream from floodwater-retarding structure, and

1.8 miles upstream from mouth.

Drainage area. $--8.31 \mathrm{sq} \mathrm{m}$.

Gage.--Recording. Datum of gage 1s 1,339.02 ft above mean sea level, datum of 1929 .

Stage-discharge relation.--Defined by current-meter measurements.

Historlcal data.--Flood of May 17, 1955, 1s the highest since at least 1924 , from information by local resident.

Remarks.--In December 1951, one floodwater-retarding structure was built on the creek at a site 1.7 miles upstream from this station. This utructure has a total floodwater-retarding capacity of 1,410 acre-ft below flood splllway crest, and partiy controls the flow from $4.32 \mathrm{sq} \mathrm{ml}$ above this station. Only annual peaks are shown.

Peak stages and discharges

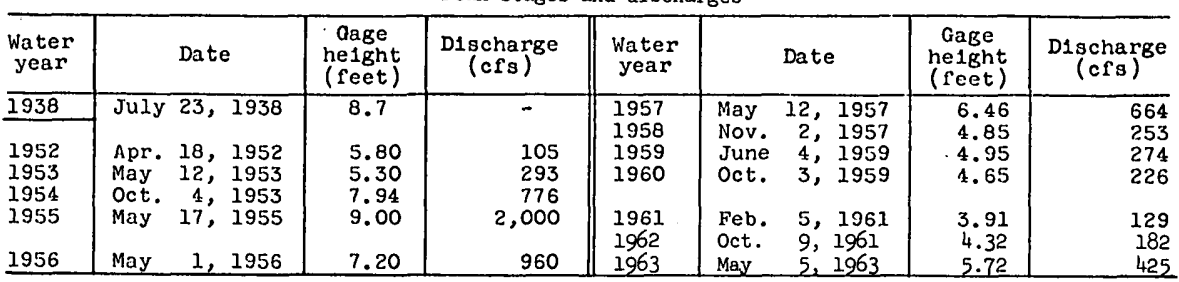


Location. - - Lat $30^{\circ} 17^{\prime} 49^{\prime \prime}$, long $97^{\circ} 43^{\prime} 36^{\prime \prime}$, on rlght bank 200 ft upstream from bridge at East 38 th Street at Austin, Travis County, 1.1 miles upstream from West Branch of Waller Creek, and 3.3 miles upstream from Colorado River.

Drainage area. $--2.31 \mathrm{sq} \mathrm{ml}$.

Gage.--Recording. Datum of gage is $555.44 \mathrm{ft}$ above mean sea level, datum of 1929 , Fort Worth supplementary adjustment of 1942.

Stage-discharge relation.--Defined by current-meter measurements.

Remarks. - Th1s station operated as research project for runoff from urban areas. Base for partial-duration series, 200 cfs.

\begin{tabular}{|c|c|c|c|c|c|c|c|}
\hline $\begin{array}{l}\text { Water } \\
\text { year }\end{array}$ & Date & $\begin{array}{c}\text { Cage } \\
\text { helght } \\
\text { (feet) }\end{array}$ & $\begin{array}{c}\text { Discharge } \\
(\mathrm{cfs})\end{array}$ & $\begin{array}{l}\text { Water } \\
\text { year }\end{array}$ & Date & $\begin{array}{c}\text { Gage } \\
\text { ne1ght } \\
\text { (feet) }\end{array}$ & $\begin{array}{c}\text { Discharge } \\
\text { (cfs) }\end{array}$ \\
\hline 1956 & May 1,1956 & 3.94 & a.108 & 1959 & $\begin{array}{ll}\text { July } 21, & 1959 \\
\text { Sept.23, } 1959\end{array}$ & $\begin{array}{l}4.81 \\
5.41\end{array}$ & $\begin{array}{l}287 \\
\text {. } \quad 468\end{array}$ \\
\hline \multirow[t]{6}{*}{1957} & Mar. 20, 1957 & 4.51 & 214 & & & & \\
\hline & $\begin{array}{l}\text { Apr. } 24,1957 \\
\text { Apr. } 26,1957\end{array}$ & $\begin{array}{l}4.56 \\
4.95\end{array}$ & $\begin{array}{l}225 \\
326\end{array}$ & 1960 & Oct. 4, 1959 & 4.67 & 251 \\
\hline & Apr. 28,1957 & 4.68 & 253 & 1961 & Oct. 16,1960 & 4.58 & 230 \\
\hline & May 26,1957 & 5.75 & 596 & & Oct. 18,1960 & 4.45 & 201 \\
\hline & June 3,1957 & 4.85 & 298 & & Oct. 29,1960 & 7.77 & 1,970 \\
\hline & June 12, 1957 & 5.50 & 500 & & $\begin{array}{l}\text { Feb. } 16,1961 \\
\text { June } 17,1961\end{array}$ & $\begin{array}{l}5.05 \\
5.88\end{array}$ & $\begin{array}{l}355 \\
676\end{array}$ \\
\hline \multirow[t]{6}{*}{1958} & Oct. 14, 1957 & 5.54 & 518 & & July 9,1961 & 5.68 & 690 \\
\hline & Feb. 22,1958 & 4.87 & 304 & & July 13, 1961 & 4.94 & 323 \\
\hline & $\begin{array}{l}\text { Apr. } 13,1958 \\
\text { Apr. 26, } 1958\end{array}$ & $\begin{array}{l}4.92 \\
5.39\end{array}$ & $\begin{array}{l}318 \\
464\end{array}$ & & July 17, 1961 & 404 & 323 \\
\hline & May 2, 1958 & 4.63 & 241 & 1962 & June 3,1962 & 6.00 & 805 \\
\hline & June 17,1958 & 4.91 & 315 & & June 10,1962 & 7.11 & 1,420 \\
\hline & July 6,1958 & 5.51 & 500 & & Aug. 25,1962 & 5.56 & 622 \\
\hline \multirow[t]{2}{*}{1959} & Apr. 8,1959 & 4.57 & 227 & & & & \\
\hline & July 20, 1959 & 4.57 & 227 & 1963 & $\begin{array}{l}\text { Oct. } 9,1962 \\
\text { June } 18,1963\end{array}$ & $\begin{array}{l}4.51 \\
4.72 \\
\end{array}$ & $\begin{array}{l}214 \\
263\end{array}$ \\
\hline
\end{tabular}

a Maximum for period Apr. 1 to Sept. 30, 1956.

8-1575. Waller Creek at 23d Street, Austin, Tex. (14)

Location.--Lat $30^{\circ} 17^{\prime} 08^{\prime \prime}$, long $97^{\circ} 44^{\prime} 01^{\prime \prime}$, on San Jacinto Boulevard, $50 \mathrm{ft}$ upstream from bridge on East $23 d$ Street at Austin, Travis County, and 2.1 miles upstream from Colorado RIver.

Drainage area. $--4.13 \mathrm{sq} \mathrm{ml}$.

Gage.--Recording. Datum of gage is $509.95 \mathrm{ft}$ above mean sea level, datum of 1929, Fort worth supplementary adjustment of 1942 .

Stage-discharge relation.--Defined by current-meter measurements .

Bankfull stage. $-12 \mathrm{ft}$.

Remarks.--Base for partial-duration series, 600 cfs.

Peak stages and discharges

\begin{tabular}{|c|c|c|c|c|c|c|c|c|}
\hline $\begin{array}{l}\text { Water } \\
\text { year }\end{array}$ & \multicolumn{2}{|r|}{ Date } & $\begin{array}{c}\text { Gage } \\
\text { he1ght } \\
\text { (feet) }\end{array}$ & $\begin{array}{c}\text { Discharge } \\
\text { (cfs) }\end{array}$ & $\begin{array}{l}\text { Water } \\
\text { year }\end{array}$ & Date & $\begin{array}{c}\text { Gage } \\
\text { helght } \\
\text { (feet) }\end{array}$ & $\begin{array}{c}\text { D1scharge } \\
(\mathrm{cfs})\end{array}$ \\
\hline 1951 & \multirow{2}{*}{\multicolumn{2}{|c|}{$\begin{array}{l}\text { June } 12,1951 \\
\text { Oct. } 23,1953\end{array}$}} & \multirow{2}{*}{ b8.0 } & 22,010 & \multirow{2}{*}{$\begin{array}{l}1957 \\
1958\end{array}$} & \multirow{4}{*}{$\begin{array}{ll}\text { June } 12, & 1957 \\
& \\
\text { Oct. } 14,1957 \\
\text { Feb. } 22 ; 1958 \\
\text { Apr. } 26,1958 \\
\text { May 2, } 1958 \\
\text { June } 17,1958 \\
\text { July } 6, & 1958\end{array}$} & \multirow{4}{*}{$\begin{array}{l}5.85 \\
4.75 \\
3.98 \\
5.47 \\
4.58 \\
4.24 \\
5.02\end{array}$} & \multirow{4}{*}{$\begin{array}{r}2,050 \\
1,120 \\
655 \\
1,700 \\
1,010 \\
804 \\
1,340\end{array}$} \\
\hline 1954 & & & & - & & & & \\
\hline $1955 c /$ & $\begin{array}{l}\text { May } \\
\text { May }\end{array}$ & $\begin{array}{ll}18, & 1955 \\
19, & 1955\end{array}$ & $\begin{array}{l}5.40 \\
4.17\end{array}$ & $\begin{array}{r}1,640 \\
762\end{array}$ & & & & \\
\hline 1956 & May & 1,1956 & 3.90 & 615 & & & & \\
\hline 1957 & $\begin{array}{l}\text { Mar. } \\
\text { Mar. } \\
\text { Apr. } \\
\text { Apr. } \\
\text { Apr. }\end{array}$ & $\begin{array}{l}11,1957 \\
20,1957 \\
22,1957 \\
24,1957 \\
26,1957 \\
28,1957\end{array}$ & $\begin{array}{l}3.93 \\
4.85 \\
3.92 \\
4.15 \\
4.80 \\
4.25\end{array}$ & $\begin{array}{r}630 \\
1,200 \\
625 \\
750 \\
1,160 \\
810\end{array}$ & 1959 & $\begin{array}{lr}\text { Oct. } 22, & 1958 \\
\text { Apr. } 8, & 1959 \\
\text { July 20, } & 1959 \\
\text { July 21, } & 1959 \\
\text { Sept.23, } & 1959\end{array}$ & $\begin{array}{l}4.29 \\
4.68 \\
4.60 \\
4.62 \\
5.71\end{array}$ & $\begin{array}{r}834 \\
1,080 \\
1,020 \\
1,030 \\
1,910\end{array}$ \\
\hline & $\begin{array}{l}\text { May } \\
\text { June }\end{array}$ & $\begin{array}{r}26,1957 \\
3,1957\end{array}$ & $\begin{array}{l}5.32 \\
3.94\end{array}$ & $\begin{array}{r}1,580 \\
635\end{array}$ & 1960 & Oct. 4,1959 & 4.11 & 726 \\
\hline
\end{tabular}

a Peak discharge determined by slope-area measurement half a mile downstream from gage.

b Annual peak only.

c January to September. 
COLORADO RIVER BASIN

Peak stages and discharges of Waller Creek at 23d Street; Aust1n, Tex.--Cont1nued

\begin{tabular}{c|c|r|r||r|r|r|r}
\hline $\begin{array}{c}\text { Water } \\
\text { year }\end{array}$ & Date & $\begin{array}{c}\text { Gage } \\
\text { he1ght } \\
\text { (feet) }\end{array}$ & $\begin{array}{c}\text { D1scharge } \\
\text { (cfs) }\end{array}$ & $\begin{array}{l}\text { Water } \\
\text { year }\end{array}$ & Date & $\begin{array}{c}\text { Gage } \\
\text { he1ght } \\
\text { (feet) }\end{array}$ & $\begin{array}{c}\text { D1scharge } \\
\text { (cf3) }\end{array}$ \\
\hline 1961 & Oct. 16, 1960 & 4.62 & 1,020 & 1962 & June 3, 1962 & 6.40 & 2,270 \\
& Oct. 29, 1960 & 7.96 & 3,710 & & June 10, 1962 & 5.70 & 1,700 \\
& Feb. 16, 1961 & 4.37 & 872 & & Aug. 25, 1962 & 6.04 & 1,980 \\
& June 12, 1961 & 4.05 & 692 & & Sept. 8, 1962 & 4.59 & 802 \\
& June 17, 1961 & 5.55 & 1,620 & & & & \\
& July 9, 1961 & 6.28 & 2,170 & 1963 & Oct. 1, 1962 & 4.47 & 932 \\
& July 12, 1961 & 5.70 & 1,730 & & June 18, 1963 & 4.70 & 1,070 \\
\hline
\end{tabular}

8-1600. Dry Creek at Buescher Lake, near Smithville, Tex.(14)

Location.--Lat $30^{\circ} 02^{\prime} 35^{\prime \prime}$, long $97^{\circ} 09^{\prime} 20^{\prime \prime}$, on left bank, 225 ft upstream from dam in Bastrop-Buescher State Park, 1.9 miles upstream from mouth, and 2.2 miles north of Smithville, Bastrop County.

Drainage area. $--1.48 \mathrm{sq} \mathrm{ml}$.

Gage.--Recording. Datum of gage is $327.86 \mathrm{ft}$ above mean sea level, datum of 1929 .

Stage-discharge relation.--Inflow into lake only, and is computed on basis of change in reservoir contents plus flow over splllway. Generally, peak Inflow is computed as the average inflow for a period of less than 30 minutes, unadjusted for rainfall on reservoir surface during time of peak inflow.

Remarks.--Only annual peaks are shown.

Peak stages and discharges

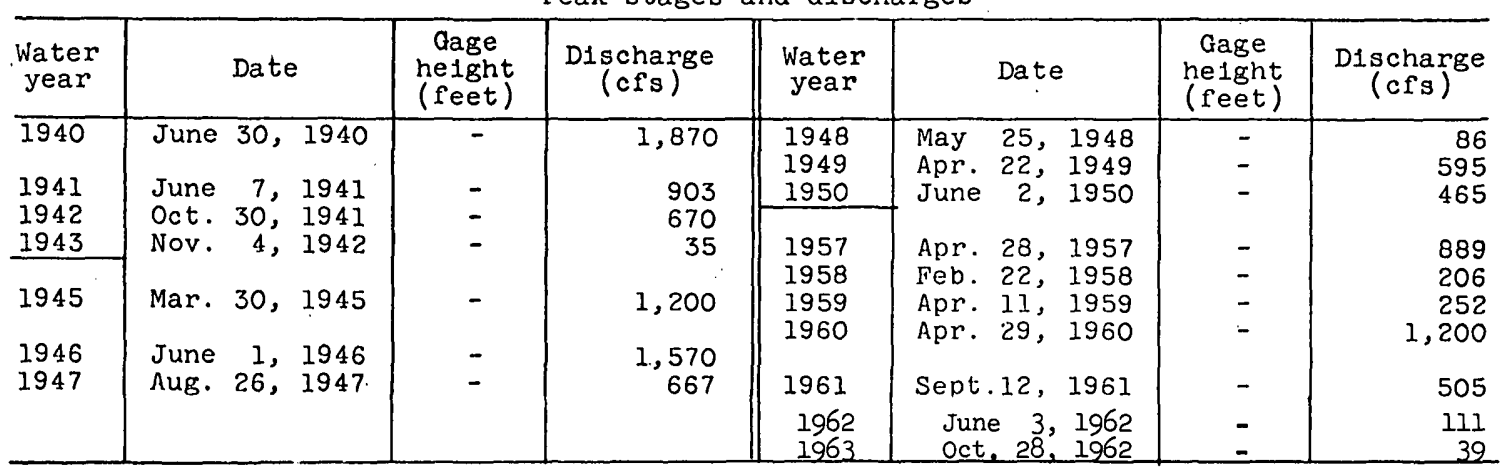


Location.--Lat $29^{\circ} 26^{\prime}$, long $96^{\circ} 57^{\prime}$, at downstream side of bridge on U. S. Highway 77 in Hallettsville, Lavaca County, and 0.4 mile upstream from Texas and New Orleans Railroad Co. bridge.

Drainage area.--101 sq $\mathrm{mi}$.

Gage.--Recording. Datum of gage is $186.72 \mathrm{ft}$ above mean sea level, datum of 1929.

Stage-discharge relation.--Defined by current-meter measurements below 23,000 cfs and extended on basis of slope-area measurement at 93,100 cfs.

Bankfull stage.--29 ft.

Historical data.-Flood of June 30, 1940, reached highest stage since at least 1840 , and the flood of July 16, 1936, was second highest in the period beginning about 1870, from information by local resident.

Remarks.--Extensive channel improvements were made in 1959. Base for partial-duration series, 2,300 cfs.

\begin{tabular}{|c|c|c|c|c|c|c|c|}
\hline $\begin{array}{l}\text { Water } \\
\text { year }\end{array}$ & Date & $\begin{array}{c}\text { Gage } \\
\text { height } \\
\text { (feet) }\end{array}$ & $\begin{array}{l}\text { Discharge } \\
\text { (cfs) }\end{array}$ & $\begin{array}{l}\text { Water } \\
\text { year }\end{array}$ & Date & $\begin{array}{l}\text { Gage } \\
\text { height } \\
\text { (feet) }\end{array}$ & $\begin{array}{l}\text { Discharge } \\
\text { (cfs) }\end{array}$ \\
\hline 1936 & July 16, 1936 & 32.8 & 28,300 & 1949 & Apr. 20, 1949 & 16.90 & 2,750 \\
\hline 1940 & $\begin{array}{l}\text { June } 30,1940 \\
\text { July } 3,1940\end{array}$ & $\begin{array}{l}40.60 \\
19.00\end{array}$ & $\begin{array}{r}93,100 \\
2,320\end{array}$ & & $\begin{array}{l}\text { Apr. 22, } 1949 \\
\text { Apr. 25, } 1949\end{array}$ & $\begin{array}{l}20.28 \\
21.54\end{array}$ & $\begin{array}{l}4,920 \\
5,860\end{array}$ \\
\hline \multirow[t]{11}{*}{194.1} & $\begin{array}{l}\text { Oct. } 29,1940 \\
\text { Nov. } 5,1940 \\
\text { Nov. } 24,1940\end{array}$ & $\begin{array}{l}20.76 \\
21.72 \\
29.36\end{array}$ & $\begin{array}{r}3,220 \\
3,980 \\
17,000\end{array}$ & 1950 & $\begin{array}{ll}\text { Oct. 22, } 1949 \\
\text { May 27, } 1950 \\
\text { June 2, } 1950\end{array}$ & $\begin{array}{l}16.68 \\
19.17 \\
18.80\end{array}$ & $\begin{array}{l}2,640 \\
4,130 \\
3,900\end{array}$ \\
\hline & $\begin{array}{l}\text { Dec. } 11,1940 \\
\text { Dec. } 15,1940\end{array}$ & $\begin{array}{l}23.70 \\
21.94\end{array}$ & $\begin{array}{l}6,160 \\
4,180\end{array}$ & 1951 & June 12, 1951 & 19.85 & 4,600 \\
\hline & $\begin{array}{l}\text { Dec. } 20,1940 \\
\text { Jan. } 14,1941 \\
\text { Mar. } 6,1941\end{array}$ & $\begin{array}{l}19.75 \\
20.60 \\
21.75\end{array}$ & $\begin{array}{l}2,650 \\
3,080 \\
4,080\end{array}$ & 1952 & $\begin{array}{ll}\text { Apr. } 12, & 1952 \\
\text { May } 27, & 1952\end{array}$ & $\begin{array}{l}19.30 \\
26.94\end{array}$ & $\begin{array}{r}4,220 \\
11,400\end{array}$ \\
\hline & $\begin{array}{l}\text { Mar. } 18,1941 \\
\text { Apr. } 7,1941\end{array}$ & $\begin{array}{l}19.22 \\
19.40\end{array}$ & $\begin{array}{l}2,400 \\
2,480\end{array}$ & 1953 & May 14, 1953 & 15.15 & 1,480 \\
\hline & Apr. 22, 1941 & 22.20 & 4,480 & 1954 & Apr. 8, 1954 & 19.10 & 3,260 \\
\hline & Apr. 26, 1941 & 19.73 & 2,600 & & & & \\
\hline & May $3,194.2$ & 23.33 & 5,680 & 1955 & Feb. 4, 1955 & 26.84 & 10,200 \\
\hline & May 5,1941 & 21.35 & 3,680 & & May 18,1955 & 27.18 & 10,800 \\
\hline & May 11,1941 & 22.65 & 4,880 & & Aug. 20, 1955 & 22.23 & 5,150 \\
\hline & May 22, 1941 & 24.55 & 7,400 & & & & \\
\hline & June $11,194.1$ & 19.75 & 2,650 & 1956 & Feb. 8, 1956 & 14.02 & 1,310 \\
\hline \multirow[t]{4}{*}{1942} & Oct. 31, 1941 & 19.60 & $.4,420$ & 1957 & Mar. 31, 1957 & 22.62 & 4,880 \\
\hline & Apr. 8, 1942 & 28.43 & 14,500 & & Apr. 21, 1957 & 25.58 & 8,500 \\
\hline & Apr. 24, 1942 & 19.90 & 4,630 & . & Apr. 27, 1957 & 25.20 & 7,900 \\
\hline & July 6, 1942 & 24.70 & 8,860 & & $\begin{array}{l}\text { Apr. 29, } 1957 \\
\text { May 27, } 1957\end{array}$ & $\begin{array}{l}26.15 \\
21.00\end{array}$ & $\begin{array}{l}9,460 \\
3,720\end{array}$ \\
\hline 1943 & May 25,1943 & 16.22 & 2,500 & & Sept.25, 1957 & 22.82 & 5,060 \\
\hline \multirow[t]{4}{*}{1944} & Jan. 29, 1944 & 16.24 & 2,500 & 1958 & Oct. 15, 1957 & 30.55 & 20,600 \\
\hline & Mar. 15,1944 & 26.61 & 11,000 & & Oct. 22, 1957 & 19.10 & 3,440 \\
\hline & May 28,1944 & 17.82 & 3,330 & & Nov. 22, 1957 & 20.17 & 4,140 \\
\hline & Aug. 30,1944 & 21.50 & 5,860 & & Feb. 22, 1958 & 22.86 & 6,210 \\
\hline \multirow[t]{3}{*}{194.5} & Jan. 18, 1945 & 22.36 & 6,590 & 1959 & Apr. 10, 1959 & 23.40 & 6,660 \\
\hline & $\begin{array}{l}\text { Mar. } 30,1945 \\
\text { Apr. } 1,194.5\end{array}$ & $\begin{array}{l}21.45 \\
16.85\end{array}$ & $\begin{array}{l}5,780 \\
2,800\end{array}$ & & June 4, 1959 & 18.37 & 4,120 \\
\hline & & & & 1960 & Oct. 31, 1959 & 18.88 & 4,370 \\
\hline \multirow[t]{3}{*}{1946} & $\begin{array}{l}\text { Feb. } 18,1946 \\
\text { June } 1,1946\end{array}$ & $\begin{array}{l}20.95 \\
16.90\end{array}$ & $\begin{array}{l}5,700 \\
2,800\end{array}$ & & June 25, 1960 & 15.49 & 3,760 \\
\hline & June $\overrightarrow{8}, 1946$ & 20.60 & 5,080 & 1961 & Oct. 16,1960 & 24.2 & 13,300 \\
\hline & Aug. 29, 1946 & 19.90 & 4,630 & & Oct. 18,1960 & 28.4 & 29,500 \\
\hline \multirow[t]{4}{*}{1947} & Nov. 4,1946 & 20.20 & 4,850 & & Nov. 22, 1960 & 15.0 & 2,310 \\
\hline & Mar. 18,1947 & 21.00 & 5,460 & & Feb. 16,1961 & 12.78 &, 390 \\
\hline & Apr. 13,1947 & 18.78 & 3,900 & & June 18,1961 & 27.6 & 25,500 \\
\hline & May 24,1947 & 18.90 & 3,960 & & Sept.12, 1961 & 27.94 & 27,000 \\
\hline \multirow[t]{3}{*}{1948} & May 27,1948 & 18.45 & 3,650 & 1962 & Apr. 27, 1962 & 16.83 & 4,000 \\
\hline & & & & & June 3,1962 & 15.92 & 3,370 \\
\hline & & & & 1963 & Feb. 18,1963 & 14.21 & 2,340 \\
\hline
\end{tabular}


Location.--Lat $30^{\circ} 06^{\prime} 00^{\prime \prime}$, long $99^{\circ} 17^{\prime} 00^{\prime \prime}$, on right bank, 1.3 miles upstream from Henderson Branch, $3.4 \mathrm{~m}$ liles northwest of Ingram, Kerr County, 3.8 miles upstream from mouth, and $9.2 \mathrm{miles}$ northwest of Kerrville.

Drainage area. - - $1.15 \mathrm{sq} \mathrm{mi}$.

Gage.--Recording. Datum of gage is 1,721.30 ft above mean sea level, datum of 1929.

Stage-discharge relation.--Defined by current-meter measurements below 1,800 cfs. Oct. 10, 1953, to 0ct. 3, 1959, affected by dam below gage and extended above 1,800 cfs to 4,800 cfs by weir

formula study. For other periods, extended above $1,800 \mathrm{cfs}$ on basis of slope-area measurements a.t 9,100 and 16,000 cfs and conveyance study to $95,900 \mathrm{cfs}$.

Bankfull stage.--25 ft.

Historical data.--Maximum stage since at least 1852 occurred July 2, 1932, from information by local residents. Flood of June 14, 1935, reached a stage of about 31 or $32 \mathrm{ft}$, from information by local residents.

Remarks.--Base for partial-duration series, 50 cfs.

Peak stages and discharges

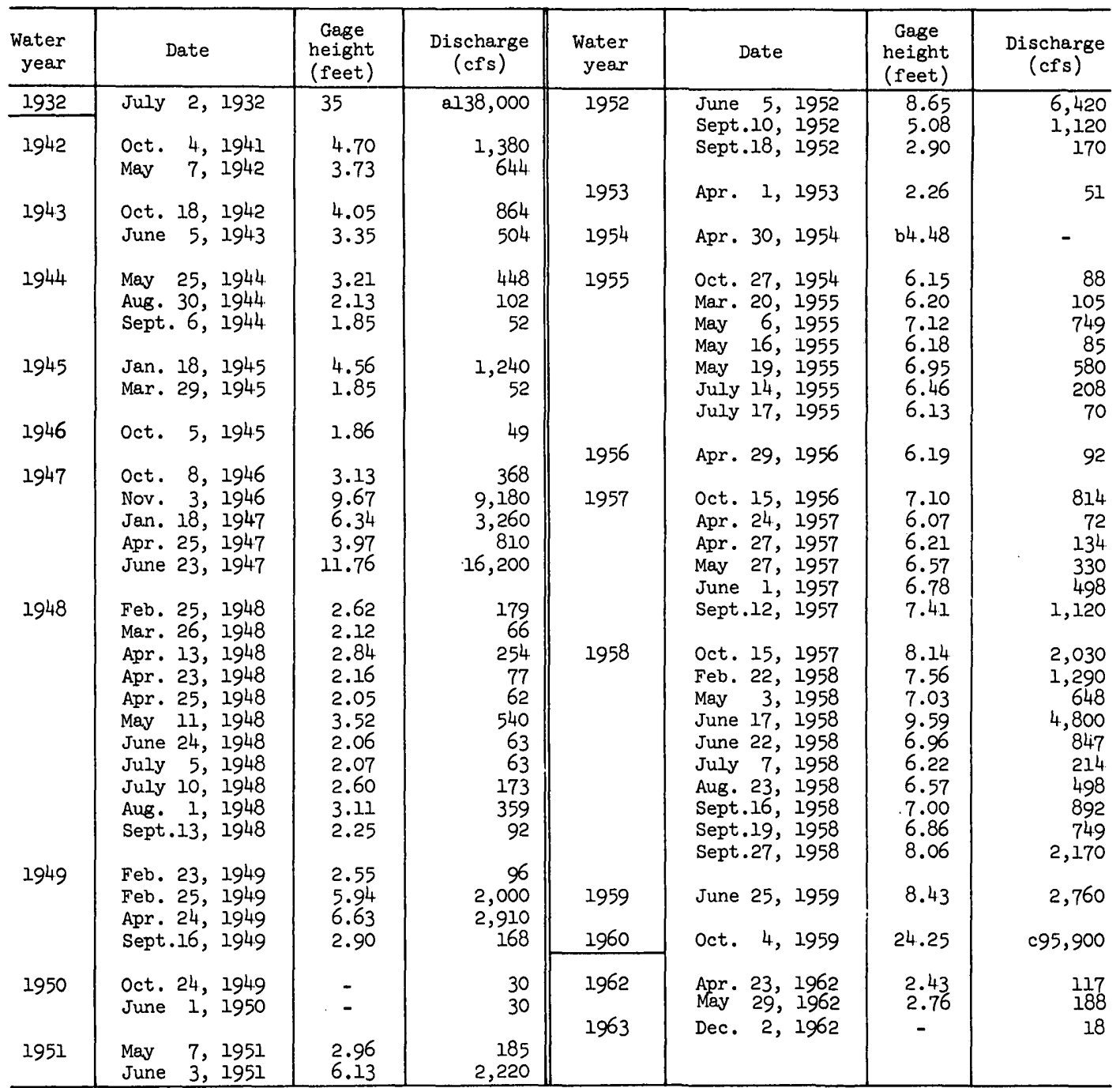

a Result of slope-area measurement 6 of 7 miles upstream from gage.

b Backwater from dam.

c Annual peak only. 
8-1676. Rebecca Creek near Spring Branch, Tex. (15)

Location.--Lat $29^{\circ} 55^{\prime} 08^{\prime \prime}$, long $98^{\circ} 22^{\prime} 09^{\prime \prime}$, on right bank $72 \mathrm{ft}$ upstream from private road crossing, $2.8 \mathrm{miles}$ upstream from mouth, and 4.0 miles northeast of Spring Branch, Comal County.

Drainage area.--11.0 sq $\mathrm{mi}$.

Gage.--Recording. Datum of gage is $985.55 \mathrm{ft}$ above mean sea level, datum of 1929.

Stage-discharge relation.--Defined by current-meter measurements below 20 cfs and by critical-depth measurement of peak flow of $4,340 \mathrm{cfs}$.

Bankfull stage. --52 ft.

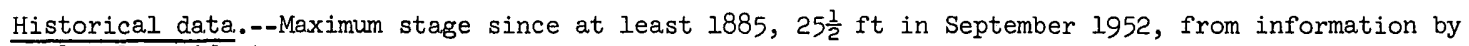
local residents.

Remarks.--Ba.se for partial-duration series, $50 \mathrm{cfs}$. Rain gage at site.

Peak stages and discharges

\begin{tabular}{|c|c|c|c|c|c|c|c|}
\hline $\begin{array}{r}\text { Water } \\
\text { year }\end{array}$ & Date & $\begin{array}{c}\text { Gage } \\
\text { height } \\
\text { (feet) }\end{array}$ & $\begin{array}{c}\text { Discharge } \\
(\mathrm{cfs})\end{array}$ & $\begin{array}{r}\text { Water } \\
\text { year }\end{array}$ & Date & $\begin{array}{c}\text { Gage } \\
\text { height } \\
\text { (feet) }\end{array}$ & $\begin{array}{c}\text { Discharge } \\
\text { (cfs) }\end{array}$ \\
\hline $\begin{array}{l}1961 \\
1962\end{array}$ & $\begin{array}{l}\text { Oct. } 18,1960 \\
\text { Oct. } 29,1960 \\
\text { Apr. } 27,1962\end{array}$ & $\begin{array}{l}4.54 \\
6.18 \\
2.12\end{array}$ & $\begin{array}{r}1,490 \\
4,340 \\
3.8\end{array}$ & 1963 & $\begin{array}{l}\text { Apr. } 5,1963 \\
\text { May } 27,1963 \\
\text { Aug. } 15,1963\end{array}$ & $\begin{array}{l}6.20 \\
2.96 \\
2.57\end{array}$ & $\begin{array}{r}4,340 \\
179 \\
57\end{array}$ \\
\hline
\end{tabular}




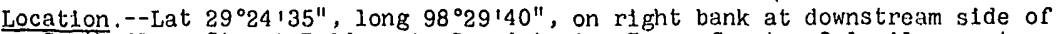
South Alamo Street Bridge, in San Antonio, Bexar County, 2.1 miles upstream from San Pedro Creek.

Drainage area $--42 \mathrm{sq} \mathrm{ml}$, approximately.

Gage.--Nonrecording at site 1.9 miles upstream Feb. 28, 1916, to Apr. 7, 1920; recording thereafter. Datum of gage is $612.26 \mathrm{ft}$ above mean sea level, datum of 1929. Datum of gages prior to Apr. 8, 1920, unknown.

Stage-discharge relation.--Defined by current-meter measurements below 2,200 $\mathrm{cf}$ 's and by slope-area measurement at 15,300 cfs.

H1storical data.--Maximum stage since 1819, that of Sept. 10, 1921; flood of July 5, 1819, equaled or exceeded that of Sept. 10, 1921. Highest recorded stage prior to 1921 occurred Oct. 23,1914 , at Commerce Street Bridge, relation to present gage not known.

Remarks.--Only annual peaks are shown. Since 1926, floodflow regulated by 01mos flood-control reservolr (capac1ty, 15,500 acre-ft), $8 \frac{1}{2}$ m1les upstream.

\begin{tabular}{|c|c|c|c|c|c|c|c|}
\hline $\begin{array}{l}\text { Water } \\
\text { year }\end{array}$ & Date & $\begin{array}{c}\text { Gage } \\
\text { he1ght } \\
\text { (feet) }\end{array}$ & $\begin{array}{c}\text { D1scharge } \\
\text { (cfs) }\end{array}$ & $\begin{array}{l}\text { Water } \\
\text { year }\end{array}$ & Date & $\begin{array}{l}\text { Gage } \\
\text { nelght } \\
\text { (feet) }\end{array}$ & $\begin{array}{c}\text { Discharge } \\
\text { (cfs) }\end{array}$ \\
\hline 1915 & oct. 23,1914 & 14.0 & 4,600 & 1943 & Oct. 4,1942 & 8.16 & 2,350 \\
\hline $\begin{array}{l}1916 \\
1917\end{array}$ & $\begin{array}{l}\text { Sept.25, } 1914 \\
\text { Mar.24, } 25, \text { May } 20\end{array}$ & $\begin{array}{l}9.4 \\
1.8\end{array}$ & $\begin{array}{r}2,650 \\
147\end{array}$ & 19 & Dec. 4,1944 & 7.21 & 1,820 \\
\hline $\begin{array}{l}1918 \\
1919 \\
1920\end{array}$ & $\begin{array}{l}1917 \\
\text { May } 5,1918 \\
\text { Sept.15, } 1919 \\
\text { Oct. 16, } 1919 \\
\end{array}$ & $\begin{array}{r}-.7 \\
7.7 \\
7.8 \\
\end{array}$ & $\begin{array}{r}- \\
2,380 \\
2,430 \\
\end{array}$ & $\begin{array}{l}1946 \\
1947 \\
1948 \\
1949\end{array}$ & $\begin{array}{l}\text { Sept.27, } 1946 \\
\text { Nov. } 10,1946 \\
\text { Aug. } 26,1948 \\
\text { June } 25,1949\end{array}$ & $\begin{array}{r}15.32 \\
5.85 \\
10.60 \\
7.85\end{array}$ & $\begin{array}{r}5,740 \\
984 \\
2,840 \\
1,640\end{array}$ \\
\hline 1921 & Sept.10, 1921 & 20.14 & 15,300 & & & & \\
\hline $\begin{array}{l}1923 \\
1924 \\
1925\end{array}$ & $\begin{array}{lll}\text { July } & 21,1923 \\
\text { May } 26, & 1924 \\
\text { May } & 10,1925\end{array}$ & $\begin{array}{l}3.5 \\
4.5 \\
4.5\end{array}$ & $\begin{array}{l}390 \\
648 \\
641\end{array}$ & $\begin{array}{l}1951 \\
1952 \\
1953 \\
1954 \\
1955\end{array}$ & $\begin{array}{l}\text { June } 3,1951 \\
\text { Sept.18, } 1952 \\
\text { Sept. 4, } 1953 \\
\text { June 26, } 1954 \\
\text { Feb. 4, } 1955\end{array}$ & $\begin{array}{l}8.2 .2 \\
3.47 \\
7.55 \\
5.05 \\
5.23\end{array}$ & $\begin{array}{r}1,800 \\
363 \\
1,610 \\
758 \\
810\end{array}$ \\
\hline $\begin{array}{l}1926 \\
1927 \\
1928 \\
1929 \\
\end{array}$ & $\begin{array}{l}\text { Apr. } 20,1926 \\
\text { June } 14,1927 \\
\text { June 2, } 1928 \\
\text { May 24, } 1929\end{array}$ & $\begin{array}{l}8.3 \\
5.18 \\
4.93 \\
4.32\end{array}$ & $\begin{array}{r}1,940 \\
845 \\
755 \\
588\end{array}$ & $\begin{array}{l}1956 \\
1957 \\
1958\end{array}$ & $\begin{array}{lll}\text { May } & 15, & 1956 \\
\text { May } & 27, & 1957 \\
\text { June } & 22, & 1958\end{array}$ & $\begin{array}{l}6.63 \\
9.23 \\
7.34\end{array}$ & $\begin{array}{l}1,230 \\
1,850 \\
1,210\end{array}$ \\
\hline 1940 & June 29,1940 & 4.45 & 1,040 & 1960 & Aug. 15, 1960 & 10.12 & 2,360 \\
\hline $\begin{array}{l}1941 \\
1942\end{array}$ & $\begin{array}{l}\text { Apr. } 28,1941 \\
\text { Sept. } 4,1942\end{array}$ & $\begin{array}{l}5.76 \\
5.90\end{array}$ & $\begin{array}{l}1,470 \\
1.680\end{array}$ & $\begin{array}{l}1961 \\
1962 \\
1963\end{array}$ & $\begin{array}{l}\text { July } 22,1961 \\
\text { Sept. 6, } 1962 \\
\text { Sept.12, } 1963\end{array}$ & $\begin{array}{r}12.20 \\
5.60 \\
6.50\end{array}$ & $\begin{array}{l}3,410 \\
1,140 \\
1,500\end{array}$ \\
\hline
\end{tabular}

8-1785. San Pedro Creek at San Antonio, Tex.(15)

Location.--Lat $29^{\circ} 25^{\prime}$, long $98^{\circ} 30^{\prime}$, at M1ssouri, Kansas and Texas Ra1lway culvert, $200 \mathrm{ft}$ below Arsenal Street in San Antonio, Bexar County, threequarters of a mile upstream from Apache and Alazan Creck, ond $2 \frac{1}{2}$ miles upstream from San Antonio River.

Dratnage area. $--2.64 \mathrm{sq} \cdot \mathrm{m} 1$.

Gage.--Nonrecording prior to Mar. 14, 1921; recording thereafter. Datum of gage unknown. At Commerce Strect Bridge, half a m1lc upstream at different datum July 19, 1916, to Mar. 13, 1921.

Stage-discharge relation.--Defined by current-meter measurements below $200 \mathrm{cfs}$ and extended above on basis of Kutler's formula.

Bankfull stage. $-7 \mathrm{ft}$.

IIIstorical data. - Flood of Sept. 9, 1921, at San Antonio greatly exceeded all floods of which there is any information except the flood of July 5,1819 which probably equaled if not exceeded it.

Remarks.--only annual peaks are shown.

Peak stages and discharges

\begin{tabular}{|c|c|c|c|c|c|c|c|c|}
\hline $\begin{array}{l}\text { Water } \\
\text { year }\end{array}$ & Date & $\begin{array}{l}\text { Cage } \\
\text { nelght } \\
\text { (reet) }\end{array}$ & $\begin{array}{c}\text { Discharge } \\
(\mathrm{cfs})\end{array}$ & $\begin{array}{l}\text { Water } \\
\text { year }\end{array}$ & & Date & $\begin{array}{l}\text { Gage } \\
\text { he1ght } \\
\text { (feet) }\end{array}$ & $\begin{array}{c}\text { Discharge } \\
(\mathrm{cfs})\end{array}$ \\
\hline 2914 & October 1913 & 10.2 & - & \multirow{4}{*}{$\begin{array}{l}1922 \\
1923 \\
1924 \\
1925 \\
1926 \\
1927 \\
1928 \\
1929 \\
\end{array}$} & \multirow{4}{*}{\multicolumn{2}{|c|}{$\begin{array}{lr}\text { May } & 2,1922 \\
\text { Aug. } 28, & 1923 \\
\text { Apr. } 25, & 1924 \\
\text { May } 10, & 1925 \\
& \\
\text { Apr. } 20, & 1926 \\
\text { June } 15, & 1927 \\
\text { Mar. } 9, & 1928 \\
\text { May } 23, & 1929 \\
\end{array}$}} & \multirow{4}{*}{$\begin{array}{l}1.25 \\
3.55 \\
6.38 \\
4.8 \\
6.40 \\
5.00 \\
6.79 \\
5.00 \\
\end{array}$} & \multirow{4}{*}{$\begin{array}{r}788 \\
404 \\
1,070 \\
680 \\
\\
1,070 \\
728 \\
1,170 \\
728 \\
\end{array}$} \\
\hline $\begin{array}{l}1916 \\
1917 \\
1918\end{array}$ & $\begin{array}{l}\text { Sept.25, } 1916 \\
\text { Oct.16, } 1916 \\
\text { Apr. 5, } 1918\end{array}$ & $\begin{array}{l}6.25 \\
3.5 \\
4.80\end{array}$ & $\begin{array}{r}2700 \\
145 \\
380\end{array}$ & & & & & \\
\hline $\begin{array}{l}1919 \\
1920\end{array}$ & $\begin{array}{l}\text { Sept.15, } 1919 \\
\text { Oct. 16, } 1919\end{array}$ & $\begin{array}{l}4.45 \\
3.40 \\
\end{array}$ & $\begin{array}{l}335 \\
170 \\
\end{array}$ & & & & & \\
\hline 1921 & Scpt. 9,1921 & 8.60 & b2, 020 & & & & & \\
\hline
\end{tabular}

a Max1mum July 20 to Sept. 30, 1916; probably maximum for year.

c. K. McDonald of offlce of Englneers, elghth corps area. 
8-1791. Red Bluff Creek near Pipe Creek, Tex. (15)

Location.--Lat $29^{\circ} 40^{\prime} 48^{\prime \prime}$, long $98^{\circ} 57^{\prime} 20^{\prime \prime}$, on left bank 0.8 mile upstream from bridge on county road, 1.7 miles downstream from Pipe Creek, 1.9 miles upstream from mouth, and 3.2 miles south of town of Pipe Creek, Bandera County.

Drainage area. $--56.3 \mathrm{sq} \mathrm{mi}$.

Gage.--Recording, Datum of gage is 1,107.2 ft above mean sea level, unadjusted.

Stage-discharge relation.--Defined by current-meter measurements below 2,000 cPs and above by a slope-area measurement of 46,900 cfs.

Bankfull stage. $--23 \mathrm{ft}$.

Historical data.--Maximum stage known since at least 1905, about $17 \mathrm{ft}$ in July 1937.

Remarks.--Base for partial-duration series, 1,000 cfs.

Peak stages and discharges

\begin{tabular}{|c|c|c|c|c|c|c|c|}
\hline $\begin{array}{r}\text { Water } \\
\text { year }\end{array}$ & Date & $\begin{array}{c}\text { Gage } \\
\text { height } \\
\text { (feet) }\end{array}$ & $\begin{array}{c}\text { Discharge } \\
\text { (cfs) }\end{array}$ & $\begin{array}{r}\text { Water } \\
\text { year }\end{array}$ & Date & $\begin{array}{c}\text { Gage } \\
\text { height } \\
\text { (feet) }\end{array}$ & $\begin{array}{c}\text { Discharge } \\
\text { (cfs) }\end{array}$ \\
\hline 1957 & $\begin{array}{lll}\text { Mar. } 20,1957 \\
\text { Apr. 22, } 1957 . \\
\text { Apr. 28, } 1957 \\
\text { May 14, } 1957 \\
\text { May 27, } 1957 \\
\text { June 1, } 1957\end{array}$ & $\begin{array}{l}6.76 \\
6.50 \\
7.64 \\
8.59 \\
8.08 \\
8.44\end{array}$ & $\begin{array}{l}1,270 \\
1,120 \\
1,870 \\
2,700 \\
2,240 \\
2,510\end{array}$ & $\begin{array}{l}1959 \\
1960\end{array}$ & $\begin{array}{l}\text { June 26, } 1959 \\
\text { Aug. 15, } 1960 \\
\text { Oct. 29, } 1960 \\
\text { Anr. 23, }\end{array}$ & $\begin{array}{r}11.00 \\
10.70 \\
4.16 \\
3.10\end{array}$ & $\begin{array}{r}5,500 \\
5,110 \\
162\end{array}$ \\
\hline 1958 & $\begin{array}{ll}\text { May } 2, & 1958 \\
\text { June 22, } 1958 \\
\text { Sept. 7, } 1958\end{array}$ & $\begin{array}{r}11.08 \\
6.40 \\
7.60\end{array}$ & $\begin{array}{l}5,630 \\
1,040 \\
1,830\end{array}$ & 1963 & $\begin{array}{l}\text { Oct. 28, } 1962 \\
\text { Apr. 28, } 1963\end{array}$ & $\begin{array}{l}6.46 \\
6.87\end{array}$ & $\begin{array}{l}1,050 \\
1,300\end{array}$ \\
\hline
\end{tabular}

\section{8-1824. Calaveras Creek subwatershed No. 6 near Elmendorf, Tex. (15)}

Location.--Lat $29^{\circ} 22^{\prime} 53^{\prime \prime}$, long $98^{\circ} 17^{\prime} 34^{\prime \prime}$, near center of dam on Chupaderas Creek, tributary to Calaveras Creek, 0.4 mile north of Sayer, 9.1 miles north of Elmendorf, Bexar County, and 9.2 miles upstream from mouth.

Drainage area. --7.01 sq $\mathrm{mi}$.

Gage---Recording. Datum of gage is $516.06 \mathrm{ft}$ above mean sea level, datum of 1929 (levels by U. S. Soil Conservation Service).

Remarks.--Peak discharge ba.sed on maximum inflow (average for 15-minute interval), computed from outflow and change in reservoir contents, adjusted for rainfall on the reservoir surface during time of peak inflow. Only annual peaks are shown.

\begin{tabular}{|c|c|c|c|c|c|c|c|}
\hline \multicolumn{8}{|c|}{ Peak stages and discharges } \\
\hline $\begin{array}{r}\text { Water } \\
\text { year }\end{array}$ & Date & $\begin{array}{c}\text { Gage } \\
\text { height } \\
\text { (feet) }\end{array}$ & $\begin{array}{l}\text { Discharge } \\
\text { (cfs) }\end{array}$ & $\begin{array}{l}\text { Water } \\
\text { year }\end{array}$ & Date & $\begin{array}{c}\text { Gage } \\
\text { height } \\
\text { (feet) }\end{array}$ & $\begin{array}{c}\text { Discharge } \\
(\mathrm{cfs})\end{array}$ \\
\hline $\begin{array}{l}1957 \\
1958 \\
1959 \\
1960\end{array}$ & $\begin{array}{l}\text { Sept.25, } 1957 \\
\text { Ma.y 3, } 1958 \\
\text { Apr. 11, } 1959 \\
\text { Oct. 4, } 1959\end{array}$ & $\begin{array}{l}- \\
- \\
-\end{array}$ & $\begin{array}{r}3,750 \\
1,880 \\
252 \\
419\end{array}$ & $\begin{array}{l}1961 \\
1962 \\
1963\end{array}$ & $\begin{array}{c}\text { June 18, } 1961 \\
\text { Nov. 13, } 1961 \\
-\end{array}$ & $\begin{array}{l}- \\
-\end{array}$ & $\begin{array}{l}800 \\
385 \\
(a)\end{array}$ \\
\hline
\end{tabular}

a Unknown. 
Location. - - Lat $29^{\circ} 15^{\prime} 30^{\prime \prime}$, long $98^{\circ} 17^{\prime} 30^{\prime \prime}$, near center of span on downstream side of bridge on U.S. Highway $181,2.5$ miles east of Elmendorf, Bexar County, 5 miles upstream from mouth, and 10 miles southeast from city $11 \mathrm{mits}$ of San Antonio.

Drainage area. $--77.2 \mathrm{sq}, \mathrm{mi}$.

Gage.--Recording. Datum of gage is $406.45 \mathrm{ft}$ above mean sea level, datum of 1929, supplementary adjustment of 1943 .

Stage-discharge relation.--Defined by current-meter measurements. Subject to seasonal shif's due to heavy vegetal growth.

Historical data.--Flood of Sept. 29, 1946, was the highest since at least 1860.

Remarks.--During the period 1954-58, nine floodwater-retarding structures were built in the basin above this station. These structures have a total floodwater-retarding capacity of 13,250 acre-ft below the flood splilway crests, and partly control the flow from $37.1 \mathrm{sq} \mathrm{ml}$ above the station. Only annual peaks are shown.

\begin{tabular}{|c|c|c|c|c|c|c|c|}
\hline $\begin{array}{l}\text { Water } \\
\text { year }\end{array}$ & Date & $\begin{array}{c}\text { Gage } \\
\text { helght } \\
\text { (feet) }\end{array}$ & $\begin{array}{c}\text { D1scharge } \\
(\text { cfs })\end{array}$ & $\begin{array}{l}\text { Water } \\
\text { year }\end{array}$ & Date & $\begin{array}{c}\text { Gage } \\
\text { he1ght } \\
\text { (feet) }\end{array}$ & $\begin{array}{c}\text { Discharge } \\
\text { (cfs) }\end{array}$ \\
\hline 1946 & Sept.29, 1946 & 35 & - & $\begin{array}{l}1958 \\
1959\end{array}$ & $\begin{array}{l}\text { Feb. } 21,1958 \\
\text { May } 24,1959\end{array}$ & $\begin{array}{l}14.74 \\
14.98\end{array}$ & $\begin{array}{l}2,010 \\
1,400\end{array}$ \\
\hline 1955 & May 16,1955 & 11.73 & 568 & 1960 & Oct. 4, 1959 & 14.22 & 1,370 \\
\hline $\begin{array}{l}1956 \\
1957\end{array}$ & $\begin{array}{l}\text { Oct. } 11,1955 \\
\text { Sept.25, } 1957\end{array}$ & $\begin{array}{l}12.07 \\
21.83\end{array}$ & $\begin{array}{r}607 \\
5,310\end{array}$ & $\begin{array}{l}1961 \\
1962 \\
1963\end{array}$ & $\begin{array}{ll}\text { Oct. } 29,1960 \\
\text { June 2, } 1962 \\
\text { Nov. 27, } 1962\end{array}$ & $\begin{array}{c}13.69 \\
- \\
11.34\end{array}$ & $\begin{array}{r}1,140 \\
1,030 \\
733 \\
\end{array}$ \\
\hline
\end{tabular}

Note.--Peak discharges shown since 1957 include up to about 200 cfs combined flow from floodwaterretarding structures.

8-1870. Escondido Creek subwatershed No. 1 near Kenedy, Tex. (16)

Location. - - Lat $28^{\circ} 47^{\prime}$, long $97^{\circ} 54^{\prime}$, near center of dam on unnamed fork of Panther Creek, $500 \mathrm{ft}$ upstream from State H1ghway 72 and 3 miles southwest of Kenedy, Karnes County.

Drainage area. $--3.29 \mathrm{sq} \mathrm{ml}$.

Gage.--Recording. Datum of gage is $350.00 \mathrm{ft}$ above mean sea level, datum of 1929 (levels by Soll Conservation Service).

Remarks.--Peaks are based on maximum inflow (average for 15-minute interval), computed from outflow and change in reservolr contents, adjusted for rainfall on the reservoir surface during time of peak inflow. No adjustment made for reservolr losses. Base for partial-duration sertes, $100 \mathrm{cfs}$.

Peak stages and discharges

\begin{tabular}{|c|c|c|c|c|c|c|c|}
\hline $\begin{array}{l}\text { Water } \\
\text { year }\end{array}$ & Date & $\begin{array}{c}\text { Qage } \\
\text { nelght } \\
\text { (feet) }\end{array}$ & $\begin{array}{c}\text { D1scharge } \\
(\text { cfs })\end{array}$ & $\begin{array}{l}\text { Water } \\
\text { year }\end{array}$ & Date & $\begin{array}{c}\text { Gage } \\
\text { he1ght } \\
\text { (feet) }\end{array}$ & $\begin{array}{c}\text { Discharge } \\
(\text { cfs })\end{array}$ \\
\hline$\overline{1955}$ & $\begin{array}{lll}\text { July } 12, & 1955 \\
\text { Aug. } 11, & 1955 \\
\text { Aug. } 14, & 1955\end{array}$ & $\begin{array}{l}- \\
-\end{array}$ & $\begin{array}{r}986 \\
2,100 \\
105\end{array}$ & 1958 & $\begin{array}{lrr}\text { Nov. } & 22,1957 \\
\text { Jan. } & 12,1958 \\
\text { Feb. } 22, & 1958 \\
\text { May } & 3, & 1958\end{array}$ & $\begin{array}{l}- \\
- \\
-\end{array}$ & $\begin{array}{r}139 \\
348 \\
288 \\
1,700\end{array}$ \\
\hline 1956 & June 19,1956 & - & 486 & 1959 & Sept.29, 1959 & - & 181 \\
\hline 1957 & $\begin{array}{ll}\text { Mar. } 11, & 1957 \\
\text { Mar. } 31,1957 \\
\text { Apr. 14, } 1957 \\
\text { Apr. 20, } 1957 \\
\text { Apr. 27, } 1957 \\
\text { May 13, } 1957 \\
\text { May 27, } 1957 \\
\text { June 1, } 1957 \\
\text { Sept.22, } 1957 \\
\text { Sept.23, } 1957\end{array}$ & $\begin{array}{l}\overline{-} \\
\overline{-} \\
\overline{-} \\
\overline{-} \\
\overline{-} \\
-\end{array}$ & $\begin{array}{r}146 \\
205 \\
250 \\
1,300 \\
404 \\
2560 \\
a 1,810 \\
418 \\
359 \\
268\end{array}$ & $\begin{array}{l}1961 \\
1962 \\
1963\end{array}$ & $\begin{array}{l}\text { Oct. } 4,1959 \\
\text { Jan. 14, } 1960 \\
\text { July 17, } 1960 \\
\text { Aug. 28, } 1960 \\
\text { Aug. 29, } 1960 \\
\text { Aug. } 30,1960 \\
\begin{array}{l}\text { Oct. 25, } 1960 \\
\text { June 1, } 1962 \\
\text { Nov. 27, } 1962\end{array}\end{array}$ & $\begin{array}{l}- \\
\overline{-} \\
- \\
- \\
- \\
\overline{-}\end{array}$ & $\begin{array}{r}653 \\
166 \\
a 817 \\
121 \\
114 \\
139 \\
\mathrm{~b} 4,990 \\
\mathrm{~b} 745 \\
\mathrm{~b} 1,300\end{array}$ \\
\hline
\end{tabular}

a Not adjusted for rainfall on water surface.

b Annual peak only. 
Location.--Iat $28^{\circ} 49^{\prime}$, long $97^{\circ} 52^{\prime}$, near center of span on downstream side of bridge on U.S. Highway 181 in northwest edge of Kenedy, Karnes County, $3 \frac{1}{2}$ miles upstream from Dry Escondido Creek, and $8 \frac{1}{2}$ miles upstream from mouth.

Drainage area. $--82.2 \mathrm{sq} \mathrm{ml}$, of which $36.5 \mathrm{sq} \mathrm{mi}$ is above flood-detention structures.

Gage.--Recording. Datum of gage is $246.40 \mathrm{ft}$ above mean sea level, datum of 1929 .

Stage-discharge relation.--Defined by current-meter measurements below 4,400 cf's and extended above.

Historical data.--Maximum stage since at least 1887 , that of Aug. 29, 1946 , from information by local residents.

Remarks.--Flow from $36.5 \mathrm{sq} \mathrm{ml}$ above station is partly controlled by 10 floodwater-detention reservolrs, completed between Sept. 21, 1954, and Feb. 17, 1957, with a total combined capacity of 13,300 acre-ft below flood splilway crests. Only annual peaks are shown.

\begin{tabular}{|c|c|c|c|c|c|c|c|}
\hline $\begin{array}{l}\text { Water } \\
\text { year }\end{array}$ & Date & $\begin{array}{c}\text { Qage } \\
\text { helght } \\
\text { (feet) }\end{array}$ & $\underset{(\mathrm{cfs})}{\text { Discharge }}$ & $\begin{array}{l}\text { Water } \\
\text { year }\end{array}$ & Date & $\begin{array}{c}\text { Gage } \\
\text { nelght } \\
\text { (feet) }\end{array}$ & $\begin{array}{l}\text { D1scharge } \\
\text { (cfs) }\end{array}$ \\
\hline 1946 & Aug. 29,1946 & 24.2 & 12,300 & 1958 & 3,1958 & 19.78 & 3,330 \\
\hline 1955 & Aug. 31, 1955 & 19.82 & 3,370 & 1960 & Oct. 4,1959 & 17.26 & 1,680 \\
\hline $\begin{array}{l}1956 \\
1957\end{array}$ & $\begin{array}{l}\text { Sept. 2, } 1956 \\
\text { Sent.23. } 1957\end{array}$ & $\begin{array}{r}16.77 \\
\cdot 18.45\end{array}$ & $\begin{array}{l}1,250 \\
2.160\end{array}$ & $\begin{array}{l}1961 \\
1962 \\
1963 \\
\end{array}$ & $\begin{array}{l}\text { Oct. } 25,1960 \\
\text { June } 2,1962 \\
\text { Dec. } 2,1962 \\
\end{array}$ & $\begin{array}{l}23.55 \\
14.35 \\
17.28 \\
\end{array}$ & $\begin{array}{r}10,700 \\
688 \\
1,380 \\
\end{array}$ \\
\hline
\end{tabular}

Note.--Peak discharge shown for oct. 25, 1960 includes undetermined amount of flow over emergency splilways of floodwater-retarding structures. Peak discharges shown since 1955 include up to about $350 \mathrm{cfs}$ of combined service splliway discharge.

\section{8-1880. Dry Escond1do Creek near Kenedy, Tex. (16)}

Location.--Lat $28^{\circ} 52^{\prime}$, long $97^{\circ} 50^{\prime}$, at bridge on State Farm Road $792,3.5$ miles north of Kenedy, Karnes County, 4.0 miles upstream from Escondido Creek, and 4.0 miles southeast of Karnes City.

Drainage area. $--9.43 \mathrm{sq} \mathrm{mi}$.

Gage.--Recording. Datum of gage is $276.55 \mathrm{ft}$ above mean sea level, datum of 1929.

Stage-discharge relation.--Defined by current-meter measurements.

Historical data.--Flood of May 18, 1953, was the highest since at least 1906 , from information by local resident.

Remarks.--Flow from $8.43 \mathrm{sq} \mathrm{ml}$ above station is partly controlled since Jan. 31 , 1958 , by one flood-detention structure. Only annual peaks are shown.

Peak stages and discharges

\begin{tabular}{|c|c|c|c|c|c|c|c|c|}
\hline $\begin{array}{l}\text { Water } \\
\text { year }\end{array}$ & & Date & $\begin{array}{c}\text { Gage } \\
\text { he1ght } \\
\text { (feet) }\end{array}$ & $\begin{array}{c}\text { Discharge } \\
(\text { cfs })\end{array}$ & $\begin{array}{l}\text { water } \\
\text { year }\end{array}$ & Date & $\begin{array}{c}\text { Gage } \\
\text { helght } \\
\text { (feet) }\end{array}$ & $\begin{array}{c}\text { Discharge } \\
(\mathrm{cfs})\end{array}$ \\
\hline 1953 & May & 18,1953 & 16 & $=$ & 1957 & Apr. 21,1957 & 10.53 & $\begin{array}{l}706 \\
155\end{array}$ \\
\hline 1955 & May & 12,1955 & 7.55 & 290 & 1959 & Oct. 30, 1958 & 5.49 & 13 \\
\hline 1956 & & 3, 1956 & 7.22 & 215 & & & & \\
\hline
\end{tabular}

Note.--Maximum outflow from station 8-1870 was 10 cfs during period of record. 
8-1960. Dry Frio River near. Reagan Wells, Tex. (22)

Location.--Lat $29^{\circ} 30^{\prime}$, long $99^{\circ} 47^{\prime}$, on right bank 1,000 ft upstream from Aldine School, 2 miles upstream from Rock Creek, and 4 miles southeast of Reagan Wells, Uvalde County.

Drainage area. - - $117 \mathrm{sq} \mathrm{mi}$.

Gage.--Recording. Datum of gage is $1,335.2 \mathrm{ft}$ above mean sea level, adjustment unknown (levels by Ground Water Branch).

Stage-discharge relation.--Defined by current-meter measurements below 720 cfs and by slope-area measurements at 11,400 and 64,700 cfs.

Historical data.--Floods of 1880 and June 14, 1935, are the highest since at least 1875, from information by local resident.

Remarks.--Only annual peaks are shown.

\begin{tabular}{|c|c|c|c|c|c|c|c|}
\hline $\begin{array}{r}\text { Water } \\
\text { year }\end{array}$ & Date & $\begin{array}{c}\text { Gage } \\
\text { height } \\
\text { (feet) }\end{array}$ & $\begin{array}{l}\text { Discharge } \\
\quad(\mathrm{cfs})\end{array}$ & $\begin{array}{r}\text { Water } \\
\text { year }\end{array}$ & Date & $\begin{array}{c}\text { Gage } \\
\text { height } \\
\text { (feet) }\end{array}$ & $\begin{array}{l}\text { Discharge } \\
\quad(\mathrm{cfs})\end{array}$ \\
\hline 1880 & - & 33 & - & 1956 & Oct. 2, 1955 & 2.67 & $\begin{array}{r}34 \\
8,000\end{array}$ \\
\hline 1935 & June 14, 1935 & 26 & 264,700 & $\begin{array}{l}1951 \\
1958 \\
1959\end{array}$ & $\begin{array}{l}\text { Sept.19, } 1958 \\
\text { Sept.23, } 1959\end{array}$ & $\begin{array}{r}12.0 \\
13.72 \\
6.20\end{array}$ & $\begin{array}{r}10,700 \\
2,020\end{array}$ \\
\hline $\begin{array}{l}1953 \\
1954\end{array}$ & $\begin{array}{l}\text { Sept. 1, } 1953 \\
\text { May 25, } 1954\end{array}$ & $\begin{array}{r}2.81 \\
14.12\end{array}$ & $\begin{array}{r}211 \\
11,400\end{array}$ & 1960 & Oct. 4, 1959 & 13.3 & 10,000 \\
\hline 1955 & Sept.24, 1955 & 18.68 & 23,200 & $\begin{array}{l}1961 \\
1962 \\
1963\end{array}$ & $\begin{array}{l}\text { June } 18,1961 \\
\text { Oct. } 27,1961 \\
\text { May } 6,1963\end{array}$ & $\begin{array}{l}15.0 \\
2.74 \\
2.98\end{array}$ & $\begin{array}{r}13,200 \\
55 \\
118 \\
\end{array}$ \\
\hline
\end{tabular}

a From slope-area measurement at site 2.6 miles upstream. 
Location.--Lat $29^{\circ} 34^{\prime}$, long $99^{\circ} 15^{\prime}$, on left bank $460 \mathrm{ft}$ downstream from Ranch Road 462 low-water crossing, 6.2 mlles southeast of Tarpley, Bandera County, and 16.7 miles northwest of Hondo.

Drainage area. $--86.2 \mathrm{sq} \mathrm{ml}$.

Gage.--Recording. Datum of gage is 1,169.1 ft above mean sea level, adjustment unknown (Magnolia 011 Co. bench mark).

Stage-discharge relation.--Defined by current-meter measurements below $360 \mathrm{cfs}$ and by slope-area measurements at $3,340,18,600$, and $69,800 \mathrm{cfs}$.

Historical data.--Maximum stage since at least 1907, that of June 17, 1958; second highest, that in July 1932 .

Remarks.--Only annual peaks are shown.

\begin{tabular}{c|c|c|c||c|c|c|c}
\hline $\begin{array}{c}\text { Water } \\
\text { year }\end{array}$ & Date & $\begin{array}{c}\text { Gage } \\
\text { helght } \\
\text { (feet) }\end{array}$ & $\begin{array}{c}\text { Discharge } \\
\text { (cfs) }\end{array}$ & $\begin{array}{c}\text { Water } \\
\text { year }\end{array}$ & Date & $\begin{array}{c}\text { Gage } \\
\text { he1ght } \\
\text { (feet) }\end{array}$ & $\begin{array}{c}\text { D1scharge } \\
\text { (cfs) }\end{array}$ \\
\hline 1932 & July 1932 & 26.0 & 58,500 & 1958 & June 17, 1958 & 28.2 & 69,800 \\
\hline 1953 & Sept. 4, 1953 & 7.77 & 3,340 & 1959 & Apr. 7, 1959 & 9.20 & 5,200 \\
1954 & May 24, 1954 & 15.46 & 18,600 & 1960 & Oct. 4, 1959 & 10.10 & 6,640 \\
1955 & Mar. 20, 1955 & 6.02 & 1,570 & 1961 & June 18, 1961 & - & 6,500 \\
1956 & Sept. 6, 1956 & 4.20 & 1962 & June 1, 1962 & 4.18 & 550 \\
1957 & Sept.22, 1957 & 17.8 & 510 & 1963 & Apr. 28, 1963 & 3.08 & 196 \\
\hline
\end{tabular}

8-2015. Seco Creek at Miller Ranch, near Utopia, Tex. (22)

Location.--Lat $29^{\circ} 34^{\prime}$, long $99^{\circ} 24^{\prime}$, on right bank $200 \mathrm{ft}$ upstream from county road crossing, 4.2 miles downstream from Ca.scade Creek, and 8 miles southeast of Utopia, Uvalde County.

Drainage area. $--43.1 \mathrm{sq} \mathrm{mi}$.

Gage.--Recording. Datum of gage is $1,265.8 \mathrm{ft}$ above mean sea level, adjustment unknown (Magnolia Oil Co. bench mark).

Stage-discharge relation.--Defined by current-meter measurements below 160 cfs and above by a. slope-area measurement of $52,600 \mathrm{cfs}$.

Historical da.ta.--Maximum stage since at least 1901, $16.4 \mathrm{ft}$ June 17, 1958, from floodmarks (discharge, 52,600 cfs).

Remarks.--Base for partial-duration series, 1,000 cfs.

Peak stages and discharges

\begin{tabular}{|c|c|c|c|c|c|c|c|}
\hline $\begin{array}{l}\text { Water } \\
\text { year }\end{array}$ & Date & $\begin{array}{c}\text { Gage } \\
\text { height } \\
\text { (feet) }\end{array}$ & $\begin{array}{c}\text { Discharge } \\
\text { (cfs) }\end{array}$ & $\begin{array}{l}\text { Water } \\
\text { year }\end{array}$ & Da.te & $\begin{array}{c}\text { Gage } \\
\text { height } \\
\text { (feet) }\end{array}$ & $\begin{array}{c}\text { Discharge } \\
\text { (cfs) }\end{array}$ \\
\hline 1961 & June 18,1961 & 4.36 & 1,760 & $\begin{array}{l}1962 \\
1963\end{array}$ & $\begin{array}{lll}\text { June } & 1,1962 \\
\text { May } & 6, & 1963\end{array}$ & $\begin{array}{l}2.40 \\
2.88\end{array}$ & $\begin{array}{r}87 \\
279\end{array}$ \\
\hline
\end{tabular}




\section{8-2020. Seco Creek near Utopla, Tex.(22)}

Location.--Lat $29^{\circ} 33^{\prime}$, long $99^{\circ} 24^{\prime}$, on right bank half a mile downstream from county road crossing, 7.6 miles upstream from Bartz Spring Creek, and

9 miles southeast of Utopia, Uvalde County.

Drainage area. $--53.2 \mathrm{sq} \mathrm{ml}$.

Gage.--Recording. Datum of gage 1s 1,245.8 $\mathrm{ft}$ above mean sea level, adjustment unknown (Magnolia 011 Co. bench mark).

Stage-discharge relation.--Defined by current-meter measurements below $290 \mathrm{cfs}$ and by slope-area measurements at $1,910,9,040$, and $52,600 \mathrm{cfs}$.

Remarks.--Only annual peaks are shown.

Peak stages and discharges

\begin{tabular}{|c|c|c|c|c|c|c|c|}
\hline $\begin{array}{l}\text { Water } \\
\text { year }\end{array}$ & Date & $\begin{array}{l}\text { Gage } \\
\text { nelght } \\
\text { (feet) }\end{array}$ & $\begin{array}{c}\text { Discharge } \\
\text { (cfs) }\end{array}$ & $\begin{array}{l}\text { Water } \\
\text { year }\end{array}$ & Date & $\begin{array}{c}\text { Gage } \\
\text { helght } \\
\text { (feet) }\end{array}$ & $\begin{array}{c}\text { Discharge } \\
\text { (cfs) }\end{array}$ \\
\hline 1935 & May 1935 & & 40,000 & 1957 & Sept.22, 1957 & 11.63 & 12,100 \\
\hline $\begin{array}{l}1953 \\
1954 \\
1955\end{array}$ & $\begin{array}{ll}\text { Sept. } 2, & 1953 \\
\text { May } 24, & 1954 \\
\text { May } 19, & 1955\end{array}$ & $\begin{array}{c}5.88 \\
10.3 \\
4.23\end{array}$ & $\begin{array}{r}a 1,930 \\
b 9,040 \\
633\end{array}$ & $\begin{array}{l}1959 \\
1960\end{array}$ & $\begin{array}{l}\text { Apr. } 7,1959 \\
\text { Aug. } 15,1960\end{array}$ & $\begin{array}{l}4.27 \\
4.87\end{array}$ & $\begin{array}{r}657 \\
1,040\end{array}$ \\
\hline 1956 & July 3,1956 & 3.74 & 393 & 1961 & June 18, & 13 & U \\
\hline
\end{tabular}

a Result of slope-area measurement at gage.

$b$ Result of slope-area measurement three-quarters of a mile upstream.

\section{8:2025. Seco Creek near D'Hanis, Tex.(15)}

Location.--Lat $29^{\circ} 29^{\prime}$, long $99^{\circ} 23^{\prime}$, on right bank a quarter of a mile downstream from concrete dam and road crossing at Woodward Ranch headquarters, 2.8 miles upstream from Bartz Spring Creek, and 12.8 miles northwest of D'Hanis, Medina County.

Dralnage area. $--87.4 \mathrm{sq} \mathrm{m} 1$.

Gage.--Recording. Datum of gage is 1,142.8 ft above mean sea level (levels by Ground Water Branch).

Stage-discharge relation.--Defined by current-meter measurements below 250 cfs and by slope-area measurements at $2,090,8,110$, and 72,000 cfs (adjusted to present sitel.

Historical data.--Maximum stage since at least 1866, that in May 1935. Flood of Aug. 31, 1894, second highest prior to June 17, 1958, from information by local residents.

Remarks.--Only annual peaks are shown.

Peak stages and discharges

\begin{tabular}{|c|c|c|c|c|c|c|c|}
\hline $\begin{array}{l}\text { Water } \\
\text { year }\end{array}$ & Date & $\begin{array}{c}\text { Gage } \\
\text { helght } \\
\text { (feet) }\end{array}$ & $\begin{array}{c}\text { D1scharge } \\
(\text { cfs })\end{array}$ & $\begin{array}{l}\text { Water } \\
\text { year }\end{array}$ & Date & $\begin{array}{l}\text { Gage } \\
\text { helght } \\
\text { (feet) }\end{array}$ & $\begin{array}{c}\text { Discharge } \\
(\mathrm{cfs})\end{array}$ \\
\hline 1894 & Aug. 31, 1894 & 16 & 33,000 & 1956 & 3,1956 & 3.09 & 680 \\
\hline 1935 & May 1935 & 26.2 & - & 1958 & June 17,1958 & $\begin{array}{l}11.18 \\
20.8\end{array}$ & 72,000 \\
\hline $\begin{array}{l}1953 \\
1954 \\
1955 \\
\end{array}$ & $\begin{array}{lr}\text { Sept. } 2, & 1953 \\
\text { May 24, } 1954 \\
\text { Mar. 20, } 1955 \\
\end{array}$ & $\begin{array}{l}5.03 \\
9.30 \\
4.62 \\
\end{array}$ & $\begin{array}{l}2,090 \\
8,110 \\
1,730 \\
\end{array}$ & $\begin{array}{l}1959 \\
1960 \\
1961 \\
\end{array}$ & $\begin{array}{l}\text { Oct. } 22,1958 \\
\text { Oct. } 4,1959 \\
\text { June } 18,1961\end{array}$ & $\begin{array}{l}4.21 \\
4.27 \\
6.46 \\
\end{array}$ & $\begin{array}{r}918 \\
966 \\
3,370 \\
\end{array}$ \\
\hline
\end{tabular}


8-3656. McKelligon Canyon at El Paso, Tex. (24)

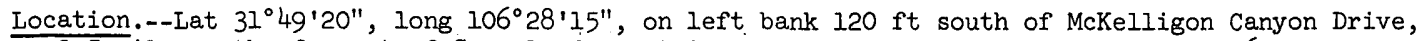
$0.5 \mathrm{mile}$ south of crest of Sugarloaf Mountain, 0.2 mile west of Albama Avenue, 1.6 miles west of U. S. Highway 54, and 4.5 miles north of El Paso post office.

Drainage area. $--2.3 \mathrm{sq} \mathrm{mi}$, approximately.

Gage.--Recording. Altitude of gage is 4,257.33 ft above mean sea level (levels by city of El Paso). Stage-discharge relation.--Based on culvert measurement of peak flow of $76 \mathrm{cfs}$.

Remarks.--No flow except Sept. 11, 12, 1958. Flood flow controlled by four small reservoirs upstream, with a total capacity of about 95 acre-feet.

Peak stages and discharges

\begin{tabular}{c|c|c|c||c|c|c|c}
\hline $\begin{array}{c}\text { Water } \\
\text { year }\end{array}$ & Da.te & $\begin{array}{c}\text { Gage } \\
\text { height } \\
\text { (feet) }\end{array}$ & $\begin{array}{c}\text { Discharge } \\
\text { (cfs) }\end{array}$ & $\begin{array}{c}\text { Water } \\
\text { year }\end{array}$ & Date & $\begin{array}{c}\text { Gage } \\
\text { height } \\
\text { (feet) }\end{array}$ & $\begin{array}{c}\text { Discharge } \\
\text { (cfs) }\end{array}$ \\
\hline al.958 & Sept.11, 1958 & - & 76 & & & & \\
\hline
\end{tabular}

a Period June to September 1958.

Note.--No flow to Sept. 30, 1963.

8-3658. Government ditch a.t El Paso, Tex. (24)

Location.--Lat $31^{\circ} 47^{\prime} 02^{\prime \prime}$, long 106 $26^{\circ} 04^{\prime \prime}$, at intersection of Montana and Houston Streets, 2 miles northeast of the business center of El Paso.

Drainage area.--6.4 sq mi, approximately.

Gage.--Recording. Altitude of gage is 3,740 ft (from topographic map).

Stage-discharge relation.--Defined by current-meter measurements below $148 \mathrm{cfs}$ and extended above on basis of slope-area measurement at $550 \mathrm{cfs}$.

Remarks.--Base for partial-duration series, $100 \mathrm{cfs}$.

Peak stages and discharges

\begin{tabular}{|c|c|c|c|c|c|c|c|}
\hline $\begin{array}{r}\text { Water } \\
\text { year } \\
\end{array}$ & Date & $\begin{array}{c}\text { Gage } \\
\text { height } \\
\text { (feet) }\end{array}$ & $\begin{array}{c}\text { Discharge } \\
(\mathrm{cfs})\end{array}$ & $\begin{array}{r}\text { Water } \\
\text { year }\end{array}$ & Date & $\begin{array}{c}\text { Gage } \\
\text { height } \\
\text { (feet) }\end{array}$ & $\begin{array}{c}\text { Di scharge } \\
\text { (cfs) }\end{array}$ \\
\hline 1958 & $\begin{array}{l}\text { July 28, } 1958 \\
\text { Sept.11, } 1958\end{array}$ & $\begin{array}{l}1.30 \\
2.64\end{array}$ & $\begin{array}{l}152 \\
550\end{array}$ & & July 14, 1960 & 0.84 & 78 \\
\hline 1959 & Aug. 5, 1959 & .70 & 58 & $\begin{array}{l}1961 \\
1962 \\
1963\end{array}$ & $\begin{array}{l}\text { Sept. 8, } 1961 \\
\text { Sept. 2, } 1962 \\
\text { Sept. 5, } 1962 \\
\text { Aug. 18, } 1963 \\
\end{array}$ & $\begin{array}{r}2.18 \\
1.93 \\
1.42 \\
.66 \\
\end{array}$ & $\begin{array}{r}374 \\
299 \\
175 \\
53 \\
\end{array}$ \\
\hline
\end{tabular}




\section{8-4245. Madera Canyon near Toyahvale, Tex.(24)}

Location.--Lat $30^{\circ} 52^{\prime}$, long $103^{\circ} 58^{\prime}$, In Jeff Davis County, 11 miles upstream from Aguja Canyon and 12 miles southwest of Toyahvale, Reeves County.

Dralnage area. $--53.8 \mathrm{sq} \mathrm{ml}$.

Gage.--Nonrecording prior to Dec. 16, 1932; recording thereafter. Altitude of gage is $4,200 \mathrm{ft}$ (from topographic map).

Stage-discharge relation.--Defined by current-meter measurements below $200 \mathrm{cfs}$ and by slope-area measurement at $3,700 \mathrm{cfs}$.

Bankfuli stage. $--10 \mathrm{ft}$.

Remarks.--Base for partial-duration series, 165 cfs.

Peak stages and discharges

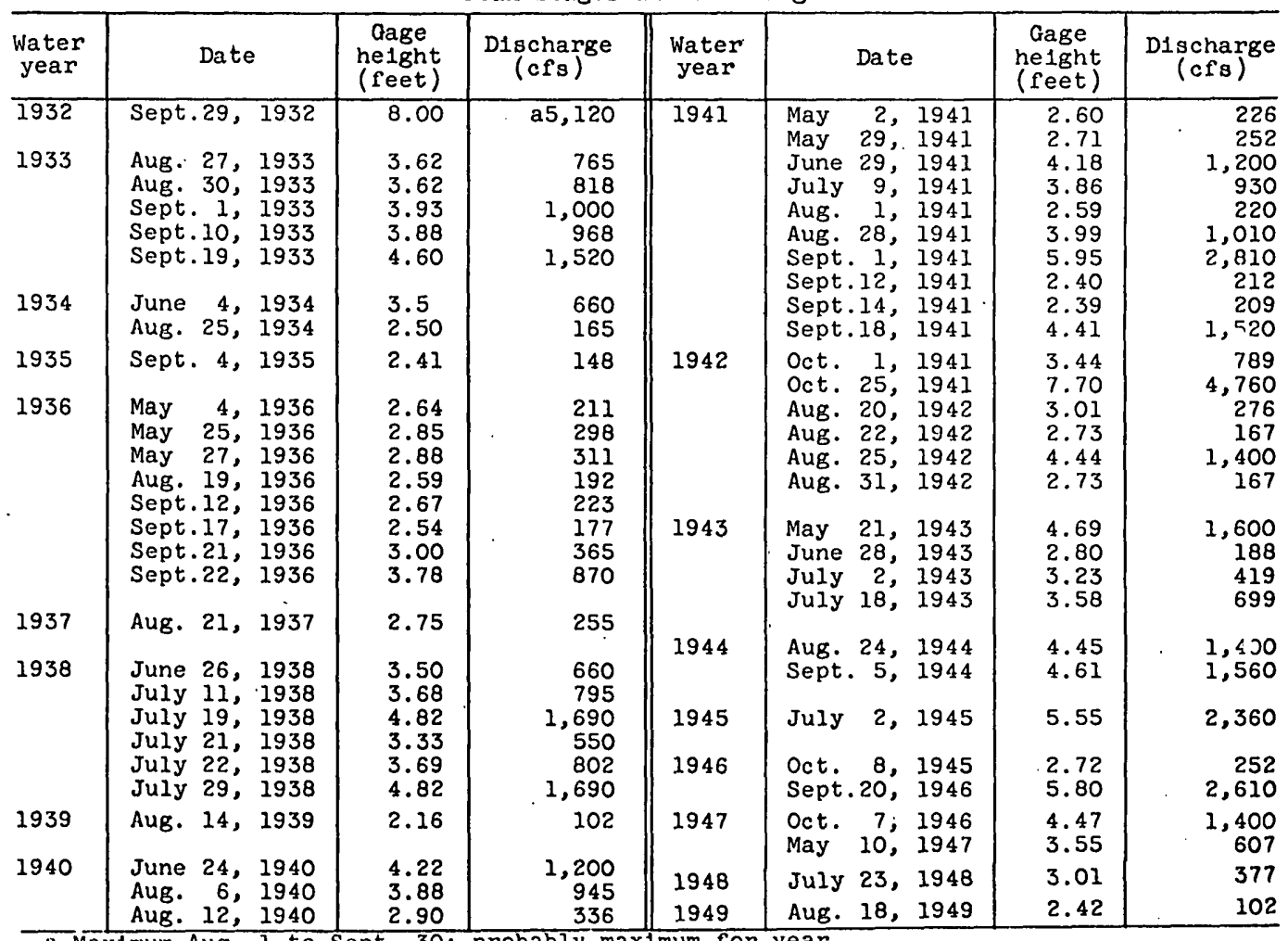

n Maxlmuin Aug. 1 to Sept. 30; probably maximum for year.

8-4515. Cienegas Creek near Del Rio, Tex. (22)

Location.--Lat $29^{\circ} 21^{\prime}$, long $100^{\circ} 57^{\prime}, 900 \mathrm{ft}$ upstream from mouth, $1 \frac{1}{2}$ miles upstream from Del Rio gaging station on the Rio Grande, and 3 miles northwest of Del Rio, Val Verde County.

Drainage area.--18 sq $\mathrm{mi}$.

Gage.--Recording. Datum unknown.

Remarks.--Records furnished by International Boundary and Water Commission. Only annual peaks are shown.

Peak stages and discharges

\begin{tabular}{c|c|c|c||c|c|c|c}
\hline $\begin{array}{c}\text { Water } \\
\text { year }\end{array}$ & Date & $\begin{array}{c}\text { Gage } \\
\text { height } \\
\text { (feet) }\end{array}$ & $\begin{array}{c}\text { Discharge } \\
\text { (cfs) }\end{array}$ & $\begin{array}{c}\text { Water } \\
\text { year }\end{array}$ & $\begin{array}{c}\text { Gage } \\
\text { height } \\
\text { (feet) }\end{array}$ & $\begin{array}{c}\text { Discharge } \\
\text { (cfs) }\end{array}$ \\
\hline 1931 & - & - & $(a)$ & 1934 & Sept. 3, 1934 & - \\
1932 & - & - & $(a)$ & 1935 & June 14, 1935 & 24.40 \\
1933 & - & - & (a) & 11,300 & 2,510 \\
\hline
\end{tabular}

a Not determined. 


\section{RIO GRANDE BASIN}

8-4530. San Felipe Creek near Del R1o, Tex. (22)

Location.--Lat $29^{\circ} 20^{\prime}$, long $100^{\circ} 53^{\prime}$, at S1los farm road bridge, 1.75 miles south of Del R10, Val Verde County, and 2 miles upstream from the mouth which is 1.6 miles downstream from International Bridge.

Drainage area. $--46 \mathrm{sq} \mathrm{m}$, all in the United States.

Gage.--Recording. At site $100 \mathrm{ft}$ downstream at datum $2.38 \mathrm{ft}$.lower prior to Jan. 1, 1956. Datum of gage is $877.43 \mathrm{ft}$ above mean sea level, U.S. Coast and Geodetic Survey datum.

Remarks.--Backwater reaches this station when the R10 Grande near Del Rio reaches a stage of $15 \mathrm{ft}$ or about $60,000 \mathrm{cfs}$. Records furnished by International Boundary and Water Commission. Only annual peaks are shown.

Peak stages and discharges

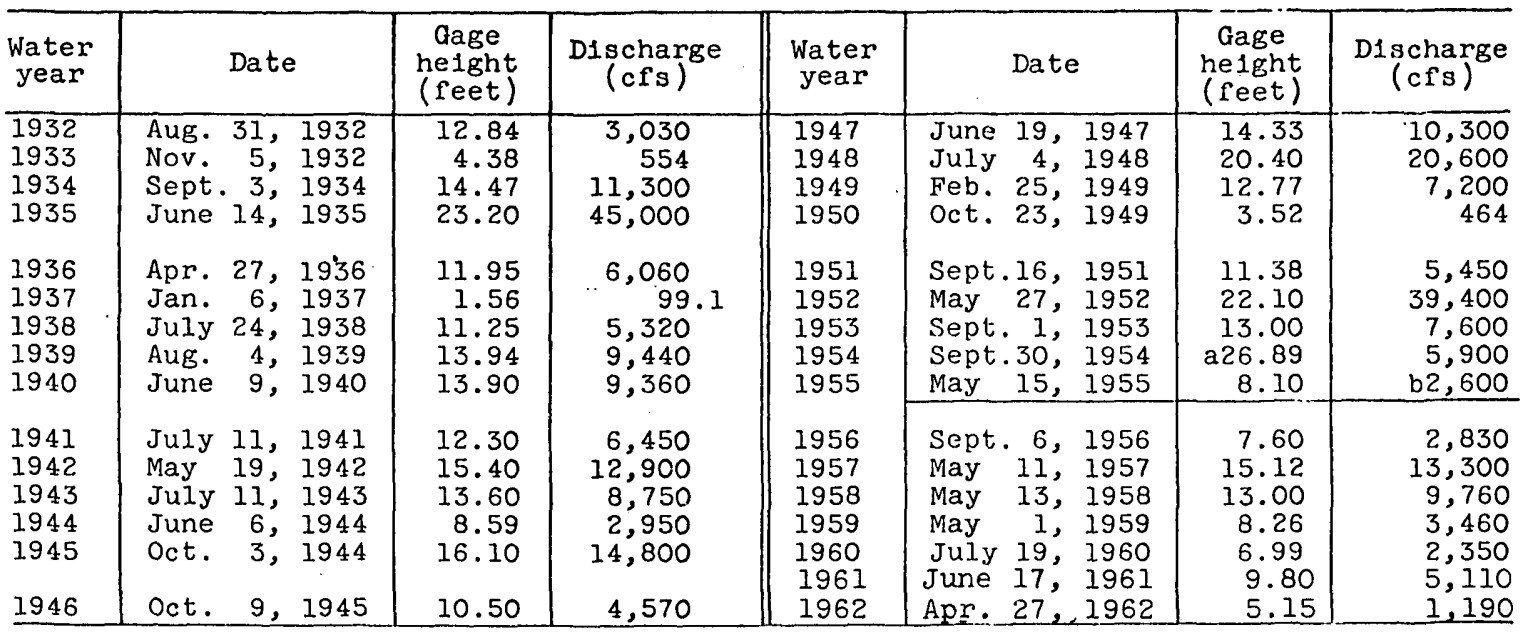

a Occurred on June 28, 1954; backwater from the R10 Grande.

b Maximum peak discharge; maximum discharge during year, 5,260 cfs on 0ct. 1, 1954, stage falling. 